\title{
A Determination of Air-Sea Gas Exchange and Upper Ocean Biological Production From Five Noble Gases and Tritiugenic Helium-3
}

\author{
by \\ Rachel H. R. Stanley \\ B.S. Massachusetts Institute of Technology, 2000 \\ Submitted in partial fulfillment of the requirements for the degree of Doctor of Philosophy \\ at the \\ MASSACHUSETTS INSTITUTE OF TECHNOLOGY \\ and the \\ WOODS HOLE OCEANOGRAPHIC INSTITUTION
}

September, 2007

(C) Rachel H.R. Stanley, MMVII. All rights reserved.

The author hereby grants to MIT and WHOI permission to reproduce paper and electronic copies of this thesis in whole or in part and to distribute them publicly.

Signature of Author

Joint Program in Chemicateceanography

Massachusetts Institute of Technology and Woods Holø Oceanographic Institution

August 10, 2007

Certified by

Dr. William J. Jenkins

Senior Scientist

Thesis Supervisor

Accepted by

Dr. Timothy I. Eglinton

Chair, Joint Committee for Chemical Oceanography

Senior Scientist

Woods Hole Oceanographic Institution 


\title{
A Determination of Air-Sea Gas Exchange and Upper Ocean Biological Production from \\ Five Noble Gases and Tritiugenic Helium-3
}

by

\author{
Rachel H. R. Stanley \\ Submitted to the Department of Marine Chemistry and Geochemistry, \\ Massachusetts Institute of Technology-Woods Hole Oceanographic Institution, \\ Joint Program in Chemical Oceanography \\ on August 10, 2007, in partial fulfillment of the \\ requirements for the degree of \\ Doctor of Philosophy
}

\begin{abstract}
The five noble gases (helium, neon, argon, krypton, and xenon) are biologically and chemically inert, making them ideal oceanographic tracers. Additionally, the noble gases have a wide range of solubilities and molecular diffusivities, and thus respond differently to physical forcing. Tritium, an isotope of hydrogen, is useful in tandem with its daughter helium-3 as a tracer for water mass ages. In this thesis, a fourteen month time-series of the five noble gases, helium-3 and tritium was measured at the Bermuda Atlantic Time-series Study (BATS) site. The time-series of five noble gases was used to develop a parameterization of air-sea gas exchange for oligotrophic waters and wind speeds between 0 and $13 \mathrm{~m} \mathrm{~s}^{-1}$ that explicitly includes bubble processes and that constrains diffusive gas exchange to $\pm 6 \%$ and complete and partial air injection processes to $\pm 15 \%$. Additionally, the parameterization is based on weeks to seasonal time scales, matching the time scales of many relevant biogeochemical cycles. The time-series of helium isotopes, tritium, argon, and oxygen was used to constrain upper ocean biological production. Specifically, the helium flux gauge technique was used to estimate new production, apparent oxygen utilization rates were used to quantify export production, and euphotic zone seasonal cycles of oxygen and argon were used to determine net community production. The concurrent use of these three methods allows examination of the relationship between the types of production and begins to address a number of apparent inconsistencies in the elemental budgets of carbon, oxygen, and nitrogen.
\end{abstract}

Thesis Supervisor: William Jenkins

Title: Senior Scientist, Woods Hole Oceanographic Institution 


\section{Acknowledgments}

I received funding towards my graduate research from the Department of Defense (NDSEG fellowship), the National Science Foundation (OCE-0221247), and the Scurlock Fund for research.

I would like to give a hundred thank yous to many people who have helped and supported me throughout my time here. First, I feel very lucky for having the opportunity of working with Bill Jenkins. Bill is an amazing advisor, a brilliant scientist and a genuinely wonderful person. Bill's advice, ideas, optimism, and good sense of humor have made the last six years so pleasurable. I sincerely thank my committee members Scott Doney, Jim Ledwell, and Paola Rizzoli. Scott spent an enormous amount of time with me discussing all the scientific questions raised in this study and teaching me how to poke and prod my model. I thank Jim for his thoughtful discussions on air-sea gas exchange and for his encouragement. Paola always made committee meetings a delight and asked very informative questions. Thanks also to Ed Boyle, my thesis defense chair. I am very grateful that Ed read my thesis and came to WHOI to chair my defense.

This work would not have been possible without Dempsey Lott. I am so thankful for Dempsey's expertise in building the mass spectrometer, and also for his patience in teaching me so much, for the care he has lavished on me, for his friendship, and for his very kind way of making me feel that eventually we would solve all our problems. I also thank past and present members of the Helium Isotope Lab for wonderful times and lots of help: Josh Curtice, Kevin Cahill, Burkard Baschek, Carolyn Walker, Zoe Howard-Bond, and Angela Landolfi. I am very grateful for the support and encouragement that Mark Kurz and Dave Glover have given to me throughout all of graduate school. I thank DeDe Toole and Naomi Levine for their friendship and support and also for the use of their computers for running my models.

I thank the WHOI Academic Programs Office for taking such good care of me and of all of us graduate students. It was always lovely to stop by the office and receive such a warm welcome. The administrative staff of the Marine Chemistry and Geochemistry department have been very helpful to me. I especially thank Donna and Sheila for helping me with forms and posters and room reservations and for always giving a smile and a warm word as well as the necessary signature or computer work.

I am grateful for the technical skill and dedication of Paul Keith, who not only welded many of the parts of the mass spectrometer so expertly, but who did it so kindly and quickly that it was always a pleasure to visit the welding shop. I also thank Charlie Clemshaw and Rich Zuks for their skill and hardwork.

My time here at WHOI has been enriched by the wonderful friendships I have made. The sometimes overwhelming task of writing a thesis was made possible thanks to the support and encouragement from many of my friends who were going through the same process themselves. I thank Clare, Anna, Jessica and Carlos for sharing these last few months of thesis writing frenzy. I thank Rose for her great support and encouragement - knowing I had a friend on the first floor made the evenings in my office pass quickly. I thank Nick for being one of my biggest and most special supporters throughout all of graduate school. And I thank Carolyn for sharing so much - an office, an advisor, a house, and so many wonderful times. I also thank my friends from before graduate school who have cheered me on for the last six years: Anna, Danielle, Laurie, Amanda, Liz, Karen, and Pascale.

I am grateful for all the support and encouragement that my family has lavished on me. I especially thank my father Gene for his care and advice and my brother Michael for his frequent pep talks. I sincerely thank my husband Dwight for giving me so much, advising me on science and math problems, encouraging me when nothing was working, and celebrating with me when things went well. The long days at work passed quickly because I knew that Dwight would be there for me when I finally went home.

In the interest of brevity, I give a final thank you to all my friends and colleagues. I do not have space here to thank each one individually but all have enriched my life for which I am sincerely grateful. 


\section{Dedication}

This thesis is dedicated to the memory of my mother, Idahlia Stanley. She was an amazing woman, a creative thinker, an inspired painter, and a wonderful mother. She taught me to ask questions and to give reasons. Her memory gives me courage and inspiration to tackle tough problems and comfort when the tackling is tough-going. 


\section{Contents}

1 Introduction $\quad 17$

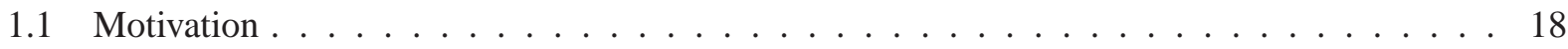

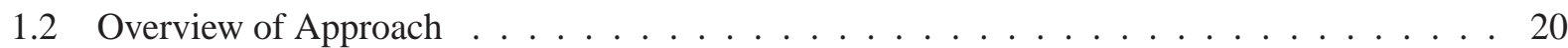

1.3 Chapter by Chapter Plan . . . . . . . . . . . . . . . . . . . . . 24

2 A Method for Measuring Five Noble Gases and Their Isotopic Ratios Using Stainless Steel Cryogenic Trapping and a Combination of Quadrupole and Magnetic Sector Mass Spectrom$\begin{array}{ll}\text { eters } & 27\end{array}$

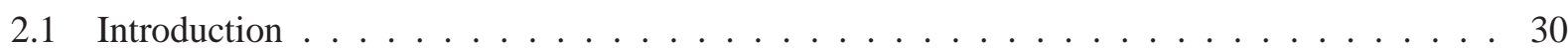

2.2 Methods . . . . . . . . . . . . . . . . . . . . . . . 31

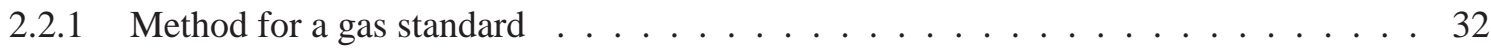

2.2.2 Additional Steps for a Water Sample . . . . . . . . . . . . . . . . . . . 38

2.2 .3 Details of QMS Analysis . . . . . . . . . . . . . . . . . . . . . 40

2.2.4 Details of Magnetic Sector Analysis . . . . . . . . . . . . . . . . . . . . . 40

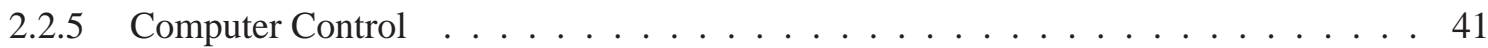

2.2 .6 Standardization . . . . . . . . . . . . . . . . . . . . . . 44

2.3 Analytical Performance and Reproducibility . . . . . . . . . . . . . . . . . . . 47

2.3.1 Performance of the QMS and Processing Line . . . . . . . . . . . . . . . . . . 47

2.3.2 Performance of the HIMS for He Isotopes . . . . . . . . . . . . . . . . . 59

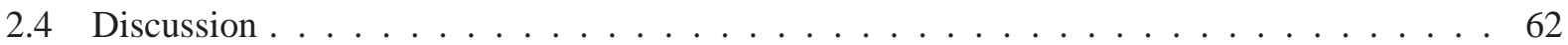

2.4.1 Separation of the Noble Gases . . . . . . . . . . . . . . . . . . . . 62

2.4 .2 Matrix Effects of $\mathrm{Ar}$ and $\mathrm{Xe}$ on $\mathrm{Kr} \ldots \ldots \ldots \ldots$. . . . . . . . . . . . 64

2.4 .3 Hydrogen . . . . . . . . . . . . . . . . . . . . . 66 
2.4 .4 Methane . . . . . . . . . . . . . . . . . . . . . . . . 68

2.4 .5 Error Analysis . . . . . . . . . . . . . . . . . . . . . . . 69

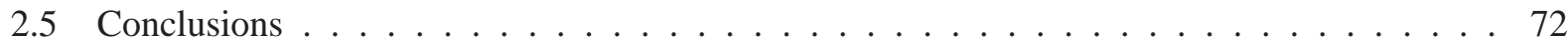

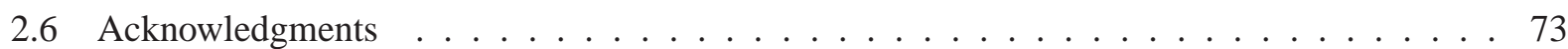

3 Quantifying Seasonal Air-Sea Gas Exchange Processes Using Noble Gas Time-Series: A Design Experiment $\quad 75$

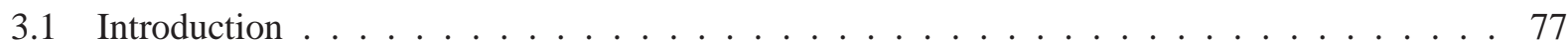

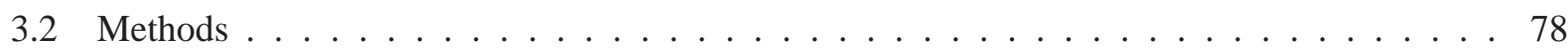

3.2.1 Description of the one-dimensional vertical upper ocean model: Physical parameters 78

3.2.2 Description of the model: Gas exchange parameters . . . . . . . . . . . . . 81

3.2.3 Linearization and inverse technique . . . . . . . . . . . . . . . 85

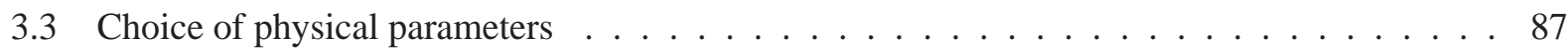

3.4 Sensitivity Study: Constraints on air-sea gas exchange parameters from the noble gases . . . 89

3.4.1 Model results: Noble gas behavior . . . . . . . . . . . . . . . . . . . . . . . 89

3.4.2 Quantifying the constraints . . . . . . . . . . . . . . . . . . . . . 94

3.5 Example application: Time-series of helium, neon, and argon . . . . . . . . . . . . . . 99

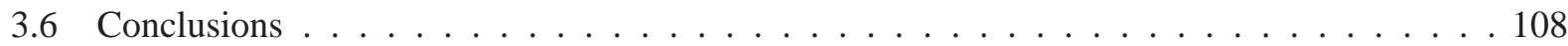

3.7 Acknowledgments . . . . . . . . . . . . . . . . . . . . . 110

4 Air-Sea Gas Exchange Parameters as Determined by a Time-Series of Five Noble Gases 113

4.1 Introduction . . . . . . . . . . . . . . . . . . . . . . . 115

4.2 Methods . . . . . . . . . . . . . . . . . . . . . . 117

$4.2 .1 \quad$ Data Collection . . . . . . . . . . . . . . . . . 117

4.2.2 Description of the one-dimensional vertical upper ocean model . . . . . . . . . . . . 121

4.2.3 Gas exchange parameterization used in the model . . . . . . . . . . . . . . . . 123

4.2 .4 Inverse Method . . . . . . . . . . . . . . . . . . . . . . . . . . 126

4.3 Results . . . . . . . . . . . . . . . . . . . . . . . 128

$4.3 .1 \quad$ Noble Gas Data . . . . . . . . . . . . . . . . . . . . . . . . 128

4.3 .2 Inverse Model Results . . . . . . . . . . . . . . . . . . . . . . 132

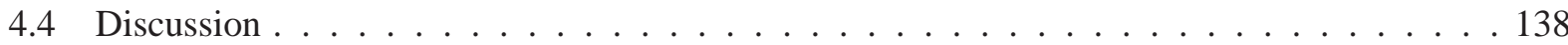


4.4 .1 The Base Case . . . . . . . . . . . . . . . . . . . . . . 138

4.4 .2 Controls on the Parameters . . . . . . . . . . . . . . . . . . . . 146

4.4 .3 Sensitivity of Parameters . . . . . . . . . . . . . . . . . . . . 147

4.5 Conclusions . . . . . . . . . . . . . . . . . . . . . . . 153

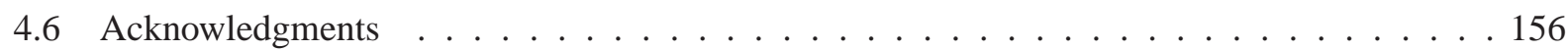

5 Estimates of Biological Production from a Time-Series of Noble Gases, Tritium, and Helium-3157

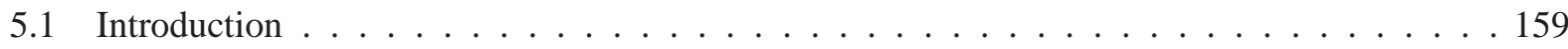

5.2 Methods . . . . . . . . . . . . . . . . . . . . . 162

5.2 .1 Data Collection . . . . . . . . . . . . . . . . . . . . 162

5.2 .2 He Flux Gauge Calculations . . . . . . . . . . . . . . . . . . . 165

5.2.3 Apparent Oxygen Utilization Rate Calculations . . . . . . . . . . . . . . . . . 167

5.2.4 Oxygen and Argon Time-Series Calculations . . . . . . . . . . . . . . . . 170

5.3 Results and Discussion . . . . . . . . . . . . . . . . . . . . . 173

5.3 .1 He Flux Gauge . . . . . . . . . . . . . . . . . . . . 173

5.3 .2 Apparent Oxygen Utilization Rates . . . . . . . . . . . . . . . . . . . . 184

5.3 .3 Oxygen and Argon Time-Series . . . . . . . . . . . . . . . . 193

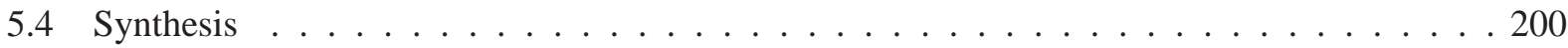

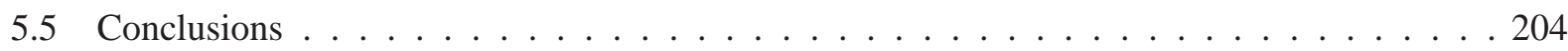

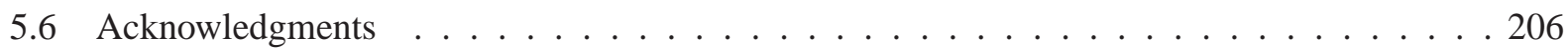

6 Conclusions and Future Directions $\quad 207$ 


\section{List of Figures}

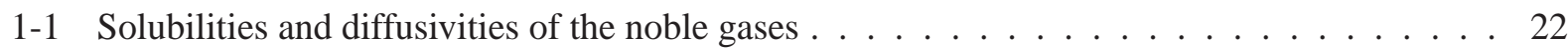

2-1 Schematic of the processing line and mass spectrometers. . . . . . . . . . . . . . 33

2-2 Temperature cycles of the stainless steel cryotrap . . . . . . . . . . . . . . . . 34

2-3 Release curves of the noble gases from the stainless steel cryotrap. . . . . . . . . . . . . 48

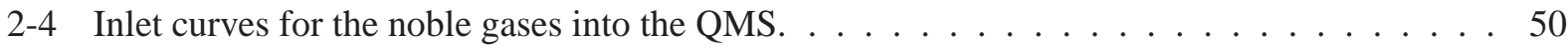

2-5 Peak shapes for the noble gases as measured by the QMS . . . . . . . . . . . . . . 53

2-6 Peak shapes for the noble gases at various cage voltages. . . . . . . . . . . . . . . . 54

2-7 Repeated QMS measurements on standards over several days. . . . . . . . . . . . . . . 57

2-8 Deviations of repeated QMS measurements of standards. . . . . . . . . . . . . 58

2-9 Peak shapes for He and HD as measured on the magnetic sector mass spectrometer. . . . . . 60

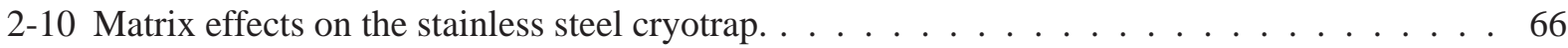

3-1 Comparison of temperature and salinity between model and BATS/Station S data $\ldots \ldots 8$

3-2 Surface saturation anomalies for the five noble gases and oxygen as predicted by the model . 90

3-3 Saturation anomalies in the top $300 \mathrm{~m}$ for the five noble gases as predicted by the model . . 91

3-4 Xe surface saturation anomaly for model runs with different gas exchange parameters . . . . 93

3-5 Linearization of observational metric as a function of model parameter . . . . . . . . . . 96

3-6 Time-Series of $\mathrm{He}, \mathrm{Ne}, \mathrm{Ar}$ data from $1989 \ldots \ldots$. . . . . . . . . . . . . 101

3-7 Modeled diffusive gas exchange, total air injection, and partial air injection fluxes for He,

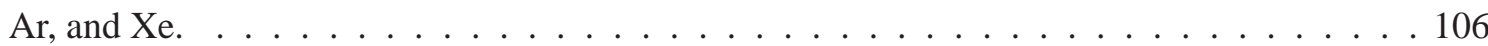

4-1 Upper ocean saturation anomalies of the five noble gases: data and model . . . . . . . . 129

4-2 Upper ocean concentrations of the five noble gases: data and model $\ldots \ldots \ldots$. . . . . . 130 
4-3 Surface saturation anomalies of the five noble gases $\ldots \ldots \ldots \ldots \ldots \ldots$

4-4 Vertical profiles of observed saturation anomalies for $\mathrm{He}$ and $\mathrm{Ne} \ldots \ldots$. . . . . . . 133

4-5 Vertical profiles of saturation anomalies for $\mathrm{Ar}, \mathrm{Kr}$ and $\mathrm{Xe} \ldots \ldots$. . . . . . . . . . . 134

4-6 Upper ocean temperature from BATS data and as predicted by model . . . . . . . . . . . . 140

4-7 Model-data differences of saturation anomalies and concentrations of the noble gases $\ldots 141$

4-8 Modeled fluxes of the noble gases . . . . . . . . . . . . . . . . . . . . . 143

4-9 Inventories of the noble gases in the upper $160 \mathrm{~m}$ in the base case model and from the data .145

4-10 Sensitivity of the noble gases to the proportion of complete to partial trapping. . . . . . . 148

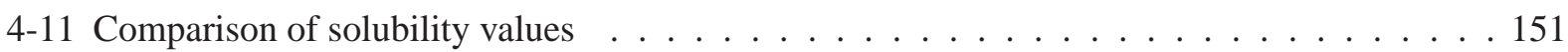

5-1 Box model for predictions for tritium, ${ }^{3} \mathrm{He}$, and T/He age as a function of replacement time . 169

$5-2$ Vertical profile of productivity in model f . . . . . . . . . . . . . . . 172

5-3 Seasonal modulation of productivity profile in model . . . . . . . . . . . . . . . . . 174

$5-4 \quad \delta^{3} \mathrm{He}$ isotope ratios, gas transfer velocity, and fluxes $\ldots \ldots \ldots \ldots$

5-5 Relationship between $\delta^{3} \mathrm{He}_{e x}$ and nitrate $\ldots \ldots \ldots \ldots \ldots \ldots \ldots$

$5-6$ Change in correlation of ${ }^{3} \mathrm{He}$ and $\mathrm{NO}_{3}^{-}$with time $\ldots \ldots \ldots \ldots \ldots \ldots$

5-7 Effect of air injection on equilibrium $\delta^{3} \mathrm{He} \ldots \ldots \ldots \ldots \ldots \ldots \ldots$

$5-8$ Time-series of tritium and excess ${ }^{3} \mathrm{He} \ldots \ldots \ldots \ldots$. . . . . . . . . . . . . . . . . . . . . . .

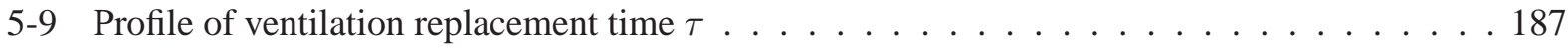

5-10 Profiles of apparent oxygen utilization rate . . . . . . . . . . . . . . . . . 189

5-11 Difference in saturation anomaly of $\mathrm{O}_{2}$ and $\mathrm{Ar}$ in model and data . . . . . . . . . . . . 194

5-12 Difference in saturation anomalies of $\mathrm{O}_{2}$ and $\mathrm{Ar}$ in the mixed layer . . . . . . . . . . . 197

5-13 Sensitivity study to seasonal amplitude of production . . . . . . . . . . . . . 198

6-1 Profile of the noble gases to $4200 \mathrm{~m}$ depth . . . . . . . . . . . . . . . . . 213 


\section{List of Tables}

2.1 Theoretical and experimentally determined amount of gas left behind in a sample bulb _ . . 39

2.2 Parameters used by the QMS to measure the five noble gases in a water sample or gas standard. 41

2.3 Measurement details for $\mathrm{He}, \mathrm{Ne}$ and $\mathrm{Ar} \ldots \ldots \ldots \ldots$. . . . . . . . . . . . . 42

2.4 Measurement details for $\mathrm{Kr}$ and $\mathrm{Xe} \ldots \ldots \ldots \ldots$. . . . . . . . . . . . . . . 43

2.5 Amount of gas in one aliquot of each type of gas standard . . . . . . . . . . . . . 46

2.6 Performance of the QMS for measuring the major isotopes of the five noble gases. . . . . . . 51

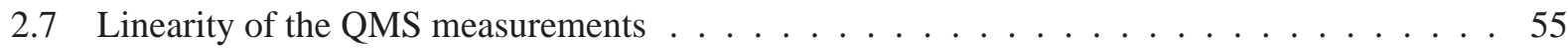

2.8 Performance of the QMS for distilled water samples . . . . . . . . . . . . . . . . . . . 59

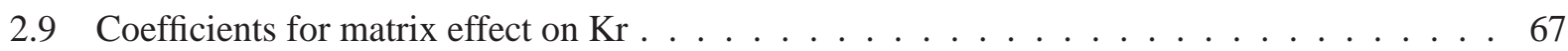

2.10 Sources of errors in the measurements of the noble gases. . . . . . . . . . . . . . . 70

3.1 Reference case values for tunable gas exchange parameters $\ldots \ldots \ldots$. . . . . . . . 86

3.2 Uncertainties on model parameters from the partial derivative method . . . . . . . . . . 9 95

3.3 Constrains on parameters from the full inverse method $\ldots \ldots \ldots$. . . . . . . . . 97

3.4 Observational metrics in example application . . . . . . . . . . . . . . . . . . 102

3.5 The slope matrix in the example application . . . . . . . . . . . . . . . 103

3.6 Model parameter values for the example application . . . . . . . . . . . . . . . . . . 104

3.7 Appendix: Definition of symbols and variables $\ldots \ldots \ldots \ldots \ldots \ldots$

4.1 Details of sample collection. . . . . . . . . . . . . . . . . . . . . 120

4.2 Contribution of bubbles entrained in samples during collection . . . . . . . . . . . . 121

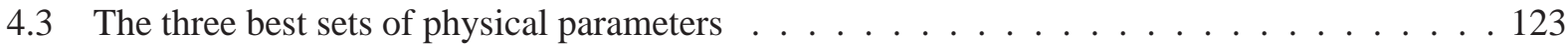

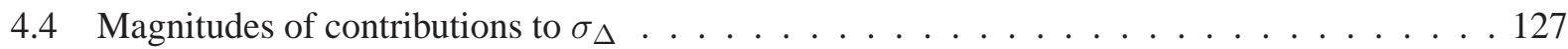

4.5 The air-sea gas exchange parameters as determined by the inverse modeling $\ldots \ldots$. . . . 135 
4.6 The corresponding fluxes as determined by the inverse modeling . . . . . . . . . 136

4.7 The air injection fluxes of the sum of the major gases as determined by the inverse modeling 137

4.8 The contributions of complete vs. partial trapping . . . . . . . . . . . . . . . 138

4.9 The sensitivity of the parameters to the exclusion of different gases . . . . . . . . . . . 147

5.1 Regression between $\delta^{3} \mathrm{He}_{e x}$ and $\mathrm{NO}_{3}^{-}$in the thermocline . . . . . . . . . 177

5.2 Estimates of biological production from this study . . . . . . . . . . . . . . . 179

5.3 Estimates of export production in different depth regions of the water column . . . . . . 190

5.4 Net community production as estimated from euphotic zone seasonal cycles of $\mathrm{O}_{2}$ and $\mathrm{Ar}$. . 195

5.5 Estimates of biological production from past research in the Sargasso Sea . . . . . . . . 202 
Chapter 1

Introduction 


\subsection{Motivation}

The ocean plays a key role in the biogeochemical cycle of many climatically relevant gases such as $\mathrm{CO}_{2}$, $\mathrm{CH}_{4}, \mathrm{~N}_{2} \mathrm{O}$, etc. These gases are at historically unprecedented levels in today's atmosphere (e.g. Keeling et al., 1996; Petit et al., 1999; Fluckiger et al., 1999; Spahni et al., 2005) and thus the exchange of gas between the atmosphere and the ocean is of particular importance. The ocean is a significant sink for anthropogenic $\mathrm{CO}_{2}$ (Siegenthaler and Sarmiento, 1993; Cox et al., 2000) but exactly how $\mathrm{CO}_{2}$ is sequestered and how this amount will change with time is not well understood. For example, recent evidence suggests that the Southern ocean sink of $\mathrm{CO}_{2}$ is becoming saturated (Le Quéré et al., 2007). Other gases than $\mathrm{CO}_{2}$ also have potentially varying sources and sinks in the ocean. The primary natural sources of $\mathrm{N}_{2} \mathrm{O}$ are emission from ocean upwelling regions and from tropical soils. Since the magnitude and location of denitrification may change with climate, the oceanic source of $\mathrm{N}_{2} \mathrm{O}$ may change as well. Another climatically relevant gas, DMS, is produced in the ocean and diffuses into the atmosphere, where through the CLAW hypothesis it may participate in important climate feedbacks (Charlson et al., 1987).

All gases in the atmosphere and ocean cross the air-sea boundary and thus are affected by air-sea gas exchange. The challenge is that air-sea gas exchange is a complicated, dynamical physical problem which is very difficult to measure directly. Thus bulk parameterizations of air-sea gas exchange flux have been developed to allow researchers to calculate the net result of air-sea gas exchange for a given gas in a given condition (Liss and Merlivat, 1986; Wanninkhof, 1992; Wanninkhof and McGillis, 1999; Nightingale et al., 2000). Recent studies suggest that these earlier estimates may be too large by approximately $20 \%$ to $30 \%$ (Ho et al., 2006; Sweeney et al., 2007). These air-sea flux relationships are important for global climate change models as well as for flux calculations. For example, bulk parameterizations have been used in conjunction with maps of surface seawater $\mathrm{pCO}_{2}$ to infer air-sea $\mathrm{CO}_{2}$ fluxes for different regions of the ocean (Takahashi et al., 1997). Additionally, quantification of air-sea gas exchange fluxes is necessary for biogeochemical research that uses gases as tracers to investigate key problems. For example, $\mathrm{O}_{2} / \mathrm{N}_{2}$ ratios in the atmosphere can be used to infer partitioning of $\mathrm{CO}_{2}$ between land and ocean sinks (e.g. Keeling et al., 1993; McKinley et al., 2003; Bender et al., 2005) and $\mathrm{O}_{2}$ measurements in the ocean can be used to infer net community production (e.g. Jenkins and Goldman, 1985; Craig and Hayward, 1987; Emerson, 1987). One sign of the ubiquity of use of these air-sea gas exchange parameterizations is that the most commonly used 
parameterization by Wanninkhof (1992) has been cited 890 times.

Existing parameterizations, however, have uncertainties of 25 to $50 \%$, leading to uncertainties that propagate through all studies using these parameterizations. Depending on the parameterization used, global and regional fluxes can differ by up to 100\% (Fangohr and Woolf, 2007). Additionally existing parameterizations are based on either short time-scales of hours to days or on long time-scales such as decades. The shortest time scales are assessed by micrometeorological techniques (Wanninkhof and McGillis, 1999) which have time scales of hours. Radon deficit calculations (Peng et al., 1979) and deliberate dual release experiments (Watson et al., 1991) allow prediction of gas exchange paramterizations on time scales of several days to two weeks. In line with the short time scales, these estimates are necessarily local in scope. At the other extreme, gas exchange parameterizations estimated from natural or bomb radiocarbon budget have decadal or longer time scales (Broecker and Peng, 1974; Wanninkhof, 1992; Sweeney et al., 2007). Such estimates are by nature global in scope. Yet it is the intermediate time scale of weeks to seasonal that matches the time scale of many biogeochemical processes in the ocean.

Moreover, most existing parameterizations do not explicitly treat bubbles. Air injection (the flux due to bubbles) is a complicated problem of bubble dynamics (e.g. Memery and Merlivat, 1985; Woolf and Thorpe, 1991; Woolf, 1993; Keeling, 1993; Woolf et al., 2007). The air injection flux can be significant, especially for less soluble gases such as $\mathrm{O}_{2}$ and He. Finally, the techniques that determine air-sea gas exchange parameters from direct empirical data, such as purposeful release experiments, are logistically difficult and have only been applied in limited areas of the ocean. The major goal of this thesis is therefore to determine an air-sea gas exchange parameterization with uncertainties of only 10 to $20 \%$ that is based on direct, emprical data, that explicitly includes bubbles, and that is based on weeks to seasonal time-scales.

Another reason for studying gases in the ocean is that gases can be used to constrain upper ocean biogeochemical cycles. Understanding the carbon cycle is key for climate research since $\mathrm{CO}_{2}$ is an important greenhouse gas. As mentioned above, $\mathrm{CO}_{2}$ enters the ocean through air-sea gas exchange. Marine organisms then fix approximately $50 \mathrm{Pg}$ of carbon per year (Field et al., 1998). Some of this organic matter is remineralized in the surface of the ocean and thus has no net effect on $\mathrm{CO}_{2}$ concentrations. Some of it, however, is exported into deep water and separated from the atmosphere on time scales of hundreds of years.

The importance of biological production in the ocean has long been known. Standard techniques for 
measuring biological production include bottle experiments in which radiotracers are added to bottles of seawater which are then incubated and analyzed. While such experiments are useful, they have limitations due to so-called "bottle effects" produced by confining production to a single bottle, eliminating grazers, trace metal contamination from the bottles, etc. Additionally, bottle experiments offer only a snap-shot of production at one particular place and time. Sediment traps quantify export production and offer the advantage of direct collection of sinking material. However, hydrodynamic biases and swimmers make sediment trap data difficult to interpret (Gardner, 2000). Newly designed neutrally-buoyant sediment traps (Buesseler et al., 2000; Valdes and Price, 2000; Stanley et al., 2004; Buesseler et al., 2007) avoid some of these problems but nonetheless sediment traps, as well as bottle experiments, may miss episodic events (such as production stimulated by eddies) which may constitute a large fraction of production (McGillicuddy et al., 2007).

Geochemical tracers complement the traditional approaches to estimating production because they characterize the integrated behavior of systems over broad spatial and temporal scales. A variety of geochemical tracers have been used to quantify three types of production - net community production (e.g. Jenkins and Goldman, 1985; Craig and Hayward, 1987; Spitzer and Jenkins, 1989; Gruber et al., 1998), export production (e.g. Jenkins, 1980; Sarmiento et al., 1990), and new production (Jenkins, 1988b; Jenkins and Doney, 2003). Net community production is defined as primary production minus heterotrophic respiration. New production is defined as the production stemming from input of new nutrients into the euphotic zone (Dugdale and Goering, 1967). Over sufficiently long temporal and spatial scales, the three types of production should be equal (Eppley and Peterson, 1979). In this study, I use geochemical tracers to measure all three types of production at the same location and at the same time for an in-depth analysis of the carbon cycle in a subtropical oligotrophic gyre. By using all these methods concurrently, I examine the relationship between the types of production and begin to address a number of apparent inconsistencies in the elemental budgets of carbon, oxygen, and nitrogen.

\subsection{Overview of Approach}

The two central objectives of my thesis are: 
1. To provide a quantitatively more accurate parameterization of air-sea gas exchange rates that explicitly includes bubble-mediated processes on biogeochemically relevant time scales

2. To use three tracer subsystems in order to concurrently quantify new, net community, and export production in an oligotrophic subtropical gyre.

In order to achieve these objectives, I have collected a monthly time-series of five noble gases $(\mathrm{He}, \mathrm{Ne}$, $\mathrm{Ar}, \mathrm{Kr}$, and $\mathrm{Xe}$ ), helium isotopes and tritium at the Bermuda Atlantic Time-series Study (BATS) site. I then combine this data with a one dimensional, vertical, modified Price-Weller-Pinkel (PWP) model (Price et al., 1986; Spitzer and Jenkins, 1989) in order to separate and quantify gas exchange parameters. By combining the noble gas and tritium data with biogeochemically active constituents, such as $\mathrm{NO}_{3}^{-}$and $\mathrm{O}_{2}, \mathrm{I}$ also constrain biological production.

Noble gases are ideal tracers because they are biologically and chemically inert and thus respond solely to physical forcing. Additionally, there are five of them with a range of solubilities and molecular diffusivities (Figure 1-1). The diffusivities differ by a factor of five with helium being the most diffusive (Jähne et al., 1987). The solubilities differ by a factor of ten, with xenon being the most soluble (Wood and Caputi, 1966; Weiss, 1971; Weiss and Kyser, 1978; Hamme and Emerson, 2004b). Additionally, the solubilities of the heavier noble gases have a stronger dependence on temperature. This broad range in physicochemical characteristics leads to differing response to physical forcing. Thus measurements of multiple noble gases made concurrently allow us to diagnose and quantify physical processes.

Helium $(\mathrm{He})$ and neon $(\mathrm{Ne})$, the lightest gases, are useful because they are insoluble with relatively little temperature dependence and therefore are sensitive to air injection processes. Krypton (Kr) and Xenon (Xe), the heaviest gases, are useful because they have a strong temperature dependence to solubility and thus respond to thermal forcing. Argon (Ar) is intermediate in behavior and has a solubility and diffusivity very similar to that of molecular oxygen. Thus, it is especially important because it can serve as an abiotic analogue to $\mathrm{O}_{2}$. Oxygen signatures in the ocean are a result of physics and biology. Argon mimics the physics and thus the difference between $\mathrm{O}_{2}$ and $\mathrm{Ar}$ can be a tracer for biological productivity (Craig and Hayward, 1987; Emerson, 1987; Spitzer and Jenkins, 1989). In practice, however, the story is more complicated since $\mathrm{Ar}$ and $\mathrm{O}_{2}$ have different gradients and distributions. In the aphotic zone, $\mathrm{O}_{2}$ is consumed through remineralization, resulting in an $\mathrm{O}_{2}$ debt that is mixed upward. Argon in contrast, has a relatively 
Figure 1-1: (a) Molecular diffusivities of the noble gases and oxygen as a function of temperature, as calculated from the diffusivity values of Jähne et al. (1987). Helium, the lightest noble gas, is more diffusive by a factor of five than Xe, the heaviest noble gas. (b) Solubilities of the five noble gases and oxygen as a function of temperature. The solubilities vary by an order of magnitude, with Xe being the most soluble and having the strongest temperature dependence. Solubility values for $\mathrm{He}$ are from a modified version of Weiss (1971), Ne and Ar solubilities are from Hamme and Emerson (2004b), Kr solubility is from Weiss and Kyser (1978) and Xe solubility is from Wood and Caputi (1966).
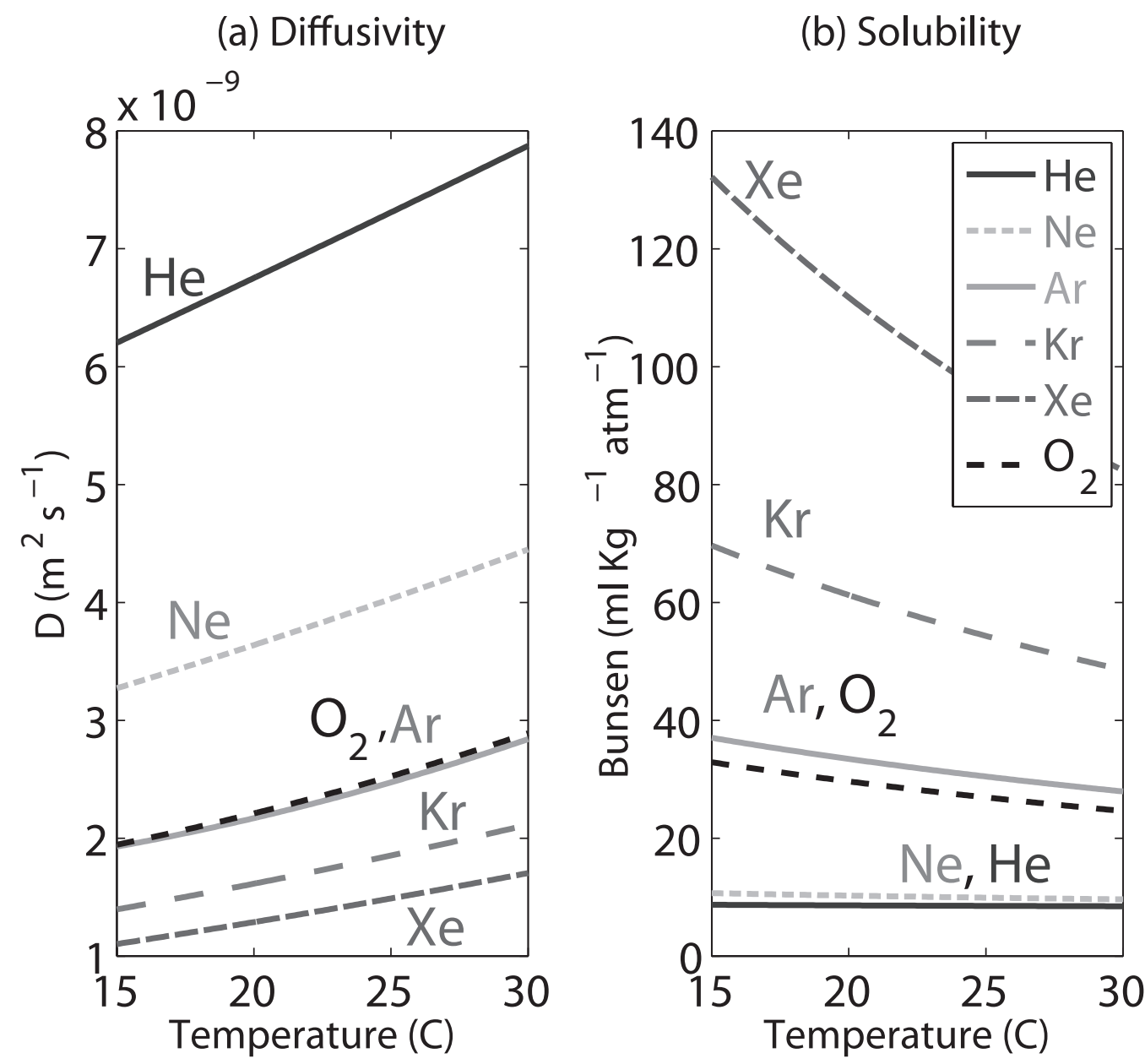
constant saturation anomaly with temperature. By using all the noble gases and a one dimensional model of upper ocean dynamics, I model the physical processes affecting $\mathrm{O}_{2}$ and by difference can estimate biological production.

In previous seawater studies, $\mathrm{Ar}$ has been used in conjunction with $\mathrm{O}_{2}$ to estimate biological production (Craig and Hayward, 1987; Spitzer and Jenkins, 1989). Time-series of He, Ne and Ar measurements in seawater have been used to investigate air-sea gas exchange (Spitzer and Jenkins, 1989) as have time-series of $\mathrm{Ne}, \mathrm{Ar}$, and $\mathrm{N}_{2}$ (Hamme and Emerson, 2006). Helium measurements in seawater have been used to investigate escape mechanisms for the exosphere (Bieri et al., 1967). Modeling studies suggest Ar can be used for quantifying diapycnal mixing (Henning et al., 2006; Ito and Deutsch, 2006). The solubility of $\mathrm{Kr}$ and Xe have a stronger thermal dependency than Ar and thus these heavier gases allow better constraints on air-sea gas exchange parameters (Stanley et al., 2006) and may be useful tracers of water mass formation processes (Hamme and Severinghaus, 2007).

As part of our time-series, I also measure ${ }^{3} \mathrm{He}$ and tritium. ${ }^{3} \mathrm{He}$ in the ocean has two sources - primordial ${ }^{3} \mathrm{He}$ from hydrothermal and volcanic activity and ${ }^{3} \mathrm{He}$ produced from in-situ tritium decay. In the upper ocean at BATS, the main source of ${ }^{3} \mathrm{He}$ is tritium decay (Jenkins, 1980). Tritium is naturally produced in small amounts from cosmogenic rays, but the natural inventory was dwarfed by input of tritium from the thermonuclear bomb tests in the 1950s and 1960s. Tritium was injected into the stratosphere where it formed HTO and then rained out into the ocean. Tritium decays to ${ }^{3} \mathrm{He}$ with a half-life of 12.31 years (MacMahon, 2006) and the combination of tritium and ${ }^{3} \mathrm{He}$ measurements can be used as a "clock" for dating subsurface water (Jenkins and Clarke, 1976). At the surface, the clock is zeroed as most of the ${ }^{3} \mathrm{He}$ is fluxed out due to gas exchange. As water is subducted and separated from the atmosphere, ${ }^{3} \mathrm{He}$ builds up from decay of tritium. In practice, mixing complicates this simple scenario but models can be used to calculate ventilation time scales from tritium and ${ }^{3} \mathrm{He}$ measurements (Jenkins, 1980; Doney and Jenkins, 1988).

By measuring a time-series of tritium, ${ }^{3} \mathrm{He}$, and the noble gases, I am able to resolve the seasonal cycle and build a detailed understanding of the dynamic system. The BATS site is an ideal place for this work for several reasons. First, a time-series of many biogeochemical relevant parameters (but not the noble gases) have been measured at BATS since 1988 (Michaels and Knap, 1996) and before that at Station S since 1954 (Schroeder and Stommel, 1969). Thus there is a wealth of historical data that can be used to constrain the 
model and to interpret the results. Second, the infrastructure was in place for sample collection. This not only meant that ship-time was accessible, but also key information such as $\mathrm{O}_{2}$ and nutrient measurements are made as part of the BATS program and ancillary data from the BATS program (such as plankton tows, sediment traps, bottle experiments, etc) may aid in interpretation of results. Third, there is a significant seasonal cycle at BATS, with a summer to winter temperature difference of approximately 10 degrees. Since the heavier noble gases respond most strongly to thermal forcing, a strong temperature cycle causes a supersaturation of the gases in summer, and thus a flux out of the mixed layer which can be parameterized. Fourth, BATS is located in an oligotrophic regime. Ninety percent by area of the oceans are oligotrophic and half of the global carbon export occurs in oligotrophic regimes. Thus a study in such conditions is relevant to much of the world's oceans. Finally, noble gas data at the BATS site is amenable to being modeled with a one-dimensional vertical model. The main factor controlling the distribution of the noble gases is the temperature history of the water. At BATS, the contours of net heat flux parallel the circulation (Worthington, 1976) and thus a one-dimensional model can reasonably be used for the noble gases .

I use a one-dimensional vertical Price-Weller-Pinkel (PWP) (Price et al., 1986) model that has been modified to include He, Ne, and Ar (Spitzer and Jenkins, 1989) and I extend it to the heavier noble gases. I force the model with six-hourly NCEP reanalysis heat flux (Kistler et al., 2001) and with QuikSCAT winds. Modeling is necessary to separate and quantify the physical processes affecting the noble gases. The advantage of a one-dimensional model is that it is simple and transparent. However, a one-dimensional model clearly does not account for horizontal processes and large scale circulation. For reasons described above, the model is sufficient for the noble gases. However, it is not appropriate for use with nutrients, tritiugenic ${ }^{3} \mathrm{He}$, and tritium because of large scale gyre circulation (Williams and Follows, 1998; Jenkins and Doney, 2003).

\subsection{Chapter by Chapter Plan}

Noble gases are ideal tracers but the heavier noble gases have not often been measured in seawater, perhaps because they are so difficult to measure. Recent improvements in mass spectrometers, particularly the development of a stainless steel cryogenic trap (Lott, 2001) have made such measurements possible. In Chapter 2 of this thesis, I describe a method I developed for measuring the five noble gases and their isotopes 
in seawater samples through mass spectrometry. Automated cryogenic traps are used to first sorb and then to separate the noble gases. Two mass spectrometers are attached to a single processing line, allowing measurements of both helium isotopes and noble gases on the same samples. The noble gases are measured statically by peak height manometry using a quadrupole mass spectrometer, equipped with a pulse-counting secondary electron multiplier. Helium isotopes are measured on a purposely built, branch-tube, statically operated magnetic sector mass spectrometer. Separating the noble gases in a reproducible way can be difficult due to matrix effects of one noble gas on another as well as affects from other gases in seawater (such as methane) and thus great effort was made to reduce and assess matrix effects. Additionally, the mass spectrometer and processing system are precisely standardized, and any effects of nonlinearity are assessed and accounted for. By measuring the noble gases through comparison to precisely known aliquots of air, I avoid using isotope dilution and thus can determine the isotopic ratios of the noble gases in the samples.

In Chapter 3, I perform a modeling sensitivity study in order to determine how well a time-series of five noble gases at BATS could constrain air-sea gas exchange parameters. I extend the PWP model for Kr and Xe and force the model with NCEP reanalysis winds and heat fluxes (Kistler et al., 2001). Ensemble runs are used to optimize tunable physical parameters in order to emulate the temperature, salinity, and mixed layer observations at BATS. I then perform sensitivity studies to characterize the response of the noble gas saturation anomalies to air-sea gas exchange parameters. I use a linear inverse technique (singular value decomposition) in order to determine the constraints offered by a hypothetical time-series of all five gases. As a limited demonstration of the approach, I use a dataset of a time-series of three noble gases $(\mathrm{He}, \mathrm{Ne}$, and Ar) collected between 1985 and 1988 in the Sargasso sea (Spitzer, 1989) in order to calculate preliminary estimates of air-sea gas exchange parameters.

In Chapter 4, I present a 14 month time-series of five noble gases collected between July 2004 and August 2005 at 22 depths in the upper $400 \mathrm{~m}$ of the ocean. I combine the noble gas data (measured according to the method in Chapter 2) with inverse modeling (as in Chapter 3 but with QuikSCAT winds) in order to develop an air-sea gas exchange parameterization that explicitly includes air injection processes, that has uncertainties of $\pm 6 \%$ for diffusive gas exchange and $\pm 15 \%$ for air injection over the range of wind speeds encountered in the time-series $\left(0<u_{10}<13 \mathrm{~m} \mathrm{~s}^{-1}\right)$, and that provides an estimate on a relevant and unique time scale. I use a nonlinear constrained optimization inverse method and explore the sensitivity of the 
parameters to uncertainties in the solubility functions of the noble gases, to uncertainties in the physical parameters used in the model, to the structure of the cost function, and to uncertainties in the model's representations of the gases.

In Chapter 5, I combine the gas exchange parameterization developed in Chapter 4 with ${ }^{3} \mathrm{He}$, tritium, $\mathrm{O}_{2}$, and $\mathrm{NO}_{3}^{-}$measurements from the 14 month time-series in order to estimate new, net community, and export production. First, I combine ${ }^{3} \mathrm{He}$ measurements in the mixed layer with the gas exchange parameterization to calculate the ${ }^{3} \mathrm{He}$ flux out of the mixed layer. By correlating ${ }^{3} \mathrm{He}$ in the thermocline with $\mathrm{NO}_{3}^{-}$, I can then estimate the flux of $\mathrm{NO}_{3}^{-}$into the mixed layer and the new production flux as it is physically transported by upwelling of thermocline waters. I next use tritium and ${ }^{3} \mathrm{He}$ data from the upper ocean measurements as well as from two profiles that extend to $4200 \mathrm{~m}$ depth in order to calculate the ventilation age of the water. I combine apparent oxygen utilization with these ventilation ages to calculate apparent oxygen utilization rates (AOUR). The vertically integrated AOUR is a measure of export production. Finally, I use the euphotic zone seasonal cycles of $\mathrm{O}_{2}$ and Ar with the model developed in Chapters 2 and 3, in order to estimate net community production. This study is unique in that it measures all three types of production using geochemical techniques at the same location and at the same time. By comparing the estimates of the three types of production, I start to examine inconsistencies in the elemental cycling of $\mathrm{C}, \mathrm{O}$, and $\mathrm{N}$.

In summary, this thesis uses the five noble gases as tracers to improve our understanding of air-sea gas exchange processes and upper ocean biological production. The noble gases are used to develop an improved parameterization of air-sea gas exchange that explicitly includes bubbles and that can be applied to calculate the air-sea flux of any gas. The noble gases, tritiugenic ${ }^{3} \mathrm{He}$, and tritium are then used, in conjunction with this improved parameterization, to estimate three types of biological production in a subtropical oligotrophic gyre and to explore the relationship between new, net community, and export production and nutrient cycling. 
Chapter 2

A Method for Measuring Five Noble Gases and Their Isotopic Ratios Using Stainless Steel Cryogenic Trapping and a Combination of Quadrupole and Magnetic Sector Mass Spectrometers 


\begin{abstract}
A method is presented for precisely measuring all five noble gases and their isotopic ratios in water samples using multiple cryogenic traps in conjunction with quadrupole mass spectrometry and magnetic sector mass spectrometry. Multiple automated cryogenic traps, including a two-stage cryotrap used for removal of water vapor, an activated charcoal cryotrap used for helium separation, and a stainless steel cryotrap used for neon, argon, krypton and xenon separation, allow reproducible gas purification and separation. The precision (expressed as 1 standard deviation) of this method, determined by repeated measurements on gas standards, is $\pm 0.10 \%$ for $\mathrm{He}, \pm 0.14 \%$ for $\mathrm{Ne}, \pm 0.10 \%$ for $\mathrm{Ar}, \pm 0.14 \%$ for $\mathrm{Kr}$, and $\pm 0.17 \%$ for $\mathrm{Xe}$. The precision of this method for water samples, determined by measurement of duplicate pairs, is $\pm 1 \%$ for $\mathrm{He}, \pm 0.9 \%$ for $\mathrm{Ne}, \pm 0.3 \%$ for $\mathrm{Ar}, \pm 0.3 \%$ for $\mathrm{Kr}$, and $\pm 0.2 \%$ for Xe. Isotopic ratios of the noble gases are measured as well, with precisions of $\pm 0.19 \%$ for ${ }^{20} \mathrm{Ne} /{ }^{22} \mathrm{Ne}, \pm 0.20 \%$ for ${ }^{84} \mathrm{Kr} /{ }^{86} \mathrm{Kr}, \pm 0.25 \%$ for ${ }^{84} \mathrm{Kr} /{ }^{82} \mathrm{Kr}, \pm 0.09 \%$ for ${ }^{132} \mathrm{Xe} /{ }^{129} \mathrm{Xe}$ and $\pm 0.13 \%$ for ${ }^{132} \mathrm{Xe} /{ }^{136} \mathrm{Xe}$. An attached magnetic sector mass spectrometer measures ${ }^{3} \mathrm{He} /{ }^{4} \mathrm{He}$ with precisions of $\pm 0.1 \%$ for air standards and $\pm 0.14 \%$ for water samples.
\end{abstract}




\subsection{Introduction}

Noble gases are biologically and chemically inert and have a wide range of solubilities and diffusivities, making them useful environmental tracers. Noble gases have also been used extensively in groundwater studies to constrain paleotemperatures (Stute et al., 1992, 1995; Aeschbach-Hertig et al., 2000) and groundwater infiltration and recharge (Beyerle et al., 1999; Manning and Solomon, 2003; Zhou et al., 2005). Measurements of $\mathrm{Ar}$ and $\mathrm{Kr}$ isotopes in ice cores can lead to estimation of firn thickness and temperature (Craig and Wiens, 1996; Severinghaus et al., 2001, 2003). Noble gases in mantle-derived rocks have been used to infer properties of the early history of the earth (Honda et al., 1991; Hiyagon et al., 1992; Farley and Neroda, 1998). Noble gas isotopes produced by cosmic rays yield surface exposure ages for terrestrial rocks (Lal, 1991; Bierman, 1994; Schafer et al., 1999).

In seawater studies, noble gas measurements have been used to investigate air-sea gas exchange (Spitzer and Jenkins, 1989; Emerson et al., 1995; Hamme and Emerson, 2006), biological production (Jenkins and Goldman, 1985; Craig and Hayward, 1987), diapycnal mixing (Henning et al., 2006; Ito and Deutsch, 2006), and escape mechanisms for the exosphere (Bieri et al., 1967). Extending the measurements beyond the three noble gases $(\mathrm{He}, \mathrm{Ne}$, and $\mathrm{Ar})$ used in previous work allows one to constrain air-sea gas exchange parameters to levels significantly better than can be obtained when only three of the noble gases are used (Stanley et al., 2006). Given that $\mathrm{Kr}$ and $\mathrm{Xe}$ have a stronger thermal dependency than Ar, the supersaturation pattern of the heavier noble gases may provide probes for ocean mixing and water mass formation processes (Hamme and Severinghaus, 2007). Additionally, the isotopic ratios of the noble gases could be useful tracers.

Quadrupole mass spectrometry (QMS) and magnetic sector mass spectrometry have often been used to measure the noble gases in water samples. Recently, instruments have been developed that measure all five noble gases from a single sample (Poole et al., 1997; Beyerle et al., 2000; Kulongoski and Hilton, 2002; Sano and Takahata, 2005). The analysis is usually conducted by isotope dilution or by peak height comparison with an air standard. The noble gases are commonly chemically purified and then condensed onto a charcoal trap at liquid $\mathrm{N}_{2}$ temperatures ( $77 \mathrm{~K}$ ), on a charcoal trap at dry ice/acetone temperature (96K), or on a glass trap at liquid $\mathrm{He}(4 \mathrm{~K})$. Methods that measure all five gases from a single sample have precisions of $0.3 \%$ to $1.0 \%$ using magnetic sector instrument (Beyerle et al., 2000) and $0.4 \%$ to $1.6 \%$ using a QMS system (Sano and Takahata, 2005). Methods that only measure one of the noble gases may have better precisions. For 
example, an isotope dilution method for measuring only Ne routinely obtained precisions of $0.13 \%$ (Hamme and Emerson, 2004a).

We have developed an automated sample processing and measurement system for the determination of the complete set of noble gas concentrations and isotope ratios in water samples $(90 \mathrm{cc})$ at the $0.1-0.2 \%$ level. Active gases are removed from the sample and the remaining noble gases are captured cryogenically and then selectively released for measurement to a quadrupole or a magnetic sector mass spectrometer. We use a set of three cryogenic traps operated in tandem with a Pd catalyst and chemical getters and combined with a volume partitioning system in order to achieve a high degree of purity and separation between the individual noble gases, thereby reducing as much as possible the potential for interference between gas species. The water vapor cryotrap (WVC) is a dual flow through cryotrap with independently controlled temperatures allowing water vapor to be removed but the noble gases to pass unimpeded. The activated charcoal cryotrap (ACC) captures He and then releases an aliquot into the QMS and the remainder into a helium isotope magnetic sector mass spectromer (HIMS) for precise measurements of ${ }^{3} \mathrm{He} /{ }^{4} \mathrm{He}$ ratios. A "nude" stainless steel cryotrap (SSC) (Lott, 2001) captures Ne, Ar, Kr and Xe and then selectively releases the gases into the QMS. The QMS operates in a static, ion-counting mode which requires a lower partial pressure of gas within the QMS, less gain dependence on the electron multiplier, and a more linear response. Standardization of the system is accomplished using precisely known aliquots of atmospheric noble gases (determined by pressure, temperature, volume and relative humidity), and thus is dependent on knowledge of the abundance of these gases in air. However, since studies involving dissolved gases generally presume these values, uncertainties in these abundances cancel out in most calculations.

\subsection{Methods}

The sample processing and measurement system is shown in Fig. 2-1. Noble gases from a water sample or gas standard are sequentially drawn through the two-stage WVC to remove water vapor, and through a Pd catalyst and getters for chemical purification, and then onto two cryogenic traps. The AAC at $<10 \mathrm{~K}$ captures $\mathrm{He}$ and then at $40 \mathrm{~K}$ releases He into the HIMS. The SSC, also initially at $<10 \mathrm{~K}$, captures $\mathrm{Ne}$, Ar, $\mathrm{Kr}$, and $\mathrm{Xe}$ and then selectively warms and releases the noble gases (Fig. 2-2) into the statically operated QMS for measurement by peak height manometry. "Static" refers to the sample being let into the isolated 
QMS (i.e. no pumping during measurement) and then peak jumping is used to measure the count rates at each of the pre-determined masses. The QMS is a Hiden quadrupole mass spectrometer (P/N PCI 1000 1.2HAL/3F 1301-9 PIC type 570309), equipped with a pulse counting secondary electron multiplier (SEM) run at an emission of 20 to $40 \mu \mathrm{amps}$, an electron impact ion source and triple quadrupole ion optics. The HIMS, an improved system based on the "Clarke design", is a purposely constructed branch tube, statically operated, dual collector magnetic sector helium isotope mass spectrometer, radius of $25.4 \mathrm{~cm}$, equipped with a Faraday cup and a pulse counting SEM. The system, including the processing line, cryotraps, and mass spectrometers, is operated under computer program control to achieve a high degree of reproducibility and for continuous operation.

In order to avoid possible systematic biases caused by the interaction between gas species via ion collisions and preferential ionization, we use cryogenic techniques to separate the noble gases before they are inlet into the mass spectrometers. Thus each of the noble gases is measured sequentially from the same air standard or water sample. The cryogenic systems used here lead to three cryogenic processes: cryocondensation, cryosorption and cryotrapping. Cryocondensation refers to the condensation of gas on a truly inert surface and results in the partial pressure of the gas above the surface being a function only of its vapor pressure at the trap temperature. However, no surface is truly inert, and thus the nature of the surface of the trap influences the amount of gas released. An active surface, such as on the ACC, results in stronger cryosorption and thus releases the gases at higher temperatures than a stainless steel surface such as on the SSC. For example, $\mathrm{Ne}$ is released at $25 \mathrm{~K}$ on the $\mathrm{SSC}$ and at $80 \mathrm{~K}$ on the AAC.

Cryotrapping refers to one gas being trapped underneath another. Since Ar is three to five orders of magnitude more abundant than the other noble gases, Ar cryotraps the other gases and thus the SSC has to go through many temperature cycles to reproducibly and quantitatively separate and release all the noble gases. In this section, we first outline the process necessary for measuring all five noble gases in a single air standard. Then we discuss some additional particulars that are necessary for processing a water sample.

\subsubsection{Method for a gas standard}

The processing line is pumped by a diffusion pump, a turbo molecular pump, and three ion pumps (in different parts of the line - see Fig 2-1) to below $5 \times 10^{-8}$ torr before starting an analysis. An aliquot of an air 
Figure 2-1: Schematic of the processing line and mass spectrometers (HIMS and QMS) that comprise the analytical system. Red and green squares denote pneumatically actuated copper stem tip (vacuum-type) stainless steel UHV bellows valves (Nupro P/N 22-*BG-TW-CU-3C and SS-4BG-USI-VD-3C), with red squares representing the valves that are closed at the beginning of an analysis and green squares representing the valves that are open at the beginning of an analysis. Valve numbers are included for valves referenced in the text. White squares represent pneumatically operated crackers. Ovals represent aliquot volumes. All aliquots as well as the standard reservoir volumes and the $2 \mathrm{~L}$ expansion volume are in an aluminum box in order to keep them at an approximately constant temperature. Purple squares denote pressure gauges with "IG" representing ionization gauges (Granville-Phillips 330), "CV" representing convectron gauges (Granville-Phillips 316), and "MKS" representing a capacitance manometer (MKS baratron PR4000 controller with 10 torr type 626A absolute pressure transducer). " $1 \mathrm{~N}_{2}$ trap" refers to a trap chilled with liquid nitrogen to condense water vapor when initially pumping down the sample bulbs after attaching them to the automanifold - the sample bulbs are still sealed at that point. "Dual WVC" refers to a two-stage cryotrap (inlet and outlet sides) initially held at $160 \mathrm{~K}$ to trap water in the sample. "AAC" and "SSC" refer to the activated charcoal and the stainless steel cryotraps respectively. For more detail on the processing line, please refer to the text.

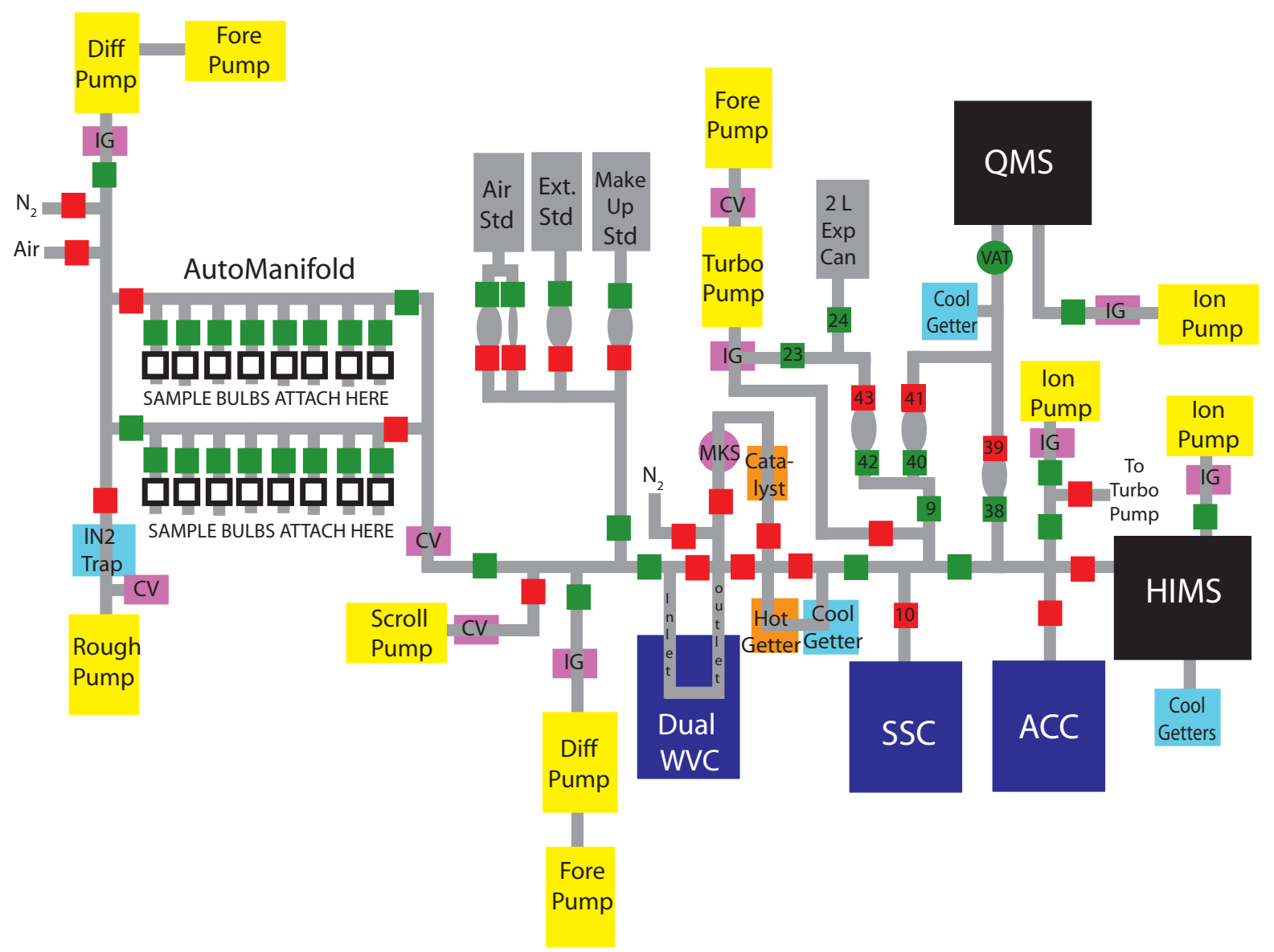


Figure 2-2: Temperature of the stainless steel cryotrap (SSC) as a function of time after analysis has commenced. The SSC undergoes a number of temperature cycles in order to reproducibly separate and release the noble gases. Gray arrows indicate where gases are released. Black arrows indicate where gases are pumped. Numbers indicate temperatures at various points in the temperature cycles.

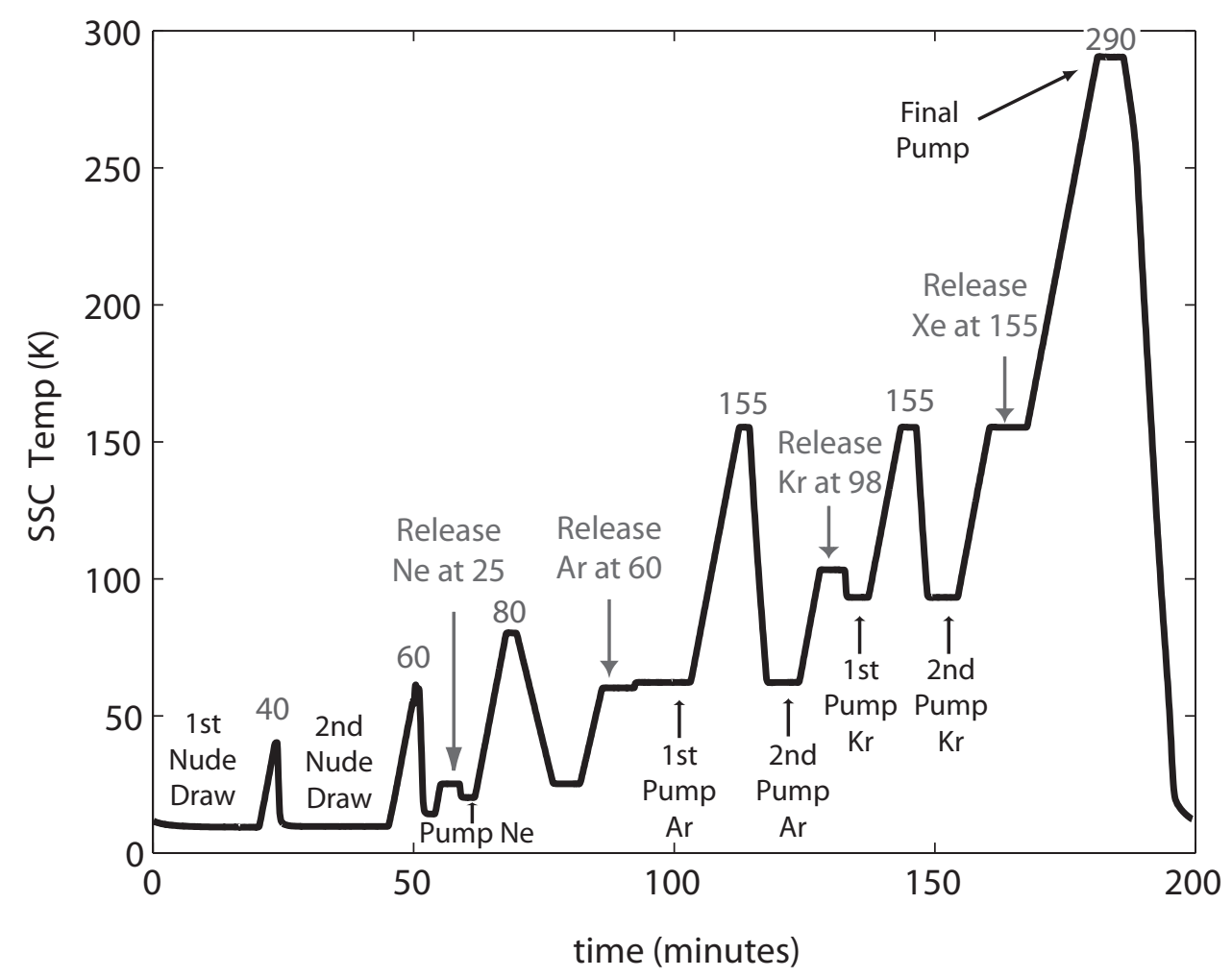


standard or a sample first passes through the WVC, a two-sided cryotrap held at $160 \mathrm{~K}$, in order to remove water vapor. Then it flows through a Pd catalyst (BASF catalyst $\mathrm{R} 02-20 / 37$ ) where the $\mathrm{CH}_{4}$ in the standard is oxidized to $\mathrm{CO}_{2}$ and $\mathrm{H}_{2} \mathrm{O}$. The pressure is recorded on a capacitance manometer (MKS baratron PR4000 controller with 10 torr type 626A absolute pressure transducer) in order to get a parametric determination of the total gas pressure. The gas then flows through a zirconium-vanadium-iron getter, composed of pellets of STS707 (available from SAES getters) in order to remove active gases. The first half of the getter is heated to $350^{\circ} \mathrm{C}$ to chemically remove $\mathrm{O}_{2}, \mathrm{~N}_{2}$, and $\mathrm{CO}_{2}$ and to crack $\mathrm{CH}_{4}$, while the second half of the getter remains at room temperature $\left(20^{\circ} \mathrm{C}\right)$ to sorb $\mathrm{H}_{2}$. The noble gases are inert to the getter pellets and flow through unimpeded.

The sample is next drawn for 12 minutes onto the SSC, held at less than 9.5 K. Neon, Ar, $\mathrm{Kr}$ and Xe are trapped on the stainless steel surface. Helium does not sorb quantitatively to the stainless steel surface at this temperature, so the sample is next exposed for 8 minutes to the ACC (Lott. and Jenkins, 1984) operated at $8.5 \mathrm{~K}$ in order to trap He. Meanwhile, the SSC is isolated and warmed to $40 \mathrm{~K}$ for 3 minutes and then cooled to $9.5 \mathrm{~K}$ in order to liberate the $2-4 \%$ of the He that was cryotrapped by Ar or the other gases. The ACC subsequently pumps on the SCC for 30 seconds in order to cryosorb this He onto the ACC.

The sample is next drawn for 12 minutes for a second time onto the SSC, while the inlet side of the WVC is warmed to $285 \mathrm{~K}$ and the outlet side of the WVC is held at $160 \mathrm{~K}$. The ice is thus melted and the water is distilled onto the outlet WVC, releasing any gases that had been trapped in the ice for subsequent purification and cryotrapping while blocking water vapor.

The SSC is warmed to $60 \mathrm{~K}$ and then is cooled back to $25 \mathrm{~K}$ in order to outgas and release Ne that had been cryotrapped by Ar. The SSC is then opened and Ne is released from the trap. The amount of Ne released is volumetrically split by a factor of roughly 200 in a reproducible fashion by trapping an aliquot of the released gas between valves 40 and 41 in order to obtain a sample size that gives a counting rate of about $140,000 \mathrm{cps}$, an optimal counting rate for the SEM, which is operated in the ion counting mode. Too high a counting rate leads to dead-time issues, a nonlinear response, and a short lifetime for the SEM. Too low a counting rate leads to poor Poisson ion-counting statistics. Because volume partitioning is used to split the $\mathrm{Ne}$, some fraction of Ne remains on the SSC. This is removed by ion pumping for 2 minutes at $20 \mathrm{~K}$, which is $5 \mathrm{~K}$ less than the release temperature in order to avoid any Ar from being pumped. 
The SSC is then warmed to $80 \mathrm{~K}$ in order to uniformly distribute Ar on its surface. Meanwhile, the ACC is warmed to $40 \mathrm{~K}$ and then is opened to release He. An aliquot of He, equal to approximately $1 \%$ of the sample is trapped between valves 38 and 39, is inlet into the QMS, and measured with a counting rate of about 100,000 cps on the SEM. Next, the $99 \%$ of the remaining He is volume partitioned into the HIMS where the ${ }^{3} \mathrm{He} /{ }^{4} \mathrm{He}$ ratio is measured.

The ACC is then warmed to $80 \mathrm{~K}$. An aliquot of gas is released from ACC and analyzed for Ne, in order to quantify any Ne that did not sorb to the SCC but rather made it through to the ACC. The amount of Ne measured from the ACC is approximately $5 \%$ of the amount measured from the SSC for gas standards and approximately $0.5 \%$ of the amount measured from the SSC for water samples, suggesting the SSC exhibits a variable $\mathrm{Ne}$ trapping efficiency which is dependent on major gas composition, including possibly water vapor. The $\mathrm{ACC}$ is cleaned by ion pumping at $80 \mathrm{~K}$ for 10 minutes.

Meanwhile, the SSC remains isolated and held at $80 \mathrm{~K}$ for two minutes, before it is slowly cooled to $25 \mathrm{~K}$ to recondense Ar on the cryotrap. The cooling is slower than usual in order to layer the $\mathrm{Kr}$ on the SSC first and then the Ar and thus to minimize commingling of $\mathrm{Ar}$ and $\mathrm{Kr}$. The SSC is warmed to $60 \mathrm{~K}$ and opened to release Ar. Because Ar is very abundant in air samples, the Ar sample must be reproducibly split down by roughly a factor of approximately $3 \times 10{ }^{6}$ in order to avoid overwhelming the QMS. Thus Ar is expanded for four minutes into a 2 liter stainless steel expansion volume, through valves 10, 9, 40, 42, 43 and 24. We performed experiments to determine that four minutes were required for the Ar atoms to have time to distribute evenly throughout the expansion volume. When the expansion time was shorter, the Ar signal increased, suggesting that the Ar had not reached equilibrium in the expansion volume, and thus fewer Ar atoms were pumped away, leaving more Ar to be analyzed.

Approximately $0.03 \%$ of the $\mathrm{Ar}$ is trapped in the aliquot between valves 42 and 43 and the remaining $\mathrm{Ar}$ is pumped away using a turbo molecular pump. The Ar in the aliquot is then expanded for a second time, this time into the volume bounded by valves 40, 43, and 9. Again, the Ar in the aliquot between valves 42 and 43 is saved and the rest is pumped away. Once more, the Ar is expanded into the volume bounded by valves 41,9 and 43 . This time the portion of the Ar in the aliquot between valves 40 and 41 is saved and inlet into the QMS. Although this splitting procedure may seem cumbersome, it enables the consistent splitting of Ar by a factor of approximately $3 \times 10^{6}$ with a reproducibility of $0.1 \%$. 
In order to remove any Ar remaining on the SSC, the SCC is then ion pumped at $62 \mathrm{~K}$ for two minutes. This pumping temperature is higher than the release temperature in order to remove enough Ar so as to minimize interference with the $\mathrm{Kr}$ measurements. Then, in order to remove any Ar that still remains on the SSC, perhaps cryotrapped under $\mathrm{Kr}$ or Xe, a temperature/pumping cycle is performed. The SSC is isolated and warmed to $150 \mathrm{~K}$, cooled back to $62 \mathrm{~K}$, and then ion pumped for 3 minutes. This heating and pumping cycles results in an acceptably low amount of Ar in our $\mathrm{Kr}$ and Xe samples - the Ar introduced into the QMS when the $\mathrm{Kr}$ is inlet produces an ion current that is about $20 \%$ of the $\mathrm{Kr}$ signal. It is possible to further reduce the amount of $\mathrm{Ar}$ in the $\mathrm{Kr}$ inlet by pumping for longer or at higher temperatures. However, this results in significantly greater $\mathrm{Kr}$ loss, resulting in deterioration of our $\mathrm{Kr}$ results.

The SSC is isolated, warmed to $103 \mathrm{~K}$ and opened to release $\mathrm{Kr}$. The $\mathrm{Kr}$ is split by a factor of approximately 130 by trapping an aliquot of sample between valves 40 and 41 and then is inlet into the QMS and measured on the SEM at an ion current of approximately $150,000 \mathrm{cps}$. The SSC is cooled to $93 \mathrm{~K}$ and then turbo pumped for 2 minutes to remove remaining Kr. The SSC next undergoes a heating/pumping cycle to remove any $\mathrm{Kr}$ cryotrapped by $\mathrm{Xe}$. The SSC is warmed to $150 \mathrm{~K}$, cooled back to $93 \mathrm{~K}$, and pumped on for 1 minute.

The SSC is next warmed to $155 \mathrm{~K}$ and opened to release Xe. The Xe is split by a factor of approximately 6 by expansion of the sample through valves $9,40,42$, and 43 and then trapping of a subsection in the section between valves 9, 41, and 42. This subsection is inlet into the QMS and measured on the SEM with an ion count rate of approximately $150,000 \mathrm{cps}$. The SSC is turbo pumped for 1.5 minutes to remove any remaining Xe. The SSC is then ion pumped while being warmed to 290 and pumped and held at 290 for five minutes. Next the ACC and the SSC are cooled to $10 \mathrm{~K}$ in order to prepare for the next sample.

The total analysis time for one sample is approximately three hours and 20 minutes and the procedure is completely automated. Line blanks are run every few days in order to check for leaks and to assess the cleanliness of the system. Line blanks typically are smaller than $0.004 \%$ for $\mathrm{He}, \mathrm{Kr}$, and $\mathrm{Xe}$, smaller than $0.01 \%$ for $\mathrm{Ne}$, and smaller than $0.07 \%$ for Ar. 


\subsubsection{Additional Steps for a Water Sample}

Water samples consist of $90 \mathrm{~g}$ of water taken at sea by gravity feeding water from Niskin bottles on a CTD rosette via Tygon tubing into valved stainless steel cylinders. In the on-shore laboratory at the Bermuda Biological Station, we extracted the gases from the cylinders into aluminosilicate glass bulbs (approximate volume of bulb is $25 \mathrm{cc}$ ) using the "at-sea extraction system" (Lott and Jenkins, 1998). The glass sample bulb contains $>99.95 \%$ of the original sample as well as 3 to $5 \mathrm{cc}$ of distilled water transferred during the extraction.

Because $\mathrm{He}$, and to a less extent $\mathrm{Ne}$, permeates through the viton o-rings in the cylinder plug valves, samples were extracted as soon as possible, usually within 24 hours of sample collection. This time period was comparable to the typical delays encountered in practice during the WOCE/CLIVAR sampling. Experiments performed with degassed water samples documented the rate at which samples are compromised. He and $\mathrm{Ne}$ were equilibrated at rates of $0.46 \%$ and $0.09 \%$ of their disqulibrium per day. Thus for a sample with a $10 \%$ disequilibrium in either $\mathrm{He}$ or $\mathrm{Ne}$ concentration or $\mathrm{He}$ isotope ratio, a 24 hour delay in extraction from the time of sampling would lead to a signal reduction of $0.046 \%, 0.009 \%$, and $0.046 \%$ respectively.

Sets of eight of these glass bulb samples are attached to the automanifold using viton o-ring compression fittings. The manifold is then pumped for at least 2 hours, a processing blank is run to assess for any leaks, and then the samples are individually processed, with one gas standard being run after every two water samples. To process a sample, first all of the sample sections except the sample of interest are closed off, and the manifold is isolated from the vacuum pumps and the rest of the processing line. Custom-fabricated automated "crackers", based on a heavily modified Nupro valve (P/N SS-6-6BK-TW-10), snap the tip of the glass seal-off on the bulb. Experimentation revealed that an optimum pneumatic pressure of 40 psi achieves reliable "cracking open" of the sample. On cracking of the bulb tip, the gas from the headspace of the sample bulb is partitioned into the volume of the manifold.

After 30 seconds, the manifold is opened and the gas expands into the dual WVC. Here, water is drawn out of the sample, quantitatively sweeping all the gas from the headspace out of the bulb into the WVC section of the processing line. After 1.5 minutes, the valve to the bulb is closed to avoid excessive water vapor transfer. Beyond this point, the sample gases are processed in an identical fashion to the standard gases. 
Table 2.1: Theoretical and experimentally determined amount of gas typically left behind in a sample bulb after the gas in the headspace is swept into the processing line for analysis. The ratios are different for each position on the manifold. One example set of ratios is listed here. The ratio of theoretical to measured left-behind is used to correct all samples for the total gas in the bulb. Uncertainties in this ratio thus lead to uncertainties in determinations of gas concentrations in samples.

\begin{tabular}{cccccc}
\hline & $\mathrm{He}$ & $\mathrm{Ne}$ & $\mathrm{Ar}$ & $\mathrm{Kr}$ & $\mathrm{Xe}$ \\
\hline Theoretical left-behind (\%) & 0.16 & 0.19 & 0.63 & 1.1 & 2.1 \\
Ratio of theoretical to measured left-behind & 0.87 & 0.98 & 0.79 & 0.62 & 0.85 \\
Estimated uncertainty in ratio & 0.07 & 0.1 & 0.05 & 0.03 & 0.03 \\
Uncertainty in final correction for samples (\%) & 0.01 & 0.01 & 0.03 & 0.01 & 0.06 \\
\hline
\end{tabular}

Some fraction of the gases are dissolved in water and thus not all the gas is drawn out of the bulb. This amount of gas left behind can be calculated theoretically from the volume and temperature of the water, the volume of the bulb, and the solubility of the gas. Additionally, it was determined experimentally on some samples by repeated drawing of gas from the same sample. More gas was consistently drawn from the samples than the theoretical calculation predicted, suggesting that some of the gas was fluxed out of the water during the draw (Table 2.1). The ratio of the measured sample left behind in the bulb to theoretical sample left behind in the bulb is consistent for each position on the manifold and is used to correct all measurements for the amount of gas left in the bulb. Manifold positions that are closer to the WVC have less gas left-behind because then the WVC is more effective at drawing out the gas. Samples that are on the lower manifold also have less gas left-behind because they are slightly warmed from below by the diffusion pump and gases are less soluble at warmer temperatures. We thus measure the ratio for each position on the manifold and correct each sample accordingly. This ratio is more difficult to calculate for $\mathrm{He}$ and $\mathrm{Ne}$ since $\mathrm{He}$ and Ne diffuse into the bulb during the time period between samples. We used repeated measurements in order to assess the amount of $\mathrm{He}$ and $\mathrm{Ne}$ that grows in but still our estimates for $\mathrm{He}$ and $\mathrm{Ne}$ left behinds have larger errors. However, since these gases are the least soluble, only a small percentage is left-behind and thus even with these larger uncertainties, the error added by the total correction is small.

To assess whether any gas was left behind in the manifold or WVC, experiments were done in which the 
manifold and WVC were isolated after a sample was drawn and processed. The WVC was warmed to 285 in order to melt the ice and release all gases and then cooled to $160 \mathrm{~K}$. The gas in the WVC and manifold was measured, using the the usual procedure, to be less than $0.005 \%$ of that contained in a sample. Thus the dual stage WVC sucessfully prevented gas from being trapped in the ice.

\subsubsection{Details of QMS Analysis}

Scanning and data collection on the QMS commences immediately prior to isolation and sample inlet. After the QMS is isolated from its ion pump, the gas samples are expanded into the QMS for a specified inlet time (Table 2.2), while the QMS continues measuring the ion current at the selected isotopes. After the specified inlet time finishes, valve 1 is closed and the QMS continues to measure the ion count of the isotopes for a total number of scans (Table 2.2), such that the overall analysis time is approximately 5 minutes. The QMS also measures the ion count of other gases that have the potential to be large enough to influence the results, such as $\mathrm{H}_{2}, \mathrm{CH}_{4}, \mathrm{CO}_{2}, \mathrm{H}_{2} \mathrm{O}$, and the other noble gases (Tables 2.3 and 2.4). Monitoring is done to ensure that these gases do not reach undesirable levels.

Measurement of all isotopes is made at specific mass points rather than by scanning individual peaks. Data reduction involves fitting the requisite scans as functions of time (discussed in section 2.2.6). The emission current was set for each gas in order to achieve a reasonable ion count rate (see Table 2.2). The ion count rate should be large enough to give good counting statistics but small enough so as to avoid degradation of the performance of the SEM. The voltage of the SEM was set to be at 2600, which is slightly above the "knee" in the curve of voltage vs. count rate.

\subsubsection{Details of Magnetic Sector Analysis}

The helium isotope sample is expanded into the HIMS for 30 seconds. After 30 seconds of settling time, the magnet current is adjusted so as to center the ${ }^{3} \mathrm{He}$ peak at an ion energy of $2844.6 \mathrm{~V}$ : for four times the ${ }^{3} \mathrm{He}$ counts are measured on the pulse-counting SEM at 2846.6 and at 2842.6 accelerating voltage and then the magnet current is adjusted so that the ion counts at those voltages are roughly equal. Since the Faraday cup has been placed so that the ${ }^{4} \mathrm{He}$ peak center aligns with the ${ }^{3} \mathrm{He}$ peak center, by centering on

${ }^{3} \mathrm{He}$, one also is in the center of the ${ }^{4} \mathrm{He}$ peak. Furthermore, the ${ }^{4} \mathrm{He}$ peak is much broader than the ${ }^{3} \mathrm{He}$ 
Table 2.2: Parameters used by the QMS to measure the five noble gases in a water sample or gas standard.

\begin{tabular}{cccccc}
\hline & $\mathrm{He}$ & $\mathrm{Ne}$ & $\mathrm{Ar}$ & $\mathrm{Kr}$ & $\mathrm{Xe}$ \\
\hline Isotopes Measured & ${ }^{4} \mathrm{He}$ & ${ }^{20} \mathrm{Ne},{ }^{22} \mathrm{Ne}$ & ${ }^{40} \mathrm{Ar},{ }^{36} \mathrm{Ar}$ & ${ }^{84} \mathrm{Kr},{ }^{86} \mathrm{Kr},{ }^{82} \mathrm{Kr}$ & ${ }^{132} \mathrm{Xe},{ }^{129} \mathrm{Xe},{ }^{136} \mathrm{Xe}$ \\
CryoTrap Used & $\mathrm{ACC}$ & $\mathrm{SSC} \& \mathrm{ACC}$ & $\mathrm{SSC}$ & $\mathrm{SSC}$ & $\mathrm{SSC}$ \\
Release Temp (K) & 40 & 25 & 60 & 103 & 155 \\
Splitting Factor & 100 & 200 & $3 \times 10^{6}$ & 130 & 6 \\
\# Scans by QMS & 200 & 90 & 110 & 90 & 130 \\
Emission( $\mu$ Amp) & 20 & 20 & 40 & 40 & 20 \\
Inlet Time (s) & 60 & 60 & 60 & 60 & 90 \\
\hline
\end{tabular}

peak so that misalignment is far less of a problem than for the ${ }^{3} \mathrm{He}$ peak. Next 10 cycles are conducted in which typically the peak is measured 11 times with 20 second integrations each time, bracketed by a 20 second baseline measurement at an accelerating voltage of $2854.6 \mathrm{~V}$ to determine the scattering background signal. A typical standard has a ${ }^{3} \mathrm{He}$ ion current of $\sim 1500 \mathrm{cps}$, and a typical background of $18 \mathrm{cps}$. With the aforementioned counting times, a sample would typically accumulate $3.3 \times 10^{6}$ counts on the ${ }^{4} \mathrm{He}$ peak and 2000 counts on the baseline. Thus the Poisson counting limitations to the precision of the isotope ratio measurement would be $0.06 \%$. The count rate at HD and the baseline are measured once again and then the gas is pumped away through valve 0 by an ion pump. Overall the analysis takes about 45 minutes and is performed while the processing line and QMS are measuring the other noble gases in the sample.

\subsubsection{Computer Control}

All processes are automated in order to achieve reproducible, "around-the-clock" measurements. The gateway to the physical system is a visual basic program, the "Server", run on the Dell Windows XP computer, which is directly integrated into the instrumentation. The server controls all the pneumatic valves, sends commands to the HIDEN QMS, controls the HIMS, and measures the pressures and temperatures of all the components of the processing line. The Server also manages a common pool of boolean flags that are used 
Table 2.3: Isotopes and other masses measured on the QMS during the analysis of $\mathrm{He}, \mathrm{Ne}$, and Ar: typical count rates for a standard, dwell times, and Poisson ion counting statistics. Negative count rates are clearly unphysical and are a result of extrapolation to end of inlet for small count rates for some of the masses in the blanks.

\begin{tabular}{|c|c|c|c|c|c|c|c|c|c|c|c|c|c|}
\hline Helium & & & & & & & & & & & & & \\
\hline Masses & 3.9 & 2 & 5 & 16 & 18 & 19 & 20 & 28 & 32 & 40 & 44 & & \\
\hline dwell time (s) & 1000 & 100 & 100 & 100 & 100 & 100 & 100 & 100 & 100 & 100 & 100 & & \\
\hline std cps & 97,000 & 100,000 & 0.56 & 69,000 & 370 & 11,000 & 200 & 2200 & 2.6 & 66 & 800 & & \\
\hline Poisson (\%) & 0.02 & & & & & & & & & & & & \\
\hline blank cps & 3 & 110,000 & 0.1 & 74,000 & 390 & 10,000 & 15 & 2100 & 3 & 49 & 580 & & \\
\hline \multicolumn{14}{|l|}{ Neon } \\
\hline Masses & 20 & 22 & 2 & 4 & 5.5 & 14 & 16 & 18 & 19 & 28 & 32 & 40 & 44 \\
\hline dwell time (s) & 500 & 1000 & 50 & 100 & 100 & 50 & 50 & 50 & 50 & 50 & 50 & 100 & 100 \\
\hline std cps & 140,000 & 13,000 & 61,000 & 56 & 0.016 & 570 & 8900 & 390 & 11,000 & 1400 & 2.7 & 79 & 690 \\
\hline Poisson (\%) & 0.05 & 0.11 & & & & & & & & & & & \\
\hline blank cps & 13 & 3 & 63,000 & -0.4 & -17 & 490 & 7900 & 410 & 11,000 & 1300 & 8 & 16 & 564 \\
\hline \multicolumn{14}{|l|}{ Argon } \\
\hline masses & 40 & 36 & 2 & 5 & 16 & 19 & 20 & 22 & 28 & 32 & 44 & & \\
\hline dwell time (s) & 100 & 800 & 100 & 600 & 100 & 100 & 100 & 100 & 100 & 100 & 100 & & \\
\hline std cps & 110,000 & 400 & 240,000 & 0.73 & 25,000 & 19,000 & 13,000 & 21 & 3800 & 7.5 & 2300 & & \\
\hline Poisson (\%) & 0.04 & 0.69 & & & & & & & & & & & \\
\hline blank cps & 93 & 3 & 250,000 & 0.4 & 24,000 & 19,000 & 25 & 19 & 3500 & 8 & 2100 & & \\
\hline
\end{tabular}


Table 2.4: Isotopes and other masses measured on the QMS during the analysis of $\mathrm{Kr}$ and $\mathrm{Xe}$ : typical count rates for a standard, dwell times, and Poisson ion counting statistics. Negative count rates are clearly unphysical and are a result of extrapolation to end of inlet for small count rates for some of the masses in the blanks.

\begin{tabular}{|c|c|c|c|c|c|c|c|c|c|c|c|c|c|}
\hline \multicolumn{14}{|l|}{ Krypton } \\
\hline masses & 84 & 86 & 82 & 2 & 5.5 & 16 & 18 & 19 & 28 & 32 & 40 & 44 & 132 \\
\hline dwell time(s) & 600 & 600 & 600 & 100 & 100 & 100 & 100 & 100 & 100 & 100 & 100 & 100 & 100 \\
\hline std cps & 134,000 & 38,000 & 27,000 & 240,000 & 0.75 & 26,000 & 620 & 20,000 & 4400 & 6.9 & 15,000 & 2100 & 25 \\
\hline Poisson (\%) & 0.04 & 0.08 & 0.09 & & & & & & & & & & \\
\hline blank cps & 0.7 & 0.3 & 0.4 & 240,000 & 0.7 & 23,000 & 630 & 19,000 & 4000 & 5.9 & 5200 & 1900 & 1.9 \\
\hline \multicolumn{14}{|l|}{ Xenon } \\
\hline masses & 132 & 129 & 136 & 2 & 5.5 & 16 & 18 & 19 & 28 & 32 & 40 & 44 & 84 \\
\hline dwell time (s) & 600 & 600 & 600 & 100 & 100 & 100 & 100 & 100 & 100 & 100 & 100 & 100 & 300 \\
\hline std cps & 130,000 & 120,000 & 40,000 & 100,000 & 0.41 & 36,000 & 330 & 9100 & 1700 & 3.2 & 320 & 690 & 7600 \\
\hline Poisson (\%) & 0.04 & 0.04 & 0.06 & & & & & & & & & & \\
\hline blank cps & 1.1 & 0.7 & 0.2 & 100,000 & -0.2 & 54,000 & 320 & 9000 & 1600 & 2 & 69 & 610 & 1.9 \\
\hline
\end{tabular}


to coordinate various client programs. When the system is in normal analysis mode, a client program know as "Menu" is used to schedule and initiate sample or standard analyses. The Menu program uses a series of code numbers to signify various characteristics of each procedure, and on schedule launches another client program, called "Procline" that executes the analysis. Procline operates by sending commands to the Server to operate valves, warm and cool the cryotraps, start analysis on the mass spectrometers, etc. in a reproducible fashion as well as to record system conditions (pressure, temperature, etc) to disk file during the analysis. Analysis of the noble gases on the QMS is also accomplished by Procline, which downloads scan control information and various commands to an embedded computer on the QMS (MSIU) and uploads acquired measurement data. Helium isotope measurements on the HIMS are made by another client program called HeAnal that is launched at the appropriate time by Procline. Data from the HIMS is collected using counter-timer cards on the core computer: the ${ }^{4} \mathrm{He}$ is measured by a Faraday cup electrometer that is digitized using a $10 \mathrm{KHz}$ voltage-to-frequency converter, and the ${ }^{3} \mathrm{He}$ ions are counted using a channeltron electron multiplier and a preamplifier/discriminator unit.

In addition there are other programs that are used as "utilities" from time to time. For example, a virtual "Chart Recorder" can display and save readouts of the temperature or pressures of the various gauges. Also, a general purpose scripting program called "Tweak" can be used to execute a range of useful procedures on the system. Under normal conditions, 16 samples can be loaded onto the two manifolds, the Menu set up, and then the system can operate completely independently for approximately 5 days (or longer if just gas standards are being analyzed). Monitoring of all components and changes to the programs can be done remotely (i.e. from the Internet). Data is stored by these programs in a hierarchical directory structure using data-encoded file names.

\subsubsection{Standardization}

After the analysis of the sample is complete, a MATLAB routine is used to linearly extrapolate the mass peak ion count rate from all the scans back to the end of the inlet in order to calculate a single representative ion count rate associated with the amount of a given isotope in a sample or standard. The ion count rate of a sample is quantitatively compared to the ion count rate in a standard (std) in order to calculate the concentration of gas in a sample: 
cc of gas in sample $=$ cc of gas in std $\times \frac{\text { sample ion count rate }- \text { blank ion count rate }}{\text { std ion count rate }- \text { blank ion count rate }}$

where "blank ion count rate" refers to the ion count rate measured when running a wet-blank - running the same procedure but without introducing sample. We are explicitly assuming a linear relationship between ion count rate and the pressure of the gas and hence the sample size. Deviations from this relationship are determined empirically and corrected for (see section 2.3.1).

For a standard, we use aliquots of a sample of marine air that was collected in a 15 liter metal can on the beach outside of Woods Hole Oceanographic Institution. The metal can was first evacuated in the lab to a pressure of $1 \times 10^{-7}$ torr. The can was then brought to the beach, equilibrated with atmospheric conditions for 1 hour, and filled with air, with the temperature, relative humidity, and barometric pressure being recorded. Each time a standard is run, the remaining amount of gas in the standard tank is depleted by $5 \times 10^{-5}$ or $5 \times 10^{-6}$, depending on the size of the aliquot of standard removed. This depletion factor is taken into account when calculating results. There are two aliquot volumes connected to this running sample, one being $10 \%$ the size of the other, in order to calculate linearity effects associated with larger or smaller gas sizes. The amount of gas in each aliquot is listed in Table 2.5.

The running standard is calibrated against a reference air standard - an air standard collected in the same way but only run occasionally in order to assess if leaks or other problems have developed with the running standard. The air standards and reference air standards typically agree within $0.2 \%$ and multiple reference air standards are calibrated against a running standard to decrease the error further.

The amounts of $\mathrm{Ar}, \mathrm{Kr}$ and $\mathrm{Xe}$ in a water sample differ from those in an air standard by roughly a factor of 2, 4, and 7 respectively. Thus, we created a "makeup" standard - a standard consisting of purchased, purified $\mathrm{Ar}, \mathrm{Kr}$, and $\mathrm{Xe}$ in an amount such that one aliquot of makeup standard plus one aliquot of the air standard would result in $\mathrm{Ar}, \mathrm{Kr}$, and Xe concentrations similar to that of a water sample. Thus by running one aliquot of the makeup standard and one aliquot of regular air standard, we have similar gas concentrations and ratios in our standard as in our warm (shallow) water samples. By running two aliquots of the makeup standard and one aliquot of regular air standard, we have similar gas concentrations and ratios as in our cold (deep) water samples.

We made the makeup standard by expanding aliquots of pure $\mathrm{Xe}, \mathrm{Kr}$, and then $\mathrm{Ar}$ (in that order) at a 
Table 2.5: Amount of gas (in cc STP) in one aliquot of each type of gas standard. Small and large refer to two different size aliquots attached to the same air standard. Makeup refers to a standard comprised of only $\mathrm{Ar}, \mathrm{Kr}$, and $\mathrm{Xe}$ used to make the gas ratios in a total gas standard equivalent to that in a water sample.

\begin{tabular}{cccc}
\hline Isotope & Large & Small & Makeup \\
\hline 4 & $3.60 \times 10^{-6}$ & $3.80 \times 10^{-7}$ & 0 \\
20 & $1.14 \mathrm{E \times} 10^{-5}$ & $1.20 \times 10^{-6}$ & 0 \\
22 & $1.12 \times 10^{-6}$ & $1.19 \times 10^{-7}$ & 0 \\
40 & $6.42 \times 10^{-3}$ & $6.77 \times 10^{-4}$ & $1.15 \times 10^{-2}$ \\
36 & $2.18 \times 10^{-5}$ & $2.30 \times 10^{-6}$ & $3.90 \times 10^{-5}$ \\
84 & $4.46 \times 10^{-7}$ & $4.71 \times 10^{-8}$ & $1.83 \times 10^{-6}$ \\
86 & $1.33 \times 10^{-7}$ & $1.40 \times 10^{-8}$ & $5.47 \times 10^{-7}$ \\
82 & $9.08 \times 10^{-8}$ & $9.58 \times 10^{-9}$ & $3.73 \times 10^{-7}$ \\
132 & $1.61 \times 10^{-8}$ & $1.70 \times 10^{-9}$ & $1.27 \times 10^{-7}$ \\
129 & $1.58 \times 10^{-8}$ & $1.66 \times 10^{-9}$ & $1.25 \times 10^{-7}$ \\
136 & $5.32 \times 10^{-9}$ & $5.61 \times 10^{-10}$ & $4.21 \times 10^{-8}$ \\
\hline
\end{tabular}


known pressure and temperature into a $15 \mathrm{~L}$ standard tank that had previously been evacuated. The pressure was measured with a capacitance manometer to within 0.1 torr (MKS baratron controller, model 270B-4 with model 390HA 100 torr absolute pressure transducer). The Ar was ultra-high purity Ar from CorpBrothers. The $\mathrm{Kr}$ and Xe were research grade gases, $99.999 \%$ purity, from SpectraGas. We then calibrated the makeup standard for $\mathrm{Ar}, \mathrm{Kr}$, and $\mathrm{Xe}$ to better than $0.1 \%$ by running one aliquot of makeup standard and analytically comparing it to runs of two, four, or seven aliquots of the air standard. We ran multiple aliquots of air standards so as to match the size between the makeup and air standards as closely as possible to mitigate linearity effects. In addition, since the amount of $\mathrm{Ar}$ and $\mathrm{Xe}$ in the sample affects the amount of $\mathrm{Kr}$ released from the trap (see section 2.4.2), we used extra aliquots of pure $\mathrm{Ar}$ and $\mathrm{Xe}$ when calibrating for $\mathrm{Kr}$ to minimize this matrix effect. The amount of gas in one aliquot of the "makeup" standard is listed in Table 2.5.

\subsection{Analytical Performance and Reproducibility}

\subsubsection{Performance of the QMS and Processing Line}

By using both a charcoal and a stainless steel cryotrap, this method successfully separates the noble gases, such that for a measurement at a given release temperature, a sample is predominantly comprised of a single noble gas. The release curves for the gases from the stainless steel trap are depicted in Fig. 2-3. Good separation is obtained for $\mathrm{Ne}$ and $\mathrm{Ar}$. For $\mathrm{Ar}, \mathrm{Kr}$, and $\mathrm{Xe}$, however, the release curves overlap, making separation more difficult. Hence Ar and Kr have to be released below the temperature at which 100\% of the gas is released. In essence, the trade-off is between less quantitative release (and hence potentially greater temperature dependence and variability) vs. systematic effects associated with commingling gases. Krypton is released at $103 \mathrm{~K}$, but then the excess $\mathrm{Kr}$ in the trap is pumped away at a lower temperature $(93 \mathrm{~K})$ in order to prevent Xe from being dragged away with the Kr. We saw a 5\% increase in the amount of Xe when the pumping temperature for $\mathrm{Kr}$ was dropped from $103 \mathrm{~K}$ to $93 \mathrm{~K}$.

Figure 2-4 depicts the inlet curves at each of the five release temperatures for the noble gases and their isotopes in an air standard. The inlet curves are flat after the initial inlet, signifying there is little, if any, time dependence to the measurements. The isotope ${ }^{36} \mathrm{Ar}$ is also measured but not plotted because the signal 
Figure 2-3: Release curves of $\mathrm{Ne}, \mathrm{Ar}, \mathrm{Kr}$ and $\mathrm{Xe}$ from the stainless steel cryotrap as determined by analyzing repeated air standards at different release temperatures. At $9 \mathrm{~K}$, virtually none of the gases are released and thus all the gases are drawn onto the trap. Then as the trap temperature is slowly warmed, the gases are released one-by-one. The release curves of $\mathrm{Ne}$ and Ar have good separation. In contrast, the release curves of $\mathrm{Ar}, \mathrm{Kr}$, and $\mathrm{Xe}$ overlap slightly making these gases harder to separate.

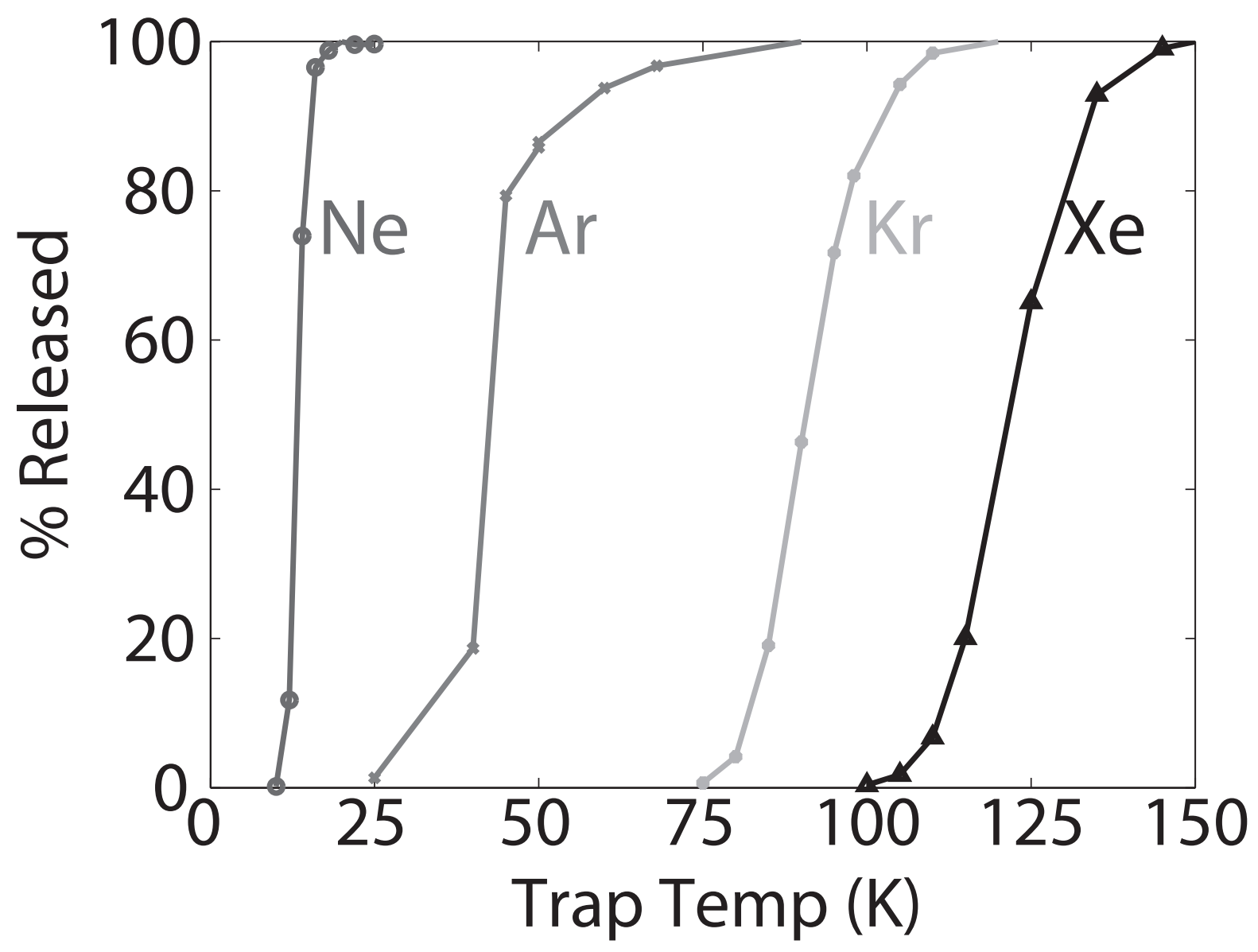


is small compared to that of ${ }^{40} \mathrm{Ar}$. In the $\mathrm{He}, \mathrm{Ne}$, and Ar inlets, only $\mathrm{He}, \mathrm{Ne}$, and Ar were detectable. In the $\mathrm{Kr}$ inlet, an amount of Ar equal to $20 \%$ of the $\mathrm{Kr}$ signal was detectable (Tab 2.4). In the Xe inlet, small amounts of $\mathrm{Ar}$ and $\mathrm{Kr}$ were detectable $(0.3 \%$ and $10 \%$ of the Xe signal respectively). Such small amounts of other noble gases should not interfere with the measurements of the primary noble gas. When measuring ${ }^{20} \mathrm{Ne}$ and ${ }^{22} \mathrm{Ne}$, one needs to also consider any contribution from doubly-charged ${ }^{40} \mathrm{Ar}$ or ${ }^{44} \mathrm{CO}_{2}$, as such species will have the same mass as ${ }^{20} \mathrm{Ne}$ and ${ }^{22} \mathrm{Ne}$. Assuming $10 \%$ of the ${ }^{40} \mathrm{Ar}$ and $1 \%$ of the ${ }^{44} \mathrm{CO}_{2}$ is doubly charged, then $\mathrm{Ar}$ and $\mathrm{CO}_{2}$ could be contributing to $0.004 \%$ and $0.1 \%$ of the ${ }^{20} \mathrm{Ne}$ and ${ }^{22} \mathrm{Ne}$ signals respectively (Tab 2.3). Such a contribution is corrected for, as $\mathrm{Ar}$ and $\mathrm{CO}_{2}$ are measured every time.

The isotopic ratios of $\mathrm{Ne}, \mathrm{Ar}, \mathrm{Kr}$ and $\mathrm{Xe}$ as measured by the QMS and in the atmosphere are listed in Table 2.6. The ratio of ${ }^{22} \mathrm{Ne} /{ }^{20} \mathrm{Ne}$ measured by the QMS is smaller than the ratio in air, suggesting that this method (either the QMS or the processing) may be discriminating against the heavier isotope. Similarly, the ratio of ${ }^{86} \mathrm{Kr} /{ }^{84} \mathrm{Kr}$ and ${ }^{136} \mathrm{Xe} /{ }^{132} \mathrm{Xe}$ suggest discrimination against the heavier isotope. However, the ratios of ${ }^{84} \mathrm{Kr} /{ }^{82} \mathrm{Kr}$ and ${ }^{132} \mathrm{Xe} /{ }^{129} \mathrm{Xe}$ are larger than the ratio in air, suggesting discrimination against the lighter isotope. Perhaps the discrimination is always against the less abundant isotope, instead of based on mass. The ion optics of a 3 stage quadrupole mass filter are extremely complicated and thus the mass discrimination function for the QMS as a whole can be very convoluted. There are a number of factors, including sample processing and release from the cryotraps that can contribute to "non-canonical" isotope ratios. Thus we rely on calibration and stability of the system - as long as the discrimination occurs equally in both standards and samples, then such a bias should not prevent the measurement of accurate isotopic ratios.

The peak shapes of the noble gases show clear separation of the isotopes (Fig 2-5). Seven isotopes of $\mathrm{Xe}$ and five isotopes of $\mathrm{Kr}$ can be distinguished in the appropriate mass range. The peaks are sloped, being steeper on the high mass side than the low mass side. This shape is typical of quadrupole mass filters and reflects imbalances between the low mass and high mass filters. The Hiden QMS used here has a prefilter and a postfilter on either side of the main mass filter, whose function it is to steer all the ions into an aligned beam for the main mass filter. To improve the peak shape, one needs to either increase the amount of time the ion beam is in the mass filter (by decreasing the speed at which the ions travel) or to increase the RF frequency so that the ions see more RF cycles. The latter can only be done by Hiden personnel at the 
Figure 2-4: Ion count rate of the mass peaks during the inlets of the noble gases. The He inlet occurred at release temperature of $40 \mathrm{~K}$ from the ACC. The other gases were released from the SSC at temperatures of $25 \mathrm{~K}, 60 \mathrm{~K}, 103 \mathrm{~K}$, and $155 \mathrm{~K}$ for $\mathrm{Ne}, \mathrm{Ar}, \mathrm{Kr}$ and Xe respectively. Isotopes of each noble gas that were measured are shown except for ${ }^{36} \mathrm{Ar}$ which is too small to be visible on the same plot as ${ }^{40} \mathrm{Ar}$. In the $\mathrm{Kr}$ and Xe inlets, other noble gases were detectable and thus are plotted as well. Inlet curves of the gases are labeled according to the mass of the gases, with mass 44 referring to $\mathrm{CO}_{2}$.
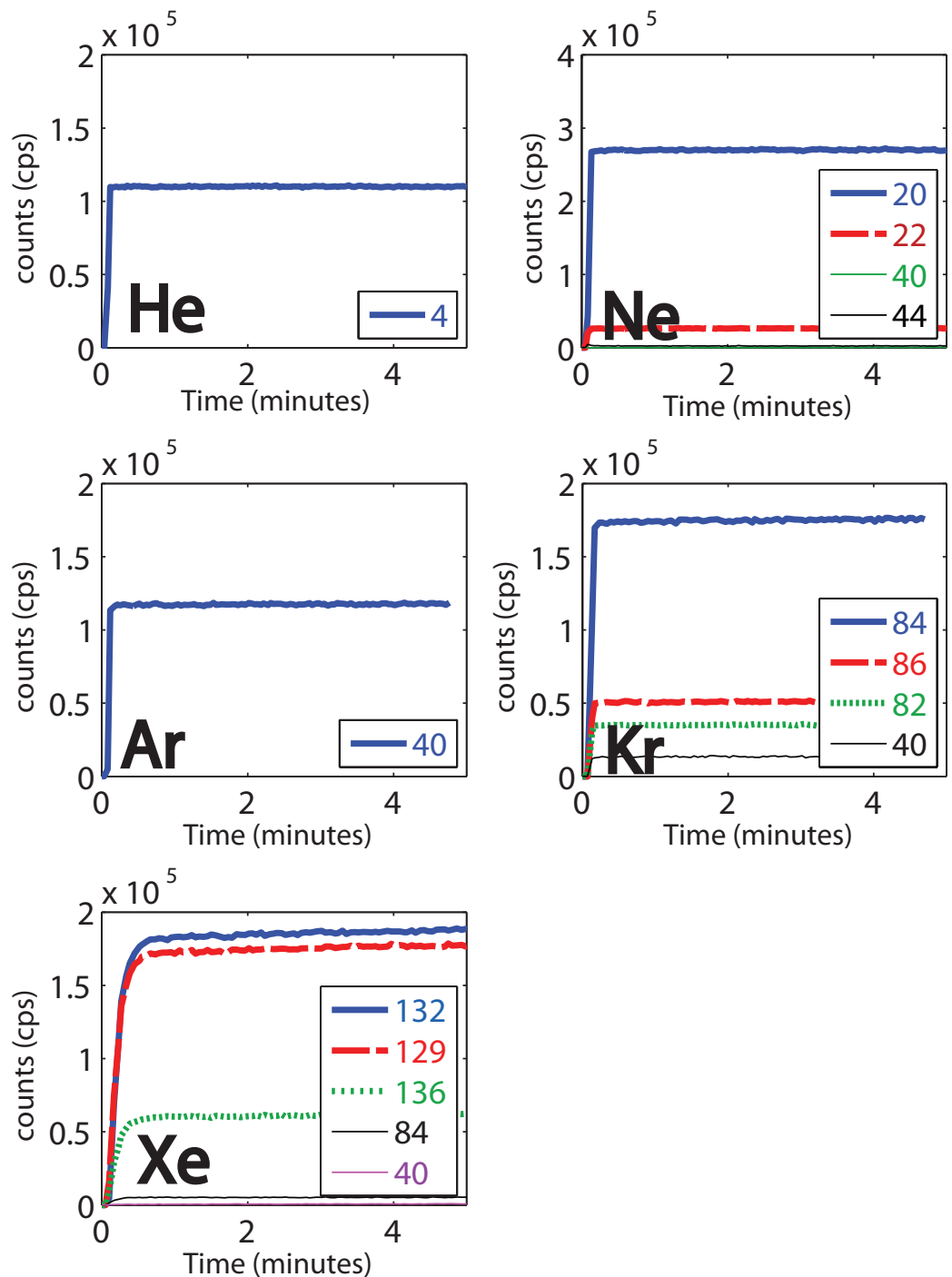
Table 2.6: Performance of the QMS for measuring the major isotopes of the five noble gases.

\begin{tabular}{cccccc}
\hline & ${ }^{4} \mathrm{He}$ & ${ }^{20} \mathrm{Ne}$ & ${ }^{40} \mathrm{Ar}$ & ${ }^{84} \mathrm{Kr}$ & ${ }^{132} \mathrm{Xe}$ \\
\hline Gas in a standard (cm $\left.{ }^{3} \mathrm{STP}\right)$ & $4.0 \times 10^{-6}$ & $1.3 \times 10^{-5}$ & $1.9 \times 10^{-2}$ & $2.3 \times 10^{-6}$ & $1.5 \times 10^{-7}$ \\
Signal on QMS (cps) & $1.1 \times 10^{5}$ & $2.7 \times 10^{5}$ & $1.2 \times 10^{5}$ & $1.7 \times 10^{5}$ & $0.17 \%$ \\
Precision (\%) & $0.10 \%$ & $0.14 \%$ & $0.10 \%$ & $0.14 \%$ & $0.004 \%$ \\
Blank (as \% of standard) & $0.004 \%$ & $0.01 \%$ & $0.07 \%$ & $0.0004 \%$ & \\
Isotopic ratios measured & - & & & & \\
Precision (\%) & - & $0.19 \%$ & $1.3 \%$ & $0.20,0.25 \%$ & $0.09,0.13 \%$ \\
Isotopic ratio in air & - & 0.102 & 293 & $0.304,4.91$ & $1.02,0.331$ \\
Isotopic Ratio from QMS & - & 0.098 & 271 & $0.291,4.95$ & ${ }^{129} \mathrm{Xe},{ }^{136} \mathrm{Xe} /{ }^{132} \mathrm{Xe}$ \\
\hline
\end{tabular}


Hiden facility so we did not pursue that route. The former can be done by lowering the cage voltage. We investigated the response of the peak shape to the cage voltage and indeed saw an improved peak shape with lower voltages (Fig 2-6). However lower voltages also led to decreased sensitivity. The optimal cage voltage was deemed to be $2 \mathrm{~V}$.

Because the peaks are not "flat topped" there are two potential sources of error that can be introduced into the analyses. First, because the measurement scans are done by setting the mass filter to one specific value for each isotope, then a change in the "registration" of this setting can lead to a shift in the apparent response of the instrument. Second, if the shape of the peak is dependent on the pressure of gas within the machine (due to scattering of ions off neutrals) then the apparent response will also be affected. We evaluate these effects by frequent standardization (using the assumption that the mass registration will change gradually with time), and by performing "linearity" curves with multiple standard aliquots.

Because the size of the standard is not exactly the same size as that of the sample, we assessed the linearity of the response of the QMS and of the sample/standard processing to the size of the samples. We analyzed standards with sizes varying from $90 \%$ to $200 \%$ of the typical standard size by composing a standard with varying combinations of small and large aliquots and varying amount of makeup standard. The response of the QMS to standard size is linear, with $r^{2}$ values of at least 0.999 . The linear response of the QMS throughout this range confirms that our samples and standards are in the linear dynamic range of the instrument. We used a least squares regression to fit the relative count rate between different size standards to the relative amount of gas in the standard, fixing the intercept to be 1. The slopes of $\mathrm{Ne}$, Ar, $\mathrm{Kr}$, and $\mathrm{Xe}$ are between 0.99 and 1.00, suggesting a straight-forward relationship between count rate and amount of gas in the sample. The slope of $\mathrm{Kr}$ has the greatest deviation from 1. The $\mathrm{Kr}$ measurements are affected by the amount of Ar and Xe in the sample. We thus need to take into account the effect of greater $\mathrm{Ar}$ and greater Xe when calculating the linearity. A full treatment of the $\mathrm{Kr}$ linearity and $\mathrm{Ar}$ and $\mathrm{Xe}$ matrix effect on $\mathrm{Kr}$ is described in section 2.4.2.

We examined the drift of the instrument due to factors such as changing room temperature, change in the SEM, change in filament, changing reactivity of the surface of the traps, etc. by making repeated measurements of the air standard over the course of several days (Fig 2-7). This long run had standard deviations of the measurements equal to $.09 \%$ for $\mathrm{He}, 0.15 \%$ for $\mathrm{Ne}, 0.23 \%$ for $\mathrm{Ar}, 0.31 \%$ for $\mathrm{Kr}$ and $0.42 \%$ 
Figure 2-5: The peak shapes for the isotopes measured on the QMS of each of the noble gases are plotted in arbitrary units. Five isotopes of $\mathrm{Kr}$ can clearly be seen as can seven isotopes of Xe. The peak shapes are more steeply sloped on the high mass side due to differences in the low and high mass filters.
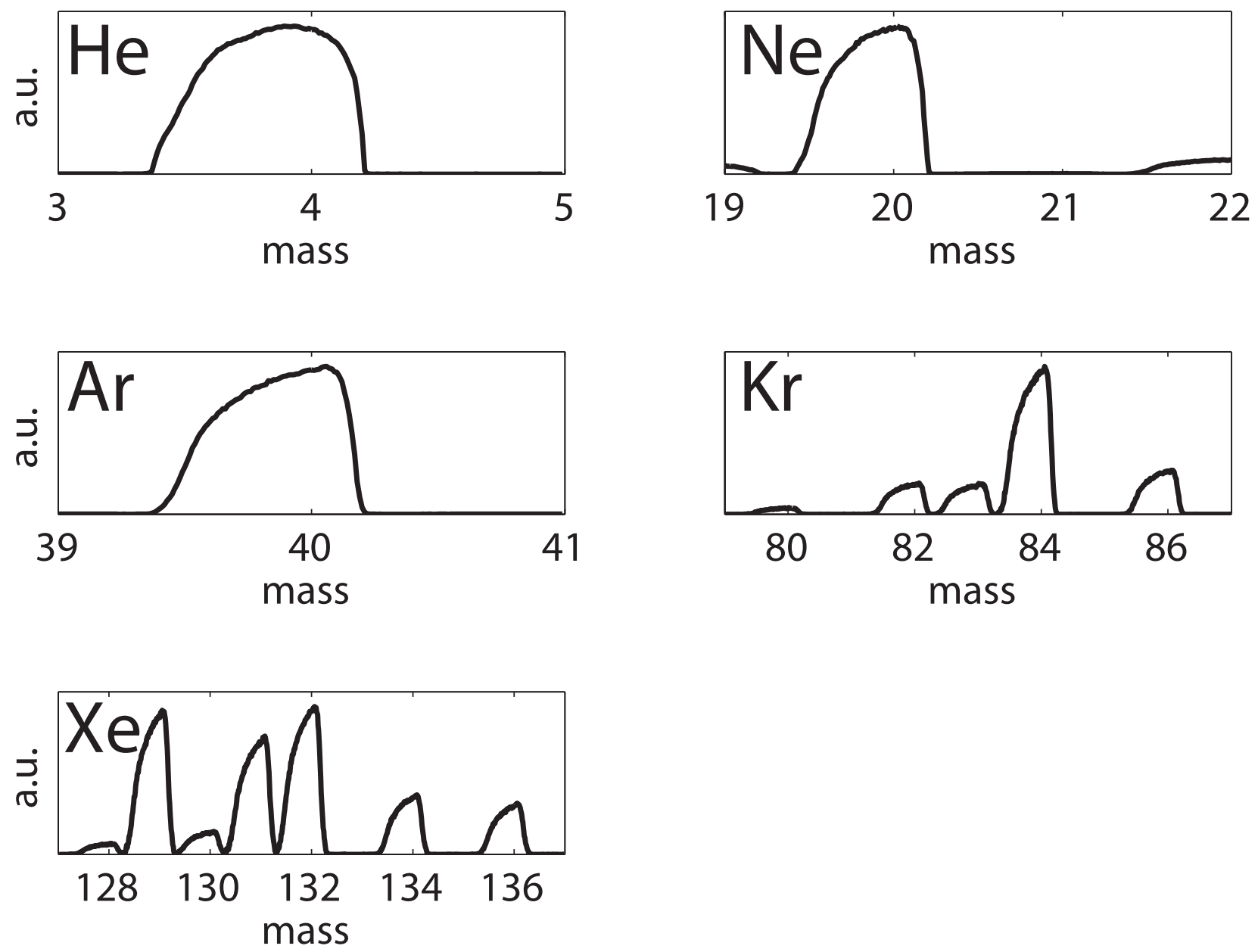
Figure 2-6: The peak shapes for the major isotope of each of the noble gases at different cage voltages ranging from $1.5 \mathrm{~V}$ (blue) to $3.5 \mathrm{~V}$ (red) are plotted in arbitrary units. The curves have been shifted vertically for clarity. As the cage voltage decreases, the top of the peak is flatter and the resolution improves but the sensitivity decreases. A cage voltage of $2 \mathrm{~V}$ was deemed to provide the best balance between sensitivity and peak shape.
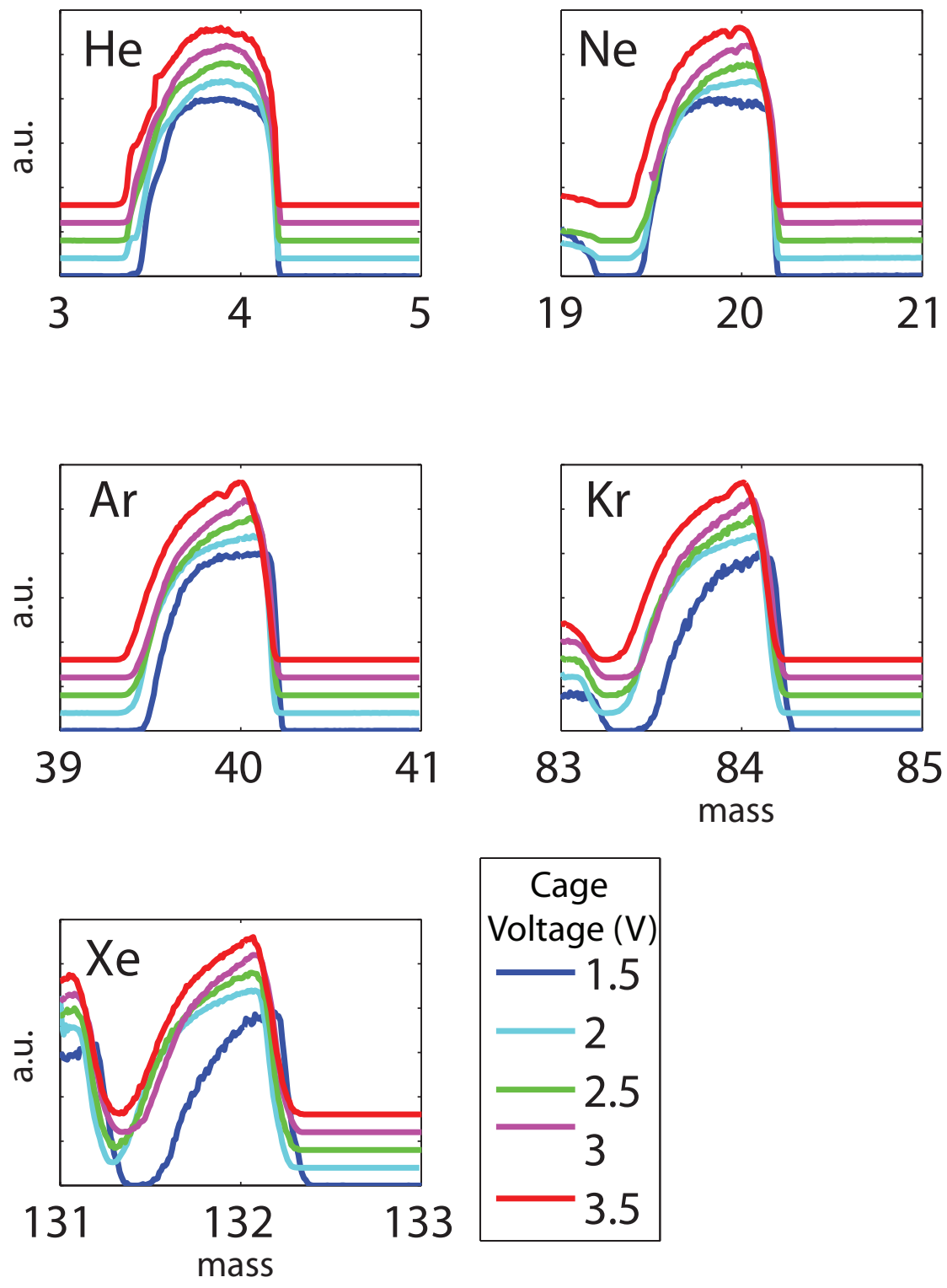
Table 2.7: Linearity of the QMS measurements. The linear dynamic range of the QMS and processing line was explored by processing standards ranging from $90 \%$ to $200 \%$ of the size of the typical gas standards. The data was then fit in a least-squares sense to a linear function with a fixed intercept of 1 . The slopes as determined by fit as well as their associated uncertainties are listed here.

\begin{tabular}{ccc}
\hline Isotope & Slope & $1 \sigma$ \\
\hline${ }^{4} \mathrm{He}$ & 0.995 & 0.001 \\
${ }^{20} \mathrm{Ne}$ & 0.995 & 0.01 \\
${ }^{22} \mathrm{Ne}$ & 0.998 & 0.009 \\
${ }^{40} \mathrm{Ar}$ & 0.997 & 0.001 \\
${ }^{36} \mathrm{Ar}$ & 1.006 & 0.005 \\
${ }^{84} \mathrm{Kr}$ & 1.027 & 0.003 \\
${ }^{86} \mathrm{Kr}$ & 1.022 & 0.003 \\
${ }^{82} \mathrm{Kr}$ & 1.021 & 0.003 \\
${ }^{132} \mathrm{Xe}$ & 0.994 & 0.001 \\
${ }^{129} \mathrm{Xe}$ & 0.995 & 0.002 \\
${ }^{136} \mathrm{Xe}$ & 0.984 & 0.002 \\
\hline
\end{tabular}


for Xe. The standards show a downward drift for all gases, that could be associated with aging of the filament and ion source, change in gain on the SEM, changes in room temperature, fluctuations in power source, etc. A quadratic trendline is fit to these measurements and deviations from the quadratic trendline a crude measure of the random uncertainty in the standard measurements - are calculated (Fig. 2-8).

Because of the downward drift, when measuring samples we alternately measure samples and standards and then use the interpolated standard results to calculate the sample values. Therefore, our objective is to determine the uncertainty associated with using the standards to predict a value for a sample measured between adjacent standards. In order to assess this, we treated half of the standards as "unknowns." We then fit a least squares smoothing spline to the remaining standards to interpolate the response of the system at the time of the "unknowns." By comparing the predicted value to the actual value, we can calculate the random error in our measurements. The thus computed errors ranged from $0.10 \%$ for $\mathrm{He}$ to $0.17 \%$ for $\mathrm{Xe}$ (Table 2.6).

A number of water samples prepared from a 10 liter container of water that was stirred for 24 hours while in contact with lab air were analyzed in order to assess the accuracy of the method. The average saturation state of the samples was calculated from the freshwater solubility values of Krause and Benson (1989) and are reported in Table 2.8. Helium and neon may be supersaturated due to bubble entrainment during the sampling procedure. The geometry of the plug valves on the sampling cylinder may be conducive to trapping a small quantity of air. The standard deviation of these samples ranged from $0.7 \%$ to $1.2 \%$, possibly reflecting variability in the samples themselves or in the preparation or in the analysis. This test was conducted before our method for analyzing all the noble gases was optimal; the method has improved significantly for Ne, $\mathrm{Kr}$, and $\mathrm{Xe}$ after this experiment was completed. Unfortunately, the water container was not located in a constant temperature bath. A 1 degree change in temperature results in a 3\% change in Xe saturation state so some of the variability in the Xe measurements may be due to temperature inhomogeneities in the bulk sample. In addition, the relative humidity and the barometric pressure in the room were not monitored and 24 hours may not have been enough time for full equilibration. In a future experiment, one should stir the water in a temperature controlled bath for at least one week, keep the water in contact with outside air (to avoid possible changes in gas ratios due to lab air such as a nearby liquid Ar tank or He carrier gas in a gas chromotograph), and record the relative humidity and barometric pressure at all times. Additionally, since 
Figure 2-7: The stability of the QMS measurements with respect to time was assessed by repeatedly running standards over several days (black circles). A quadratic trend line of count rates of the standards as a function of time is shown in gray. The full scales of each plot reflects a $2 \%$ range of the signal size. All the gases show a downward drift, with Xe having the steepest drift. This may be due to aging of the ion source or the electron multiplier. Histograms of deviations of the measurements from the quadratic trend line are shown in Fig. 2-8).
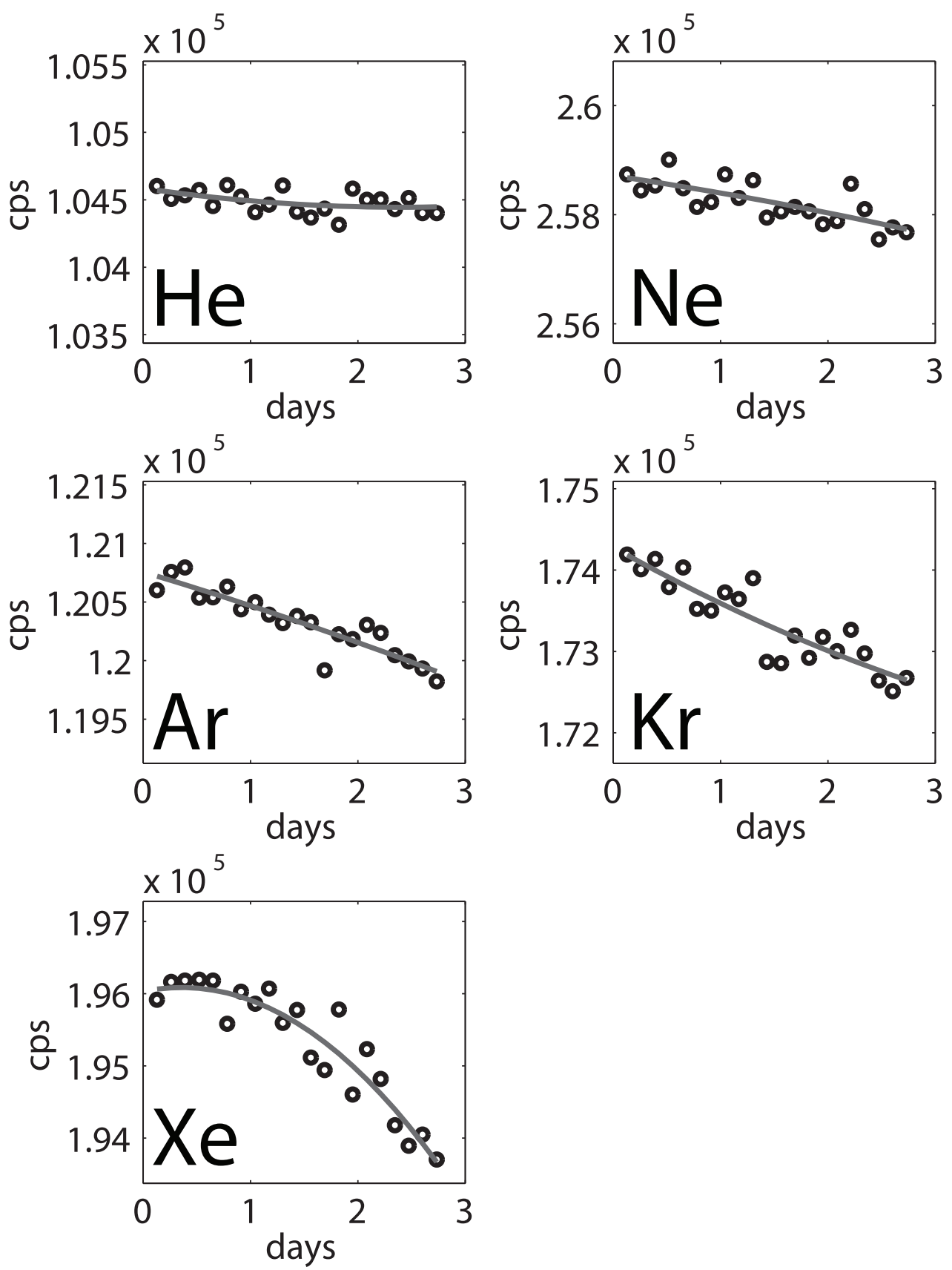
Figure 2-8: Histograms of the deviations (calculated as \%) of the repeated measurements of standards run over several days from a quadratic trend line. The data used for these histograms are the same as depicted in Fig. 2-7. He, $\mathrm{Ne}$ and $\mathrm{Ar}$ have smaller deviations than $\mathrm{Kr}$ or Xe, reflecting that the lighter gases have a smaller variability due to random errors.
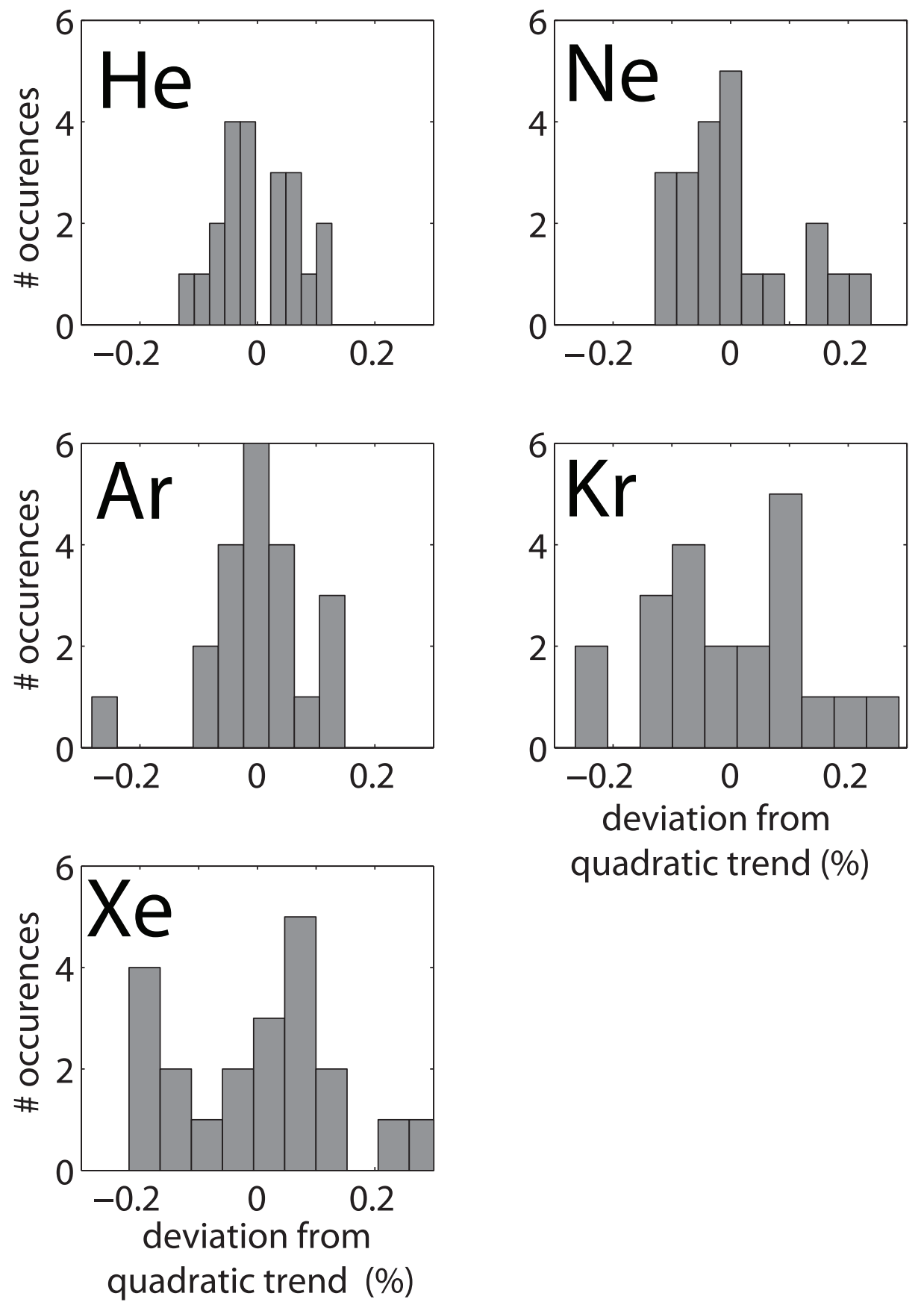
Table 2.8: Performance of the QMS for measuring the major isotopes of the five noble gases in distilled water samples consisting of $90 \mathrm{~g}$ of water equilibrated with laboratory air.

\begin{tabular}{cccccc}
\hline & ${ }^{4} \mathrm{He}$ & ${ }^{20} \mathrm{Ne}$ & ${ }^{40} \mathrm{Ar}$ & ${ }^{84} \mathrm{Kr}$ & ${ }^{132} \mathrm{Xe}$ \\
amount of gas (cm $\left.{ }^{3} \mathrm{STP}\right)$ & $4.12 \times 10^{-6}$ & $1.6 \times 10^{-5}$ & $3.0 \times 10^{-2}$ & $3.9 \times 10^{-6}$ & $2.6 \times 10^{-7}$ \\
\% saturation & $101.1 \%$ & $99.5 \%$ & $99.3 \%$ & $98.5 \%$ & $98.6 \%$ \\
Precision (\%) & $0.8 \%$ & $0.7 \%$ & $0.7 \%$ & $1.0 \%$ & $1.2 \%$ \\
\hline
\end{tabular}

the solubilities of the noble gases depend on the salinity of water, it would be helpful to do the experiment with seawater so as to better be able to interpret our seawater results.

The uncertainties of this method for replicate seawater samples are $0.96 \%$ for $\mathrm{He}, 0.88 \%$ for $\mathrm{Ne}, 0.28 \%$ for Ar, $0.29 \%$ for $\mathrm{Kr}$, and $0.19 \%$ for Xe. These are significantly improved for Ar, $\mathrm{Kr}$ and $\mathrm{Xe}$ but similar for $\mathrm{He}$ and Ne. This may be because (1) our method has improved for $\mathrm{Kr}$ and $\mathrm{Xe}$ since the distilled water test was performed and (2) some of the uncertainty in the saturation anomalies of the heavier gas in the distilled water test was probably a result of temperature inhomogeneities in the water container. The uncertainty has not improved for He and $\mathrm{Ne}$ probably because the uncertainty in those gases is due to bubbles entrained during sample collection. The $\mathrm{He}$ and $\mathrm{Ne}$ results from the distilled water tests can be used to correct all samples for bubble entrainment during sampling.

\subsubsection{Performance of the HIMS for He Isotopes}

The theoretical limit to the precision of the helium isotope measurement is governed by the number of ${ }^{3} \mathrm{He}$ ions collected (Poisson statistics) and that precision is calculated to be $0.06 \%$. The peak shapes are well defined for ${ }^{3} \mathrm{He}$ and ${ }^{4} \mathrm{He}$ with centers of the He peaks at an accelerating voltage of 2844.6 V (Fig. 2-9). There is good separation between ${ }^{3} \mathrm{He}$ and HD (centered at $2837.8 \mathrm{~V}$ ). The valley between the peaks is about $5 \%$ of the ${ }^{3} \mathrm{He}$ peak height. The shape of the HD peak is not symmetric, possibly because of interferences from $\mathrm{H}_{3}$.

A typical running standard has approximately $3.9 \times 10^{-6} \mathrm{cc}$ of He. The signal sizes for the running standard are approximately $1500 \mathrm{cps}$ for ${ }^{3} \mathrm{He}$ as measured on the pulse-counting SEM and $210 \mathrm{pA}$ for ${ }^{4} \mathrm{He}$ 
Figure 2-9: The peak shapes for HD and ${ }^{3} \mathrm{He}$ as measured on a pulse counting secondary electron multiplier (blue) and the peak shape of ${ }^{4} \mathrm{He}$ as measured on the Faraday cup (red) all with 1 second integrations at 0.01 $\mathrm{V}$ resolution. The peaks for the helium isotopes are flat-topped and are centered at $2844.6 \mathrm{~V}$. The peak shape for HD is slightly triangular and is centered at $2837.8 \mathrm{~V}$. There is good separation between the ${ }^{3} \mathrm{He}$ peak and that of HD, with the valley being approximately $5 \%$ of the ${ }^{3} \mathrm{He}$ signal. The SEM background, which is due to ions that have suffered low-angle scattering off resident gas atoms/molecules, is approximately $1 \%$ of the ${ }^{3} \mathrm{He}$ signal and the FC baseline is approximately $0.05 \%$ of the ${ }^{4} \mathrm{He}$ signal.

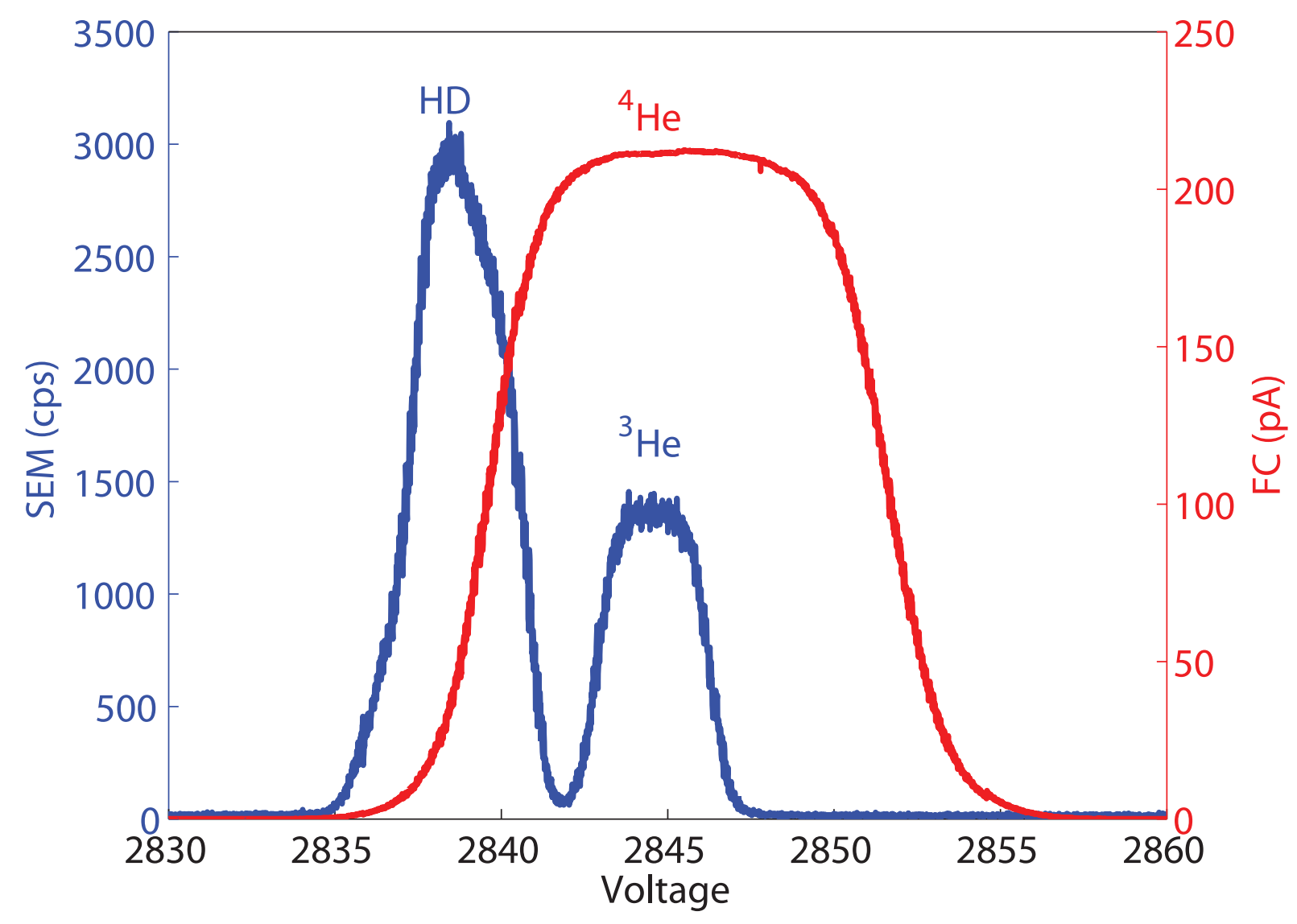


as measured on the Faraday cup with an FET input electrometer. Because of the relatively high partial pressure of He in the HIMS ( 2 to $3 \times 10^{-6}$ torr), there is a background due to ions that have scattered off neutrals. This background is typically $16 \mathrm{cps}$ for a standard or sample, thus resulting in a background that is approximately $1 \%$ of the ${ }^{3} \mathrm{He}$ signal. This background is measured at regular intervals during the analysis by increasing the accelerating voltage to about $2854 \mathrm{~V}$, off but not far from the ${ }^{3} \mathrm{He}$ peak. On the Faraday cup, there is an electrometer DC offset associated with an input bias current in the input JFETs equal to 0.1 $\mathrm{pA}$, which is $0.05 \%$ of the ${ }^{4} \mathrm{He}$ signal and is measured and subtracted for each analysis. Uncertainty in this correction is very small $(\sim 0.01 \%)$.

There are four components to variability in the isotope measurement: (1) variability in the luminosity of the source; (2) variability in the beam steering; (3) variability in the gain of the pulse counting SEM detection system and (4) digitization error (for ${ }^{4} \mathrm{He}$ ) or ion counting statistics (for ${ }^{3} \mathrm{He}$ ). The variability in the luminosity of the source can be determined by repeated measurements on the top of the ${ }^{4} \mathrm{He}$ peak to be $0.068 \mathrm{pA}$, or $0.032 \%$ of a typical standard size. The variability in beam steering can be determined by measuring the current while sitting on the side of the ${ }^{4} \mathrm{He}$ peak to be $0.004 \mathrm{~V}$ in acceleration voltage. The impact of this variability on the measurement depends on peak-shape and slope. A crude calculation, assuming a slope on the top of the peak of 5\%/volt yields an upper bound uncertainty of $0.02 \%$ associated with beam steering variability. The variability in SEM efficiency is an additional source of error; often in mass spectrometers with pulse-counting SEM, the ion to electron conversion efficiency on the entrance to the SEM is less than unity. That is, the SEM may not be detecting all the ${ }^{3} \mathrm{He}$ ions - some ions may not be starting a sufficiently large cascade of electrons for detection of the pulse. The digitization error for ${ }^{4} \mathrm{He}$ is $0.1 \mathrm{pA}$ per second, suggesting a theoretical error of approximately $0.05 \mathrm{pA}$, in close agreement with the observed variability. Ion counting statistics for ${ }^{3} \mathrm{He}$ can be calculated from the number of ions detected (typically of the order $3 \times 10^{6}$ ) to be $0.06 \%$.

The ${ }^{3} \mathrm{He} /{ }^{4} \mathrm{He}$ ratio measured on air standards is $1.0 \times 10^{-6}$. This is calculated by converting the ${ }^{4} \mathrm{He}$ Faraday cup current in pA to ions per second using Coulomb's constant and taking the ratio of the ${ }^{4} \mathrm{He}$ ion count rate to the ${ }^{3} \mathrm{He}$ ion count rate. There is a systematic uncertainty in this conversion, associated with the tolerance of the current sense resistor $\left(10^{10} \mathrm{ohms}, \pm 1 \%\right)$, and the uncertainty of the gain in the electrometer amplifiers $(0.2 \%)$ and in the voltage to frequency converter $(0.1 \%)$. The composite uncertainty is thus $\sim 1 \%$. 
The ratio measured is smaller than the atmospheric ratio of $1.4 \times 10^{-6}$ (Clarke et al., 1976; Hoffman and Nier, 1993). One possible explanation is that there may be mass discrimination in the ion source. We tuned the source on ${ }^{4} \mathrm{He}$ because it is larger and less subject to the ion counting variations seen with ${ }^{3} \mathrm{He}$ and thus perhaps the ${ }^{4} \mathrm{He}$ ions are extracted out of the ion box more easily than the ${ }^{3} \mathrm{He}$ ions. Support for this hypothesis comes from the fact that the apparent isotope ratio $\left({ }^{3} \mathrm{He} /{ }^{4} \mathrm{He}\right)$ increases with time during the analysis.

The estimated efficiency of the ion source is 55\%. This efficiency is calculated from taking the ratio of ions collected in the Faraday cup to the number of ions removed from the system (lost either due to detection or collisions). In a simple experiment, a gas standard was measured continuously on the peak of

${ }^{4} \mathrm{He}$ for 20 minutes. The average current was $209 \mathrm{pA}$. Thus $1.6 \times 10^{12}$ ions were collected. The standard has $3.98 \times 10^{-6} \mathrm{cc} \mathrm{He}=2.05 \times 10^{14}$ atoms of $\mathrm{He}$. When the standard was in the HIMS for 20 minutes, the ${ }^{4} \mathrm{He}$ current decreased by $2.7 \%$. Thus $2.9 \times 10^{12}$ ions are removed from the system. Hence the efficiency, which is equal to ions collected divided by ions removed, is equal to $55 \%$.

\subsection{Discussion}

The method presented here measures all five noble gases from a single sample. Good separation of the noble gases is obtained, even of the heavier noble gases which traditionally have been the most difficult to separate. The results are reproducible and test water samples show reasonable saturation values. In order to achieve these results, we had to resolve several procedural issues. Below we discuss several of the problems that were encountered in developing this method and how we resolved them. Such a discussion may be of use to those who wish to adapt this method for their own analyses.

\subsubsection{Separation of the Noble Gases}

The multiple cryogenic system offers a powerful method for separating the noble gases. Although the principle of the noble gases being released at different temperatures is simple, the actual separation does not necessarily happen in a straight-forward fashion. On a charcoal cryotrap, the release curves for the heavier noble gases overlap significantly, with one gas starting to be released long before the previous gas is $100 \%$ released. Additionally, the heavier gases are released at very high temperatures ( $280 \mathrm{~K}$ for Ar, for 
example) on a charcoal trap, requiring very long cycle times. On a stainless steel cryotrap, these problems are mitigated, as the release curves are sharper and the release temperatures are lower (only $60 \mathrm{~K}$ for $\mathrm{Ar}$, for example). Nonetheless, some small overlap of release curves occurs (Lott, 2001). Furthermore, Ar is orders of magnitude more abundant than $\mathrm{Kr}$ or $\mathrm{Xe}$ in water, making it especially difficult to remove all Ar before analyzing $\mathrm{Kr}$ or Xe fractions.

In order to achieve the best separation of the noble gases, we examined a range of release temperatures and procedures. In general, the cryotrap was operated at its minimum temperature to draw the gases onto it. The temperature is raised to successively higher temperatures to release each noble gas separately. Sometimes it was necessary to compromise on a release temperature, releasing a gas at a temperature below which $100 \%$ of the gas was released in order to prevent releasing too much of the following gas. For example, we found it necessary to release Ar at $60 \mathrm{~K}$, even though only aproximately $80 \%$ of Ar is released at this temperature, in order to prevent $\mathrm{Kr}$ from being released with the Ar. Since sample results are compared to standards, the exact amount of the gas released does not matter, as long as the same percentage is released in every analysis. Nonetheless, the advantage of being near the $100 \%$ release point is that then small fluctuations in the temperature of the cryotrap do not have a large effect on the amount of gas released.

At the optimal release temperatures, we found that large amounts of Ar were being let in with the $\mathrm{Kr}$ and Xe subsamples. Increasing the pumping time at the Ar release temperature did not significantly reduce the amount of Ar being inlet with $\mathrm{Kr}$ and Xe. We successfully reduced the Ar in the $\mathrm{Kr}$ and $\mathrm{Xe}$ inlets by one to two orders of magnitude by isolating, warming, cooling, and pumping on the SSC after the Ar inlet and before starting the $\mathrm{Kr}$ inlet. We hypothesize that some Ar may be initially cryotrapped by the $\mathrm{Kr}$ and $\mathrm{Xe}$ atoms in the SSC. By warming the SSC to $155 \mathrm{~K}$, all the Ar, Kr, and Xe atoms are converted to the gaseous phase. Then when the SSC is slowly cooled to $65 \mathrm{~K}$, the $\mathrm{Kr}$ and Xe recondense but most of the Ar remains in gaseous phase. Pumping on the trap at $62 \mathrm{~K}$ removes the Ar. A second possibility is that the temperature cycling makes it easier to desorb Ar from the surface of the SSC. The Ar is adsorbed to the surface initially. There is a strong kinetic effect with adsorption/desorption processes that perhaps is hastened by temperature cycling, allowing the Ar to be pumped away. In any case, we found that the heating/pumping cycle was necessary to reduce the Ar to less than $20 \%$ of the $\mathrm{Kr}$ signal and less than $1 \%$ of the Xe signal.

Similarly, in order to achieve minimal $\mathrm{Kr}$ in the Xe inlet, we added an additional heating/pumping cycle 
immediately after the $\mathrm{Kr}$ analysis. Again we theorize that perhaps by warming the trap, we convert all the $\mathrm{Xe}$ to gaseous phase, allowing any $\mathrm{Kr}$ cryotrapped by $\mathrm{Xe}$ to be freed. Then after the trap is cooled, $\mathrm{Kr}$ is still in the gaseous form and is pumped away. Or perhaps again it is an absorption/desorption kinetic issue, hastened by the temperature cycle. In any case, by warming the SSC to $155 \mathrm{~K}$, cooling it to $93 \mathrm{~K}$, and pumping, we were able to reduce the $\mathrm{Kr}$ in the $\mathrm{Xe}$ inlet by an order of magnitude. We were then able to make a Xe measurement, in which $\mathrm{Kr}$ accounted for less than $10 \%$ of the Xe signal.

\subsubsection{Matrix Effects of Ar and Xe on $\mathrm{Kr}$}

We observed a "matrix" effect with the SSC in which samples with larger amounts of Ar resulted in smaller $\mathrm{Kr}$ measurements. We hypothesize that this effect occurs because the Ar forms a sorption layer or "ice" at low temperatures that is many layers deep on the trap, rather than forming a simple monolayer. A simple calculation using the area of the trap and the diameter of an Ar atom results in an estimate of order 100 layers of Ar atoms on the trap surface. It is likely that $\mathrm{Kr}$ atoms may be occluded in these layers of Ar. When the $\mathrm{Ar}$ is released from the trap at $60 \mathrm{~K}$, some $\mathrm{Kr}$ is released with it and swept from the trap during head-space expansion of the Ar and pump-out. This leads to a bias where the apparent $\mathrm{Kr}$ composition will appear slightly smaller for a sample with a larger Ar: Kr ratio. We detected no similar effect on Xe, probably because Xe has a much higher release temperature and thus is pulled onto the trap surface at a much higher temperature after the thermal cycling, and hence is layered onto the surface below the Ar layers. Krypton's vulnerability is that it is more similar to Ar in its sorption characteristics and therefore more likely commingles with it.

Our initial procedures resulted in an apparent $\mathrm{Kr}$ "suppression" of about $5 \%$ when doubling the amount of $\mathrm{Ar}$ in the sample. We subsequently modified the procedure to reduce this effect to about $2.5 \%$. The modification that reduced the matrix effect the most was to release $\mathrm{Ar}$ at a lower temperature $(60 \mathrm{~K}$ rather than $62 \mathrm{~K}$ ). This is probably because at the lower temperature, Ar may be less effective as a molecular drag pump, allowing the $\mathrm{Kr}$ to remain sorbed to the trap more easily while Ar is being released. We also changed the temperature cycle of the stainless steel cryotrap before Ar release to only warm the SSC to $80 \mathrm{~K}$, rather than the $155 \mathrm{~K}$ we originally used. We then slowly cool the SSC from $80 \mathrm{~K}$ to $25 \mathrm{~K}$. This strategy is based on the assumption that by raising the SSC to just slightly above Ar release temperature, we convert only Ar 
to the gaseous phase, allowing any $\mathrm{Kr}$ and $\mathrm{Xe}$ that has been trapped within the Ar to settle "to the bottom" of the SSC. Then, as we slowly cool the trap, Ar settles on top of the Kr. This new method presumable reduces (but not eliminates) the Ar-Kr commingling.

Additionally, there is a second matrix effect on $\mathrm{Kr}$ due to $\mathrm{Xe}$. If more $\mathrm{Xe}$ is in a sample, then more $\mathrm{Kr}$ is released from the trap. One possible explanation, is that Xe can cryotrap some $\mathrm{Kr}$ beneath it, preventing the $\mathrm{Kr}$ from being removed when Ar is released at $60 \mathrm{~K}$. A second possibility is that the $\mathrm{Xe}$ atoms may passivate the stainless steel trap, allowing $\mathrm{Kr}$ to be more easily released at the $\mathrm{Kr}$ release temperature. In either case, this effect needs to be accounted for.

Although we were not able to completely eliminate either of these effects, we were able to calibrate them precisely (Fig 2-10). We calibrated these matrix effects by preparing three additional standards composed of pure $\mathrm{Ar}$, pure $\mathrm{Kr}$, and pure $\mathrm{Xe}$. We then ran a number of gas standards with different numbers of additional aliquots of pure Ar, pure $\mathrm{Kr}$, and pure Xe. We performed a multiple nonlinear regression to account for the Ar and Xe effects on the Kr measurements and other Kr "linearity" effects at the same time. We examined a number of functional forms for the correction and found the form that gave the best fit to the data was

$$
R K r_{i}=1+a_{1}\left(R K r c c_{i}-1\right)-a_{2} \Delta A r c c_{i} \cdot R K r c c_{i}+a_{3} \cdot\left(R X e c c_{i}-1\right)
$$

where $i$ refers to quantities of the unknown sample, $a_{1}, a_{2}, a_{3}$ are constants, $R K r_{i}=\frac{K r c p s_{i}}{K r c p s s t d}, R K r c c_{i}=$ $\frac{K r c c_{i}}{K r c c_{s t d}}, R X e c c_{i}=\frac{X e c c_{i}}{X e c c_{s t d}}$, and $\Delta A r c c=A r c c_{i}-A r c c_{s t d}$, with $c c$ referring to amount of gas in cc STP, and $c p s$ referring to the ion count rate measured on the QMS in cps. A non-linear least squares technique was used to determine the optimal coefficients and their uncertainties (Table 2.9). When Eq. 2.1 was applied to the gas standard experiments, the amount of $\mathrm{Kr}$ (in cc STP) calculated matched the known amount to within $0.1 \%$ in most cases, and to $0.2 \%$ if the amount of Ar or Xe were more than $50 \%$ different from the regular standard. The errors in the coefficients and in the measurements were propagated to show that for a $35 \%$ deviation in size between standard and sample, the $\mathrm{Kr}$ correction would yield a $0.14 \%$ uncertainty.

Cold $\left(<15^{\circ} \mathrm{C}\right)$ water samples have relative gas ratios similar to that of a running standard consisting of air and two aliquots of makeup standard. We thus performed similar experiments where we added additional aliquots of pure $\mathrm{Kr}$, pure Ar, and pure Xe to two aliquots of the makeup standard. These coefficients are very similar to those for determined with a reference of a one makeup standard, giving confidence in the 
Figure 2-10: Contour plot of the "matrix effect" of Ar and Xe on the Kr measurements. The relative amount of $\mathrm{Kr}$ is plotted vs. the absolute amount of additional Ar (in std cc) added to the standard and the relative amount of additional Xe (ratio relative to the gas standard and thus unitless). All the standards run in the experiments plotted here had identical amounts of $\mathrm{Kr}$. However, when more Ar (or less Xe) was added, the apparent $\mathrm{Kr}$ ion count rate decreased. Contour lines are drawn at $1 \%$ intervals. White circles reflect the data points from the experiments. Black x show the expected position of seawater samples with respect to a typical running standard comprised of air and the makeup standard. We used the data here as well as additional experiments with pure $\mathrm{Ar}$, pure $\mathrm{Kr}$, and pure Xe standards in order to determine a regression that can correct for these matrix effects. $\mathrm{Kr}$ is the only gas that is affected by a change in Ar or Xe concentrations.

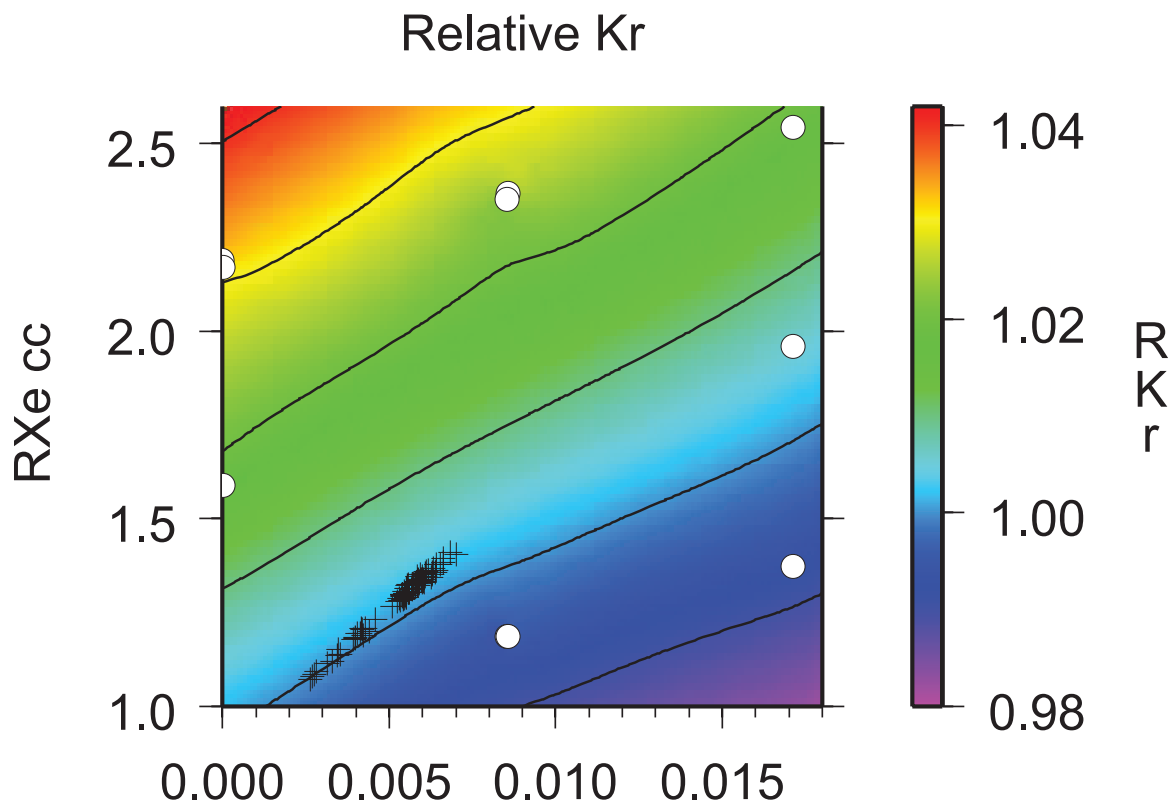

$\Delta \operatorname{Arcc}$

functional form of Eq. 2.1.

\subsubsection{Hydrogen}

To avoid errors associated with preferential ionization and ion collisions associated with other gases, we wanted to only let in one gas at a time into the mass spectrometer. Not only does this necessitate separating all the noble gases, but also it means preventing other gases such as $\mathrm{H}_{2} \mathrm{O}, \mathrm{N}_{2}, \mathrm{O}_{2}, \mathrm{CH}_{4}$, and $\mathrm{H}_{2}$, a number of which are far more abundant than the noble gases, from being inlet into the QMS. The initial getter system used reduced the amount of $\mathrm{H}_{2} \mathrm{O}, \mathrm{O}_{2}$, and $\mathrm{N}_{2}$ to acceptable levels but did not suffice for $\mathrm{H}_{2}$ or $\mathrm{CH}_{4}$. There 
Table 2.9: Coefficients and their uncertainties $(1 \sigma)$ for the matrix effect of $\mathrm{Ar}$ and $\mathrm{Xe}$ on $\mathrm{Kr}$ with respect to a reference standard composed of 1 aliquot of makeup (1MU) gas standard and with respect to a reference standard composed of 2 aliquots of makeup gas standard (2MU).

\begin{tabular}{lccc}
\hline & $a_{1}$ & $a_{2}$ & $a_{3}$ \\
\hline Reference is 1 MU & $1.0692 \pm 0.002$ & $1.2534 \pm 0.09$ & $0.029118 \pm 0.002$ \\
Reference is 2 MU & $1.0454 \pm 0.002$ & $1.2519 \pm 0.04$ & $0.02508 \pm 0.001$ \\
\hline
\end{tabular}

was a large background of $\mathrm{H}_{2}$ in the QMS, with the $\mathrm{H}_{2}$ being released from the stainless steel surfaces in the QMS or processing line or being released by the actuating valves. In order to reduce $\mathrm{H}_{2}$, we added a room temperature Fe-Zr-V getter (SAES ST707) to the QMS chamber. Thus once the sample was inlet, this additional getter sorbed any $\mathrm{H}_{2}$ in the first few seconds of analysis, reducing the $\mathrm{H}_{2}$ by two orders of magnitude. This getter also reduced $\mathrm{N}_{2}$ by one order of magnitude.

For the HIMS, it is especially crucial to reduce the amount of hydrogen since $\mathrm{HD}$ and $\mathrm{H}_{3}$ has a nominal mass of 3 , just as does ${ }^{3} \mathrm{He}$. If hydrogen is too abundant in the spectrometer, the low mass tail of the HD- $\mathrm{H}_{3}$ peak interferes significantly at the ${ }^{3} \mathrm{He}$ mass position. We thus attached three stainless steel containers, each with about $50 \mathrm{~g}$ of getter material (SAES ST707) to the HIMS. We initially baked the HIMS at $300{ }^{\circ} \mathrm{C}$ for 1 week in order to remove hydrogen contained in the stainless steel. When we cooled the HIMS and turned on the ion beam, we found an unacceptable amount of HD (about $6 \times 10^{6} \mathrm{cps}$ ). We thus baked the HIMS again, this time at a higher temperature of $380{ }^{\circ} \mathrm{C}$. Additionally, we first heated the getters to $700{ }^{\circ} \mathrm{C}$ since when the getters are hot, they release their hydrogen load. Then we cooled the getters and kept them at room temperature during the bakeout so that they would sorb the hydrogen released from the ion source and flight tube. We then cooled the flight tube, and only once it was cool did we heat the getters again. By alternating hot getters with hot flight tube we were able to first use the getters as effective pumps for the hydrogen, and then to release the hydrogen directly from the getters into the turbo molecular pump. After this bakeout, the HD was reduced over three orders of magnitude to a value comparable to the ${ }^{3} \mathrm{He}$ ion beam for a typical sample. At this level, the interference was indistinguishable from zero. 


\subsubsection{Methane}

Not only can other gases interfere with ionization inside the QMS, but also the presence of gases other than the noble ones and hydrogen on the cryotrap can change the release characteristics of the noble gases from the SSC. Our original experimentation with another, similarly designed mass spectrometer, showed that when $\mathrm{CH}_{4}$ was drawn onto the SSC, it effectively "activated" the trap surface, which retarded the release of Kr. This is an important potential systematic bias because water samples have approximately a factor of four higher concentration of $\mathrm{CH}_{4}$ than our air standards and thus we observed that our water samples were yielding erroneously low $\mathrm{Kr}$ measurements. Moreover, we were concerned that excessive amounts of $\mathrm{CH}_{4}$, which was released from the SSC at temperatures above $100 \mathrm{~K}$, consequently accompanied the $\mathrm{Kr}$ and $\mathrm{Xe}$ into the QMS, possibly influencing subsequent measurements.

With the aim of reducing $\mathrm{CH}_{4}$, we examined the effect of raising the temperature of the hot half of the flow-through getter. At elevated temperatures (supposedly $>300^{\circ} \mathrm{C}$ ), the getter should crack some of the methane into $\mathrm{C}$ and $\mathrm{H}_{2}$. By raising the temperature from $270^{\circ} \mathrm{C}$ to $310^{\circ} \mathrm{C}$ we decreased the $\mathrm{CH}_{4}$ by $50 \%$. By further increasing the temperature to $350^{\circ} \mathrm{C}$, we made only a modest reduction in the $\mathrm{CH}_{4}$, but more importantly prevented $\mathrm{CH}_{4}$ from gradually increasing over time. Additionally, after drawing the sample onto the cryotraps, we raised the temperature of the cool side of the flow-through getter to $350^{\circ} \mathrm{C}$ and then cooled it again. This temperature cycling resulted in cracking any $\mathrm{CH}_{4}$ that remains in the cool part of the flow-through getter and thus prevented the $\mathrm{CH}_{4}$ from increasing over time. Even with these improvements, however, we still had more $\mathrm{CH}_{4}$ in our water samples than in our standards.

We thus introduced a Pd catalyst (0.47\% Pd on Al Oxide, BASF R0-20/47) into our sample processing system to act on the gas sample prior to gettering. Pd and Pt catalysts on alumina, silica, zirconia, and anatase have been used in the catalytic converter industry to oxidize volatile organic compounds (VOC) including methane (Lyubovsky and Pfefferle, 1998; Janby et al., 2003; Escandon et al., 2005). When the catalyst is at temperatures greater than $450^{\circ} \mathrm{C}$, Pd catalyzes the oxidation of $\mathrm{CH}_{4}$ into $\mathrm{CO}_{2}$ and $\mathrm{H}_{2} \mathrm{O}$. In our system, the catalyst must be placed before the getters since oxgyen is necessary for the oxidation reaction to occur and the getters can then remove the $\mathrm{CO}_{2}$ and $\mathrm{H}_{2} \mathrm{O}$ that are products of the oxidation reaction. Additionally, the catalyst must be placed after the water trap since the catalyst acts like a "sponge," soaking up large quantitites of water vapor and thus is very hard to subsequently pump down. 
After the addition of the catalyst, the amount of $\mathrm{CH}_{4}$ measured in a water sample was equal to the amount measured in an air standard, both being approximately 20,000 cps. Half of this $\mathrm{CH}_{4}$ comes from the processing line and the other half comes from the QMS itself, perhaps produced by reactions of $\mathrm{CO}_{2}$ and the $\mathrm{H}_{2}$ in the stainless steel. Nonetheless, since the amount of $\mathrm{CH}_{4}$ is the same for both standards and samples, if it interferes with our measurements of the noble gases, it would do so the same way for both standards and samples and thus would have no net effect on our analyses.

\subsubsection{Error Analysis}

The total error in the measurement of the noble gases in a sample derives from three main sources. One source is the random and systematic errors associated with the mass spectrometers and processing line when measuring a sample or standard. The second source is the systematic error associated with how well we can calculate the amount of gas in a standard aliquot. The third source is the random or systematic errors introduced in the sample collection or initial extraction of the gases from the water samples. These sources of error and their estimated sizes for the five noble gases as measured on the QMS are listed in Table 2.10.

To estimate the random error associated with the processing of a sample, we ran standards for several days. We then treated half the standards as "unknowns" and used the remaining half of the standards to predict the values of these "unknowns". We used the deviation between the predicted values and the actual values as a measure of the total error in our sample processing. This random error is listed as "error due to repeated measurements of standards" in Table 2.10.

We also considered the error inherent in the QMS measurement of a single sample (called here QMS error). This QMS error is estimated from the uncertainty in the extrapolation of the ion counts back to the end of inlet (Table 2.10). The fact that we are extrapolating back to inlet introduces an error of about 1.5 to 2 times the intrinsic Poisson uncertainty. The extrapolation reduces dependence on any subsequent fractionation or modification within the QMS during analysis. Additionally, the QMS error of a single sample is smaller than the total processing line error (error estimated from repeated standards), suggesting that much of the variability comes from sample processing rather than from the QMS itself. If desired, the QMS error could be minimized further by increasing the number of scans during analysis.

In this method, we calculate the amount of gas in a sample by comparing the ion count measured by 
Table 2.10: Sources of errors in the measurements of the noble gases.

\begin{tabular}{|c|c|c|c|c|c|}
\hline Source of Error & ${ }^{4} \mathrm{He}$ & ${ }^{20} \mathrm{Ne}$ & ${ }^{40} \mathrm{Ar}$ & ${ }^{84} \mathrm{Kr}$ & ${ }^{132} \mathrm{Xe}$ \\
\hline \multicolumn{6}{|l|}{ 1. Air Standards } \\
\hline Repeated Measurements of Standards & $0.10 \%$ & $0.14 \%$ & $0.10 \%$ & $0.14 \%$ & $0.17 \%$ \\
\hline Extrapolation to end of inlet & $0.05 \%$ & $0.10 \%$ & $0.06 \%$ & $0.07 \%$ & $0.06 \%$ \\
\hline Counting Statistics & $0.02 \%$ & $0.05 \%$ & $0.03 \%$ & $0.03 \%$ & $0.02 \%$ \\
\hline Air Standard Calculation & $0.1 \%$ & $0.1 \%$ & $0.1 \%$ & $0.1 \%$ & $0.1 \%$ \\
\hline Temperature & $0.04 \%$ & $0.04 \%$ & $0.04 \%$ & $0.04 \%$ & $0.04 \%$ \\
\hline Relative Humidity & $0.01 \%$ & $0.01 \%$ & $0.01 \%$ & $0.01 \%$ & $0.01 \%$ \\
\hline Pressure & $0.001 \%$ & $0.001 \%$ & $0.001 \%$ & $0.001 \%$ & $0.001 \%$ \\
\hline Aliquot volumes & $0.09 \%$ & $0.09 \%$ & $0.09 \%$ & $0.09 \%$ & $0.09 \%$ \\
\hline Interstitial loss & $0.002 \%$ & $0.002 \%$ & $0.002 \%$ & $0.002 \%$ & $0.002 \%$ \\
\hline Makeup Std & $0 \%$ & $0 \%$ & $0.1 \%$ & $0.13 \%$ & $0.13 \%$ \\
\hline Total error for standards & $0.14 \%$ & $0.17 \%$ & $0.14 \%$ & $0.17 \%$ & $0.20 \%$ \\
\hline \multicolumn{6}{|l|}{ 2. Water Samples } \\
\hline Correction for linearity & $0.007 \%$ & $0.1 \%$ & $0.03 \%$ & - & $0.02 \%$ \\
\hline Correction for matrix effects on $\mathrm{Kr}$ & $0 \%$ & $0 \%$ & $0 \%$ & $0.19 \%$ & $0 \%$ \\
\hline Correction for gas left in bulb & $0.01 \%$ & $0.01 \%$ & $0.03 \%$ & $0.03 \%$ & $0.06 \%$ \\
\hline Total estimated error for samples & $0.14 \%$ & $0.2 \%$ & $0.14 \%$ & $0.25 \%$ & $0.2 \%$ \\
\hline Observed error for duplicate water samples & $0.96 \%$ & $0.88 \%$ & $0.28 \%$ & $0.29 \%$ & $0.19 \%$ \\
\hline
\end{tabular}


the QMS of the sample to the ion count measured by the QMS of an air standard. Thus it is necessary to accurately and precisely calculate how much gas is in an aliquot of air standard. Any error in the calculation of the air standard would lead to a systematic error that would shift all our results. The uncertainties in the calculation of gas in an air standard include the uncertainties in recording the atmospheric conditions (temperature, pressure, relative humidity) when the air standard was collected and the uncertainties associated with determining the volumes of the aliquots and standard cans (Table2.10). In order to minimize the uncertainties in the collection of the air standard, we take several "reference" air standards over the course of months that we compare to our running air standard. The reference air standards and the running air standard agree within $0.2 \%$. We then use these reference standards to better calibrate the running air standard. Additionally, for the cases of $\mathrm{Ar}, \mathrm{Kr}$, and $\mathrm{Xe}$, there is additional error due to the uncertainty in the calibration of the makeup standard.

When measuring water samples, there are potential additional sources of systematic biases due to the differences in the size and composition between our water samples and our air standards. Although the response of the QMS is close to linear, the slope of the linearity calculation has some uncertainty. We could decrease this uncertainty by more determinations of the linearity correction. Additionally, we correct for the effect of $\mathrm{Ar}$ and $\mathrm{Xe}$ on the $\mathrm{Kr}$ measurements and this correction leads to some additional error for our $\mathrm{Kr}$ measurements. Finally, when running water samples, some of the gas is left in the water in the bulb. We correct for this using the weight of the water (measured after each sample has been run) but uncertainties in the weight of the water, in the temperature of the headspace expansion, and in the ratio of the theoretical to observed amount of gas left behind lead to additional uncertainties for water samples. A combination of all the above effects yields an error estimate for our samples (listed as estimated error in Table 2.10).

There is also a source of error associated with the sample collection and initial extraction of the gases. For example, bubbles can stick to the walls of the sample chamber or lurk in the internal geometry of the plug valves on the sample cylinders and thus erroneously increase the $\mathrm{He}$ and $\mathrm{Ne}$ concentrations. Or if the temperature during the extraction is too low, perhaps not all the Xe is extracted from the samples. Thus it is not surprising that the uncertainties as determined by duplicate pairs of water samples are larger than on our gas standards. The truest estimate of the random component of our total measurement and sampling error comes from duplicate samples. The difference between duplicate samples incorporates all the random 
uncertainties in sampling, processing, and measurement. The errors for the duplicates are the highest for He and for $\mathrm{Ne}$, suggesting we may be entraining bubbles during sample collection.

The error on our ${ }^{3} \mathrm{He}$ measurements from the HIMS can be theoretically calculated from Poisson counting statistics to be $0.06 \%$. For an estimate of the actual error observed, we calculated the deviation from a least squares smoothing spline loosely fit to the standards to be $0.05 \%$ to $0.1 \%$. The error estimated this way depends on how tight a fit one uses for the spline. Thus to better assess the error, we used half of the standards to estimate values for the other half and then looked at the deviation for these "unknown" standards. The average deviation was $0.07 \%$, suggesting that our ${ }^{3} \mathrm{He}$ results are close to the theoretical limit. To include the random error from sample collection and processing, we consider the difference between duplicate pairs, which is $0.14 \%$, which is higher than the instrumental error.

\subsection{Conclusions}

We present here a method for measuring all five noble gases from a single water sample. A new feature of this method is incorporation of an automated stainless steel cryogenic trap into the processing line of a QMS. By repeatedly warming, cooling and pumping the trap, we can separate the heavier noble gases, resulting in improved precision. The precision for this method for air standards for $\mathrm{He}, \mathrm{Ne}, \mathrm{Kr}$, and $\mathrm{Xe}$ is good enough to be useful for geochemical estimates of processes such as gas exchange. The method is fully automated and allows measurement of noble gas isotope ratios. The SSC also reduces the time of the measurement, allowing analysis of all five noble gases in approximately three hours. The method here was used on $90 \mathrm{~g}$ water samples but it could also be used on smaller $1 \mathrm{~g}$ samples. Additionally, it could be adapted for use with rock or ice samples.

The variability in our isotope ratios is smaller than in our absolute abundances, suggesting that this method might be improved by using isotope dilution. However, since the error for our water samples is larger than for our standards, the limitations to our measurements does not come from the mass spectroscopy but rather from the initial sample collection and preparation. Thus future studies should include investigation on more reproducible ways to collect and extract the gases from seawater. 


\subsection{Acknowledgments}

I am very grateful to Dempsey Lott for all his time and effort in building the mass spectrometer and for teaching me so much about how to make the best use of it. This work could not have been done without him. I sincerely thank Bill Jenkins for his teaching me how to assess and improve the performance of the mass spectrometer. He gave very helpful advice on potential experiments and great insight on interpreting the results of those experiments. Additionally, I thank Bill for building the electronic controls for the HIMS and for programming the visual basic software used to run MS3. Also special thanks to Burkard Baschek for his help in developing the method for the noble gas line. I wish to thank Mark Kurz for use of the stainless steel cryotrap that I used for the initial testing of this method on the noble gas line. This work was supported by the National Science Foundation Chemical Oceanography program (OCE-0221247), by the Department of Defense (graduate fellowship to RHRS) and by the Woods Hole Oceanographic Institution (postdoctoral fellowship for BB). 


\section{Chapter 3}

\section{Quantifying Seasonal Air-Sea Gas}

\section{Exchange Processes Using Noble Gas}

\section{Time-Series: A Design Experiment}

This chapter appeared as a paper with the same title in Journal of Marine Research:

Rachel H. R. Stanley, William J. Jenkins, Scott C. Doney

Department of Marine Chemistry and Geochemistry,

Woods Hole Oceanographic Institution Woods Hole, Massachusetts, USA

Published in Journal of Marine Research, Vol 64., p. 267-295. (2006) 


\begin{abstract}
A multi-year time-series of measurements of five noble gases ( $\mathrm{He}, \mathrm{Ne}, \mathrm{Ar}, \mathrm{Kr}$, and $\mathrm{Xe}$ ) at a subtropical ocean location may allow quantification of air-sea gas exchange parameters with tighter constraints than currently available by other methods. We have demonstrated this using a one-dimensional upper ocean model forced by 6-hourly NCEP reanalysis winds and heat-flux for the Sargasso Sea near Bermuda. We performed ensemble model runs to characterize the response of the modeled noble gas saturation anomalies to a range of air-sea gas exchange parameters. We then used inverse calculations to quantify the sensitivity of the parameters to hypothetical observations. These calculations show that with currently achievable measurement accuracies, noble gas concentrations in the Sargasso Sea could be used to constrain the magnitude of equilibrium gas exchange to $\pm 11 \%$, the magnitude of the total air injection flux to $\pm 14 \%$, and the magnitude of net photosynthetic oxygen production to $\pm 1.5 \mathrm{~mol} \mathrm{O}_{2} \mathrm{~m}^{-2} \mathrm{y}^{-1}$. Additionally, we can use noble gases to quantify the relative contributions of bubbles that are partially dissolved to bubbles that are completely dissolved. These constraints are based on idealized assumptions and may not fully account for some of the uncertainties in the meteorological data, in lateral transport processes, and in the solubilities of the noble gases. As a limited demonstration, we applied this approach to a time series of $\mathrm{He}, \mathrm{Ne}, \mathrm{Ar}$, and $\mathrm{O}_{2}$ measurements from the Sargasso Sea from 1985 to 1988 (data from Spitzer, 1989). Due to the limited number of gases measured and the lower accuracy of those measurements, the constraints in this example application are weaker than could be achieved with current capabilities.
\end{abstract}




\subsection{Introduction}

Air-sea gas exchange is a crucial component of the biogeochemical cycles of many important gases, including $\mathrm{O}_{2}$ and $\mathrm{CO}_{2}$. However, existing air-sea gas exchange parameterizations have uncertainties of 25\% to 50\% (Liss and Merlivat, 1986; Wanninkhof, 1992; Wanninkhof and McGillis, 1999; Nightingale et al., 2000). Eddy correlation techniques provide estimates based on hourly time scales (Wanninkhof and McGillis, 1999). Radon deficit calculations (Peng et al., 1979) and deliberate dual release experiments (Watson et al., 1991) allow prediction of gas transfer functions for time scales of several days to two weeks. At the opposite end of the spectrum, gas transfer functions determined from natural ${ }^{14} \mathrm{C}$ balance or bomb ${ }^{14} \mathrm{C}$ considerations (Broecker and Peng, 1974; Wanninkhof and McGillis, 1999) are based on decadal or longer time scales. The techniques that determine air-sea gas exchange parameters from direct empirical data, such as purposeful release experiments, are time-consuming and expensive and have only been applied in limited areas of the ocean. Additionally, few of these methods average gas exchange over time-scales of weeks to months, and yet it is those time scales that most affect nutrient cycling and biological production.

A monthly time-series of noble gas measurements may help constrain air-sea gas exchange parameterizations on monthly time-scales and regional spatial scales. Noble gases are an ideal tool for such an investigation because they are chemically and biologically inert, and therefore respond only to physical processes such as air-sea gas exchange, mixing, transport, etc. Seasonal warming and air injection due to large wind events drive noble gases out of equilibrium while diffusive gas exchange works to restore equilibrium. The five stable noble gases, helium (He), neon (Ne), argon (Ar), krypton (Kr), and xenon (Xe), have a wide range of physical properties. The solubilities of the gases differ by a factor of ten and the diffusivities by a factor of five (Wood and Caputi, 1966; Weiss, 1970, 1971; Weiss and Kyser, 1978; Jhne et al., 1987; Hamme and Emerson, 2004). In addition, the solubilities of $\mathrm{Kr}$ and $\mathrm{Xe}$ are strongly dependant on temperature, whereas the solubilities of $\mathrm{He}$ and $\mathrm{Ne}$ are weakly dependant on temperature. This range in properties causes the gases to respond differently to physical forcing and thus allows quantification of a variety of physical processes.

To quantify these physical processes, we must combine noble gas measurements with a numerical model that simulates the noble gas concentrations in a time-evolving environment. The primary objective of the model is to provide a first order, time-varying description of the key factors that influence noble gas distribu- 
tions such as the vertical distribution of temperature, salinity and mixing in the water column. This modeling is easier for two reasons if we study the noble gases near Bermuda in the Sargasso Sea at the Bermuda Atlantic Time-Series Study (BATS) site $\left(31^{\circ} 40^{\prime} N, 64^{\circ} 10^{\prime} \mathrm{W}\right)$. First, a wealth of hydrographic data exists from the BATS site and nearby Station S $\left(32^{\circ} 10^{\prime} \mathrm{N}, 64^{\circ} 30^{\prime} \mathrm{W}\right)$ that can be used to constrain the physical parameters in the model. A biweekly time-series of temperature, salinity, oxygen, and other measurements exists at Station S from 1954 (Schroeder and Stommel, 1969) with a similar monthly time-series at BATS from 1988 (Michaels and Knap, 1996). Second, a one-dimensional vertical model can be used for the gases with fewer problems at this site than at many others. Thermal forcing is one of the largest determinants of gas saturation state, and at the BATS site, the large scale circulation nearly parallels the zero contour of net heat flux (Jenkins and Doney, 2003). Thus the temperature history of the upper water column with respect to thermal forcing does not vary greatly laterally, suggesting that a one-dimensional model may produce reasonable values for noble gas distributions.

In this paper, we present a design experiment: given noble gas measurements at currently achievable measurement accuracy, how well can we quantify air-sea gas exchange processes? First, we describe the model and method used to constrain air-sea gas exchange parameters from noble gas measurements (3.2). Second, we present the choice of physical parameters used in the model and show that the model successfully mimics temperature and salinity data from the Sargasso Sea (3.3). Third, we present a sensitivity study quantifying how well noble gas measurements can constrain a variety of air-sea gas exchange parameters (3.4). Finally, as a limited demonstration we apply this method to a three-year time-series of $\mathrm{He}, \mathrm{Ne}, \mathrm{Ar}$, and $\mathrm{O}_{2}$ measurements collected at Station S from 1985-1988 (Spitzer, 1989) and determine preliminary values for air-sea gas exchange parameters in a subtropical oligotrophic gyre (3.5).

\subsection{Methods}

\subsubsection{Description of the one-dimensional vertical upper ocean model: Physical parameters}

We used a one-dimensional, vertical, modified Price-Weller-Pinkel (PWP) model (Price et al., 1986). The model previously had been extended to include $\mathrm{He}, \mathrm{Ne}, \mathrm{Ar}$, and $\mathrm{O}_{2}$ (Spitzer and Jenkins, 1989), and here we extended it further to include Kr and Xe. Moreover, whereas Spitzer and Jenkins (1989) used climatological 
data to force their model, we used six-hourly National Centers for Environmental Prediction (NCEP) reanalysis heat fluxes, wind stress, precipitation, etc. (Kistler et al., 2001), interpolated for the BATS site. The annually averaged net heat flux obtained from the NCEP reanalysis for this location is negative (net cooling) suggesting that lateral advection balances the heat budget over decadal, or possibly longer, timescales. Thus a positive heat flux, calculated by balancing the sum of the NCEP heat flux terms to zero (on order 30 to 50 $\mathrm{W} \mathrm{m}^{-2}$ ), is added to the model to compensate for the NCEP extraction of heat and to simulate the effects of lateral advection.

In addition, in the model there is an effective heat convergence associated with Ekman pumping. We computed the Ekman pumping from the four day low pass filtered local wind stress curl derived from the NCEP reanalysis data. The Ekman pumping induced vertical velocity is tapered to zero at the bottom of the model domain, in approximate concordance with the Sverdrup relation. The vertical heat flux convergence associated with this flux results in a net heat gain in the model. To prevent the model from warming, a heat flux of order $5 \mathrm{~W} \mathrm{~m}^{-2}$ is removed from the water column. The exact magnitude of this Ekman heat convergence compensation term $(E k m)$ is difficult to calculate explicitly because it depends on the simulated vertical temperature profile in conjunction with the vertical velocity profile, and to a lesser extent on vertical mixing. It thus was treated as a model parameter that was adjusted for long term heat balance. Furthermore, varying Ekm has the effect of partially compensating for errors in the NCEP heat fluxes. We evaluated physical model performance as a function of the magnitude of Ekm, the depth range over which the total heat flux offset (sum of the Ekman compensation and NCEP balance terms) is distributed $(Z)$, the time scale over which the heat budget is forced to balance, and the background vertical diffusivity $\left(K_{z}\right)$.

We determined the optimum values for these tunable physical parameters from ensemble runs $(\mathrm{n} \sim 100)$ calculated using an initially wide range of physical parameter values: $10^{-5}<K_{z}<10^{-4} \mathrm{~m}^{2} \mathrm{~s}^{-1}, 1<$ $E k m<15 \mathrm{~W} \mathrm{~m}^{-2}$, and $10<Z<400 \mathrm{~m}$. We quantitatively compared the output of these runs to BATS and Station S data through the use of a cost function based on the sum of the weighted root mean square difference between model and observations. Quantities included in the cost function were the winter mixed layer depth, summer mixed layer temperature, winter mixed layer temperature, summer mixed layer salinity, average temperature in the top $600 \mathrm{~m}$, and average salinity in the top $600 \mathrm{~m}$. The mixed layer depth contribution to the cost function was weighted by $25 \mathrm{~m}$, the temperatures by $0.5^{\circ} \mathrm{C}$, and the salinities by 0.1 
psu. As a comparison, we also calculated a second cost function based on the root mean square difference between model and observed temperature and salinity at depths ranging from the surface to $150 \mathrm{~m}$. In both cases, a narrower range of physical parameters was then chosen near the optimum and another ensemble run was performed. The process was repeated until the cost functions displayed no significant difference between the optimal values of physical parameters.

The net water flux was determined by the difference between the NCEP precipitation flux and the evaporation, calculated by converting the latent heat flux from $\mathrm{W} \mathrm{m}^{-2}$ to $\mathrm{kg} \mathrm{m}^{2} \mathrm{~s}^{-1}$. The net evaporation minus precipitation is about $65 \mathrm{~cm} \mathrm{y}^{-1}$ so an offset is applied to balance the fresh water flux over the period, assuming that this is typically achieved in the ocean by a lateral freshwater flux divergence. The optimal value for this offset was determined from the BATS salinity data. The model and the gas distributions are not very sensitive to changes in salinity at this location and thus are not sensitive to the value of this offset.

For the sensitivity study (Sec. 3.4), the model was spun up from 1992-1999 with initial conditions of temperature and salinity profiles from BATS data and all gas concentrations at equilibrium. We used the cost function/ensemble run method described above in conjunction with the BATS and Station S data from 1992 to 2002 in order to calculate the optimal physical parameters for this time period. Then simulated noble gas concentrations from 1999-2002 were used to quantitatively investigate potential constraints on air-sea gas exchange parameters.

For the example application (Sec. 3.5), the model was spun up from 1978 to 1985 and then simulations from 1985 to 1988 were used in conjunction with time-series data to determine the air-sea gas exchange parameters. To determine the optimal physical parameters, we used an inverse analysis with BATS temperature and noble gas data. To determine a reasonable range of physical parameters, we used the cost function/ensemble run method described above. This range of physical parameters allowed us to calculate the additional uncertainty in the air-sea gas exchange parameters due to uncertainty in the physical parameters. The optimal physical parameters for the 1992-2002 decade differed from those for the 1978-1988 time period, probably because of differences in lateral processes (such as eddies) that are not resolved in the model. 


\subsubsection{Description of the model: Gas exchange parameters}

Gas exchange processes are modeled according to standard literature parameterizations with adjustment permitted through the use of tunable model parameters. The total flux of a gas, $F_{T O T}$, is the sum of the flux due to diffusive gas exchange, $F_{G E}$, and the flux due to air injection, $F_{A I}$ :

$$
F_{T O T}=F_{G E}+F_{A I}
$$

The diffusive gas exchange flux, $F_{G E}\left(\mathrm{~mol} \mathrm{~m}^{-2} \mathrm{~s}^{-1}\right)$ is given by

$$
F_{G E}=\gamma_{G} \cdot 7.9 \times 10^{-8} \frac{S c}{660}^{-0.5} u_{10}^{n}\left(C_{i, e q}-C_{i, w}\right)
$$

where $\gamma_{G}$ is a tunable model parameter of order 1 controlling the magnitude of the flux, the coefficient is the constant used by Wanninkhof and McGillis (1999) converted to units of $\mathrm{m} \mathrm{s}^{-1}, S c$ is the Schmidt number (ratio of the kinematic viscosity to the molecular diffusivity of the gas of interest), $u_{10}$ is the wind speed $\left(\mathrm{m} \mathrm{s}^{-1}\right)$ at $10 \mathrm{~m}$ height above sea-surface, $n$ is the exponent controlling the wind-speed dependency of the flux, and $C_{i, w}$ and $C_{i, e q}$ are the concentrations of gas $i$ in the water and at equilibrium, respectively. A cubic wind speed dependency ( $n=3$ ), as proposed by Wanninkhof and McGillis (1999), is used in most model runs. In the few runs with a quadratic wind speed dependency $(n=2)$, the value of the coefficient changes to $8.6 \times 10^{-7} \mathrm{~m} \mathrm{~s}^{-1}$, in order to scale the total gas flux to the global average ${ }^{14} \mathrm{C}$ exchange rate (for details see Wanninkhof, 1992).

In the model, the flux due to air injection, $F_{A I}$, has contributions from two types of bubbles. Some bubbles are completely trapped, i.e. completely dissolved after injection, and therefore inject air of atmospheric abundances into the water column. Other bubbles only partially dissolve before rising to the surface, in which case the injected gases are fractionated as a function of diffusivity and solubility (Fuchs et al., 1987; Jenkins, 1988a; Keeling, 1993; Woolf, 1993). Thus the total air injection flux, $F_{A I}$, is equal to

$$
F_{A I}=F_{C}+F_{P}
$$

where $F_{C}$ refers to the flux from bubbles that are completely trapped and $F_{P}$ refers to the flux from bubbles 
that are only partially dissolved.

The flux due to completely trapped bubbles is dependent on the partial pressure of the gas in the atmosphere and the volume entrainment rate of the air entrained. This volume entrainment rate is given by a combination of the whitecap coverage formulation of Monahan and Torgersen (1990) with the air entrainment velocity estimate of Keeling (1993):

$$
F_{C}=\gamma_{A c} \cdot A_{c} \cdot 2 \times 10^{-6}\left(u_{10}-2.27\right)^{3} \nu_{a} \frac{P_{i, a}}{R T}
$$

where $\gamma_{A c}$ is a tunable model parameter of order 1 that scales the magnitude of the complete trapping flux, $A_{c}$ is a constant coefficient equal to $1.4 \times 10^{-3}, \nu_{a}$ is the air entrainment velocity $\left(0.01 \mathrm{~m} \mathrm{~s}^{-1}\right), R$ is the gas constant $\left(8.31 \mathrm{~J} \mathrm{~mol}^{-1} \mathrm{~K}^{-1}\right), T$ is the temperature $(\mathrm{K})$, and $P_{i, a}$ is the partial pressure of gas $i$ in the atmosphere $(\mathrm{Pa})$. We calculated the coefficient $A_{c}$ by performing ensemble runs $(\mathrm{n} \sim 100)$ and then using a cost function to determine the value of Ac that allowed the best match to $\mathrm{He}, \mathrm{Ne}$, and Ar data from the Sargasso Sea. Eq. 3.4 is valid for $u_{10} \geq 2.27 \mathrm{~m} \mathrm{~s}^{-1}$. At $u_{10}<2.27 \mathrm{~m} \mathrm{~s}^{-1}, F_{c}$ is set to 0 .

The partial bubble trapping flux depends upon the volume entrainment rate, the solubility and diffusivity of the gas, and the difference between the partial pressure of the gas in the bubble and in the water :

$$
F_{P}=\gamma_{A p} \cdot A_{p} \cdot 2 \times 10^{-6}\left(u_{10}-2.27\right)^{3} \nu_{a} \alpha_{i}^{a} D_{i}^{b} \frac{P_{i, b}-P_{i, w}}{R T}
$$

where $\gamma_{A_{p}}$ is a tunable model parameter of order 1 that scales the magnitude of the partial trapping flux, $A_{p}$ is a constant coefficient equal to $2 \times 10^{5}, \alpha$ is the Bunsen solubility coefficient of gas $i, D$ is the diffusivity $\left(\mathrm{m}^{2} \mathrm{~s}^{-1}\right.$ ) of gas $i, a$ and $b$ are exponents controlling the power dependency of $\alpha$ and $D$ respectively, and $P_{i, b}$ and $P_{i, w}$ are the partial pressures of the gas in the bubble and in the water $(\mathrm{Pa})$. We calculated the coefficient $A_{p}$ by performing ensemble runs $(\mathrm{n} \sim 100)$ and then using a cost function to determine the value of $A_{p}$ that allowed the best match to existing $\mathrm{He}, \mathrm{Ne}$, and Ar data from the Sargasso Sea. The power dependency of $\alpha$ and $D$ can be determined theoretically depending on whether the bubbles are believed to be clean $(a=1$, $b=1 / 2)$ or dirty ( $a=1, b=2 / 3$ ) (Levich, 1962) or can be determined by modeling studies of characteristic bubble populations ( $a=0.7, b=0.35$ ) (Keeling, 1993). In this study, we examined the sensitivity of the results to the choice of $a$ and $b$. Unless noted otherwise, we will report results for $a=1, b=2 / 3$. Eq. 3.5 
is valid only for $u_{10}<2.27 \mathrm{~m} \mathrm{~s}^{-1}$. At $u_{10}<2.27 \mathrm{~m} \mathrm{~s}^{-1}, F_{p}$ is set to 0 . Eq. 3.5 is similar to the partial trapping flux equation used by Keeling (1993) when appropriate scaling factors and units are used.

The partial pressure of the gas in the bubble, $P_{i, b}$, depends on the size and depth distribution of the bubbles and thus is a complicated problem of bubble dynamics. Here, however, $P_{i, b}$ is simply approximated by:

$$
P_{i, b}=X_{i}\left(P_{a t m}+\rho g h_{b u b}\right)
$$

where $X_{i}$ is the mole fraction of gas $i$ in dry air, $P_{a t m}$ is the atmospheric pressure of dry air $(\mathrm{Pa}), \rho$ is the density of water $\left(\mathrm{kg} \mathrm{m}^{-3}\right), g$ is the gravitational acceleration $\left(9.81 \mathrm{~m} \mathrm{~s}^{-1}\right)$ and $h_{b u b}(\mathrm{~m})$ is the average dissolution depth for the bubbles. This expression takes into account the hydrostatic pressure felt by the bubble but neglects the effects of surface tension on the bubble. The depth $h_{b u b}$, used for the hydrostatic pressure calculation, is approximated by:

$$
h_{b u b}=\frac{1}{2}\left(0.3 u_{10}-1.1\right)
$$

which is the bubble cloud residence depth/wind speed relationship of Graham et al. (2004), multiplied by a bubble depth scaling factor of $\frac{1}{2}$ to take into account that the bubbles partially dissolve along their entire downward and upward transect rather than simply at their residence depth. The results were not sensitive to the choice of bubble depth scaling factor since a decrease in the scaling factor could be compensated for by an increase in $\gamma_{A p}$. Eq. 3.7 is valid only for $u_{10} \geq 3.7 \mathrm{~m} \mathrm{~s}^{-1}$. At $u_{10}<3.7 \mathrm{~m} \mathrm{~s}^{-1}, h_{b u b}$ is set to 0 .

Both the diffusive gas exchange flux and the air injection flux parameterizations (Eq. 3.2 and Eq. 3.5) require knowledge of the solubilities of the noble gases. These solubilities were determined by Weiss in the 1970s (Weiss, 1970, 1971; Weiss and Kyser, 1978). More recently, the solubilities of Ne and Ar were redetermined by Hamme and Emerson (2004b), with these newer results differing from those of Weiss by $1 \%$ for $\mathrm{Ne}$ and $0.4 \%$ for Ar. We performed most of the calculations with the $\mathrm{Ne}$ and Ar solubilities of Hamme and Emerson. We assumed that the difference in the Ne solubilities between Weiss and Hamme and Emerson was due to a systematic difference in technique. We thus scaled the Weiss solubility values for $\mathrm{He}$ (which was not determined by Hamme and Emerson) with the same correction factor that was needed to 
make the Weiss Ne solubility equal to the Hamme and Emerson Ne solubility. This correction factor, which depends on temperature, ranges from $0.5 \%$ at $18^{\circ} \mathrm{C}$ to $1.5 \%$ at $29^{\circ} \mathrm{C}$. We performed some calculations with the Weiss solubilities in order to assess the effect of different solubility functions. Additionally, we propagated the uncertainty in solubility determination, when appropriate, throughout all the calculations.

Biological production is incorporated through the use of an idealized oxygen production and consumption profile. In the upper $100 \mathrm{~m}$, the oxygen productivity profile, Prod, is represented by a sine curve according to

$$
\operatorname{Prod} \propto \sin \frac{\pi z}{100} \quad \text { for } \quad z<100 \mathrm{~m}
$$

where $z$ is depth (m). Below $100 \mathrm{~m}$, oxygen is consumed exponentially:

$$
\operatorname{Prod} \propto \exp \left(\frac{-(z-100)}{z^{*}}\right) \quad \text { for } \quad z>100 \mathrm{~m}
$$

where $z^{*}$ is a scaling depth of $295 \mathrm{~m}$ that was chosen based on tritium-helium dating (Jenkins, 1980). Since oxygen production by photosynthesis above $100 \mathrm{~m}$ should approximately balance oxygen consumption from remineralization below $100 \mathrm{~m}$, the integrated production above $100 \mathrm{~m}$ was scaled to balance the integrated production below $100 \mathrm{~m}$. The total oxygen production and consumption, $P_{T O T}$, is then scaled by tunable model parameter $\gamma_{P}$ and seasonally modulated with maximum production in early spring (Musgrave et al., 1988; Spitzer and Jenkins, 1989), according to

$$
P_{\text {TOT }}=\gamma_{P} \cdot \operatorname{Prod} \cdot\left[1+\sin \left(2 \pi\left(t-t_{o}\right)\right)\right]
$$

where $t$ is the model time and $t_{o}$ is 1995 (a year in the middle of the time-series). This is an idealized approach to biological productivity and at the moment is not intended to give new information about biological productivity at the BATS site. Rather it simply illustrates that $\mathrm{O}_{2}$ and $\mathrm{Ar}$ measurements can be used to constrain biological productivity. Future work will likely use better data assimilation techniques for oxygen and argon in order to estimate useful values of biological production at the BATS site. 


\subsubsection{Linearization and inverse technique}

The equations above mathematically describe the effect of diffusive gas exchange and air injection on the signatures of the noble gases. Qualitatively, the equations illustrate that there are three types of forcing for the noble gases: thermal forcing which changes the saturation state of the gas, diffusive gas exchange, and air injection. For insoluble gases such as $\mathrm{He}$ and $\mathrm{Ne}$, the diffusive gas exchange flux balances the air injection flux, making separation of the terms difficult. For soluble gases such as $\mathrm{Kr}$ and $\mathrm{Xe}$, the air injection flux is relatively less important. Thus thermal heating (which is directly observed) causes a diffusive gas exchange flux, which can then be calculated from mass budgets. We then used this estimated diffusive gas exchange flux and the $\mathrm{He}$ and $\mathrm{Ne}$ results to quantify the air injection fluxes. In practice, we actually determined the diffusive and air injection fluxes simultaneously using all five noble gases, as described below.

The four tunable geochemical model parameters in the above equations are gas exchange magnitude $\gamma_{G}$, complete bubble trapping magnitude $\gamma_{A c}$, partial bubble trapping magnitude $\gamma_{A p}$, and biological production magnitude $\gamma_{P}$. In order to quantify these factors, we first constructed a reference case by running the model using the most probable values of all model parameters (Table 3.1). For $\gamma_{G}$ and $\gamma_{P}$, literature values were used to assign the reference case. For $\gamma_{A c}$ and $\gamma_{A p}$, reference values of 1 were chosen since the coefficients $A_{c}$ and $A_{p}$ had been calculated to best match observations. Second, we ran the model with a range of values for a given model parameter, holding all other model parameters constant at the reference values, in order to linearize the response of an observational metric to a given model parameter. The observational metrics are measurable quantities, such as the surface saturation anomaly of a noble gas. This linearization ultimately allowed us to estimate how well noble gas measurements, at current measurement capabilities, can constrain air-sea gas exchange parameters. The linearizations are robust to data noise, and physical model error and they provide direct interpretation of parameter impact on observational metrics and error propagation. The sensitivity study shows that the approximation of a linear response is appropriate.

As an initial step, we used the linearizations in a partial derivative method to determine which observational metrics offer the best constraints on a given model parameter. This approach assumes all other model parameters are known with zero error. All uncertainties in this paper refer to $1 \sigma$ values. The estimated uncertainty in the model parameter, $\sigma_{\text {param }}$, which is essentially the constraint on the model parameter, can be calculated from the following equation: 
Table 3.1: Reference case values for the tunable gas exchange model parameters chosen either according to literature values $\left(\gamma_{G}, \gamma_{P}\right)$ or to best match previous observations $\left(\gamma_{A c}, \gamma_{A p}\right)$. All of the parameters are unitless except for the oxygen production parameter $\gamma_{P}$.

\begin{tabular}{ccc}
\hline Model & Parameter & Description Reference Value \\
\hline$\gamma_{G}$ & Magnitude of diffusive gas exchange & 1 \\
$\gamma_{A c}$ & Magnitude of complete trapping component of air injection & 1 \\
$\gamma_{A p}$ & Magnitude of partial trapping component of air injection & 1 \\
$\gamma_{P}$ & Magnitude of net biological production & $5 \mathrm{~mol} \mathrm{O}_{2} \mathrm{~m}^{-2} \mathrm{y}^{-1}$ \\
\hline
\end{tabular}

$$
\frac{\sigma_{\text {metric }}}{\sigma_{\text {param }}}=\frac{\Delta \text { ObservationalMetric }}{\Delta \text { ModelParameter }}=\text { slope }
$$

where $\sigma_{m e t r i c}$ is the estimated uncertainty in the observational metrics achievable with present measurement capabilities and slope refers to the slope calculated from the linearization. Laboratory experiments indicate that it is possible to achieve at least $0.1 \%$ measurement uncertainty on measurements of $\mathrm{He}, \mathrm{Ne}$, and $\mathrm{Ar}$ and at least $0.2 \%$ measurement uncertainty on measurements of Xe using peak height manometry on a quadrupole mass spectrometer. Such uncertainties depend on adequate purification and separation of gases to eliminate matrix effects, and highly reproducible experimental procedures achieved by precise computer control of all processing. There will be additional error due to sampling and gas extraction. However, systematic biases can be assessed by careful experimentation, and random errors can be overcome by oversampling the water column (e.g., multiple samples within the mixed layer).

The situation is more complicated than the partial derivative method suggests because we do not know, a priori, the values for any of the model parameters and because the metrics depend on more than one parameter. In order to take into account this complexity, we also calculated the parameter constraints using a full inverse method that utilizes multiple observational metrics. For the full inverse method, we used simulated "data" of the distributions of the five noble gases from a model run with reference model parameters. Then we used singular value decomposition (SVD) and the linearizations to simultaneously determine all of the model parameters from the "data" weighted by the currently achievable measurement uncertainty of 
the observational metrics. The uncertainties determined from the SVD calculations give us the potential constraints on the model parameters. In the future, when we have an actual dataset of all five noble gases, we will explore using nonlinear optimization techniques rather than the SVD.

Additionally, we calculated linearizations using different sets of physical parameters (see Section 3.3 for description of the sets of physical parameters) and thus determined the uncertainty produced by ambiguity in the one-dimensional model physics. We added in quadrature the uncertainty due to the noble gas measurements as determined from the SVD and the uncertainties due to model physics to determine final constraints on the model parameters.

\subsection{Choice of physical parameters}

The physical model is able to mimic reasonably well the mixed layer depth, temperature, salinity, and average temperature in the top $600 \mathrm{~m}$ (approximately proportional to total heat content) for a ten-year period when the appropriate physical parameters are chosen (Fig. 3-1). The root mean square deviations between model and data are $70 \mathrm{~m}$ for mixed layer depth, $1.2^{\circ} \mathrm{C}$ for mixed layer temperature, $0.13 \mathrm{psu}$ for mixed layer salinity, and $0.4^{\circ} \mathrm{C}$ for average temperature in the top $600 \mathrm{~m}$. The winter mixed layer depths predicted by the model have the largest root mean square deviation from the data relative to the weights assigned in the cost function. This may be because the mixed layer depth is sensitive to convection of relatively homogeneous mode waters, and thus small year to year variations in lateral heat transport, mesoscale eddies, and errors in heat fluxes can lead to large variations in winter mixed layer depth (Doney, 1996). No long-term drift is apparent in the optimized model, even when the model is run for forty-five years. The model-data agreement suggests that the model is an adequate description for the seasonal physical evolution used for the noble gases.

The best set of physical parameters for the time period 1992 to 2002 consist of $K_{z}=6 \times 10^{-5} \mathrm{~m}^{2} \mathrm{~s}^{-1}, Z=$ $100 \mathrm{~m}$, and $E k m=6.5 \mathrm{~W} \mathrm{~m}^{-2}$. However, we found that the parameters work in concert, with various sets of physical parameters yielding similar cost functions. If $K_{z}$ ranged from $2 \times 10^{-5} \mathrm{~m}^{2} \mathrm{~s}^{-1}$ to $8 \times 10^{-5} \mathrm{~m}^{2} \mathrm{~s}^{-1}$, we could still achieve reasonably low cost functions. For model runs with $K_{z}$ outside this range, the cost functions increased significantly and the model results could no longer match the seasonal temperature cycle observed at BATS. We thus carried out the modeling and determination of air-gas exchange parameters with 
Figure 3-1: Comparison of model predictions (-) with BATS/Station S data (o) for mixed layer depth, mixed layer temperature, average temperature in the top $600 \mathrm{~m}$, and mixed layer salinity. Mixed layer depth was determined from BATS/Station $\mathrm{S}$ data using a potential density criterion of $\Delta 0.1 \mathrm{~kg} \mathrm{~m}^{-3}$. Physical parameters used in this reference simulation are background vertical diffusion $K_{z}=6 \times 10^{-5} \mathrm{~m}^{2} \mathrm{~s}^{-1}$, Ekman heat convergence $E k m=6.5 \mathrm{~W} \mathrm{~m}^{-2}$, and depth to which heat offset is added $Z=100 \mathrm{~m}$.
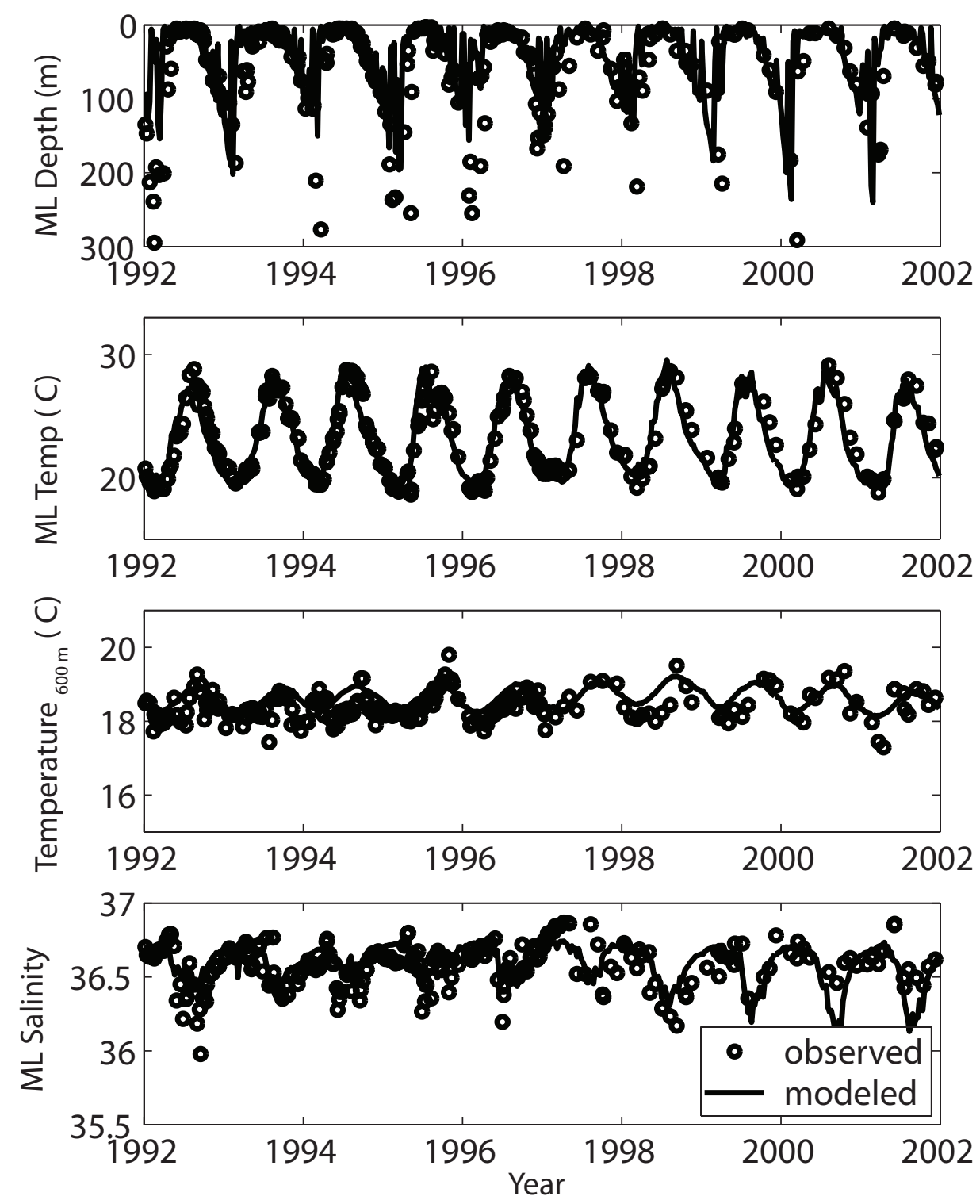
three sets of physical parameters: (1) the optimal set, as listed above (all results in figures and tables are for this case); (2) $K_{z}=8 \times 10^{-5} \mathrm{~m}^{2} \mathrm{~s}^{-1}, E k m=5 \mathrm{~W} \mathrm{~m}^{-2}, Z=100 \mathrm{~m}$; and (3) $K_{z}=2 \times 10^{-5} \mathrm{~m}^{2} \mathrm{~s}^{-1}, E k m=10 \mathrm{~W}$ $\mathrm{m}^{-2}, Z=100 \mathrm{~m}$. We then used the difference in the calculated air-sea gas exchange parameter values as a first-order approximation for the uncertainty due to choice of physical parameters. The choice of physical parameters differs slightly from decade to decade, perhaps because of differences in lateral processes (such as eddies) that are not resolved in the model, or trends in unresolved biases in the NCEP data. For the time period 1978 to 1989 , we also performed calculations with three sets of physical parameters: (1) the best physical parameters $K_{z}=3.6 \times 10^{-5} \mathrm{~m}^{2} \mathrm{~s}^{-1}, Z=100 \mathrm{~m}$, and $E k m=5.5 \mathrm{~W} \mathrm{~m}^{-2}$; (2) $K_{z}=8 \times 10^{-5} \mathrm{~m}^{2} \mathrm{~s}^{-1}$, $E k m=5 \mathrm{~W} \mathrm{~m}^{-2}, Z=100 \mathrm{~m}$; and (3) $K_{z}=2 \times 10^{-5} \mathrm{~m}^{2} \mathrm{~s}^{-1}, E k m=10 \mathrm{~W} \mathrm{~m}^{-2}, Z=100 \mathrm{~m}$.

\subsection{Sensitivity Study: Constraints on air-sea gas exchange parameters from the noble gases}

\subsubsection{Model results: Noble gas behavior}

The model shows that the individual noble gases have different signatures in the mixed layer (Fig. 3-2) and the upper ocean (Fig. 3-3) because the noble gases have a range of physical properties. We measure and model concentrations of the noble gases. However, to ease interpretation, all noble gas results are presented here as saturation anomalies, $\Delta$, which are defined as

$$
\Delta=\left(\frac{C_{i, w}}{C_{i, e q}}-1\right) \times 100
$$

where $C_{i, w}$ is the concentration of gas $i$ in the water and $C_{i, e q}$ is the concentration of gas $i$ at equilib-

rium. Positive (negative) saturation anomalies reflect that the gas is supersaturated (undersaturated), and the magnitude of the saturation anomalies corresponds to the magnitude of the departure from equilibrium. Although a similar pattern of saturation anomalies is seen from one year to another, the exact magnitude of the saturation anomalies differ each year because the saturation anomalies are driven by physical processes, such as wind forcing, mixed layer depth, and mixed layer temperature, which also differ each year.

Helium and Ne are relatively insoluble, with a weak solubility dependence on temperature (Weiss, 1971; 
Figure 3-2: Surface (mixed layer) saturation anomalies $\left(\Delta_{\text {surface }}\right)$ for the five noble gases and oxygen as predicted by the model using reference case model parameters. Note that $\mathrm{He}$ and $\mathrm{Ne}$ form one group with $\Delta \mathrm{He}$ at a maximum in the winter, whereas $\mathrm{Ar}, \mathrm{Kr}, \mathrm{Xe}$, and $\mathrm{O}_{2}$ form a second group and are at a maximum in the summer. Furthermore, this second group of gases has a much larger range in surface saturation anomalies.

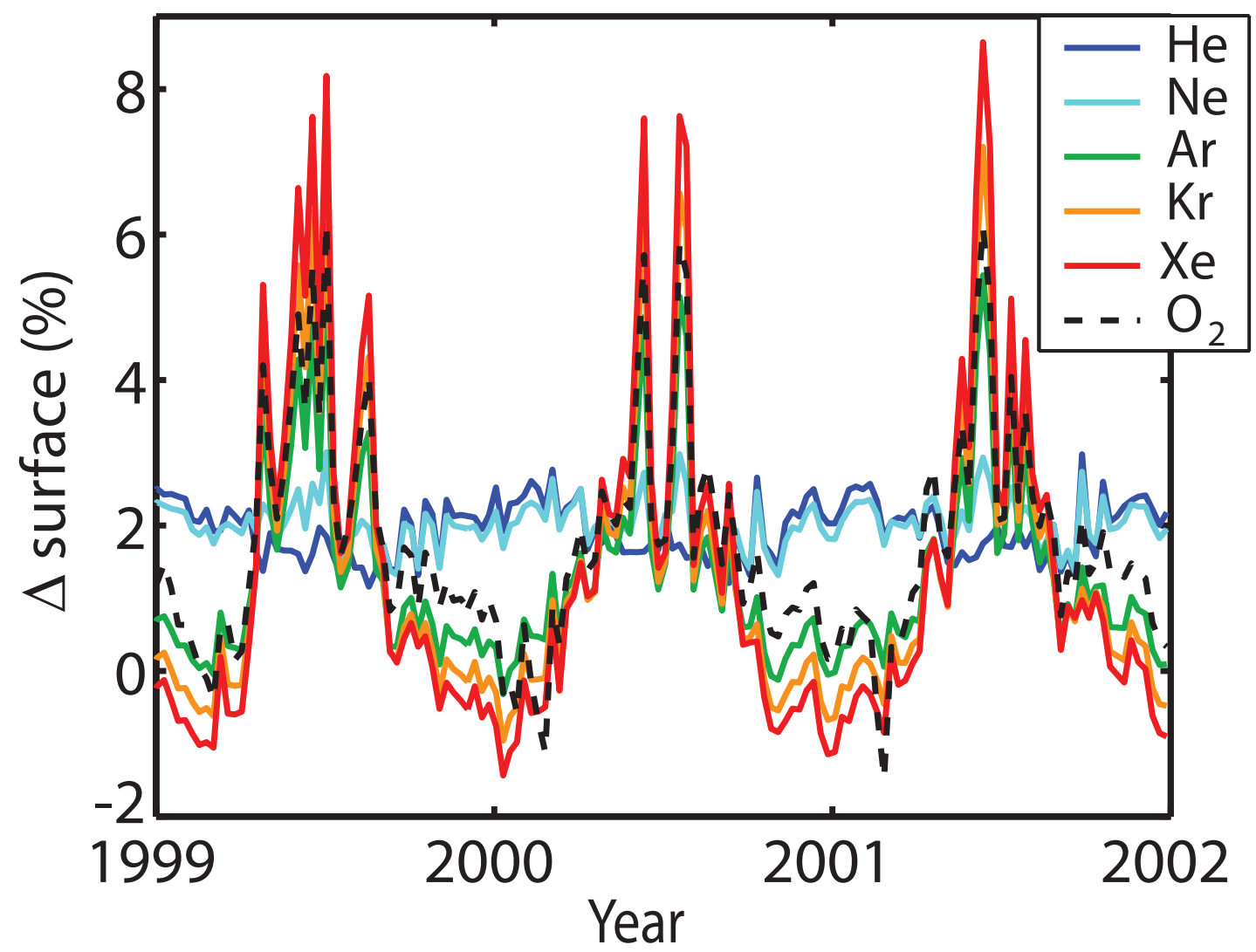


Figure 3-3: Saturation anomalies for all five noble gases in the upper $300 \mathrm{~m}$ of the water column as predicted by the model using reference case model parameters. Contours are drawn at $0.5 \%$ spacing for $\mathrm{He}$ and $\mathrm{Ne}$ and at $2 \%$ spacing for $\mathrm{Ar}, \mathrm{Kr}$, and $\mathrm{Xe}$. The range in Xe saturation anomalies is four times that of He saturation anomalies.
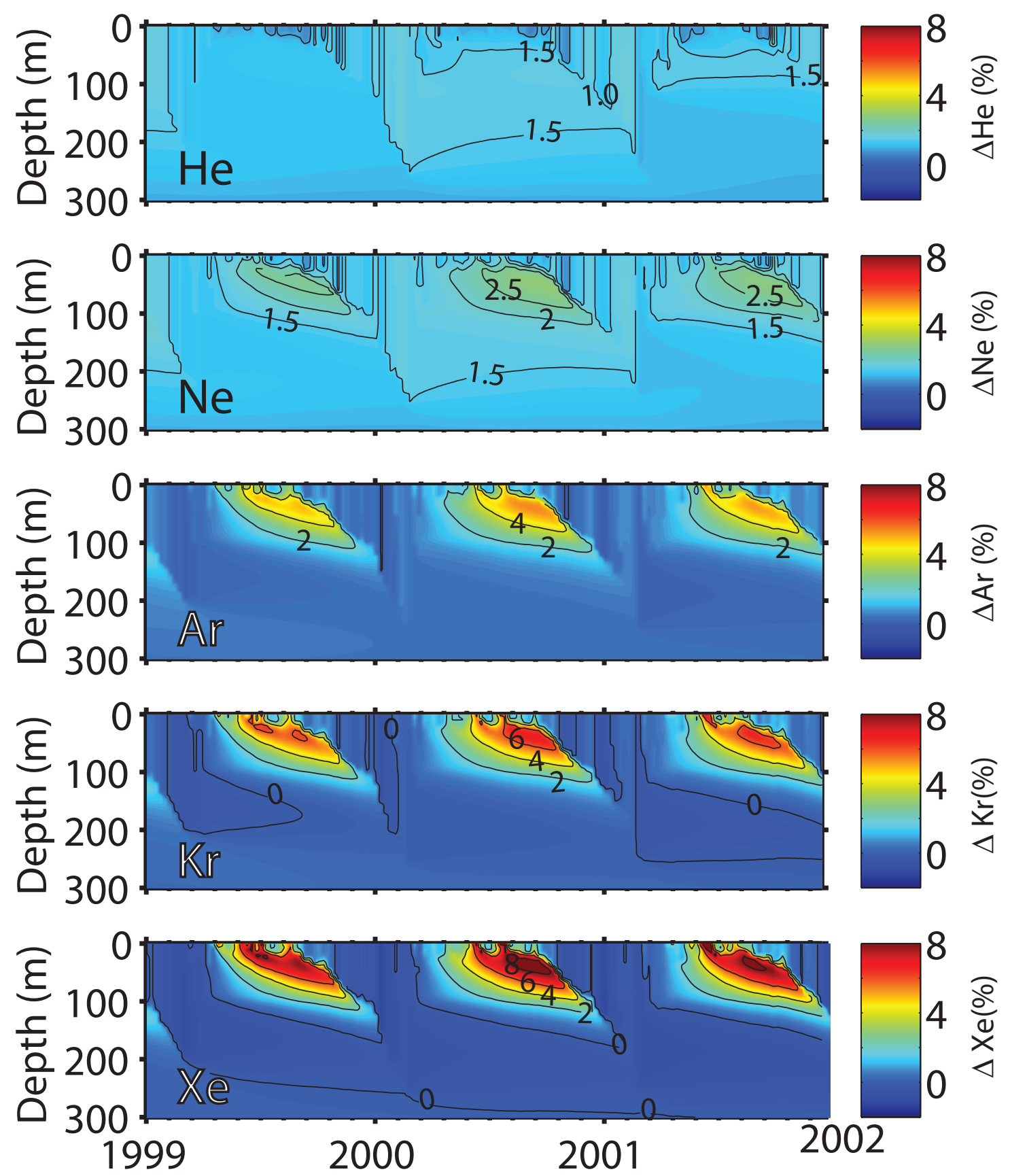
Hamme and Emerson, 2004b). Helium and Ne are thus sensitive to both air injection processes and to diffusive gas exchange. The model shows the maximum surface saturation anomalies of $\mathrm{He}$ are in the winter, when the winds are strongest causing large air injection events. The surface saturation anomalies of He and $\mathrm{Ne}$ are thus good choices for observational metrics for air injection magnitude. The difference between the winter and summer surface saturation anomalies, the seasonal amplitude, is used as the observational metric in order to minimize the effect of any systematic error in the solubility functions of the gases. Additionally, since $\mathrm{He}$ and $\mathrm{Ne}$ have similar solubilities but different diffusivities, the ratio between $\mathrm{He}$ and Ne surface saturation anomalies is a useful observational metric for diffusively mediated processes, such as the magnitude of partially trapped bubbles.

In contrast, $\mathrm{Kr}$ and $\mathrm{Xe}$ are more soluble with a strong solubility dependence on temperature (Wood and Caputi, 1966; Weiss and Kyser, 1978), and thus $\mathrm{Kr}$ and Xe respond primarily to diffusive gas exchange. Saturation anomalies of $\mathrm{Kr}$ and $\mathrm{Xe}$ are at maximum during the summer. In the summer the ocean warms due to subsurface solar heating. Krypton and Xe are less soluble in warmer water leading to a supersaturation and thus a flux of $\mathrm{Kr}$ and $\mathrm{Xe}$ out of the ocean. However, the flux is not strong enough to remove all the excess gas resulting in large positive saturation anomalies of up to $6 \%$ for $\mathrm{Kr}$ and $9 \%$ for $\mathrm{Xe}$ in the mixed layer. Below the mixed layer, the gases cannot be directly expelled to the atmosphere, resulting in large saturation anomalies that persist for months. The seasonal amplitude of $\mathrm{Kr}$ and Xe surface saturation anomalies are useful observational metrics for diffusive gas exchange, since as the gas exchange flux out of the mixed layer increases, $\Delta \mathrm{Kr}$ and $\Delta \mathrm{Xe}$ in the mixed layer decrease (Fig. 3-4).

Argon has a solubility in between that of $\mathrm{Ne}$ and $\mathrm{Kr}$ (Weiss, 1970) and thus has an intermediate response, responding both to air injection events (though more weakly than does $\mathrm{He}$ and $\mathrm{Ne}$ ) and to diffusive gas exchange. Argon is especially useful because it has a solubility and diffusivity similar to $\mathrm{O}_{2}$, allowing $\mathrm{Ar}$ to be an abiotic analogue of $\mathrm{O}_{2}$. The difference between $\mathrm{Ar}$ and $\mathrm{O}_{2}$ seen in Figure 3-2 is a direct result of biological production affecting only $\mathrm{O}_{2}$. In order to quantify biological production, we chose as an observational metric the difference between $\mathrm{Ar}$ and $\mathrm{O}_{2}$ saturation anomalies at $55 \mathrm{~m}$ depth. We found that a depth of $55 \mathrm{~m}$ for the saturation anomalies yielded the greatest sensitivity to biological production because $\Delta \operatorname{Ar}$ and $\Delta \mathrm{O}_{2}$ are at maxima at that depth during the summer.

By using observational metrics that incorporated all five noble gases and $\mathrm{O}_{2}$, we had six constraints. 
Figure 3-4: The Xe surface saturation anomaly for model runs with three different values of the model parameter $\gamma_{G}: \gamma_{G}=0.5(--), \gamma_{G}=1(-)$, and $\gamma_{G}=2(\ldots$.$) . The Xe surface saturation anomaly is a useful$ observational metric for gas exchange magnitude because it is sensitive to changes in $\gamma_{G}$. In the summer, Xe is supersaturated. As $\gamma_{G}$ increases, the flux of Xe out of the mixed layer increases, less Xe is left in the mixed layer, and thus $\Delta \mathrm{Xe}$ decreases.

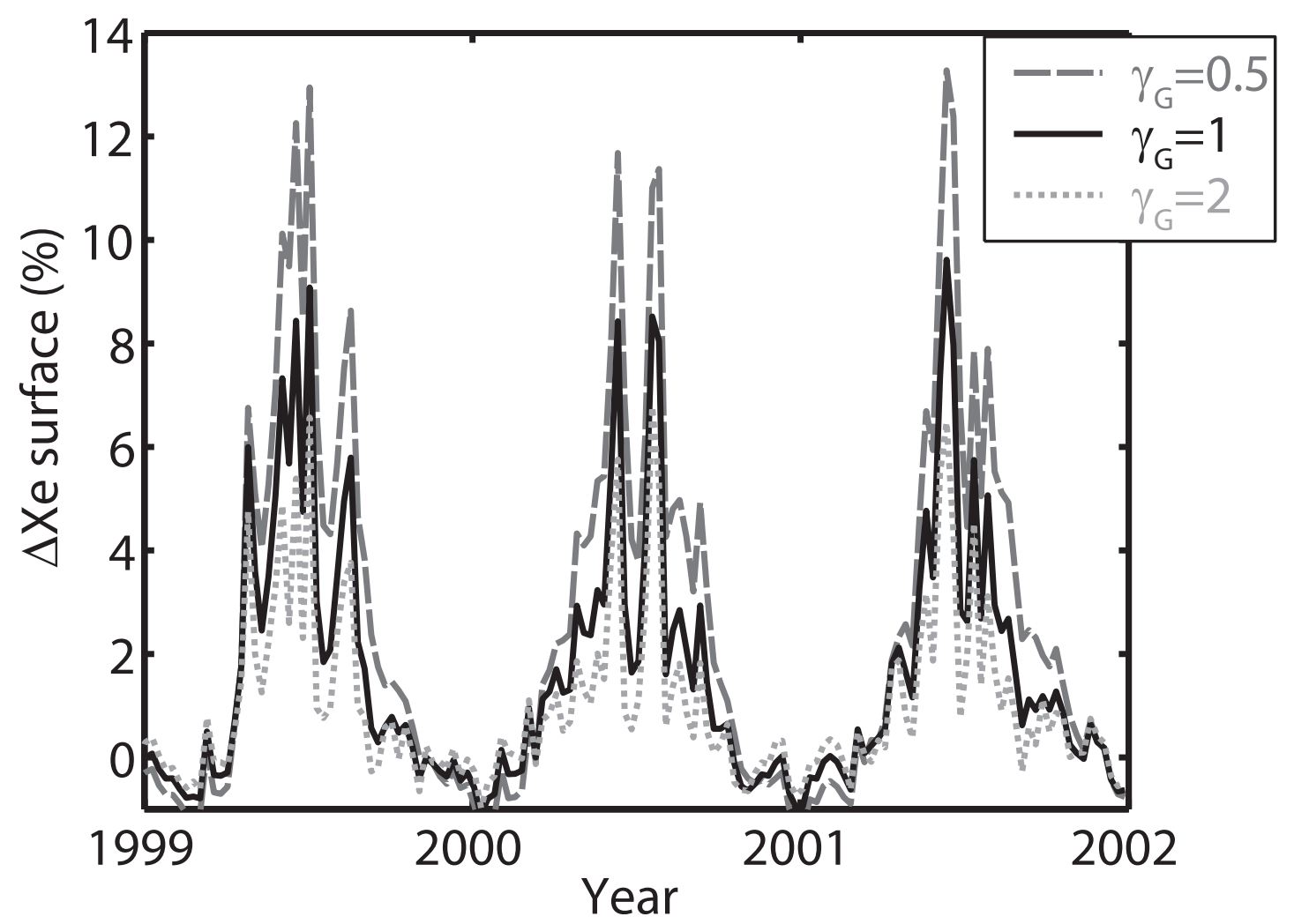


Additional constraints came from using the saturation anomalies of the gases in the surface or at any other depth, in the winter or the summer or annual average, or singly or in combination. Although not all of these constraints are strictly independent, multiple observational metrics allowed us to quantitatively distinguish among air-sea gas exchange processes.

\subsubsection{Quantifying the constraints}

The partial derivative method reveals which observational metrics are useful for constraining the air-sea gas exchange parameters. The constraints, slopes, and $r^{2}$ values of some of the observational metric/model parameter linear relationships are listed in Table 3.2. The suitability of an observational metric to the quantification of a given model parameter can readily be seen from the table or from a plot of the observational metric as a function of the model parameter (Fig. 3-5). A steeper slope of the linear regression corresponds to a stronger sensitivity of the observational metric to the parameter (see Eq. 3.11). For example, note that the He surface saturation anomaly vs. complete bubble trapping has a steep slope signifying a strong constraint, whereas the Xe surface saturation anomaly vs. complete bubble trapping has a very shallow slope signifying a weak constraint. Because the linearizations for complete bubble trapping are similar to those for partial bubble trapping, observational metrics that combined various noble gases were used to distinguish between complete and partial trapping. In particular, the difference between $\Delta \mathrm{Ne}$ and $\Delta \mathrm{Ar}$ provided a useful constraint for complete bubble trapping. For diffusive gas exchange magnitude, $\gamma_{G}$, the observational metrics were plotted as a function of $\gamma_{G}^{-1}$ since one would expect saturation anomalies of the gases to be inversely proportional to the strength of gas exchange. In addition, for $\gamma_{G}$, the slope is defined as the $\Delta$ (observational metric) $/ \Delta\left(\gamma_{G}^{-1}\right)$.

The advantage of the partial derivative method is that it allows determination of precisely which gases, or observational metrics, best constrain a given parameter. The disadvantage is the actual constraints determined from the partial derivative method are not necessarily accurate as they assume we have only one observational metric and only one model parameter at a time. Thus we need the full inverse method in order to determine simultaneously all of the constraints on air-sea gas exchange parameters as offered by a noble gas time-series (Table 3.3). Because we have multiple observational metrics, the constraints offered by the full inverse method are slightly tighter than those offered by the partial derivative method. For example, $\gamma_{G}$ 
Table 3.2: Uncertainty on model parameters as determined from individual observational metrics and the corresponding slopes as calculated from the partial derivative method. For each model parameter, the observational metrics that offer the tightest constraints on the parameter are listed as well as the slope, $\mathrm{r}^{2}$ value, and estimated measurement uncertainty in the observational metric. For a given model parameter, steeper slopes and smaller measurement uncertainties result in tighter constraints. Since $\gamma_{G}, \gamma_{A c}, \gamma_{A p}$ have the same reference values, it is possible to compare slopes between those parameters. For a definition of slope and constraint, see Eq. 3.11. The units of the slope are $\%$ for $\gamma_{G}, \gamma_{A c}, \gamma_{A p}$ and are $\% \mathrm{~mol}^{-1} \mathrm{~m}^{2}$ y for $\gamma_{P} . \Delta_{\text {surface }}$ refers to a surface saturation anomaly, $\Delta_{55}$ to saturation anomaly at $55 \mathrm{~m}$ depth, w to winter average, and s to summer average.

\begin{tabular}{|c|c|c|c|c|c|}
\hline $\begin{array}{c}\text { Model } \\
\text { Parameter }\end{array}$ & $\begin{array}{c}\text { Uncertainty of } \\
\text { model } \\
\text { parameter }\end{array}$ & $\begin{array}{l}\text { Observational } \\
\text { Metric }(\%)\end{array}$ & Slope & $\mathrm{r}^{2}$ & $\begin{array}{c}\text { Estimated } \\
\text { measurement } \\
\text { uncertainty in } \\
\text { observational } \\
\text { metric }(\%)\end{array}$ \\
\hline \multirow[t]{4}{*}{$\gamma_{G}$} & $6 \%$ & $\Delta \mathrm{Xe}_{\text {surface }} \mathrm{W}-\mathrm{s}$ & 3.5 & 0.996 & 0.2 \\
\hline & $6 \%$ & $\Delta \mathrm{Ar}_{\text {surface }} \mathrm{W}-\mathrm{s}$ & 1.5 & 0.998 & 0.1 \\
\hline & $8 \%$ & $\Delta \mathrm{Kr}_{\text {surface }} \mathrm{W}-\mathrm{s}$ & 2.4 & 0.997 & 0.2 \\
\hline & $17 \%$ & $\Delta \mathrm{He}_{\text {surface }} \mathrm{W}-\mathrm{s}$ & -0.61 & 0.995 & 0.1 \\
\hline \multirow[t]{5}{*}{$\gamma_{A c}$} & $20 \%$ & $\Delta \mathrm{Ne}_{\text {surface }} \mathrm{W}-\mathrm{S}$ & -0.49 & 1 & 0.1 \\
\hline & $22 \%$ & $\Delta \mathrm{He}_{\text {surface }} \mathrm{W}$ & -0.45 & 1 & 0.1 \\
\hline & $21 \%$ & $\Delta \mathrm{Ne}-\Delta \mathrm{Ar}_{\text {surface }} \mathrm{W}$ & 0.68 & 1 & 0.14 \\
\hline & $23 \%$ & $\Delta \mathrm{Ne}-\Delta \mathrm{Xe}_{\text {surface }} \mathrm{W}$ & 0.96 & 1 & 0.22 \\
\hline & $25 \%$ & $\Delta \mathrm{Ne}-\Delta \mathrm{Kr}_{\text {surface }} \mathrm{W}$ & 0.85 & 1 & 0.22 \\
\hline \multirow[t]{3}{*}{$\gamma_{A p}$} & $26 \%$ & $\Delta \mathrm{He}_{\text {surface }} / \Delta \mathrm{Ne}_{\text {surface }}$ & 0.38 & 0.995 & 0.1 \\
\hline & $36 \%$ & $\Delta \mathrm{He}_{\text {surface }} \mathrm{W}-\mathrm{s}$ & -0.28 & 0.9997 & 0.1 \\
\hline & $39 \%$ & $\Delta \mathrm{Ne}_{\text {surface }} \mathrm{W}-\mathrm{s}$ & -0.25 & 0.9998 & 0.1 \\
\hline$\gamma_{P}$ & $0.2 \mathrm{~mol} \mathrm{O}_{2} \mathrm{~m}^{-2} \mathrm{y}^{-1}$ & $\Delta \mathrm{Ar}_{55}-\Delta \mathrm{O}_{2,55} \mathrm{~s}$ & 1.1 & 1 & 0.22 \\
\hline
\end{tabular}


Figure 3-5: The Linearizations: the observational metrics are plotted as a function of model parameter and then a linear relationship is calculated. The slope is a reflection of how sensitive the observational metric is to the parameter - a steep slope implies a sensitive metric. The observational metrics are plotted as a function of the inverse of the gas exchange parameter $\gamma_{G}$ because the saturation anomalies of the noble gases are inversely proportional to gas exchange.
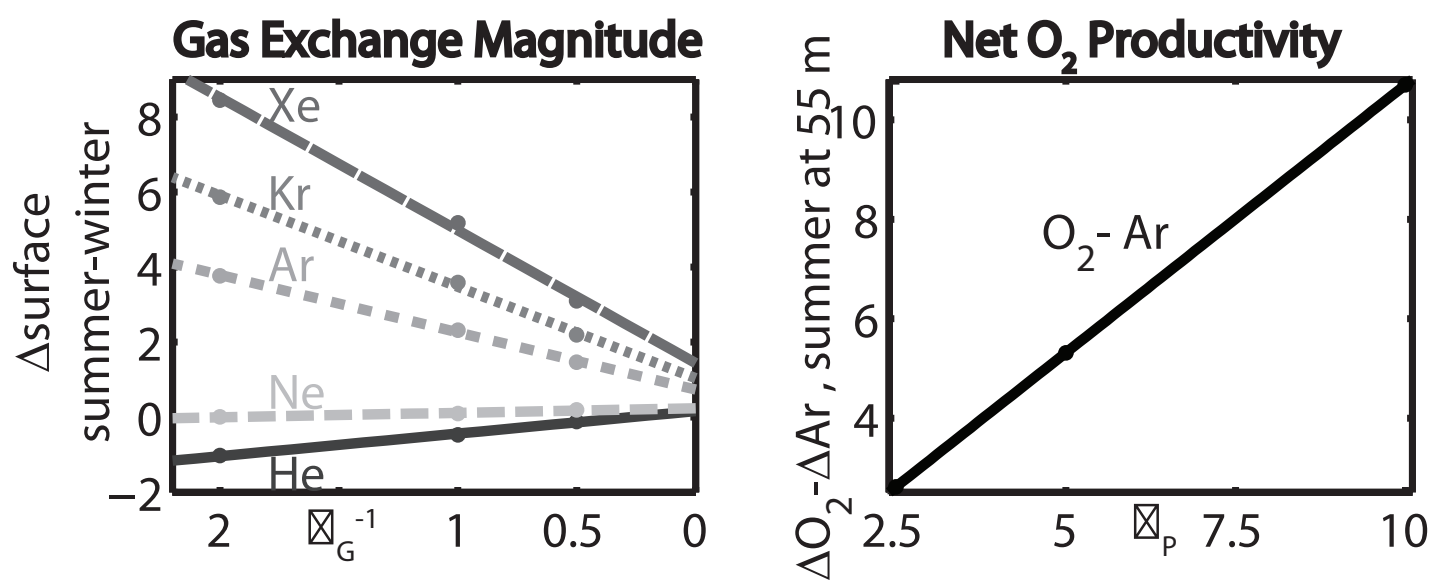

\section{Complete Bubble Trapping}
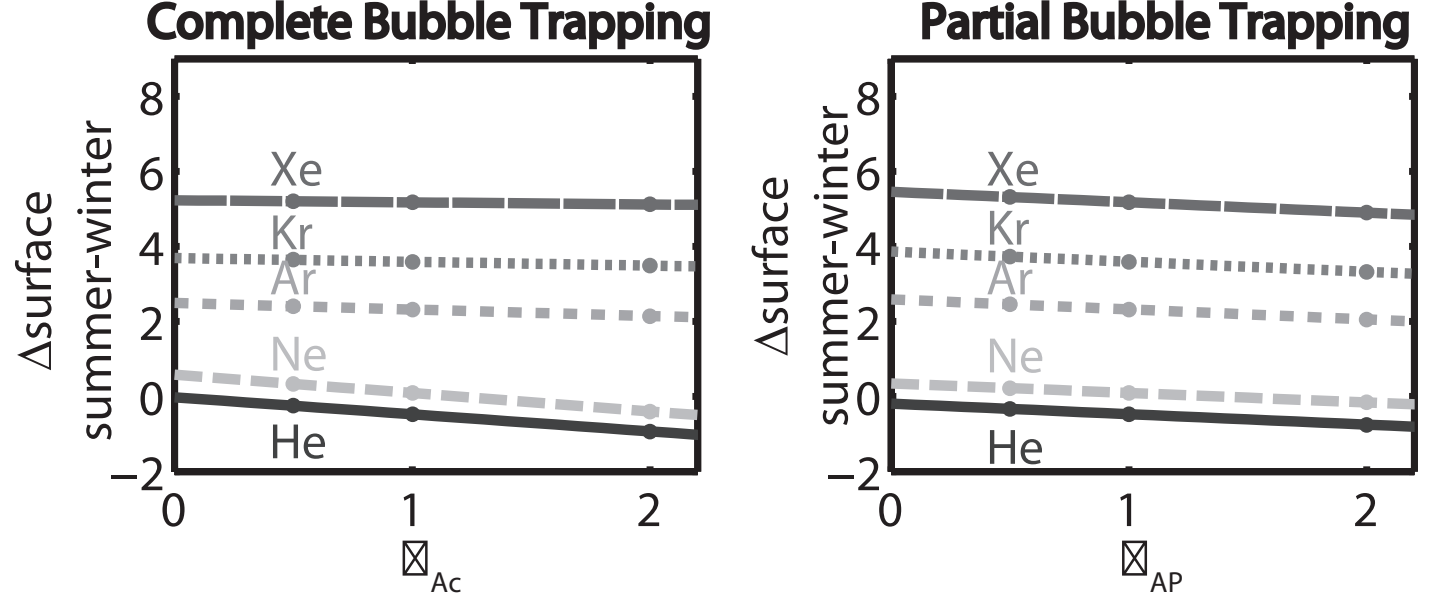
Table 3.3: Constraints on the air-sea gas exchange model parameters calculated using the full inverse method. All uncertainties are $1 \sigma$ values. First listed is the uncertainty of the model parameter with a fixed choice of physical parameters, second the additional uncertainty due to the choice of physical parameters (i.e. uncertainty due to 1D model physics), and third the total uncertainty on the parameter, calculated by adding the above uncertainties in quadrature. Because there is a large negative covariation between partial and complete trapping, the total air injection flux, $\mathrm{F}_{A I}$, has an uncertainty of approximately $14 \%$.

\begin{tabular}{cccc}
\hline $\begin{array}{c}\text { Model } \\
\text { Parameter }\end{array}$ & $\begin{array}{c}\text { Uncertainty } \\
\text { from full } \\
\text { inverse method }\end{array}$ & $\begin{array}{c}\text { Uncertainty } \\
\text { from physical } \\
\text { parameters }\end{array}$ & $\begin{array}{c}\text { Total } \\
\text { Uncertainty }\end{array}$ \\
\hline$\gamma_{G}$ & $4 \%$ & $10 \%$ & $11 \%$ \\
$\gamma_{A c}$ & $12 \%$ & $17 \%$ & $20 \%$ \\
$\gamma_{A p}$ & $21 \%$ & $15 \%$ & $26 \%$ \\
$\gamma_{P}\left(\mathrm{~mol} \mathrm{O}_{2} \mathrm{~m}^{-2} \mathrm{y}^{-1}\right)$ & 0.2 & 1.5 & 1.5 \\
\hline
\end{tabular}

can be constrained to $6 \%$ in the partial derivative method and to $4 \%$ in the full inverse method. When we include the additional uncertainty due to choice of physical parameters, the total uncertainty for $\gamma_{G}$ is $11 \%$. Estimates of net biological production could be constrained to $\pm 0.2 \mathrm{~mol} \mathrm{O}_{2} \mathrm{~m}^{-2} \mathrm{y}^{-1}$ if $K_{z}$ were known perfectly. However, uncertainties in $K_{z}$ increase the uncertainty in net biological production to $1.5 \mathrm{~mol} \mathrm{O}_{2}$ $\mathrm{m}^{-2} \mathrm{y}^{-1}$. Biological production is sensitive to the choice of $\mathrm{Kz}$ because there is a very sharp gradient in $\mathrm{O}_{2}$, but not in Ar, below the euphotic zone. In this respect, Ar is not a perfect abiogenic analog of oxygen.

The magnitude of the complete bubble trapping flux, $F_{C}$, can be constrained to $12 \%$ (20\% if one includes the uncertainty due to possible variations in physical parameters), and of magnitude of the partial bubble trapping flux, $F_{P}$, to $23 \%$ (26\% including the uncertainty due to physical parameters), both significant improvements over other currently available methods. However, even with multiple gases, we have some difficulty in resolving the partitioning between partial trapping and complete trapping. The uncertainty in the total air injection flux, $F_{A I}$, is smaller than the uncertainty of either $F_{C}$ or $F_{P}$ because there is a large negative covariation between $\gamma_{A c}$ and $\gamma_{A p}$. The uncertainty on total air injection depends upon the gas, being smaller for the gases that are more influenced by air injection. For $\mathrm{He}, \mathrm{Ne}$, and Ar the uncertainty on $F_{A I}$ is $6 \%$. For $\mathrm{Kr}$ and $\mathrm{Xe}$, the uncertainty on $F_{A I}$ is $8 \%$ and $10 \%$ respectively. The choice of physical parameters adds another $12 \%$ uncertainty. The total uncertainty in air injection, averaged for the five gases, including 
uncertainty due to physical parameters, is $14 \%$. If we use a more pessimistic (and less realistic estimate) of the possible measurement uncertainties of the gases of $0.3 \%$ for all measurements, we can constrain $\gamma_{G}$ to $\pm 13 \%, \gamma_{A c}$ to $\pm 29 \%, \gamma_{A p}$ to $\pm 51 \%$ and $\gamma_{P}$ to $\pm 1.5 \mathrm{~mol} \mathrm{~m}^{-2} \mathrm{y}^{-1}$ (these uncertainties include the uncertainty due to physical parameters).

Ideally, one would use this method to determine $n$, the power dependency of gas exchange magnitude on wind speed. The exact nature of the wind-speed dependency of the gas transfer velocity is not well-resolved. It may be a quadratic relationship (Wanninkhof, 1992), a cubic one (Wanninkhof and McGillis, 1999), or one following some fractional exponent. Unfortunately however, Bermuda is not an ideal spot to study wind speed dependency. The quadratic and cubic parameterizations are scaled to yield the same flux at the global average wind speed $\left(7.4 \mathrm{~m} \mathrm{~s}^{-1}\right)$ in order to match the global radiocarbon balance (Wanninkhof, 1992; Wanninkhof and McGillis, 1999). The average wind speed in Bermuda (7.1 $\mathrm{m} \mathrm{s}^{-1}$ for the period 1999-2002) is similar to the global average wind speed, making it difficult to distinguish between a quadratic and cubic parameterization. Additionally, the wind speed distribution in Bermuda has a standard deviation of only 3.4 $\mathrm{m} \mathrm{s}^{-1}$, and there are very few resolved large wind-speed events that would show a large difference between quadratic and cubic parameterizations.

We performed calculations with $n=3, n=2$, and $n$ as a tunable model parameter. The estimated uncertainties of the air-sea gas exchange parameters are similar whether we use $n=3$ or $n=2$. The value for the coefficient for the gas exchange flux (i.e. $7.9 \times 10^{-8}$ in Eq. 3.2) and the value of $\gamma_{G}$ will change if a quadratic or cubic form is used but the relative uncertainty to which we can constrain $\gamma_{G}$ remains similar. If we use $n$ as a tunable model parameter, we can determine the power dependency to \pm 0.4 . However, due to the interplay of all the parameters in the flux equations, the constraints on the other parameters are weakened to $\pm 15 \%$ for $\gamma_{G}$, $\pm 27 \%$ for $\gamma_{A c}$, and $\pm 28 \%$ for $\gamma_{A p}$. For example, since the diffusive gas exchange flux $F_{G E}$ depends on both $n$ and $\gamma_{G}$, if $n$ can vary, then $\gamma_{G}$ must have a larger uncertainty as well. Although this method can in principle determine $n$, extrapolating a determination of $n$ made at the low wind speeds in Bermuda to higher wind speeds is unwarranted. Hence, in Bermuda, where there are low winds and only a small spread in wind-speed, it is best to fix $n$ and determine the best value of $\gamma_{G}$ for $n=2$ and $\gamma_{G}$ for $n=3$. Researchers who wish to use parameterizations of gas exchange determined by this method could choose an $\mathrm{n}$ that is appropriate for their study site and then use the corresponding coefficient and $\gamma_{G}$ as determined by 
this method.

We explored the sensitivity of these conclusions to assumptions of the power dependence of solubility and diffusivity ( $a$ and $b$ in Eq. 3.5), to choice of solubility functions and to selection of reference case values. If different combinations of $a$ and $b$ are used ( $a=1, b=2 / 3 ; a=1, b=1 / 2 ;$ or $a=0.7, b=0.35)$, the values of $\gamma_{A c}$ and $\gamma_{A p}$ change. However, the fractional constraints offered by the noble gases on the parameters do not differ significantly. Thus we should be able to use noble gases to constrain air-sea gas exchange parameterizations regardless of the functional form of the air injection equation. The choice of solubility function, those of Weiss $(1970,1971)$ or of Hamme and Emerson (2004b), does not significantly affect how well the noble gases can be used to constrain air-sea gas exchange parameterizations in the sensitivity analysis. However, the choice of solubility function does affect the actual values for the parameters and thus it is essential to have accurate solubility functions for all the noble gases.

The fractional constraints on $\gamma_{G}$ and $\gamma_{P}$ remain the same regardless of reference case parameter values. However, the constraints on $\gamma_{A c}$ and $\gamma_{A p}$ change with reference case. $\gamma_{A c}$ and $\gamma_{A p}$ covary and thus some reference cases have larger $\gamma_{A c}$ and smaller $\gamma_{A p}$, resulting in a larger proportion of air injection due to complete trapping. In that case, the constraint on complete trapping becomes tighter but the constraint on partial trapping becomes looser. Overall, the constraint on total air injection remains $14 \%$ regardless of reference case.

\subsection{Example application: Time-series of helium, neon, and argon}

As a limited demonstration, we performed an example determination of air-sea gas exchange parameters using a monthly time-series of $\mathrm{He}, \mathrm{Ne}, \mathrm{Ar}$, and $\mathrm{O}_{2}$ measurements made at Station $\mathrm{S}$ from March, 1985 to February, 1988 (Spitzer, 1989; Spitzer and Jenkins, 1989). The results of this example analysis are limited because the data are only for three of the noble gases and yet above we show that it is necessary to have the heavier noble gases $(\mathrm{Kr}$ and $\mathrm{Xe})$ in order to separate diffusive gas exchange and air injection. In addition, methods for collecting and measuring noble gas samples have improved greatly (Lott and Jenkins, 1998), so measurements made today would be of higher quality. Nonetheless, the exercise allows us a preliminary glance at seasonal air-sea gas exchange parameters in an oligotrophic subtropical gyre. For the Spitzer (1989) data, samples for He and Ne were collected from Niskin bottles in copper tubes containing 
$45 \mathrm{~g}$ of seawater and were measured mass spectrometrically using peak height manometry. Samples for Ar were collected in copper tubes containing $13 \mathrm{~g}$ of seawater and were measured using isotope dilution mass spectrometry. For a more complete descriptions of methods, see Spitzer and Jenkins (1989). The noble gas data is presented in Figure 3-6. The $1 \sigma$ analytical uncertainty of Ar measurements is $0.4 \%$ but the reproducibility of the samples from samples drawn from the same Niskin bottle is about $1 \%$. The $1 \sigma$ analytical uncertainty for $\mathrm{He}$ and $\mathrm{Ne}$ measurements is $0.2 \%$ and the reproducibility is similarly $1 \%$. (For comparison, in Section 3.4 for the sensitivity study, we assumed modern errors of $0.1 \%$. The errors are smaller in modern studies because of improved sampling procedures and because of averaging made possible by oversampling in space and time). We attribute the larger scatter of this data to artifacts associated with the early sampling method. In addition, some of the samples with large supersaturations may have been contaminated with bubbles trapped during sample collection.

We used the full inverse method with twelve observational metrics to quantify the four model parameters: $\gamma_{G}, \gamma_{A c}, \gamma_{A p}$, and $\gamma_{P}$. The observational metrics and associated $1 \sigma$ errors are listed in Table 3.4. Since the metrics are the mean of data over a given period, the errors are based on the combined standard error of the estimate of the mean (major component) and the analytical uncertainty (minor component). The error also includes the uncertainty in the solubility functions for all observational metrics that are not differences between two saturation anomalies (either the difference between winter and summer values or between one gas and another since in both such cases any systematic uncertainties in solubility should cancel). For uncertainty in solubility functions, we use $1.5 \%, 0.3 \%$ and $0.13 \%$ for $\mathrm{He}, \mathrm{Ne}$ and Ar respectively (Hamme and Emerson, 2004b). The He uncertainty is determined by the size of the correction we applied to the Weiss solubility functions.

The coefficient matrix, created from the slopes of the linearizations and weighted by the $1 \sigma$ errors in the observational metrics, is listed in Table 3.5. In a given column, a large absolute value of a coefficient implies the observational metric has a large part in determining the parameter. From the coefficient matrix, one can see $\mathrm{Ar}$ is more sensitive to diffusive gas exchange and less sensitive to complete bubble trapping than are $\mathrm{He}$ and Ne. Nonetheless, all three gases are sensitive to all the air-sea gas exchange parameters, and thus, as expected, having only $\mathrm{He}, \mathrm{Ne}$, and Ar makes it difficult to quantitatively distinguish between air injection and gas exchange. In order to increase our ability to separate the two processes, we used additional 
Figure 3-6: Monthly surface saturation anomalies (o) of $\mathrm{He}, \mathrm{Ne}$, and $\mathrm{Ar}$ at Station S, collected and measured by Spitzer (1989). The model results (-) are constructed using the model parameters determined from the full inverse calculation.
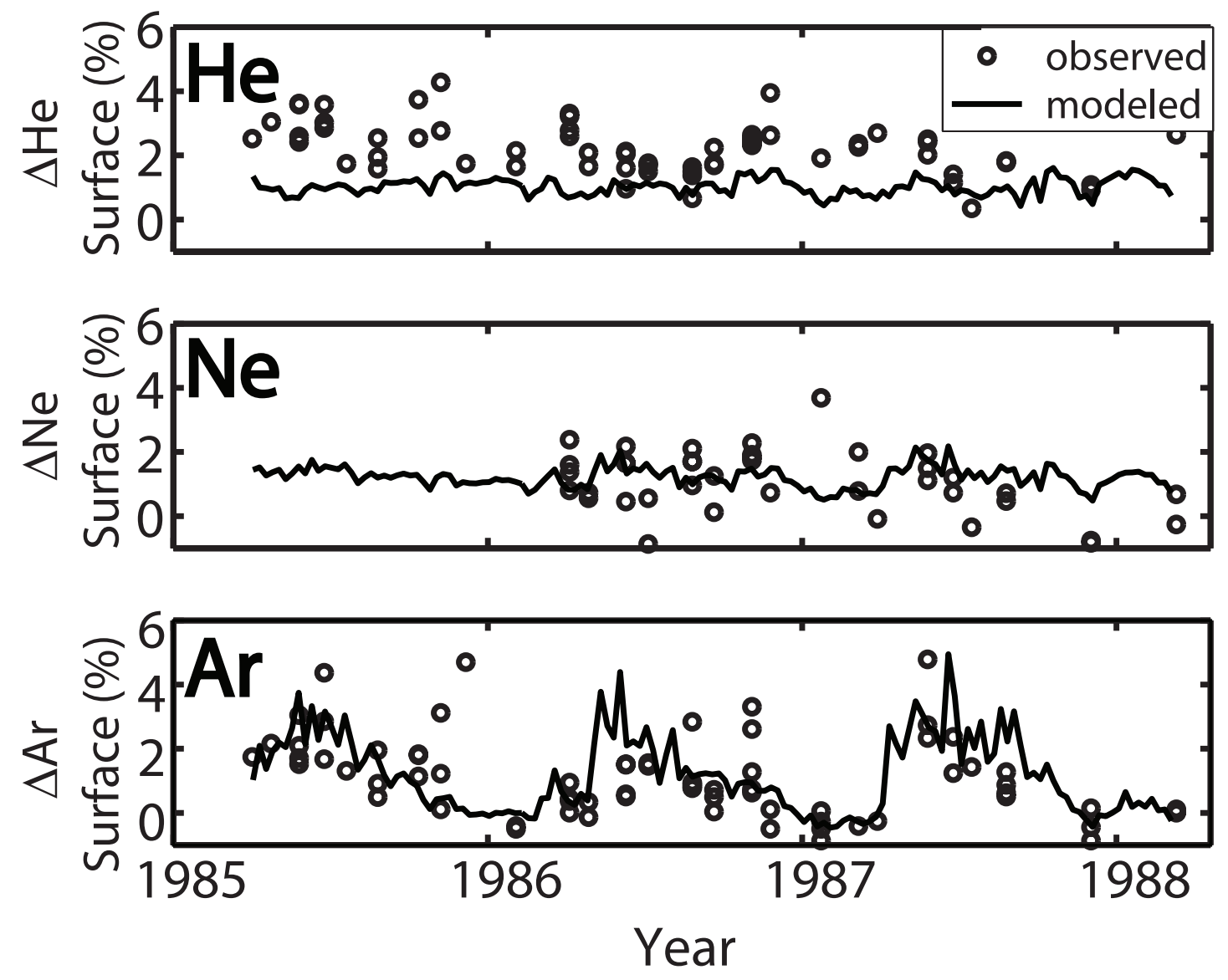
Table 3.4: Twelve observational metrics were calculated from the limited time series of $\mathrm{He}, \mathrm{Ne}, \mathrm{Ar}$ and $\mathrm{O}_{2}$ collected from 1985-1988 in the Sargasso Sea (Spitzer, 1989). The $1 \sigma$ errors listed are the combined error due to the standard error of the estimate of the mean, the analytical uncertainty, and the solubility uncertainty if appropriate (see text for details). The model run was constructed using the air-sea gas exchange model parameters determined from the inverse calculation. $\Delta_{\text {surface }}$ refers to a surface saturation anomaly, $\Delta_{50}$ to saturation anomaly at $50 \mathrm{~m}$ depth, $\mathrm{w}$ to winter average, $\mathrm{s}$ to summer average, and a to annual average. The units of all saturation anomalies are \%.

\begin{tabular}{ccc}
\hline Observational Metric & Data & Model \\
\hline$\Delta \mathrm{He}_{\text {surface }} \mathrm{S}-\mathrm{W}$ & $-0.19 \pm 0.4$ & -0.02 \\
$\Delta \mathrm{Ne}_{\text {surface }} \mathrm{S}-\mathrm{W}$ & $-0.56 \pm 0.8$ & 0.54 \\
$\Delta \mathrm{Ar}_{\text {surface }} \mathrm{S}-\mathrm{w}$ & $2.3 \pm 0.3$ & 2.5 \\
$\Delta \mathrm{O}_{50}-\Delta \mathrm{Ar}_{50} \mathrm{~S}$ & $11 \pm 1$ & 11 \\
$\Delta \mathrm{Ar}_{50} \mathrm{~S}-\mathrm{W}$ & $3.3 \pm 0.7$ & 4.9 \\
$\Delta \mathrm{He}_{\text {surface }} \mathrm{W}$ & $2.3 \pm 0.4$ & 1.0 \\
$\Delta \mathrm{Ne}_{\text {surface }} \mathrm{W}$ & $1.4 \pm 0.8$ & 0.93 \\
$\Delta \mathrm{Ar}_{\text {surface }} \mathrm{W}$ & $-0.27 \pm 0.2$ & -0.05 \\
$\Delta \mathrm{He}_{-} \mathrm{Ne}_{\text {surface }} \mathrm{a}$ & $1.0 \pm 0.3$ & -0.2 \\
$\Delta \mathrm{He}_{\text {surface }} \mathrm{a}$ & $2.2 \pm 0.9$ & 1.0 \\
$\Delta \mathrm{Ne}_{\text {surface }} \mathrm{a}$ & $1.0 \pm 1.1$ & 1.2 \\
$\Delta \mathrm{Ar}_{\text {surface }} \mathrm{a}$ & $1.1 \pm 0.3$ & 1.1 \\
\hline
\end{tabular}


Table 3.5: The slope matrix created from the linearizations of the analysis of the $\mathrm{He}, \mathrm{Ne}, \mathrm{Ar}$, and $\mathrm{O}_{2}$ timeseries data, weighted by the $1 \sigma$ errors in the observational metrics. The observational metrics are listed in the first column. The parameters investigated are listed in the first row. A larger slope implies the observational metric responds strongly to changes in the parameter. $\Delta_{\text {surface }}$ refers to a surface saturation anomaly, $\Delta_{50}$ to saturation anomaly at $50 \mathrm{~m}$ depth, w to winter average, s to summer average, and a to annual average.

\begin{tabular}{ccccc}
\hline Obs. Metric $\backslash$ Parameter & $\gamma_{G}$ & $\gamma_{A c}$ & $\gamma_{A p}$ & $\gamma_{P}$ \\
\hline$\Delta \mathrm{He}_{\text {surface }} \mathrm{S}-\mathrm{W}$ & -1.9 & -1.3 & -1.0 & 0 \\
$\Delta \mathrm{Ne}_{\text {surface }} \mathrm{S}-\mathrm{w}$ & -0.5 & -0.6 & -0.4 & 0 \\
$\Delta \mathrm{Ar}_{\text {surface }} \mathrm{s}-\mathrm{W}$ & 2.9 & -0.6 & -1.0 & 0 \\
$\Delta 2_{50}-\Delta \mathrm{Ar}_{50} \mathrm{~s}$ & 0.1 & 0 & 0 & 1.1 \\
$\Delta \mathrm{Ar}_{50} \mathrm{~s}-\mathrm{W}$ & 0.6 & -0.1 & -0.2 & 0 \\
$\Delta \mathrm{He}_{\text {surface }} \mathrm{W}$ & 3.8 & 2.7 & 1.3 & 0 \\
$\Delta \mathrm{Ne}_{\text {surface }} \mathrm{W}$ & 1.9 & 1.5 & 0.6 & 0 \\
$\Delta \mathrm{Ar}_{\text {surface }} \mathrm{W}$ & 2.5 & -0.6 & 0.1 & 0 \\
$\Delta \mathrm{He}_{-} \mathrm{Ne}_{\text {surface }} \mathrm{a}$ & -0.7 & 2.5 & 2.3 & 0 \\
$\Delta \mathrm{He}_{\text {surface }} \mathrm{a}$ & 1.3 & 0.9 & 0.3 & 0 \\
$\Delta \mathrm{Ne}_{\text {surface }} \mathrm{a}$ & 1.2 & 0.9 & 0.2 & 0 \\
$\Delta \mathrm{Ar}_{\text {surface }} \mathrm{a}$ & 0.7 & 0.3 & 0.2 & 0 \\
\hline
\end{tabular}

observational metrics. For example, the seasonal amplitude of the saturation anomaly of Ar at $50 \mathrm{~m}$ depth is less influenced by air injection then the other metrics since air injection is less important for more soluble gases such as Ar. Other observational metrics used include the difference between saturation anomalies of $\mathrm{O}_{2}$ and $\mathrm{Ar}$ at $50 \mathrm{~m}$ depth in order to constrain biological production and the difference between $\mathrm{He}$ and Ne surface saturation anomalies to constrain the fraction of bubbles completely vs. partially trapped. We also included the winter surface saturation anomalies of $\mathrm{He}, \mathrm{Ne}$, and $\mathrm{Ar}$ since these winter values are more sensitive to air injection and diffusive gas exchange than the seasonal amplitudes. Thus the winter values are valuable even though they have larger associated $1 \sigma$ errors (in order to take into account the error in solubility functions). 
Table 3.6: Model parameter values as determined by the full inverse method (SVD) using the $\mathrm{He}, \mathrm{Ne}, \mathrm{Ar}$ and $\mathrm{O}_{2}$ time-series data.

\begin{tabular}{ccc}
\hline Model & Parameter & Description Reference Value \\
\hline$\gamma_{G}$ & Magnitude of diffusive gas exchange & $0.7 \pm 0.7$ \\
$\gamma_{A c}$ & Magnitude of complete trapping component of air injection & $0.5 \pm 1$ \\
$\gamma_{A p}$ & Magnitude of partial trapping component of air injection & $0.4 \pm 1$ \\
$\gamma_{P}$ & Magnitude of net biological production & $9 \pm 3 \mathrm{~mol} \mathrm{O}_{2} \mathrm{~m}^{-2} \mathrm{y}^{-1}$ \\
\hline
\end{tabular}

With twelve observational metrics and four parameters, the system is over-determined and can be solved using SVD to yield "best" values for the four tunable model parameters (Table 3.6). Uncertainties on the parameter values were determined from propagating the $1 \sigma$ error from the observational metrics through the SVD calculation and by including the error due to choice of physical parameters. Values of the air-sea gas exchange model parameters are robust to choice of physical parameters but differ by $50 \%$ according to whether the Hamme and Emerson (2004b) solubility functions or the Weiss (1970, 1971); Weiss and Kyser (1978) solubility functions are used. Though the total air injection fluxes are robust, the partitioning of these fluxes between complete and partial trapping is sensitive to choice of reference case. The magnitude of the biological production is sensitive to the choice of $K_{z}$. When we performed our calculations over a range of $K_{z}\left(2 \times 10^{-5}\right.$ to $\left.8 \times 10^{-5} \mathrm{~m}^{2} \mathrm{~s}^{-1}\right)$, the estimated biological production varied by $30 \%$. We included this source of error in the calculation of the $1 \sigma$ error of $\gamma_{P}$. Additionally, there is some ambiguity in the absolute magnitude of all parameters due to potential biases and errors associated with the NCEP reanalysis winds that were used to force the model.

Overall, the model parameters determined are reasonable. The gas exchange magnitude is $70 \% \pm 70 \%$ of that predicted by Wanninkhof and McGillis (1999). The net biological production is $9 \pm 3 \mathrm{~mol} \mathrm{O}_{2} \mathrm{~m}^{-2}$ $\mathrm{y}^{-1}$, which is equivalent to $6 \pm 2 \mathrm{~mol} \mathrm{C} \mathrm{m}^{-2} \mathrm{y}^{-1}$ or $0.9 \pm 0.3 \mathrm{~mol} \mathrm{~N} \mathrm{~m}^{-2} \mathrm{y}^{-1}$ using the revised Redfield ratios of Anderson and Sarmiento (1994). Although this estimate is based on an idealized oxygen profile, it is similar to a recent estimate of new production determined using the ${ }^{3} \mathrm{He}$ flux gauge approach of 0.84 $\pm 0.26 \mathrm{~mol} \mathrm{~N} \mathrm{~m}^{-2} \mathrm{y}^{-1}$ (Jenkins and Doney, 2003). It is slightly higher than several previous biogeochem- 
ical estimates of production in the Sargasso Sea (Jenkins and Goldman, 1985; Jenkins, 1988a; Spitzer and Jenkins, 1989; Gruber et al., 1998).

We investigated how well the model could match the data by using the best parameters as determined from the SVD (Table 3.6) to run the model. The resulting model run results are plotted with the data in Figure 3-6. Observational metrics calculated from the model run are listed in Table 3.4. The model predicts He saturation anomalies consistently lower than the observed He, perhaps because of systematic errors in sampling collection, sampling analysis or in the solubility of He. The model run allows us to quantify and compare the fluxes due to diffusive gas exchange, complete bubble trapping, and partial bubble trapping (Fig. 3-7). Although $\mathrm{Kr}$ and $\mathrm{Xe}$ were not measured, the model can use the air-sea gas exchange parameters determined from the $\mathrm{He}, \mathrm{Ne}$, and Ar data to calculate diffusive gas exchange and air injection fluxes for $\mathrm{Kr}$ and $\mathrm{Xe}$ as well. For the insoluble gases $\mathrm{He}$ and $\mathrm{Ne}$ (not shown), the diffusive gas exchange flux is similar in magnitude and opposite in direction to the air injection flux. Large air injection events are mirrored by large diffusive gas exchange fluxes. Additionally, the air injection flux is primarily due to complete trapping. For the more soluble gases $\mathrm{Kr}$ (not shown) and $\mathrm{Xe}$, the opposite is true. The diffusive gas exchange flux is larger than the air injection flux and there are many diffusive gas exchange events that are not associated with air injection events. Because these gases are soluble with a strong temperature dependence to solubility, air injection is less important and diffusive gas exchange is primarily driven by thermal forcing. The air injection flux of $\mathrm{Kr}$ and $\mathrm{Xe}$ is primarily due to partial trapping. The behavior of Ar is intermediate between He and Xe.

We explored the sensitivity of the calculations to the power dependency of solubility and diffusivity used in the partial bubble trapping equation ( $a$ and $b$ in Eq. 3.5). We repeated all calculations for three different cases of $a$ and $b\left(\alpha D^{2 / 3}, \alpha D^{1 / 2}, \alpha^{0.7} D^{0.35}\right)$. If $\alpha D^{1 / 2}$ or $\alpha^{0.7} D^{0.35}$ is used, then negative values for $\gamma_{A p}$ result from the SVD calculation. This situation is clearly unphysical. One can, however, achieve positive values for all parameters if one does not use the two observational metrics of winter average $\Delta \mathrm{Ar}$, and $\Delta \mathrm{He}$ $\Delta \mathrm{Ne}$. This implies that in the $\alpha D^{1 / 2}$ or $\alpha^{0.7} D^{0.35}$ cases, it is possible to achieve the general signatures of the gases but is impossible to match the winter average of $\Delta \mathrm{Ar}$ or to achieve the proper differences between the saturation anomalies of $\mathrm{He}$ and $\mathrm{Ne}$. If one uses the results from the SVD where those two observational metrics were excluded, the total fluxes for air injection and gas exchange are a factor of two larger than 
Figure 3-7: Model results for the diffusive gas exchange flux $F_{G E}$ (gray), total air injection flux $F_{A I}$ (black), and partial air injection flux $F_{P}$ (dashed) for $\mathrm{He}, \mathrm{Ar}$, and Xe. The complete air injection flux $F_{C}$ is equal to the difference between the $F_{A I}$ and $F_{P}$ curves. The fluxes are calculated using the parameter values determined from the full inverse method. Note the difference in vertical scales used for each gas. For He, the gas exchange flux is approximately equal in magnitude and opposite in sign to the air injection flux whereas for Xe, the gas exchange flux is typically much larger than the air injection flux.
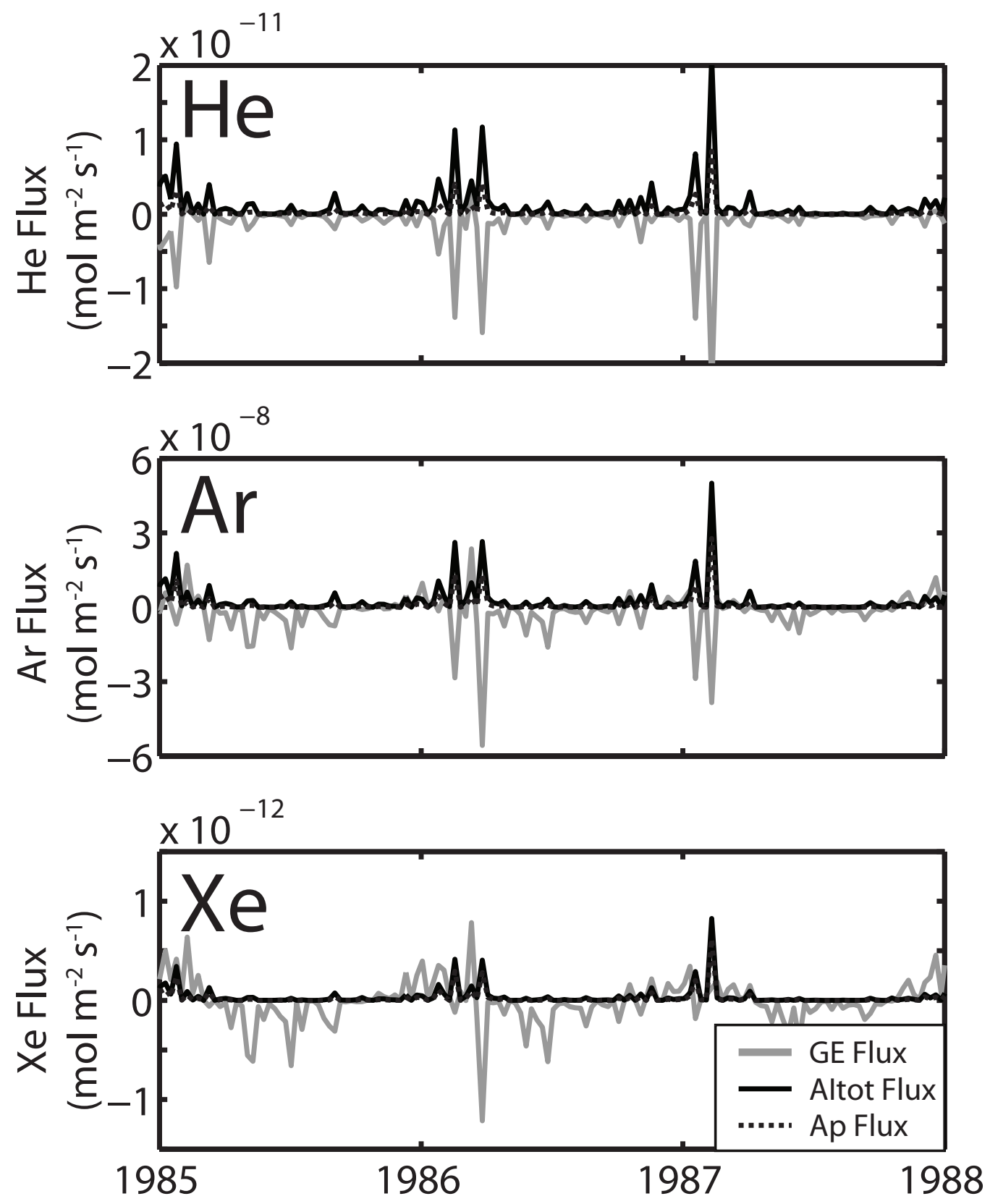
the results for $\alpha D^{2 / 3}$ case calculated with all observational metrics. The results for $\alpha D^{1 / 2}$ or $\alpha^{0.7} D^{0.35}$ are similar to the results for a calculation performed with $\alpha D^{2 / 3}$ but without the same two observational metrics. Thus both choice of observational metrics and power dependency of $\alpha$ and $D$ are important. Differences in power dependency are not merely notational - they have important implications for the total fluxes of gases. Since isotopes have similar solubilities but different diffusivities, isotopic ratios of the noble gases (e.g. ${ }^{20} \mathrm{Ne} /{ }^{22} \mathrm{Ne},{ }^{36} \mathrm{Ar} /{ }^{40} \mathrm{Ar}$ ) could potentially be used to better constrain the power dependency of the diffusivity of partial bubble trapping.

The exercise performed here differs from the work of Spitzer and Jenkins (1989) in several ways. We used NCEP reanalysis winds and heat fluxes to force the model, a cubic parameterization of equilibrium gas exchange, and a different formulation of air injection processes. Spitzer and Jenkins used climatological winds and heat fluxes, a three-step linear parameterization of equilibrium gas exchange (Liss and Merlivat, 1986), and a formulation of air injection where partial bubble trapping depended only on the diffusivity of the gas. In addition, Spitzer and Jenkins did not use Ne data and used only one year of $\mathrm{He}, \mathrm{Ar}$, and $\mathrm{O}_{2}$ data. Our results differ from Spitzer and Jenkins in that we find that the majority of bubbles are completely trapped, whereas they found that partial trapping was dominant. The biological production we estimate is 45\% larger than the Spitzer and Jenkins estimate. It is difficult to directly compare estimates of gas exchange since we use different parameterizations. The uncertainties on the model parameters are quite large - much larger than the expected constraints on model parameters from the theoretical noble gas sensitivity study that we report in Section 3.4. These large uncertainties result from the limited nature of the data. First, we only have data for three noble gases and yet above we show we need all five noble gases to separate the air injection and diffusive gas exchange processes. Second, the data itself has large uncertainties associated with sample collection and measurements. Current methods for sample collection and measurement have improved greatly in the last two decades. In the expected constraints reported in Section 3.4, we assumed measurement uncertainties of $0.1 \%$ for $\mathrm{He}, \mathrm{Ne}$ and $\mathrm{Ar}$, and $0.2 \%$ for $\mathrm{Kr}$ and $\mathrm{Xe}$. Hence the large uncertainties in this exercise do not contradict the results of Section 3.4. Rather, they show that though we have a powerful method, we need to have the appropriate data in order to use the method. Additionally, some uncertainty may result from the real ocean being more complex than our ideal model assumes. 


\subsection{Conclusions}

Noble gases are useful tools for quantifying air-sea gas exchange parameters since the difference in physical properties of the gases allows separation of processes. Model calculations show that a time-series of all five noble gases and $\mathrm{O}_{2}$ can constrain air-sea gas exchange parameters more tightly than can other currently available methods. An additional advantage of this noble gas method is that it allows the study of gas exchange from empirical data on a larger scale than is possible with purposeful tracer release or laboratory experiments.

The potential constraints on the air-sea gas exchange parameters were calculated using only the uncertainty due to expected measurement capability and to choice of physical parameters. Additional sources of potential uncertainty that were not included in the analysis but would serve to increase the calculated uncertainties (loosen the constraints) of parameters include variability in sample collection due to small-scale processes such as Langmuir cells and mesoscale processes such as eddies. We can try to average over the small-scale processes by taking multiple samples in the mixed layer and over the mesoscale processes by using a multi-year time-series with monthly resolution. Furthermore, satellite imagery can be used to assess the influence of eddies and lateral processes.

Systematic uncertainties include a bias against sampling in high-wind events and any errors in the solubility functions of the noble gases. The former is mitigated by noble gas signatures integrating over a few weeks so samples only need be collected within a few weeks of the storm event. In addition samples can be collected with a moored noble gas sampler that can collect samples in any conditions (Hood, 1998). However, wind speed data can be problematic at high wind speeds, thus making interpretation of the noble gas data difficult. Although systematic biases in solubility can be partially mitigated by using seasonal amplitudes of the noble gases saturation anomalies, this study illustrates the need for accurate determination of the solubilities of all the noble gases.

As no data yet exists for all five noble gases, we presented an example calculation using a time-series of only three of the noble gases in order to illustrate the method and to calculate some initial values for air-sea gas exchange parameters in the Sargasso Sea. Ongoing work includes collection of a time-series of all five noble gases in order to exploit the full potential of this method. Current improvements in sample collection and extraction (Lott and Jenkins, 1998) and in mass spectrometry, including advances in the technique of 
using cryogenics to separate the noble gases (Lott, 2001), make such a time-series feasible. In April 2003, we started measuring a three-year time series of all five noble gases. That data combined with the method presented here (as well as with a full nonlinear optimization) will allow an accurate determination of air-sea gas exchange parameters based on empirical measurements. These estimates are unique in that they cover the time-scale (several weeks to months rather than days or years) and spatial scale (regional average rather than local patch or global scope) that is important to understanding the biogeochemical cycling of gases. 


\subsection{Acknowledgments}

This work was supported by the National Science Foundation Chemical Oceanography program (OCE0221247) and by the Department of Defense (graduate fellowship to RHRS). We would like to thank Don Rice for his support and encouragement, Roberta Hamme for the use of MATLAB code for the solubility functions of the noble gases, and Burkard Baschek and Dierdre Toole for useful discussions. We also thank Michael Bender and Steven Emerson for their helpful reviews. 
Table 3.7: Appendix: Definition of symbols and variables

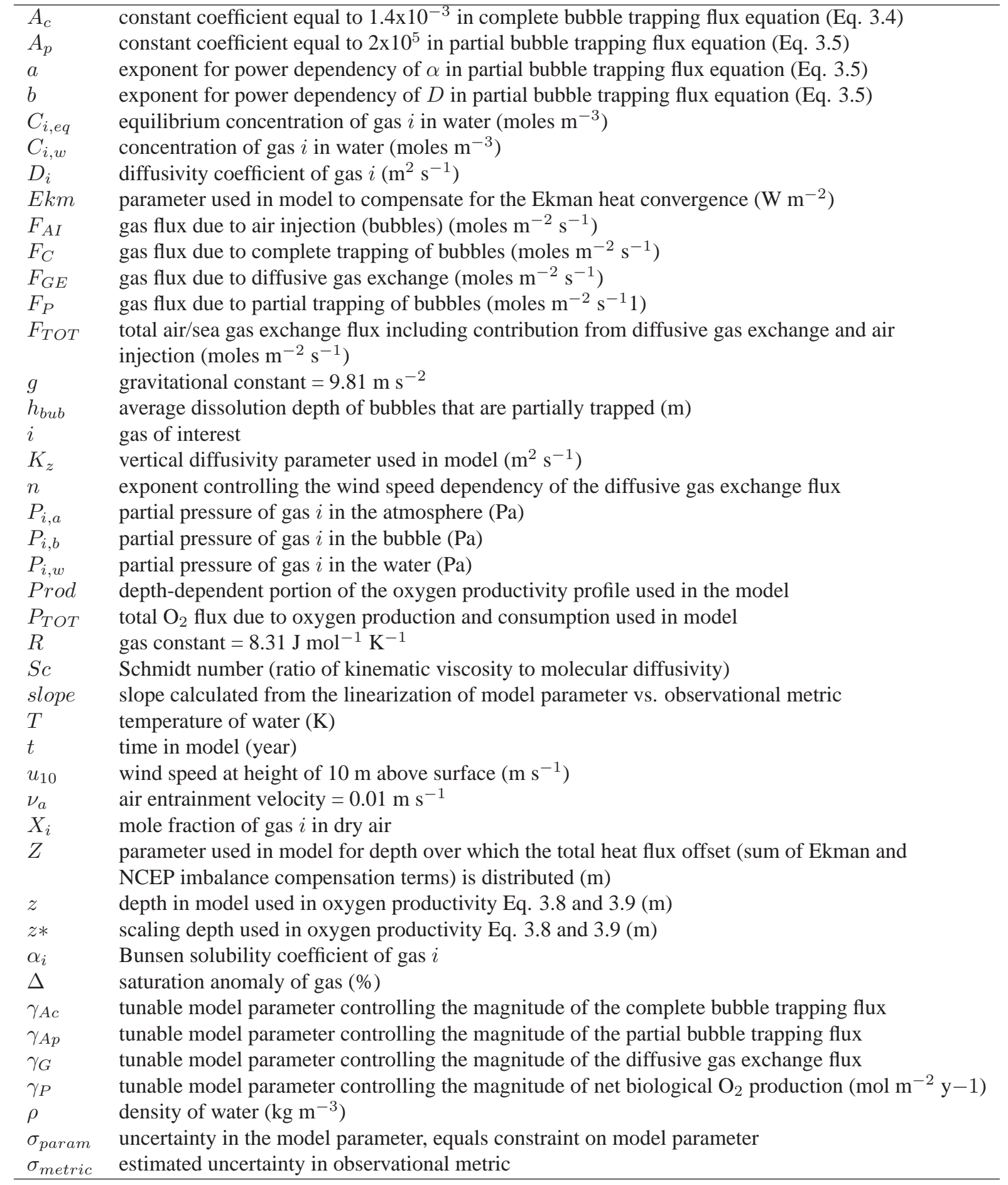


Chapter 4

Air-Sea Gas Exchange Parameters as

Determined by a Time-Series of Five Noble

Gases 


\begin{abstract}
A fourteen month time-series at approximately monthly resolution of five noble gases (helium, neon, argon, krypton, and xenon) collected in the upper $400 \mathrm{~m}$ in the Sargasso Sea is used in conjunction with an upper ocean mixed layer model to separate and quantify diffusive gas exchange to $\pm 6 \%$, and complete and partial bubble trapping to $\pm 15 \%$. We present parameters to be used with standard formulations of these three airsea gas exchange processes in oligotrophic waters over the range of wind speeds encountered during this time-series $\left(0<\mathrm{u}_{10}<13 \mathrm{~m} \mathrm{~s}^{-1}\right)$. The magnitude of diffusive gas exchange is determined to be $79 \%$ of the value proposed by Wanninkhof (1992). The magnitude of air injection in the winter is significant for all gases. The winter air injection flux of Ar is comparable in magnitude to the diffusive gas exchange flux. The fraction of air injection associated with completely trapped bubbles (as opposed to partially trapped ones) ranges from $91 \%$ for $\mathrm{He}$ to $70 \%$ for $\mathrm{Xe}$, and is estimated to be approximately $86 \%$ for oxygen. The largest uncertainties in our determination of the parameters come from uncertainties in the solubilities of the heavier noble gases and from potential biases in the upper ocean physical model.
\end{abstract}




\subsection{Introduction}

Air-sea gas exchange is a crucial component of the biogeochemical cycle of many climatically relevant gases such as $\mathrm{CO}_{2}, \mathrm{O}_{2}$, DMS, $\mathrm{N}_{2} \mathrm{O}$, and $\mathrm{CH}_{4}$. Accurate representation of air-sea gas exchange processes in climate models is clearly important. Air-sea gas exchange parameterizations are used in order to estimate $\mathrm{CO}_{2}$ fluxes from maps of surface ocean $\mathrm{pCO}_{2}$, (Takahashi et al., 1997). Additionally, quantification of air-sea gas exchange fluxes is necessary for many types of biogeochemical research, such as in studies of euphotic zone oxygen measurements for determining net production (Craig and Hayward, 1987; Emerson, 1987; Spitzer and Jenkins, 1989), ${ }^{3} \mathrm{He}$ measurements for determining new production (Jenkins, 1988b; Jenkins and Doney, 2003), and $\mathrm{O}_{2} / \mathrm{N}_{2}$ and APO calculations (Keeling et al., 1993; McKinley et al., 2003; Bender et al., 2005).

Many parameterizations of air-sea gas exchange exist (Liss and Merlivat, 1986; Wanninkhof, 1992; Wanninkhof and McGillis, 1999; Nightingale et al., 2000) but such parameterizations have large uncertainties. Recently, two parameterizations (Ho et al., 2006; Sweeney et al., 2007) predict air-sea fluxes that are $25 \%$ to 33\% lower than Wanninkhof (1992) proposed. Existing parameterizations are based on either large spatial and temporal scales (such as the global radiocarbon budget) or on local spatial and short time scales (such as with tracer release experiments and edddy-correlation measurements). Additionally, most existing parameterizations do not explicitly include air injection (bubble) processes.

Bubble-mediated gas transfer may be significant, especially for less soluble gases and at higher wind speeds. It is difficult to estimate the total air injection flux from theoretical treatment of bubbles (Woolf and Thorpe, 1991; Woolf, 1993; Keeling, 1993) because of uncertainties in bubble size spectra, bubble dynamics, and depth distribution. A few parameterizations of bubble processes have been formulated (Asher and Wanninkhof, 1998; Zhang et al., 2006; Hamme and Emerson, 2006), allowing estimation of bubble flux given fraction of white-cap coverage. These formulations have large uncertainties and in many cases do not distinguish between bubbles that have been completely dissolved, and therefore inject atmospheric abundances, versus bubbles that only partially dissolve before rising to the surface, thus fractionating the gases (Fuchs et al., 1987; Jenkins, 1988a).

Noble gases are ideal tracers for investigating air-sea gas exchange because they are chemically and biologically inert. Additionally, they have a wide range of solubilities (order of magnitude) and molecular diffusivities (factor of five) and thus respond differently to physical processes. The lighter noble gases have 
been used previously to estimate air-sea gas exchange parameters. Spitzer and Jenkins (1989) used a timeseries of $\mathrm{He}, \mathrm{Ne}$, and $\mathrm{Ar}$ in order to estimate the rate of diffusive gas exchange and air injection. More recently, Hamme and Emerson (2006) used a time series of $\mathrm{Ne}$, Ar, and $\mathrm{N}_{2}$ to investigate the partitioning of completely trapped vs. partially dissolved bubbles. The study presented here expands on previous work by including measurements of $\mathrm{Kr}$ and $\mathrm{Xe}$. Since $\mathrm{Kr}$ and $\mathrm{Xe}$ are the most soluble noble gases and have the strongest temperature-dependence of solubility, they greatly improve the constraints on both diffusive gas exchange and on air injection fluxes (Stanley et al., 2006).

In this work, we use a 14 month time-series at approximately monthly resolution of five noble gases measured in the upper $400 \mathrm{~m}$ of the water column in the Sargasso Sea in order to construct a parameterization of air-sea gas exchange based on direct empirical data that has a characteristic time-scale of weeks to seasons - the time-scale relevant to many biogeochemical cycles in the ocean - and that explicitly includes bubble processes. Simple calculations of mixed layer depths and gas transfer velocity show that the time-scale of gas exchange flux of the noble gases is on the order of weeks. Additionally, our estimates are based on gas saturations driven by seasonal warming. By using all five noble gases, the relatively insoluble helium $\mathrm{He}$ and $\mathrm{Ne}$ as well as the more soluble and temperature dependent $\mathrm{Ar}, \mathrm{Kr}$, and $\mathrm{Xe}$, we are able to separate and quantify diffusive gas exchange to $\pm 6 \%$ and air injection processes $\pm 15 \%$. The parameters presented here are valid for use in environments with similar wind conditions to the Sargasso Sea $\left(0<\mathrm{u}_{10}<13 \mathrm{~m} \mathrm{~s}^{-1}\right)$ and that are similarly oligtrophic and thus have minimal surfactants. We combine our noble gas time-series with a one-dimensional mixed layer model (described in section 4.2) and a non-linear constrained optimization scheme in order to use the time-series to quantify air-sea gas exchange parameters. In section 4.3, we present the noble gas data and the air-sea gas exchange parameters as determined by inverse modeling. In section 4.4, we examine the controls on the parameters exerted by the various noble gases and the sensitivity of the parameters to the physical parameters in our model, to the choice of cost function, and to uncertainties in the solubilities of the noble gases. 


\subsection{Methods}

\subsubsection{Data Collection}

Samples for noble gas analysis were collected aboard the $R / V$ Weatherbird II, the $R / V$ Cape Hatteras, or the $R / V$ Oceanus at the Bermuda Atlantic Time-series Study (BATS) site every month between July 2004 and August 2005. On all cruises except for the February 2005 cruise, the samples were collected on a "Core" BATS cruise. The exact dates and location of the samples are listed in Table 4.1. The samples were collected from Niskin bottles by gravity feeding through tygon tubing into valved stainless steel sample cylinders (90 cc volume). The cylinders were gently rapped during sampling in order to dislodge bubbles. We extracted the gases from the samples into aluminosilicate glass bulbs (approximate volume of bulb is $25 \mathrm{cc}$ ) in the on-shore laboratory at the Bermuda Biological Station. Details of the sampling and extraction procedures are described in Lott and Jenkins (1998).

Samples were extracted as soon as possible - usually within 24 hours of sample collection - because $\mathrm{He}$ and to a lesser extent $\mathrm{Ne}$ permeate through the viton o-rings in the cylinder plug valves. Experiments conducted with degassed water suggest that samples are compromised at a rate of 0.0046 and 0.0009 of their disequlibrium per day for $\mathrm{He}$ and $\mathrm{Ne}$ respectively. Thus for a sample with a 5\% disequilbrium in $\mathrm{He}$ and $\mathrm{Ne}$, a 24 hour delay in extraction would lead to a concentration shift of $0.023 \%$ and $0.005 \%$ respectively. The aluminosilicate bulbs were brought to Woods Hole Oceanographic Institution and stored in ambient conditions for up to two years before analysis. Because the gases have a practically zero permeation rate through aluminosilicate glass, this storage time should have no effect on the measurements.

The aluminosilicate bulbs are attached to a dual mass spectrometric system and analyzed for $\mathrm{He}, \mathrm{Ne}$, $\mathrm{Ar}, \mathrm{Kr}$, and $\mathrm{Xe}$ as well as for helium isotopes. For details of analytical procedures, please see Chapter 2 of this thesis. In brief, the noble gases in the water sample are chemically purified by being sequentially drawn through a two-stage water vapor cryotrap to remove water vapor, through a Pd catalyst to remove methane, and through Ti-Zr-Fe getters to remove $\mathrm{O}_{2}, \mathrm{~N}_{2}, \mathrm{CO}_{2}$, and other active gases. The noble gases are then drawn onto two cryogenic traps - a stainless steel cryogenic trap for $\mathrm{Ne}, \mathrm{Ar}, \mathrm{Kr}$, and $\mathrm{Xe}$, and an activated charcoal cryogenic trap for He. The cryogenic traps are selectively warmed and the gases are sequentially released into the statically operated quadrupole mass spectrometer (QMS) for measurement by 
peak height manometry. The QMS is a Hiden quadrupole mass spectrometer (P/N PCI 1000 1.2HAL/3F 1301-9 PIC type 570309) run in static mode, equipped with a pulse counting secondary electron multiplier (SEM) run at an emission of 20 to $40 \mu \mathrm{amps}$, an electron impact ion source and triple quadrupole ion optics. Standardization of the system is accomplished using precisely known aliquots of atmospheric noble gases (determined by pressure, temperature, volume and relative humidity), and thus is dependent on knowledge of the abundance of these gases in air. However, since studies involving dissolved gases generally presume these values, uncertainties in these abundances cancel out in most of the following calculations.

Our data are presented here as saturation anomalies, $\Delta$, which are the percent deviation from solubility equilibrium, and here defined as

$$
\Delta=\left(\frac{C_{i, w}}{C_{i, e q}}-1\right) \times 100
$$

where $C_{i, w}$ is the concentration of gas $i$ in the water and $C_{i, e q}$ is the concentration of gas $i$ at equilibrium (i.e. the solubility value). Positive (negative) saturation anomalies reflect that the gas is supersaturated (undersaturated), and the magnitude of the saturation anomalies corresponds to the magnitude of the departure from equilibrium. The solubilities of many of the noble gases were determined by Weiss in the 1970s (Weiss, 1970, 1971; Weiss and Kyser, 1978). More recently, the solubilities of Ne and Ar were redetermined by Hamme and Emerson (2004b), with these newer results being larger than those of Weiss by $1 \%$ for $\mathrm{Ne}$ and $0.4 \%$ for Ar. We perform the calculations with the $\mathrm{Ne}$ and Ar solubilities of Hamme and Emerson. We assumed that the difference in the Ne solubilities between Weiss and Hamme and Emerson was due to a systematic difference in technique. We thus scaled the Weiss solubility values for He (which was not determined by Hamme and Emerson) with the same correction factor that was needed to make the Weiss $\mathrm{Ne}$ solubility equal to the Hamme and Emerson Ne solubility. This correction factor, which depends on temperature, ranges from $+0.5 \%$ at $18^{\circ} \mathrm{C}$ to $+1.5 \%$ at $29^{\circ} \mathrm{C}$. For $\mathrm{Kr}$, we use the solubility value determined by Weiss and Kyser (1978) and for Xe, the solubility value determined by Wood and Caputi (1966). We propagated the uncertainty in solubility determination throughout all the calculations.

Bubbles can easily be trapped during sampling in the void spaces in the plug valves in the stainless steel cylinders and on the inner walls of the cylinders. We try to reduce this by rapping the cylinder walls and by exercising (opening and closing) the plug valves on order 20 times during sample collection. Nonetheless, 
it may be impossible to completely eliminate bubbles. In order to assess the amount of gas trapped in our samples due to bubbles entrained during sample collection, we measured the noble gases in distilled water that had been equilibrating with laboratory air for 24 hours. (For details of this test, please see Chapter 2 of this thesis.) We then calculated the saturation state of these samples using Krause and Benson (1989) solubility values for distilled water and found the average saturation anomalies to be $101.1 \pm 0.8 \%$ for $\mathrm{He}, 99.5 \pm 0.7 \%$ for Ne, $99.3 \pm 0.7 \%$ for Ar, $98.5 \pm 1 \%$ for $\mathrm{Kr}$, and $98.6 \pm 1.2 \%$ for Xe. We assumed the deviations from $100 \%$ saturation were results of a combination of air entrainment from bubbles (which affects the lighter, less soluble gases more strongly than the heavier, more soluble ones), and thermal effects resulting from incomplete thermal equilibration or temperature gradients within the reservoir (which affects the heavier, more soluble gases the most). We scaled the contribution from bubbles so that each gas is affected according to atmospheric concentration and solubility considerations. We then solved a series of 5 linear equations, one for each gas, for these two effects and calculated the saturation anomaly due to bubbles in our samples (Table 4.2), assuming there is no inherent error in the Benson and Kraus solubility values. We subtract this saturation anomaly from all our subsequent results in order to compensate for small amounts of bubbles inadvertently collected in our samples. We performed this experiment in the early stages of our analysis and our method has improved since then, most significantly for $\mathrm{Ne}, \mathrm{Kr}$, and $\mathrm{Xe}$. We will thus repeat this experiment at a later point, now that we have a better analytical technique. Additionally, we will use a temperature controlled bath and a longer equilibration time in order to mitigate temperature effects.

Several other corrections were applied to our samples. Approximately 135 samples were analyzed before we started directly measuring the fraction of $\mathrm{Ne}$ that was inadvertently and unavoidably drawn onto the charcoal trap and including this contribution as part of our Ne measurements. Although early tests showed quantitative trapping of $\mathrm{Ne}$ on the stainless steel cryotrap, subsequent and gradual degradation of the minimum cryotrap temperature led to less than perfect $\mathrm{Ne}$ retention, and consequent transfer of $\mathrm{Ne}$ to the charcoal trap. We corrected these samples by assuming $0.68 \%$ of Ne would have been released from the charcoal trap for standards and $1.02 \%$ of Ne would have been released from the charcoal trap for samples. These correction factors were obtained by averaging the fraction of Ne released from the charcoal trap in samples and standards run with the same procedure but where Ne was measured from the charcoal trap. This correction, however, is not well-known since the amount of Ne released from the charcoal cryotrap may 
Table 4.1: Details of sample collection.

\begin{tabular}{ccccc}
\hline Date & Lat & Long & BATS cruise \# & vessel \\
\hline $07-14-2004$ & 31.691 & 64.166 & 190 & $R / V$ Weatherbird II \\
$08-18-2004$ & 31.682 & 64.162 & 191 & $R / V$ Weatherbird II \\
$09-15-2004$ & 31.667 & 64.164 & 192 & $R / V$ Weatherbird II \\
$10-15-2004$ & 31.667 & 64.167 & 193 & $R / V$ Weatherbird II \\
$11-13-2004$ & 31.668 & 64.166 & 194 & $R / V$ Weatherbird II \\
$12-09-2004$ & 31.668 & 64.168 & 195 & $R / V$ Weatherbird II \\
$01-28-2005$ & 31.699 & 64.187 & 196 & $R / V$ Cape Hatteras \\
$02-23-2005$ & 31.667 & 64.167 & - & $R / V$ Oceanus \\
$03-22-2005$ & 31.669 & 64.166 & 198 & $R / V$ Weatherbird II \\
$04-20-2005$ & 31.677 & 64.17 & 199 & $R / V$ Weatherbird II \\
$05-18-2005$ & 31.668 & 64.169 & 200 & $R / V$ Weatherbird II \\
$06-15-2005$ & 31.654 & 64.185 & 201 & $R / V$ Weatherbird II \\
$07-20-2005$ & 31.668 & 64.165 & 202 & $R / V$ Weatherbird II \\
$08-11-2005$ & 31.666 & 64.167 & 203 & $R / V$ Weatherbird II \\
\hline
\end{tabular}

differ depending on the recent history of the trap, a factor our correction does not capture. Additionally, 40 samples may have additional uncertainty in $\mathrm{Kr}$ measurements because initially, we may not have adequately corrected for the matrix effects that Ar and Xe have on Kr.

Even after these corrections, certain samples have extremely large or small saturation anomalies, reflecting perhaps extreme bubble entrainment during sampling, leaky Niskins, or under-extractions. We plotted a histogram of all our data and then chose saturation anomaly limits beyond which to exclude data points. If the $\Delta \mathrm{He}$ was less than $-5 \%$ or greater than $6 \%$, we excluded the samples. If $\Delta \mathrm{Xe}$ was less than $-5 \%$ or greater than $20 \%$, we also excluded the samples. These criterion resulted in a rejection of 14 data-points, approximately $5 \%$ of our data. 
Table 4.2: Contribution of bubbles entrained in samples during sample collection, as derived from distilled water tests, and expressed as a saturation anomaly. The saturation anomalies are consistent with the relative effect of air injection on the noble gases given the differences in solubility between the gases (i.e. if bubble entrainment creases a $1.17 \%$ supersaturation in $\mathrm{He}$, then it also will create a $0.084 \%$ supersaturation in Xe). The standard deviation of the supersaturation due to bubbles in the eleven test distilled water samples is listed as well.

\begin{tabular}{ccc}
\hline Gas & Sat Anom from Bubbles & Std. Dev. \\
\hline $\mathrm{He}$ & $1.17 \%$ & $0.8 \%$ \\
$\mathrm{Ne}$ & $0.97 \%$ & $0.7 \%$ \\
$\mathrm{Ar}$ & $0.29 \%$ & $0.2 \%$ \\
$\mathrm{Kr}$ & $0.15 \%$ & $0.1 \%$ \\
$\mathrm{Xe}$ & $0.084 \%$ & $0.06 \%$ \\
\hline
\end{tabular}

\subsubsection{Description of the one-dimensional vertical upper ocean model}

We use a one-dimensional, vertical, modified Price-Weller-Pinkel (PWP) model (Price et al., 1986). The model previously has been extended to include $\mathrm{He}, \mathrm{Ne}, \mathrm{Ar}$, and $\mathrm{O}_{2}$ (Spitzer and Jenkins, 1989) and to include $\mathrm{Kr}$ and $\mathrm{Xe}$ (Stanley et al., 2006). For details on the model, please see Stanley et al. (2006) or chapter 3 of this thesis. Here only a brief description of the model is included.

The model is forced with wind stress from the QuikSCAT satellite and with heat fluxes from the National Centers for Environmental Prediction (NCEP) reanalysis (Kistler et al., 2001), interpolated for the BATS site. The attenuation of radiative heat is governed by a Jerlov Type 1A profile. QuikSCAT winds were chosen over NCEP winds because the QuikSCAT winds agree more closely with a wind record from the nearby Bermuda Testbed Mooring (BTM) (Dickey et al., 1998). The correlation coefficient between the NCEP and BTM record was 0.64 whereas the correlation coefficient between the QuikSCAT and BTM record was 0.82. The BTM wind record was not used directly because of gaps when the mooring was not deployed. Additionally, a significant advantage of using QuikSCAT winds is to make our parameterization more applicable for other applications, since satellite data are more frequently available than mooring data.

The surface temperature in the model is restored every 6 hours to a smoothed, interpolated record of 
the BATS sea surface temperature, with a restoring constant of $75 \mathrm{~W} \mathrm{~m}^{-2} \mathrm{deg} \mathrm{C}^{-1}$. The annually averaged NCEP net heat flux for this site is negative, reflecting net cooling. Lateral advection probably balances the local heat budget over decadal time-scales. To compensate for this effect, we add to the model an upper ocean positive heat flux of order $50 \mathrm{~W} \mathrm{~m}^{-2}$, calculated by balancing the sum of the NCEP heat fluxes to zero. In addition, in the model there is an effective heat convergence associated with Ekman pumping. We computed the Ekman pumping from the four day low pass filtered local wind stress curl derived from the NCEP reanalysis data. The Ekman pumping induced vertical velocity is tapered to zero at the bottom of the model domain, in approximate concordance with the Sverdrup relation. The vertical heat flux convergence associated with this flux results in a net heat gain in the model. To prevent the model from warming, a heat flux of order $20 \mathrm{~W} \mathrm{~m}^{-2}$ is removed from the water column. This Ekman heat convergence compensation term $(E k m)$ depends on the evolution of model temperature and thus is difficult to calculate explicitly. It is therefore treated as a model parameter that is adjusted for long term heat balance. Similarly, the depth to which the total heat offset (sum of lateral and Ekman compensation terms) is distributed $(Z)$ is treated as a tunable model parameter. Additionally, the vertical diffusivity below the mixed layer in the model, $K_{z}$ is also treated as a tunable model parameter.

Ensemble runs $(\mathrm{n} \sim 100)$ and a cost function based on the root mean square difference between model and data temperature and salinity (with data from the BATS bottle casts) were used to determine optimal values for these physical parameters $\left(E k m, Z, K_{z}\right)$. A wide range of physical parameters was examined: $2 \times 10^{-5}<K_{z}<2 \times 10^{-4} \mathrm{~m}^{2} \mathrm{~s}^{-1}, 1<E k m<30 \mathrm{~W} \mathrm{~m}^{-2}$, and $10<Z<125 \mathrm{~m}$. The parameters work in concert, and thus several sets of parameters yield almost equivalent cost function values (Table 4.3). Subsequent work uses primarily the base case with a low cost function, i.e, $K_{z}=5 \times 10^{-5} \mathrm{~m}^{2} \mathrm{~s}^{-1}, E k m=18$ $\mathrm{W} \mathrm{m}^{-2}$, and $Z=50 \mathrm{~m}$, but we also explore the sensitivity of our results to other sets of physical parameters.

For these studies, the model was spun up from January, 2002 with initial conditions of temperature and salinity profiles from BATS data for that date and initial gas saturation anomalies of $2.45 \%$ for $\mathrm{He}, 2.75 \%$ for $\mathrm{Ne}, 1.7 \%$ for $\mathrm{Ar}, 1.48 \%$ for $\mathrm{Kr}$, and $1.55 \%$ for Xe at all depths. To choose these initial gas values, the model was run many times with various initial conditions. Each time, the model converged to similar values for the deep water and those values were chosen to be our initial conditions. Furthermore, when these values were used as initial conditions, the deep values in the model did not drift. These values are within the error 
Table 4.3: The three best sets of physical parameters. "rmsd" refers to the root mean square difference of temperature in the upper $200 \mathrm{~m}$ of the water column between the model and BATS data from 2003 to 2006.

\begin{tabular}{cccc}
\hline $\begin{array}{c}K_{z} \\
\left(\mathrm{~m}^{2} \mathrm{~s}^{-1}\right)\end{array}$ & $\begin{array}{c}E k m \\
\left(\mathrm{~W} \mathrm{~m}^{-2}\right)\end{array}$ & $\begin{array}{c}Z \\
(\mathrm{~m})\end{array}$ \\
\hline $5 \times 10^{-5}$ & 18 & 50 & 0.664 \\
$6 \times 10^{-5}$ & 20 & 50 & 0.665 \\
$4 \times 10^{-5}$ & 16 & 50 & 0.670 \\
\hline
\end{tabular}

limits of our winter saturation anomalies, further justifying their choice. In any case, the model results did not change significantly if different starting saturation anomalies or a longer spinup were used.

\subsubsection{Gas exchange parameterization used in the model}

Gas exchange processes are modeled according to standard literature parameterizations with adjustment permitted through the use of tunable model parameters. The treatment of gas exchange processes is identical to Stanley et al. (2006) (chapter 3 of this thesis), with the exceptions that (1) rather then using $\gamma_{A c}$ and $\gamma_{A p}$, we directly investigate the values of $A_{c}$ and $A_{p}$ and (2) a quadratic parameterization of diffusive gas exchange on wind speed is used. The equations are described in full in Stanley et al. (2006) and are only briefly described here.

The net total flux of a gas, $F_{T O T}$, is the sum of the flux due to diffusive gas exchange, $F_{G E}$, and the flux due to air injection, $F_{A I}$ :

$$
F_{T O T}=F_{G E}+F_{A I}
$$

The diffusive gas exchange flux, $F_{G E}\left(\mathrm{~mol} \mathrm{~m}^{-2} \mathrm{~s}^{-1}\right)$ is given by

$$
F_{G E}=\gamma_{G} \cdot k_{G}\left(\frac{S c_{i}}{660}\right)^{-0.5} u_{10}^{2}\left(C_{i, e q}-C_{i, w}\right)
$$

where $\gamma_{G}$ is a tunable model parameter of order 1 controlling the magnitude of the flux, $\mathrm{k}_{G}$ is the constant 
used by Wanninkhof (1992) $\left(8.6 \times 10^{-7} \mathrm{~s} \mathrm{~m}^{-1}\right), S c_{i}$ is the Schmidt number (ratio of the kinematic viscosity to the molecular diffusivity of the gas of interest), $u_{10}$ is the wind speed $\left(\mathrm{m} \mathrm{s}^{-1}\right)$ at $10 \mathrm{~m}$ height above sea-surface, and $C_{i, w}$ and $C_{i, e q}$ are the concentrations of gas $i\left(\mathrm{~mol} \mathrm{~m}^{-3}\right)$ in the water and at equilibrium, respectively.

In the model, the flux due to air injection, $F_{A I}$, is determined as the sum of the flux due to bubbles that have been completely trapped, thus injecting air of atmospheric abundances, and those that have been only partially dissolved before rising to the surface, thus resulting in fractionation as a function of diffusivity and solubility (Fuchs et al., 1987; Jenkins, 1988a; Keeling, 1993; Woolf, 1993). The total air injection flux, $F_{A I}$, is equal to

$$
F_{A I}=F_{C}+F_{P}
$$

where $F_{C}$ refers to the flux from bubbles that are completely trapped, and $F_{P}$ refers to the flux from bubbles that are only partially dissolved.

The flux due to completely trapped bubbles is dependent on the partial pressure of the gas in the atmosphere and the volume entrainment rate for the air entrained. This volume entrainment rate is given by a combination of the whitecap coverage formulation of Monahan and Torgersen (1990) which scales as $u_{10}^{3}$ with the air entrainment velocity estimate of Keeling (1993):

$$
F_{C}=A_{c} \cdot k_{w}\left(u_{10}-2.27\right)^{3} \nu_{a} \frac{P_{i, a}}{R T}
$$

where $A_{c}$ is a tunable model parameter that controls the magnitude of the completely trapped bubble flux, $\mathrm{k}_{w}$ is the constant associated with white-cap coverage $\left(1.86 \times 10^{-6} \mathrm{~s}^{3} \mathrm{~m}^{-3}\right), \nu_{a}$ is the air entrainment velocity $\left(0.01 \mathrm{~m} \mathrm{~s}^{-1}\right), R$ is the gas constant $\left(8.31 \mathrm{~J} \mathrm{~mol}^{-1} \mathrm{~K}^{-1}\right), T$ is the temperature $(\mathrm{K})$, and $P_{i, a}$ is the partial pressure of gas $i$ in the atmosphere (Pa). Eq. 4.5 is valid for $u_{10} \geq 2.27 \mathrm{~m} \mathrm{~s}^{-1}$. At $u_{10}<2.27 \mathrm{~m} \mathrm{~s}^{-1}, F_{c}$ is set to 0 .

The partial bubble trapping flux depends upon the same whitecap coverage formulation as in the case of complete trapping, and also on the solubility and diffusivity of the gas, and the difference between the partial pressure of the gas in the bubble and in the water : 


$$
F_{P}=A_{p} \cdot k_{w}\left(u_{10}-2.27\right)^{3} \nu_{a} \alpha_{i} D_{i}^{\frac{2}{3}} \frac{P_{i, b}-P_{i, w}}{R T}
$$

where $A_{p}$ is a tunable model parameter that scales the magnitude of the partial trapping flux, $\alpha$ is the Bunsen solubility coefficient of gas $i, D$ is the diffusivity $\left(\mathrm{m}^{2} \mathrm{~s}^{-1}\right)$ of gas $i$, and $P_{i, b}$ and $P_{i, w}$ are the partial pressures of the gas in the bubble and in the water (Pa). Eq. 4.6 is valid only for $u_{10} \geq 2.27 \mathrm{~m} \mathrm{~s}^{-1}$. At $u_{10}<2.27$ $\mathrm{m} \mathrm{s}^{-1}, F_{p}$ is set to 0 . Eq. 4.6 is similar to the bulk partial trapping flux equation used by Keeling (1993) when appropriate scaling factors and units are used and with the difference that Keeling uses exponents on $\alpha_{i}$ and $D_{i}$ of 0.7 and 0.35 respectively. This is a bulk approximation for partial bubble trapping and does not explicitly take into account bubble trajectories, size spectra, etc. In both warm and cold conditions, $\alpha_{i} D_{i}^{2 / 3}$ varies by approximately a factor of five between the noble gases, being largest for Xe and smallest for $\mathrm{Ne}$.

Calculating the partial pressure of the gas in the bubble, $P_{i, b}$, is a complicated problem of bubble dynamics, since the pressure depends on the size and depth distribution of the bubbles. Here, we simply approximate $P_{i, b}$ by:

$$
P_{i, b}=X_{i}\left(P_{a t m}+\rho g h_{b u b}\right)
$$

where $X_{i}$ is the mole fraction of gas $i$ in dry air, $P_{a t m}$ is the atmospheric pressure of dry air $(\mathrm{Pa}), \rho$ is the density of water $\left(\mathrm{kg} \mathrm{m}^{-3}\right), g$ is the gravitational acceleration $\left(9.81 \mathrm{~m} \mathrm{~s}^{-1}\right)$ and $h_{b u b}(\mathrm{~m})$ is the average dissolution depth for the bubbles. This expression reflects the hydrostatic pressure felt by the bubble but ignores surface tension effects. The depth $h_{b u b}$, used for the hydrostatic pressure calculation, is approximated by:

$$
h_{b u b}=\frac{1}{2}\left(0.3 u_{10}-1.1\right)
$$

which is the bubble cloud residence depth/wind speed relationship of Graham et al. (2004), multiplied by a bubble depth scaling factor of $\frac{1}{2}$ to take into account that the bubbles partially dissolve along their entire downward and upward transect rather than simply at their residence depth. The results were not sensitive to the choice of bubble depth scaling factor since a decrease in the scaling factor could be compensated for by an increase in $A_{p}$. Eq. 4.8 is valid only for $u_{10} \geq 3.7 \mathrm{~m} \mathrm{~s}^{-1}$. At $u_{10}<3.7 \mathrm{~m} \mathrm{~s}^{-1}, h_{b u b}$ is set to 0 . 


\subsubsection{Inverse Method}

The air-sea gas exchange parameters $\gamma_{G}, A_{c}$, and $A_{p}$ were determined inversely by a constrained, non-linear optimization scheme. The parameters determined by the optimization are dependent on the choice of the cost function, and thus we explored several possible formulations of the cost function.

Our "base case" model uses a cost function that includes two components: (1) a contribution from model-data saturation anomalies in the mixed layer and (2) a contribution from model-data concentration difference below the mixed layer. The cost function is equal to

$$
C F=\frac{a_{1}}{N_{\Delta}} \sum_{j=1}^{5}\left(\sum_{i=1}^{N_{\Delta}} \frac{\left(\Delta_{\text {mod }, i, j}-\Delta_{\text {obs }, i, j}\right)^{2}}{\sigma_{\Delta i, j}^{2}}\right)+\frac{a_{2}}{N_{\text {conc }}} \sum_{j=1}^{5}\left(\sum_{i=1}^{N_{\text {conc }}} \frac{\left([X]_{\text {mod }, i, j}-[X]_{\text {obs }, i, j}\right)^{2}}{\sigma_{\text {conc }, i, j}^{2}}\right)
$$

where $a_{1}$ and $a_{2}$ are constants used to make the two components have approximately equal contributions to the cost function, $j$ is a counter for each of the five gases, $i$ is a counter for each of the measurements, $N_{\Delta}$ is the total number of monthly surface saturation anomaly measurements (i.e. 14), $\Delta_{m o d}$ and $\Delta_{o b s}$ are the monthly surface saturation anomalies of the gases in either the model or observations, $\sigma_{\Delta i, j}$ are the uncertainties in the monthly average surface saturation anomaly measurements, $N_{\text {conc }}$ is the total number of concentration measurements, $[X]_{m o d}$ and $[Y]_{\bmod }$ are the concentrations of the gases in either the model or in the observations, and $\sigma_{\text {conc }}$ are the uncertainties in the concentration measurements.

The first component reflects the differences between the model and data's mixed layer depth-averaged monthly surface saturation anomalies. We use average monthly values rather than point-to-point depth comparisons of mixed layer saturation anomaly between our model and data in order to avoid over-emphasizing the winter months (which have more data in the mixed layer simply because the mixed layer is deeper) and to avoid under-emphasizing the summer months, which only have a few data points in the mixed layer since the mixed layer is so shallow. The saturation anomalies are weighted by the combined uncertainty of the sampling collection and analysis uncertainty, the estimated uncertainty in the solubility values for the particular gas, and the standard error of multiple determinations within the mixed layer in the given month, all added in quadrature. The sizes of these contributions to $\sigma_{\Delta}$ for the five noble gases are listed in Table 4.4. The sensitivity of the results to different values of $\sigma_{\Delta}$ is investigated. 
Table 4.4: The magnitudes of the three contributions to $\sigma_{\Delta}$ and the average $\sigma_{\Delta}$ for each of the noble gases (all in \%) . "Meas Error" refers to how well our method can measure the noble gases as determined by duplicate pairs. "Sol Error" refers to estimates of the uncertainty in the solubility determinations of the noble gases. "Std error" refers to the standard error of mean of the multiple measurements within the mixed layer. The standard error and thus $\sigma_{\Delta}$ varies from month to month, with the average value being listed here.

\begin{tabular}{ccccc}
\hline Gas & Meas Error & Sol Error & Std Error & $\sigma_{\Delta}$ \\
\hline $\mathrm{He}$ & 0.96 & 0.5 & 0.40 & 1.2 \\
$\mathrm{Ne}$ & 0.88 & 0.3 & 0.39 & 1.0 \\
$\mathrm{Ar}$ & 0.3 & 0.13 & 0.18 & 0.37 \\
$\mathrm{Kr}$ & 0.29 & 1 & 0.22 & 1.06 \\
$\mathrm{Xe}$ & 0.19 & 1.5 & 0.22 & 1.5 \\
\hline
\end{tabular}

The second component of the cost function is comprised of the difference between the model and data's concentrations below the mixed layer. We use concentrations here, rather than saturation anomalies, because erroneously high (low) temperatures in the model below the mixed layer result in erroneously high (low) saturation anomalies. The model does not achieve the correct thermal structure below the mixed layer; by using concentrations in our cost function, we compare the total inventory of the gases in the water column and thus temperature effects are not as significant. The first and second components of the cost function are scaled using $a_{1}$ and $a_{2}$ so that they contribute in approximately equal proportions. The sensitivity of the results to this scaling is investigated.

We also investigated a cost function that had a third component reflecting the seasonal amplitude of the surface saturation anomaly. Additionally, we explored the sensitivity of our results to a systematic offset in the model-data comparison in order to examine the effect of potential biases in our model, such as those due to our neglect of lateral advection. 


\subsection{Results}

\subsubsection{Noble Gas Data}

Contour plots of the observed and modeled noble gas saturation anomalies (Fig 4-1) and concentrations (Fig 4-2) show distinctive patterns for the noble gases. Helium and neon form one group, with similar saturation anomalies to each other and similar values throughout the upper $160 \mathrm{~m}$. The concentrations of $\mathrm{He}$ are nearly constant throughout the upper $160 \mathrm{~m}$, a reflection of the very weak dependence of $\mathrm{He}$ solubility on temperature. The concentrations of $\mathrm{Ne}$ are slightly higher at deeper depths $-\mathrm{Ne}$ has a weak dependence of solubility on temperature and thus colder water has more Ne. Argon, krypton, and xenon form a second group. These gases show maxima in saturation anomalies in the summer, especially below the mixed layer. In the summer, the water warms, warmer water has a lower solubility, and thus the water becomes supersaturated. Because Xe has the strongest temperature dependence of solubility, the maxima for Xe is the largest. The concentration plots of $\mathrm{Ar}, \mathrm{Kr}$, and $\mathrm{Xe}$ show larger concentrations in colder, deeper water. The large concentrations in the mid-depth water of the spring of 2005 may be a remainder of the large gas concentrations entrained in the winter.

The gases also act as two separate groups in the mixed layer (Fig 4-3). Helium and neon form one group; they have little seasonal variation and are always supersaturated, probably due to air injection. Argon, krypton and xenon form the second group. Their saturation anomalies increase dramatically in the summer. The gases follow the "expected" order with Xe having the largest summer saturation anomaly, and Ar having the smallest summer surface saturation anomaly. Xenon is the most soluble gas with the strongest temperature dependence on solubility, and thus it has the largest summer and the smallest winter surface saturation anomaly. Uncertainties in the solubilities of the noble gases, especially of $\mathrm{Kr}$ and $\mathrm{Xe}$, could cause these surface saturation anomalies to shift to higher or lower values.

Vertical profiles of $\mathrm{He}$ and $\mathrm{Ne}$ (Fig 4-4) reveal that these gases have uniform saturation anomalies with depth. The profiles also reveal considerable scatter in the data. He and $\mathrm{Ne}$ are the most insoluble gases and thus are the most sensitive to contamination from air during sampling and extraction. The variability may be a result of bubbles incorporated during sampling or gas leaking in through the plug valves during storage. Although experiments have shown the gas leaking in should not be a cause for concern, perhaps 
Figure 4-1: Upper ocean saturation anomalies of the five noble gases for the (a) 14 month time-series and for (b) the base case model run. Contours are drawn at $1 \%$ intervals. Please note the different scales for the different gases. The white line denotes the mixed layer depth as determined from bottle temperature analyses of BATS samples using a $\sigma_{\theta}$ criterion of 0.15 . Circles denote the time and depth of samples used in this analysis. Gas exchange parameters used in the base case model are $\gamma_{G}=0.787, A_{c}=3.21 \times 10^{-3}$ and $A_{p}=1.64 \times 10^{5}$.

(a) Data
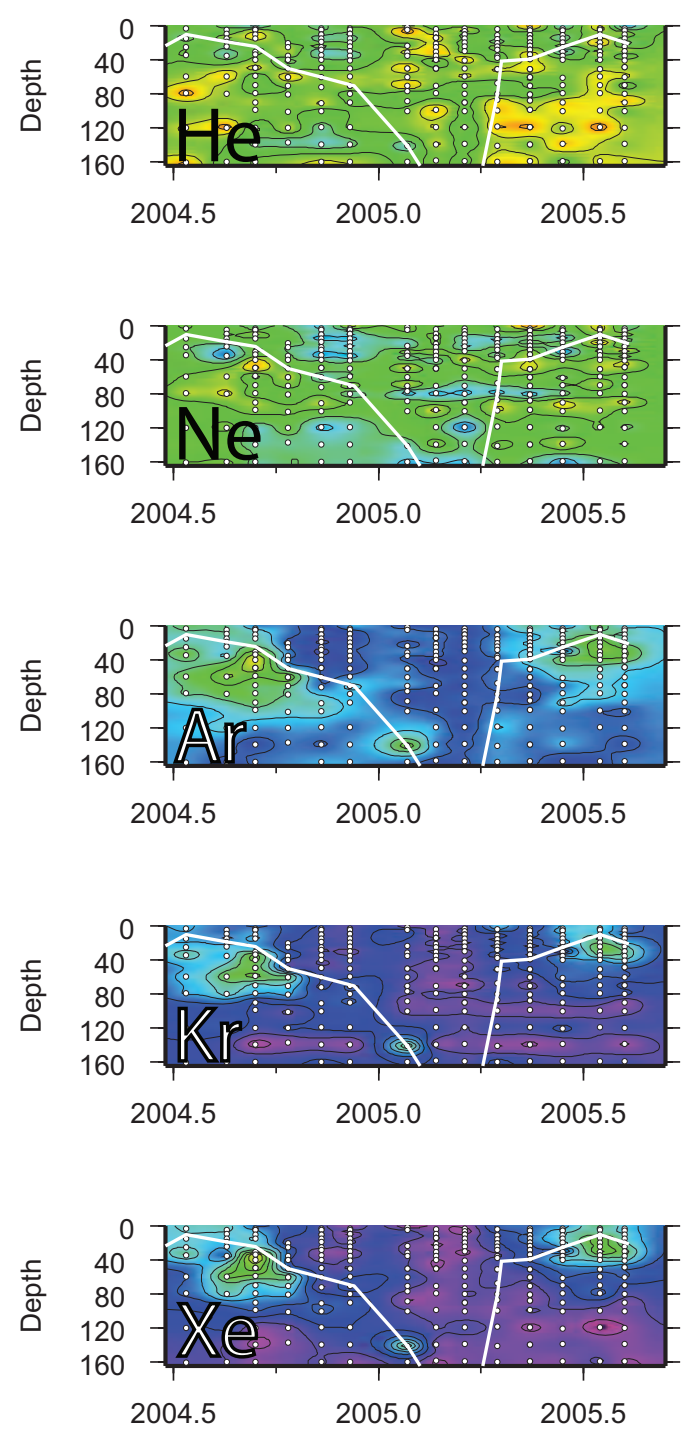

(b) Model
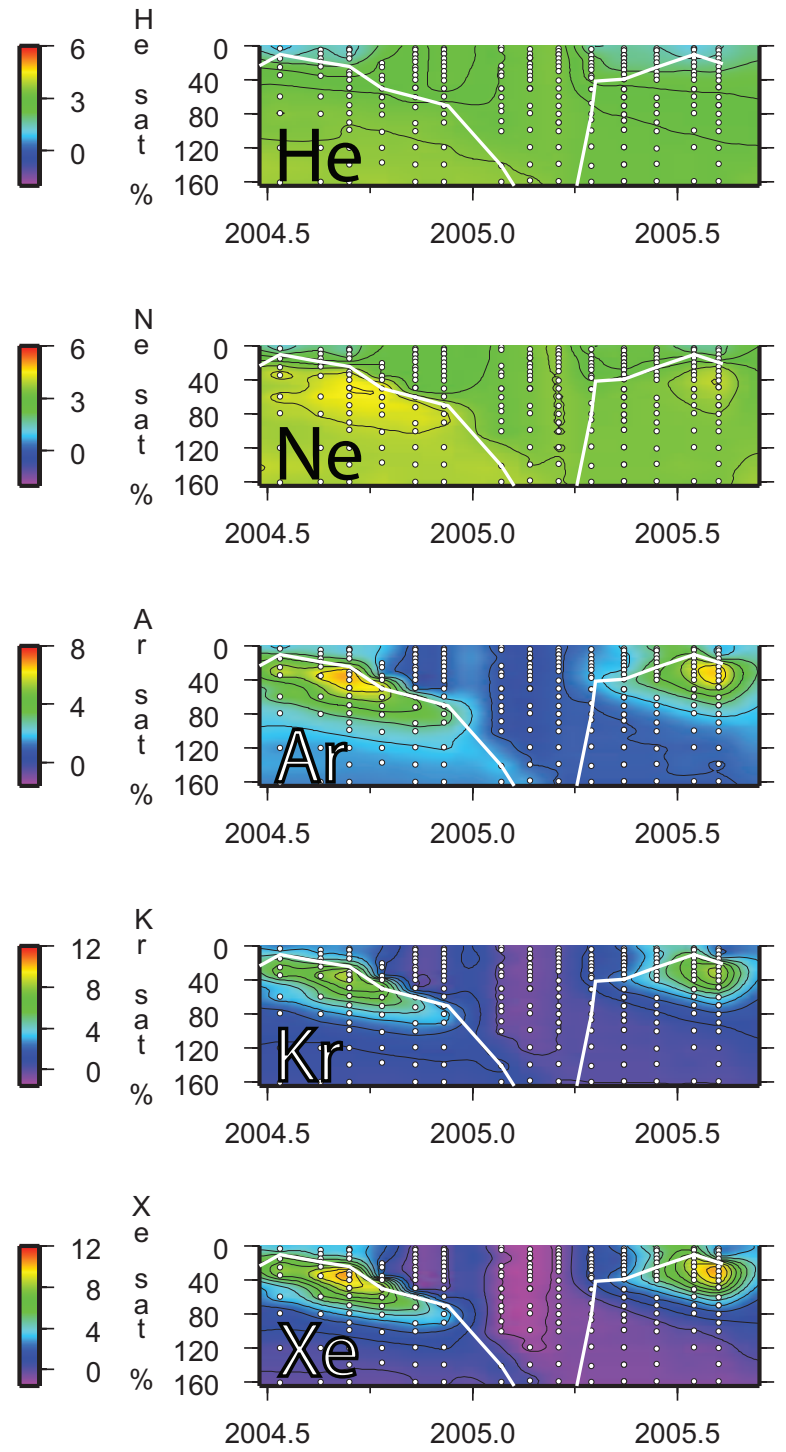
Figure 4-2: Upper ocean concentrations of the five noble gases, normalized to the concentrations of the noble gases in $18^{\circ}$ seawater, for the (a) 14 month time-series and for (b) the base case model run. Contours are drawn at 0.1 intervals. Please note the different scales for the various gases. The white line denotes the mixed layer depth. Circles denote the time and depth of samples used in this analysis. Gas exchange parameters used in the base case model are $\gamma_{G}=0.787, A_{c}=3.21 \times 10^{-3}$ and $A_{p}=1.64 \times 10^{5}$.

(a) Data
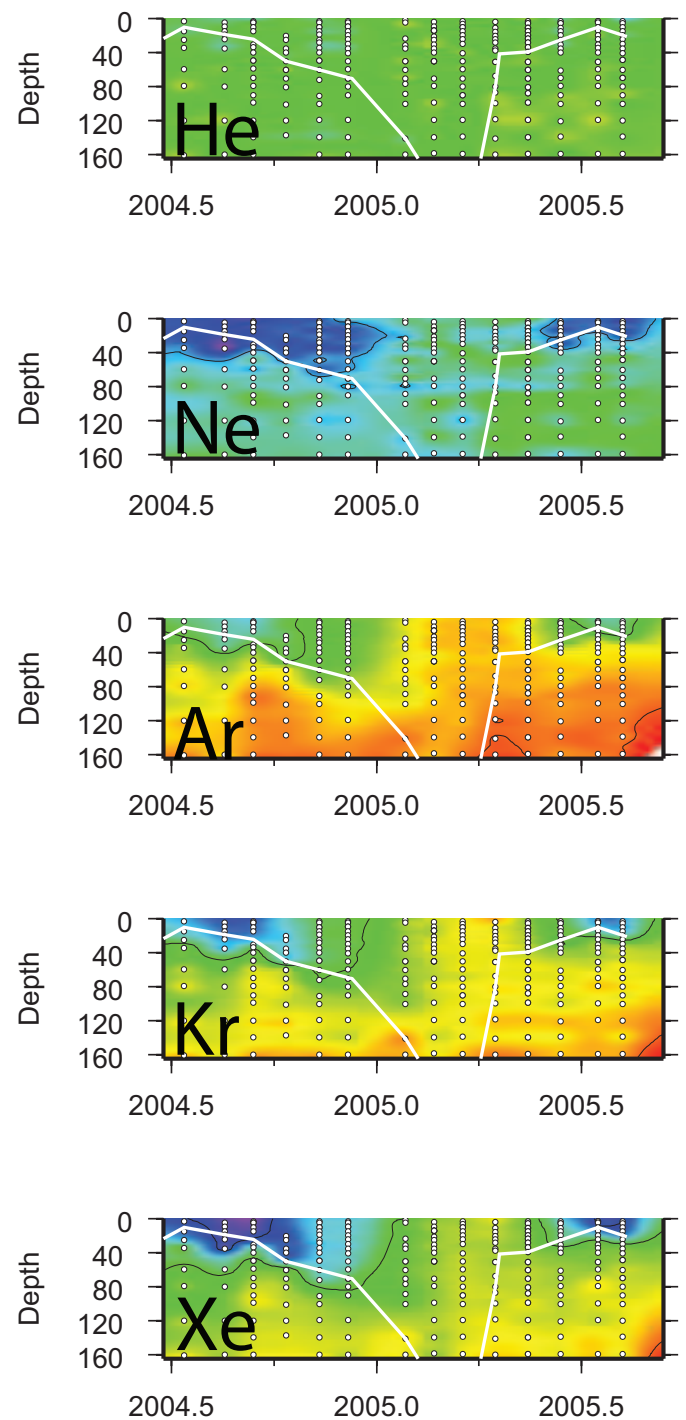

(b) Model
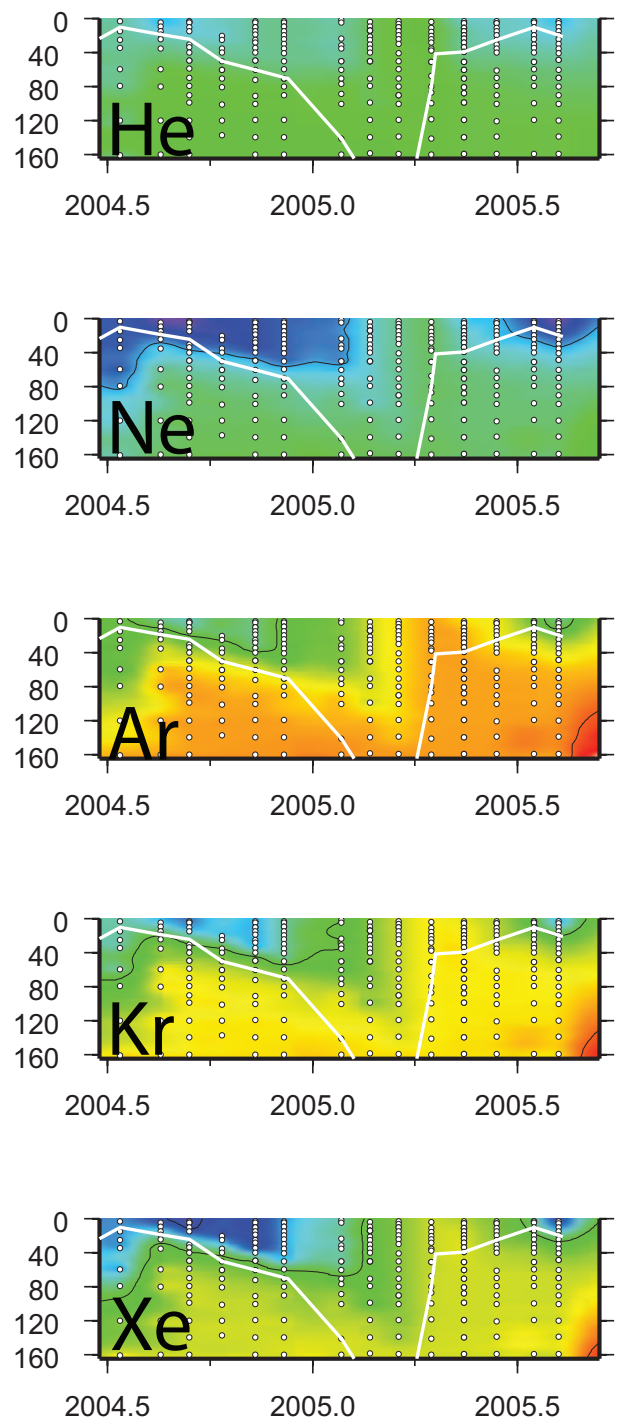
Figure 4-3: Surface saturation anomalies of (a) He (blue) and $\mathrm{Ne}$ (red) and (b) $\mathrm{Ar}$ (blue), $\mathrm{Kr}$ (green), and $\mathrm{Xe}$ (red). Averages of the samples measured in the mixed layer are plotted (o). The error bars reflect the standard error of mean of the measurements in the mixed layer and do not include uncertainties in the solubilities of the noble gases. The model base case is plotted as a solid line. Please note the different scales on the two plots. The data saturation anomalies in the winter of Xe are larger than those predicted by the model and the data saturation anomalies in the summer of 2005 are smaller than those predicted by the model. This discrepancy may be due to errors in the solubilities of Xe (which may depend on temperature) or to problems with the model. The model results here are only plotted at the timepoints at which data was collected and thus the high frequency variability in the model predictions are not shown in this plot. Please see Figure 4-10 for a plot of high frequency variability, which may in particular explain some of the mismatch between the $\mathrm{He}$ and $\mathrm{Ne}$ model results and data.
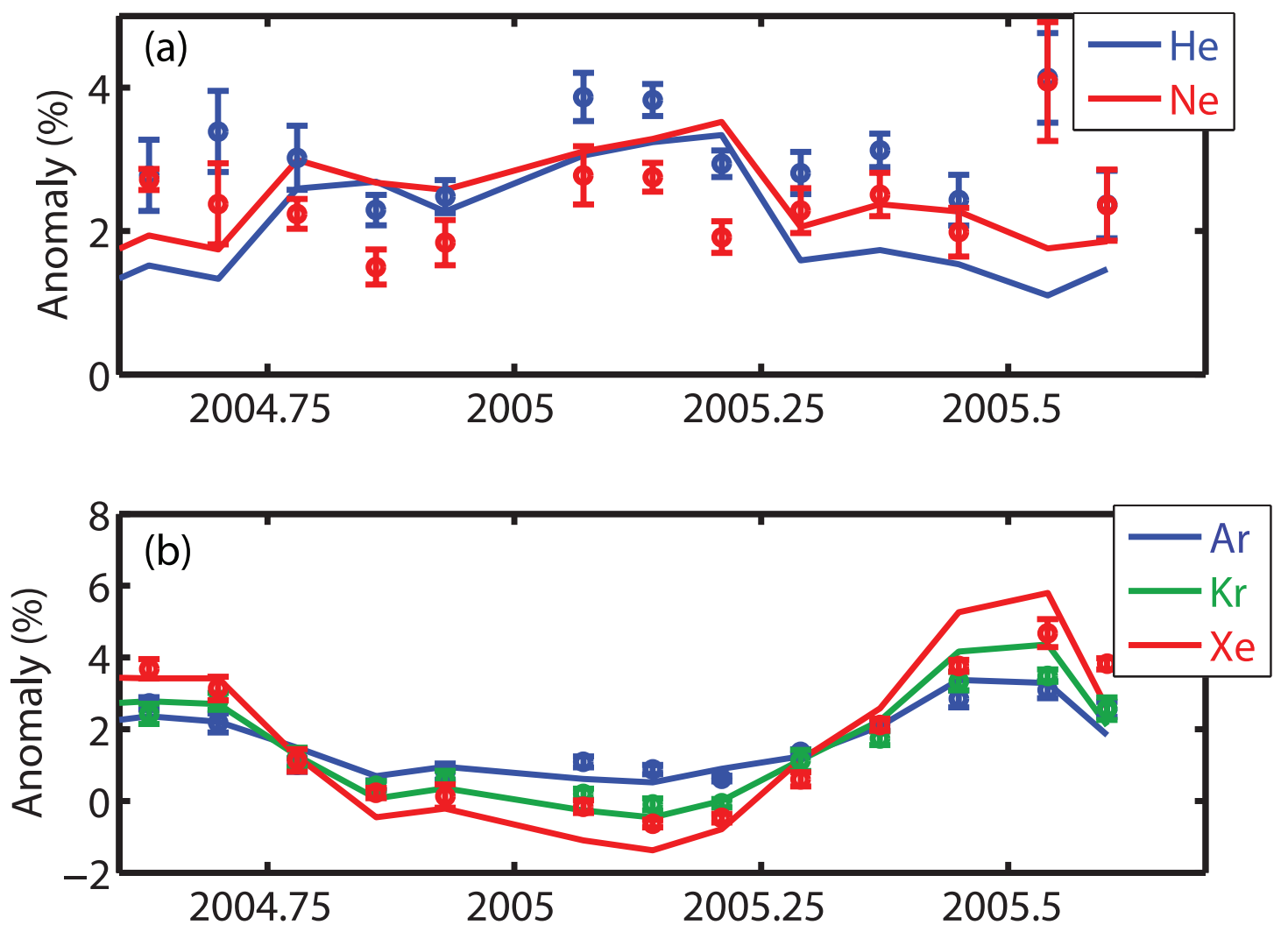
depending on the condition of the o-ring and exactly how it is squeezed when the plug valve is closed, a variable amount of $\mathrm{He}$ and $\mathrm{Ne}$ could be infiltrating the samples. We do not think this variability stems from our analytical method on the mass spectrometer because standards have less than $0.1 \%$ variability.

Winter vertical profiles of $\mathrm{Ar}, \mathrm{Kr}$, and $\mathrm{Xe}$ (Fig 4-5) show uniform saturation anomaly with depth, even below the mixed layer. In contrast, summer profiles show distinct maxima just below the mixed layer, because there the gases cannot "escape" by gas exchange directly. Rather, they need to diffuse into the mixed layer and then, once in contact with the atmosphere, be driven out of the surface ocean. The scatter in the profiles of $\mathrm{Ar}, \mathrm{Kr}$, and $\mathrm{Xe}$ is considerably less than for $\mathrm{He}$ and $\mathrm{Ne}$, supporting the idea that the scatter in $\mathrm{He}$ and $\mathrm{Ne}$ is due to air contamination, a process that would have less of an effect on the heavier, more soluble gases.

\subsubsection{Inverse Model Results}

The gas exchange parameters were determined by the "base case" of the inverse model to be $\gamma_{G}=0.787$, $A_{c}=3.21 \times 10^{-3}$, and $A_{p}=1.64 \times 10^{5}$ (Table 4.5). Since the physical significance of $A_{c}$ and $A_{p}$ is not intuitively obvious, the fluxes of He, Ar, and Xe associated with $A_{c}$ and $A_{p}$ for each of the runs are listed in Table 4.6. Additionally, the complete, partial, and total flux of the sum of all the major gases (i.e. $F_{M G}=$ flux of $\mathrm{O}_{2}$ + flux $\mathrm{N}_{2}+$ flux Ar) are listed in Table 4.7. The base case air injection parameters result in approximately $91 \%$ of the air injection flux of He being due to complete trapping and roughly $70 \%$ of the air injection flux of Xe being due to complete trapping (Table 4.8). Noble gas saturation anomalies and concentrations as determined by the model run with the base case parameters are plotted in Fig 4-1, Fig 4-2, and Fig 4-3.

We explored the sensitivity of the parameters to choice of physical parameters, choice of $\sigma_{\Delta}$ and $\sigma_{\text {conc }}$, and to possible inclusion of seasonal amplitudes in the cost function (Table 4.5). For all these cases, the retrieved value of the gas exchange parameter ranged from 0.74 to $0.84, A_{c}$ from $2.46 \times 10^{-3}$ to $3.43 \times 10^{-3}$, and $A_{p}$ from $1.28 \times 10^{5}$ to $2.87 \times 10^{5}$. The total air injection for the gases varied by $\pm 15 \%$. We also explored the sensitivity of the parameters to potential data/model offsets in noble gas saturation anomalies, perhaps due to lack of inclusion of lateral advection in the model. We tried two choices of constant offsets between model and data surface saturation anomalies: $0.5 \%, 0.75 \%$, and $1 \%$ for $\Delta \mathrm{Ar}, \Delta \mathrm{Kr}$, and $\Delta \mathrm{Xe}$ respectively, and $0.25 \%, 0.375 \%$, and $0.5 \%$ for $\Delta \mathrm{Ar}, \Delta \mathrm{Kr}$ and $\Delta \mathrm{Xe}$ respectively. The relative sizes of these offsets were 
Figure 4-4: Vertical profiles of observed saturation anomalies of $\mathrm{He}$ (blue) and $\mathrm{Ne}$ (red) for twelve months: September 2004 to Aug 2005. The scatter in the profiles reflects the noise in our data - the reproducibility of our $\mathrm{He}$ and $\mathrm{Ne}$ measurements is on order $1 \%$, perhaps because of bubbles trapped during sampling or because of the 24 hour storage time between sample collection and extraction. The dashed line denotes the mixed layer depth.
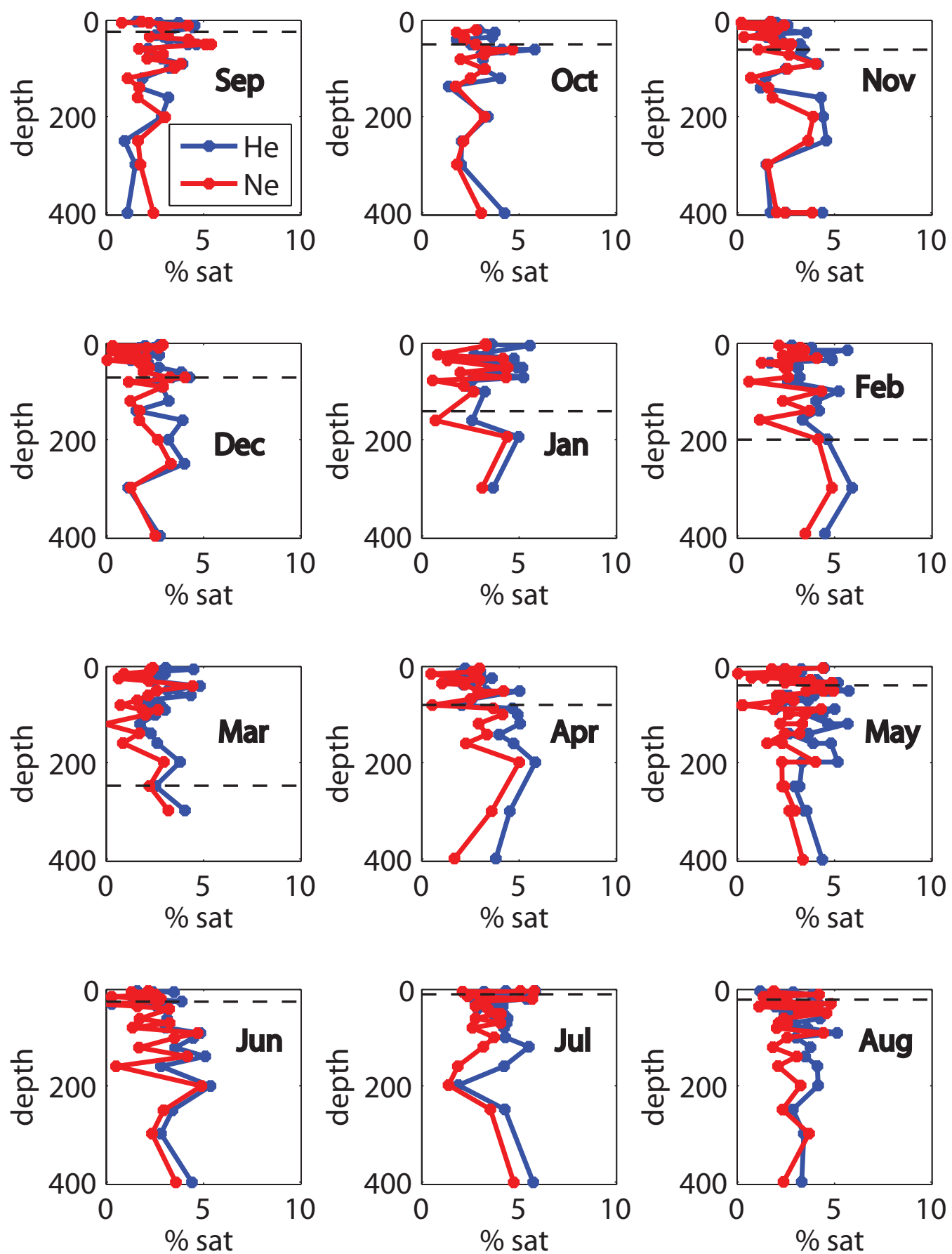
Figure 4-5: Vertical profiles of observed saturation anomalies of $\mathrm{Ar}$ (blue), $\mathrm{Kr}$ (green), and $\mathrm{Xe}$ (red) for twelve months: September 2004 to Aug 2005. The summer months all show a distinctive shape with a maximum in the saturation anomaly just below the mixed layer. The winter months reflect a primarily uniform distribution with depth. The dashed line denotes the mixed layer depth.
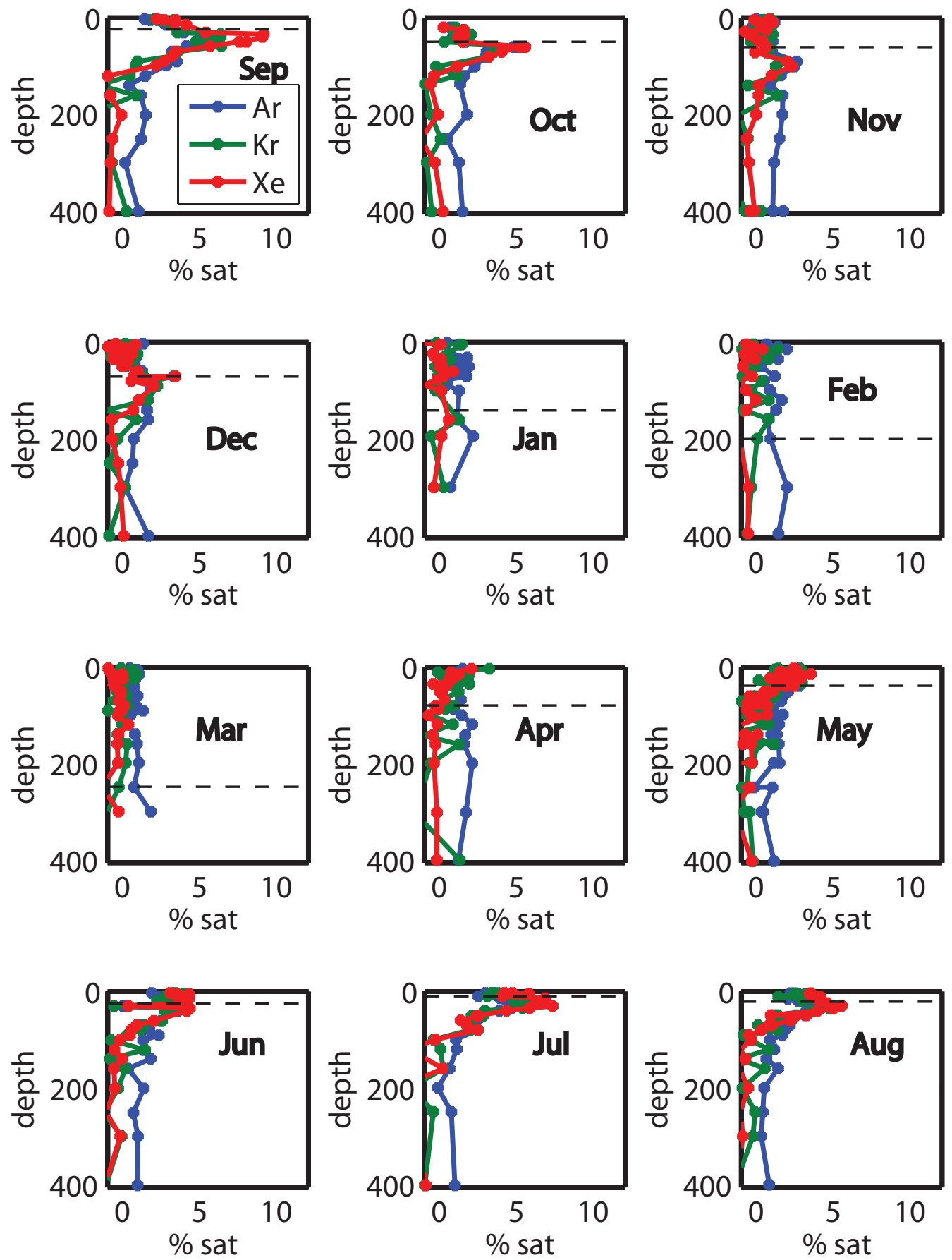
Table 4.5: The air-sea gas exchange parameters as determined by the inverse modeling. The base case is our best estimate for the air-sea gas exchange parameters. In addition, a number of cases are listed and are grouped by the changes in the basic model or cost function. The notes describes in more detail the specific conditions for a given case. Since the parameters $A_{c}$ and $A_{p}$ do not have an intuitively obvious physical significance, estimated size of complete and partial air injection fluxes for $\mathrm{He}$, Ar, and $\mathrm{Xe}$ are listed in Table 4.6. The run numbers allow comparison between the tables.

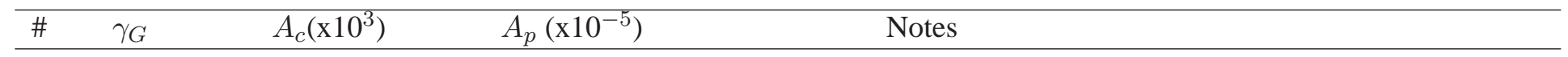

\section{Base Case}

$$
\text { 1. } 0.79
$$

Different Physical Parameters

2. $0.84 \quad 3.43$

3. $0.74 \quad 3.03$

1.66

\section{Different $\sigma_{\Delta}$}

4. $\quad 0.80$

3.22

1.64
5. 0.78

6. 0.82

3.20

3.32

7. 0.79

3.27

Balance between Surface and Deep

$\begin{array}{lll}\text { 8. } & 0.78 & 3.18\end{array}$

9. 0.81

3.28

Including seasonal amplitude

$\begin{array}{lll}\text { 10. } & 0.82 & 2.46 \\ 11 . & 0.80 & 2.90\end{array}$

2.87

2.25

Constant Data/Model Offset
12. 1.02
3.77

13. 0.91

3.85
1.58 Phys Param: $\mathrm{Kz}=6 \times 10^{-5}, \mathrm{Ekm}=20, \mathrm{Z}=50$

Phys Param: $\mathrm{Kz}=4 \times 10^{-5}, \mathrm{Ekm}=16, \mathrm{Z}=50$

1.84 estimated uncertainty of Xe solubility is $2 \%$

1.56 estimated uncertainty of Xe solubility is $1 \%$

1.78 estimated uncertainty of $\mathrm{Kr}$ solubility is $1 \%$

1.28 include in $\sigma_{\Delta}$ the variances of mixed layer sat anom

1.60 surf. sat. anom. weighted twice the deep conc.

1.71 surf. sat. anom. weighted half of the deep conc.

$\mathrm{ml}$ cost contains $50 \%$ absolute mag and 50\% seasonal amp $\mathrm{ml}$ cost contains $75 \%$ absolute mag and $25 \%$ seasonal amp

constant offset of $+0.5 \%,+0.75 \%,+1 \%$ for $\Delta \mathrm{Ar}, \Delta \mathrm{Kr}, \Delta \mathrm{Xe}$ respectively constant offset of $+0.25 \%,+0.375 \%,+0.5 \%$ for $\Delta \mathrm{Ar}, \Delta \mathrm{Kr}, \Delta \mathrm{Xe}$ respectively 
Table 4.6: The fluxes due to complete $\left(F_{C}\right)$ and partial bubble trapping $\left(F_{P}\right)$ in $\mathrm{mol} \mathrm{m}^{-2} \mathrm{~s}^{-1}$ for the runs described in Table 4.5. The run \# allows comparison between the tables. The base case is our best estimate for the air-sea gas exchange parameters. In addition, a number of cases are listed and are grouped by the changes in the basic model or cost function. The notes describes in more detail the specific conditions for a given case. Fluxes were calculated assuming a wind speed of $10 \mathrm{~m} \mathrm{~s}^{-1}$, and saturation anomalies of $1 \%$ for all gases.

\begin{tabular}{|c|c|c|c|c|c|c|c|}
\hline \# & He: $F_{C}$ & He: $F_{P}$ & Ar: $F_{C}$ & Ar: $F_{P}$ & Xe: $F_{C}$ & Xe: $F_{P}$ & Notes \\
\hline \multicolumn{8}{|c|}{ Base Case } \\
\hline 1. & $6.51 \times 10^{-12}$ & $6.64 \times 10^{-13}$ & $1.16 \times 10^{-8}$ & $2.10 \times 10^{-9}$ & $1.08 \times 10^{-13}$ & $4.54 \times 10^{-14}$ & \\
\hline \multicolumn{8}{|c|}{ Different Physical Parameters } \\
\hline 2. & $6.96 \times 10^{-12}$ & $6.42 \times 10^{-13}$ & $1.24 \times 10^{-8}$ & $2.03 \times 10^{-9}$ & $1.16 \times 10^{-13}$ & $4.39 \times 10^{-14}$ & Phys Param: $\mathrm{Kz}=6 \times 10^{-5}, \mathrm{Ekm}=20, \mathrm{Z}=50$ \\
\hline 3. & $6.15 \times 10^{-12}$ & $6.74 \times 10^{-13}$ & $1.10 \times 10^{-8}$ & $2.14 \times 10^{-9}$ & $1.02 \times 10^{-13}$ & $4.61 \times 10^{-14}$ & Phys Param: $\mathrm{Kz}=4 \times 10^{-5}, \mathrm{Ekm}=16, \mathrm{Z}=50$ \\
\hline \multicolumn{8}{|c|}{ Different $\sigma_{\Delta}$} \\
\hline 4. & $6.52 \times 10^{-12}$ & $7.45 \times 10^{-13}$ & $1.16 \times 10^{-8}$ & $2.36 \times 10^{-9}$ & $1.08 \times 10^{-13}$ & $5.10 \times 10^{-14}$ & \multirow{4}{*}{$\begin{array}{l}\text { estimated uncertainty of Xe solubility is } 2 \% \\
\text { estimated uncertainty of Xe solubility is } 1 \% \\
\text { estimated uncertainty of } \mathrm{Kr} \text { solubility is } 1 \% \\
\text { include in } \sigma_{\Delta} \text { the variances of mixed layer sat anom }\end{array}$} \\
\hline 5. & $6.49 \times 10^{-12}$ & $6.33 \times 10^{-13}$ & $1.16 \times 10^{-8}$ & $2.01 \times 10^{-9}$ & $1.08 \times 10^{-13}$ & $4.33 \times 10^{-14}$ & \\
\hline 6. & $6.73 \times 10^{-12}$ & $7.23 \times 10^{-13}$ & $1.20 \times 10^{-8}$ & $2.29 \times 10^{-9}$ & $1.12 \times 10^{-13}$ & $4.94 \times 10^{-14}$ & \\
\hline 7. & $6.64 \times 10^{-12}$ & $5.21 \times 10^{-13}$ & $1.18 \times 10^{-8}$ & $1.65 \times 10^{-9}$ & $1.10 \times 10^{-13}$ & $3.57 \times 10^{-14}$ & \\
\hline \multicolumn{8}{|c|}{ Balance between Surface and Deep } \\
\hline 9. & $6.65 \times 10^{-12}$ & $6.95 \times 10^{-13}$ & $1.19 \times 10^{-8}$ & $2.20 \times 10^{-9}$ & $1.10 \times 10^{-13}$ & $4.75 \times 10^{-14}$ & $\begin{array}{l}\text { surf. sat. anom. weighted twice the deep conc. } \\
\text { surf. sat. anom. weighted half of the deep conc. }\end{array}$ \\
\hline \multicolumn{8}{|c|}{ Including seasonal amplitude } \\
\hline 10. & $4.99 \times 10^{-12}$ & $1.17 \times 10^{-12}$ & 8.89E-09 & $3.69 \times 10^{-9}$ & $8.28 \times 10^{-14}$ & $7.97 \times 10^{-14}$ & \multirow{2}{*}{$\begin{array}{l}\mathrm{ml} \text { cost contains } 50 \% \text { absolute mag and } 50 \% \text { seasonal amp } \\
\mathrm{ml} \text { cost contains } 75 \% \text { absolute mag and } 25 \% \text { seasonal amp }\end{array}$} \\
\hline 11. & $5.88 \times 10^{-12}$ & $9.11 \times 10^{-13}$ & $1.05 \times 10^{-8}$ & $2.89 \times 10^{-9}$ & $9.76 \times 10^{-14}$ & $6.23 \times 10^{-14}$ & \\
\hline \multicolumn{8}{|c|}{ Constant Data/Model Offset } \\
\hline 12. & $7.63 \times 10^{-12}$ & 0 & $1.36 \times 10^{-8}$ & 0 & $1.27 \times 10^{-13}$ & 0 & \multirow{2}{*}{$\begin{array}{l}\text { offset of }+0.5 \%,+0.75 \%,+1 \%, \text { for } \Delta \mathrm{Ar}, \Delta \mathrm{Kr}, \Delta \mathrm{Xe} \\
\text { offset of }+0.25 \%,+0.375 \%,+0.5 \% \text { for } \Delta \mathrm{Ar}, \Delta \mathrm{Kr}, \Delta \mathrm{Xe}\end{array}$} \\
\hline 13. & $7.80 \times 10^{-12}$ & 0 & $1.39 \times 10^{-8}$ & 0 & $1.30 \times 10^{-13}$ & 0 & \\
\hline
\end{tabular}


Table 4.7: The air injection fluxes of the sum of the major gases, $F_{M G}$, due to complete and partial bubble trapping, as well as due to total air injection in mol m $\mathrm{m}^{-2} \mathrm{~s}^{-1}$ for the runs described in Table 4.5. The run \# allows comparison between the tables. The base case is our best estimate for the air-sea gas exchange parameters. In addition, a number of cases are listed and are grouped by the changes in the basic model or cost function. The notes describes in more detail the specific conditions for a given case. Fluxes were calculated assuming a wind speed of $10 \mathrm{~m} \mathrm{~s}^{-1}$, and saturation anomalies of $1 \%$ for all gases.

\begin{tabular}{|c|c|c|c|c|}
\hline \# & Complete $F_{M G}$ & Partial $F_{M G}$ & Total $F_{M G}$ & Notes \\
\hline \multicolumn{5}{|c|}{ Base Case } \\
\hline 1 & $1.23 \times 10^{-6}$ & $1.39 \times 10^{-7}$ & $1.37 \times 10^{-6}$ & \\
\hline \multicolumn{5}{|c|}{ Different Physical Parameters } \\
\hline 2 & $1.31 \times 10^{-6}$ & $1.34 \times 10^{-7}$ & $1.45 \times 10^{-6}$ & Phys Param: $K z=6 E-5, E k m=20, Z=50$ \\
\hline 3 & $1.16 \times 10^{-6}$ & $1.41 \mathrm{E}-07$ & $1.30 \times 10^{-6}$ & Phys Param: $\mathrm{Kz}=4 \mathrm{E}-5, \mathrm{Ekm}=16, \mathrm{Z}=50$ \\
\hline \multicolumn{5}{|c|}{ Different $\sigma_{\Delta}$} \\
\hline 4 & $1.23 \times 10^{-6}$ & $1.56 \times 10^{-7}$ & $1.39 \times 10^{-6}$ & estimated uncertainty of Xe solubility is $2 \%$ \\
\hline 5 & $1.23 \times 10^{-6}$ & $1.33 \times 10^{-7}$ & $1.36 \times 10^{-6}$ & estimated uncertainty of Xe solubility is $1 \%$ \\
\hline 6 & $1.27 \times 10^{-6}$ & $1.51 \times 10^{-7}$ & $1.42 \times 10^{-6}$ & estimated uncertainty of $\mathrm{Kr}$ solubility is $1 \%$ \\
\hline 7 & $1.25 \times 10^{-6}$ & $1.09 \times 10^{-7}$ & $1.36 \times 10^{-6}$ & include in sigma the variances of mixed layer sat anom \\
\hline \multicolumn{5}{|c|}{ Balance between Surface and Deep } \\
\hline 8 & $1.22 \times 10^{-6}$ & $1.36 \times 10^{-7}$ & $1.35 \times 10^{-6}$ & surf. sat. anom. weighted twice the deep conc. \\
\hline 9 & $1.26 \times 10^{-6}$ & $1.46 \times 10^{-7}$ & $1.40 \times 10^{-6}$ & surf. sat. anom. weighted half of the deep conc. \\
\hline \multicolumn{5}{|c|}{ Including seasonal amplitude } \\
\hline 10 & $9.41 \times 10^{-6}$ & $2.44 \times 10^{-7}$ & $1.19 \times 10^{-6}$ & $\mathrm{ml}$ cost contains $50 \%$ absolute mag and $50 \%$ seasonal amp \\
\hline 11 & $1.11 \times 10^{-6}$ & $1.91 \times 10^{-7}$ & $1.30 \times 10^{-6}$ & $\mathrm{ml}$ cost contains $75 \%$ absolute mag and $25 \%$ seasonal amp \\
\hline \multicolumn{5}{|c|}{ Constant Data/Model Offset } \\
\hline 12 & $1.44 \times 10^{-6}$ & 0 & $1.44 \times 10^{-6}$ & constant offset of $+0.5 \%,+0.75 \%,+1 \%$ for $\Delta \mathrm{Ar}, \Delta \mathrm{Kr}, \Delta \mathrm{Xe}$ respectively \\
\hline 13 & $1.44 \times 10^{-6}$ & 0 & $1.44 \times 10^{-6}$ & constant offset of $+0.25 \%,+0.375 \%,+0.5 \%$ for $\Delta \mathrm{Ar}, \Delta \mathrm{Kr}, \Delta \mathrm{Xe}$ respectively \\
\hline
\end{tabular}


Table 4.8: The percentages of complete and partial bubble trapping with respect to the total air injection flux for a given gas for the five noble gases and for $\mathrm{O}_{2}$ for the "base case" parameters. Fluxes were calculated assuming a wind speed of $10 \mathrm{~m} \mathrm{~s}^{-1}$, and saturation anomalies of $1 \%$ for all gases.

\begin{tabular}{ccc}
\hline Gas & Complete (\%) & Partial(\%) \\
\hline $\mathrm{He}$ & 91 & 9 \\
$\mathrm{Ne}$ & 93 & 7 \\
$\mathrm{Ar}$ & 85 & 15 \\
$\mathrm{Kr}$ & 79 & 21 \\
$\mathrm{Xe}$ & 70 & 30 \\
$\mathrm{O}_{2}$ & 86 & 14 \\
\hline
\end{tabular}

chosen by comparing the temperature dependence of the solubility value of the gases. The gas exchange parameters differed by up to $30 \%$ for the larger offsets, suggesting that uncertainties in our physical model propagate into uncertainties in the gas exchange parameters.

\subsection{Discussion}

\subsubsection{The Base Case}

It is evident that the model base case captures the general features of the data. The saturation anomalies in the model and the data (Fig. 4-1) both show distinctive maxima in the summer seasonal thermocline for the heavier noble gases, especially below the mixed layer. The general location in time of these maxima agree in the model and the data but the location in space differs. The maxima in the model for $\mathrm{Ar}, \mathrm{Kr}$, and $\mathrm{Xe}$ extend deeper than in the data. This may be due to temperature effects. The saturation anomaly is directly related to the temperature of the data, since the solubility of the heavier gases has a strong temperature dependence. One issue is the presence in the data of eddies which cannot be captured in a one-dimensional model (Fig. 4-6). In August, 2004 an anticyclonic "warm-core" eddy passed through BATS and the model temperature is too cool by over $2{ }^{\circ} \mathrm{C}$. In August 2005, a cyclonic "cool-core" eddy passed through BATS and 
the model temperature is too warm by approximately $2^{\circ} \mathrm{C}$. A one degree change in temperature corresponds to a $3 \%$ difference in Xe saturation anomaly and thus it is not surprising that the model has trouble matching the saturation anomaly structure below the mixed layer. Contours of the difference between the model and data saturation anomalies (Fig. 4-7a) clearly show that the model has too large a saturation anomaly during the August 2005 warm eddy event. Additionally, the model may have trouble matching the temperature record because the model attenuates radiation according to a type Jerlov 1A relationship that does not vary according to season. If desired, however, one could use satellite observations of cholorophyll to modulate the penetration of solar radiation.

However, the discrepancies in saturation anomalies between model and data are not solely due to the temperature effects from eddies because the model also has too large saturation anomalies just below the mixed layer in the fall of 2004. The model predicts larger saturation anomalies than the data even in August 2004 when the model is too cool (and thus when the temperature discrepancy would lead to the model having lower saturation anomalies). Furthermore, contour maps of the concentration anomaly between model and data (Fig. 4-7b) also show the same pattern with the model having too much gas below the mixed layer in the fall of 2004. One possible explanation may that the mismatch is related to the formation mechanisms for the eddies that passed through the BATS site.

Although the He and Ne data are noisy, the model does a good job at predicting the mean general pattern of the saturation anomalies of $\mathrm{He}$ and $\mathrm{Ne}$ - perhaps because they are less dependent on thermal structure. Please note the scales for He and Ne model/data differences in saturation anomaly in Fig. 4-1 are half the size of those for the heavier noble gases. The model predicts too large a saturation anomaly for $\mathrm{Ne}$ and too small a one for He. This inability of the model to match the He/Ne ratio in the data may be a reflection of uncertainties in the solubility of $\mathrm{He}$, inadequacies in the formulation of air injection, or problems in sample collection or analysis. The fact that a number of our He samples had to be discarded because of bubbles presents the possibility that all the samples may have low level air injection contamination. We tried to correct for this using the results of our distilled water tests (see section 4.2), but we may not have fully accounted for what would presumably be a variable process.

In the mixed layer (Fig. 4-3), the model captures roughly the correct magnitude of $\mathrm{He}$ and $\mathrm{Ne}$ saturation but again does not correctly reproduce the ratio between the gases. In the model, $\mathrm{Ne}$ is at the same saturation 
Figure 4-6: Contour maps of the temperature (in ${ }^{\circ} \mathrm{C}$ ) in the upper ocean from (a) BATS data and (b) Model predictions. (c) The difference between the model and data reveals that the model is too cool in August 2004 when an anticyclonic eddy resulted in warmer than typical temperatures at BATS. The model is too warm in August 2005 when a cyclonic eddy resulted in cooler than typical temperatures at BATS. At other times, the model and data temperature agree to within $1^{\circ} \mathrm{C}$. The white line denotes the mixed layer depth. Circles denote the time and depth of samples used in this analysis. Physical parameters used in this model run are $K_{z}=5 \times 10^{-5} \mathrm{~m}^{2} \mathrm{~s}^{-1}, \mathrm{Ekm}=18 \mathrm{~W} \mathrm{~m}^{-2}$, and $Z=50 \mathrm{~m}$.

(a) BATS temperature (Data)

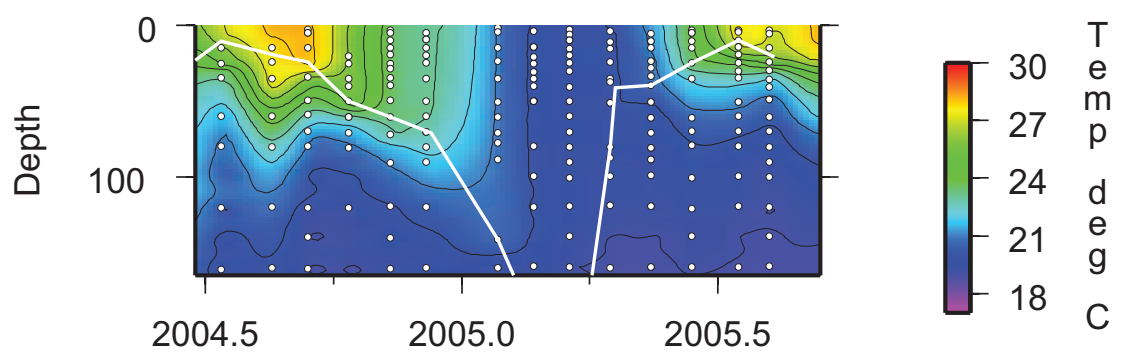

(b) Model Temperature

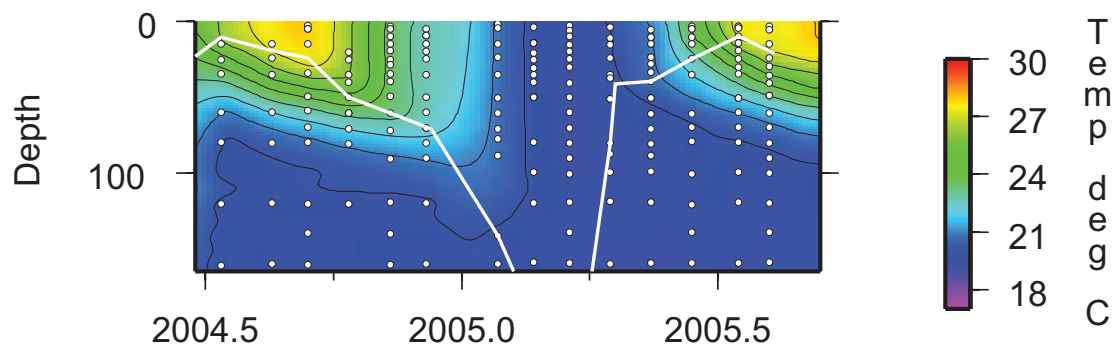

(c) Temperature Anomaly: Model - Data

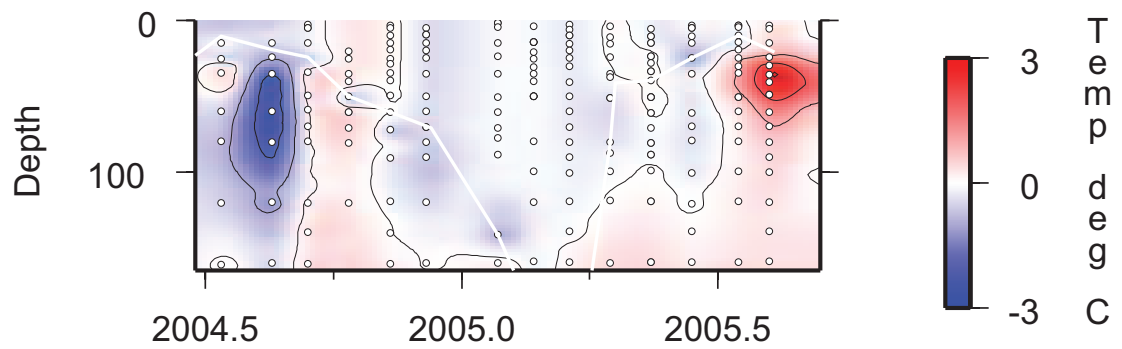


Figure 4-7: (a) Model - data differences of saturation anomalies. The saturation anomaly differences are plotted in \%. Contour lines are every $1 \%$. (b) Model - Data differences of concentrations. The concentrations were normalized first to 18 degree water and then the differences in these normalized concentrations are plotted. Contour lines are drawn at 0.05 intervals. In the summer of 2005, there is a large positive saturation anomaly difference between model and data but only a small concentration difference, reflecting that the saturation anomaly differences are also dependent on the temperature differences between model and data. We use a cost function with saturation anomalies in the mixed layer and concentrations at depth in order to try to minimize the effect of mismatches in temperature between model and data below the mixed layer.

\section{(a) Saturation Anomalies Model-Data Differences}
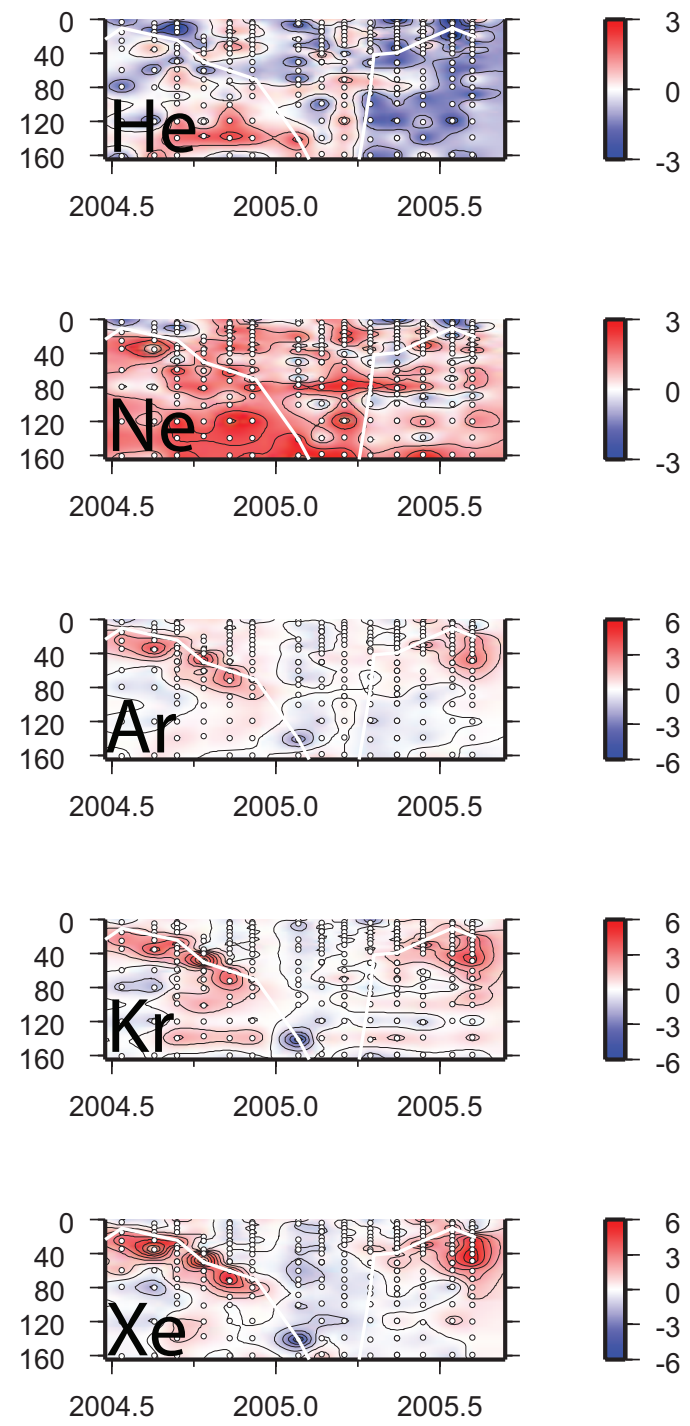

(b) Concentration Model-Data Differences
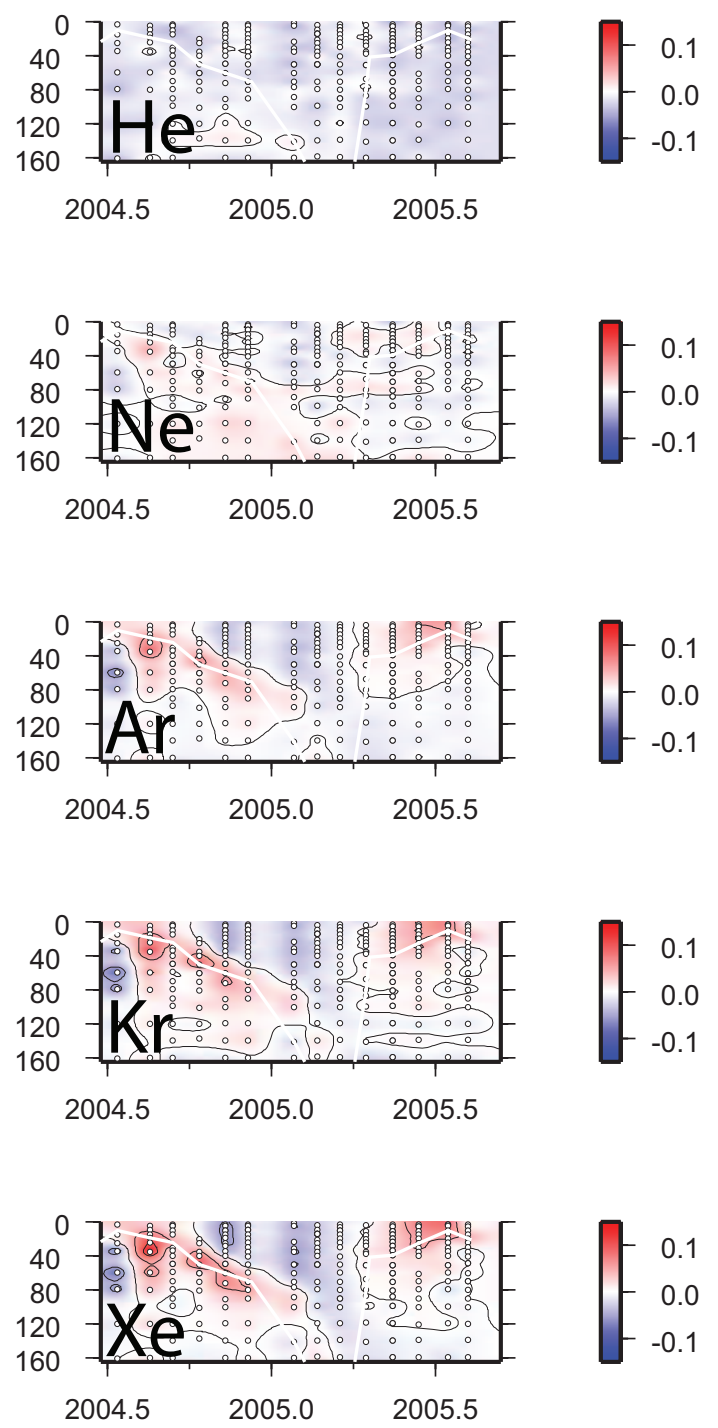
level or even more saturated than He, whereas in the data the reverse is true. Again, this may be because contamination from air is causing the variability in our He results and air contamination affects He more strongly than Ne. The model does a good job at matching the Ar, Kr, and Xe saturation anomalies in the summer of 2004. Unfortunately, it does not do as good a job in the summer of 2005, which may be the more important summer to match since we have data from the winter preceding the summer of 2005 . Future work should involve changing the weightings so that the data in the summer of 2004 contributes less to the cost function than the data in the summer of 2005. Additionally, the model and data in the winter time are within errors of each other for Ar and $\mathrm{Kr}$. However, the data is about $1 \%$ larger than the model predicts for Xe. This discrepency for Xe suggests either errors in the solubility functions of Xe, or that the model may not be incorporating all the processes that in the ocean affect the fluxes of these gases, such as lateral advection. eding the summer of 2005. Future work should involve changing the weightings so that the data in the summer of 2004 contributes less to the cost function than the data in the summer of 2005. Additionally, the model and data in the winter time are within errors of each other for Ar and $\mathrm{Kr}$. However, the data is about $1 \%$ larger than the model predicts for Xe. This discrepency for Xe suggests either errors in the solubility functions of Xe, or that the model may not be incorporating all the processes that in the ocean affect the fluxes of these gases, such as lateral advection.

Our model does not explicitly include lateral processes. We try to compensate for lateral heat advection by adding in a heating compensation term. We do not make any corrections for lateral transport of the gases. In this region, warm water flows northward, resulting in a net cooling. Since warm water has lower gas concentrations, if the gases are at equilibrium, then water with low gas concentrations flows northward, increasing in gas, and resulting in a net influx of gas across the air-sea interface. We do not account for this possible process in our model, partially because we do not know if the gases are at equilibrium. It is possible the gases do not fully equilibrate and thus the water that flows "out" of our region is still low in gas and is undersaturated. We can roughly estimate the maximum size of such a lateral effect by multiplying the solubility dependence on temperature of the noble gases with the change in temperature that is caused by our heating compensation term. The size of this estimated flux (Fig. 4-8) is small compared to the sizes of the diffusive gas exchange and air injection fluxes for all the gases except for $\mathrm{Kr}$ and especially $\mathrm{Xe}$, suggesting that it may not be adding much error for the gases apart from $\mathrm{Kr}$ or $\mathrm{Xe}$. 
Figure 4-8: Integrated monthly fluxes of the noble gases (in $\mathrm{mol} \mathrm{m}^{-2} \mathrm{y}^{-1}$ ) as calculated by the model for the five noble gases. The three types of air-sea gas exchange fluxes - that due to diffusive gas exchange (blue), complete air injection (green), and partial air injection (red) - are dominant. Also shown is the flux of gases associated with Ekman pumping (cyan) and the estimated gas flux associated with the model's heat compensation (purple). This last flux is not added explicitly in the model and is shown here for reference only.
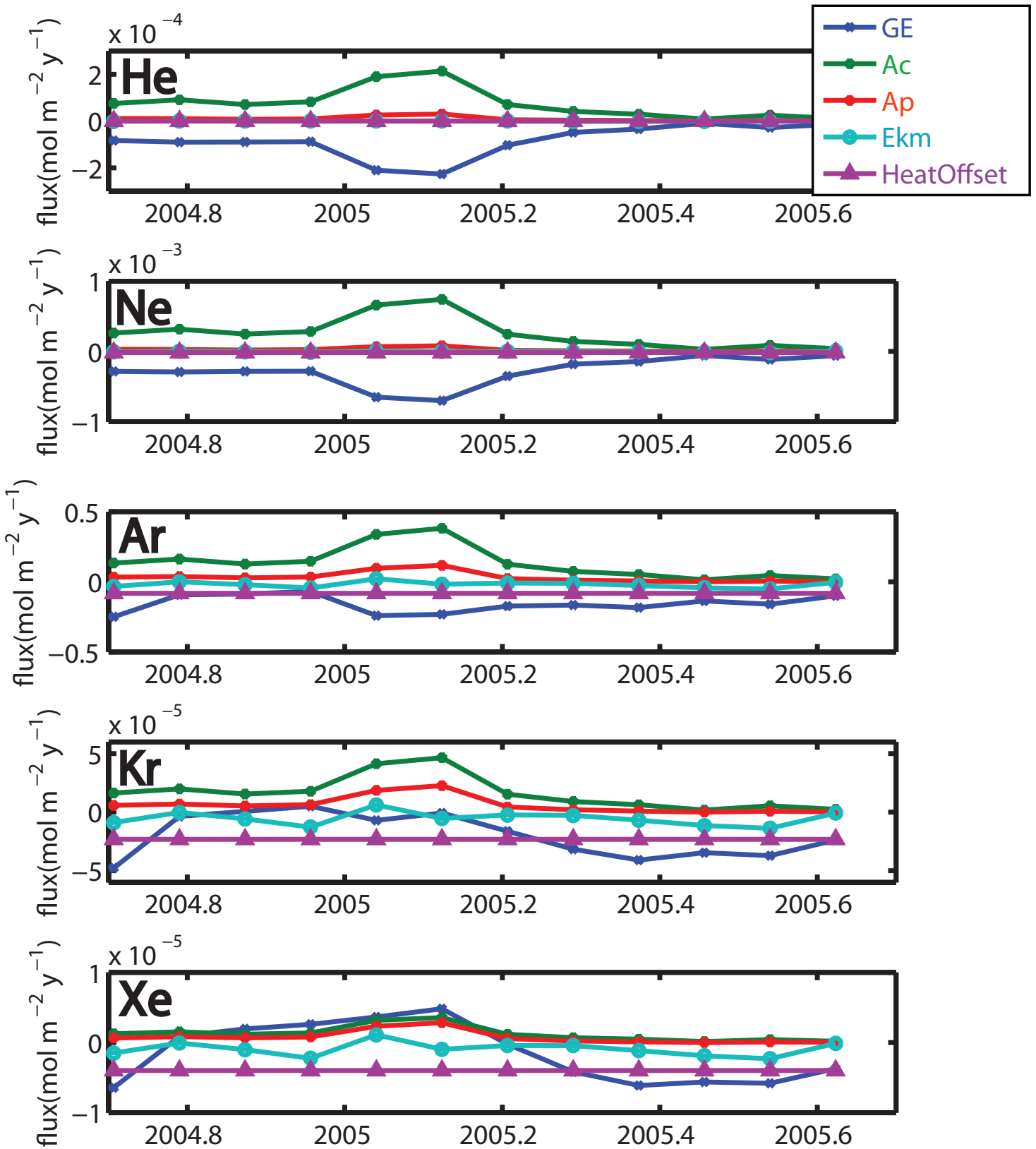
In Figure 4-8, we plot the integrated monthly fluxes predicted by the model for diffusive gas exchange, and partial and complete air injection. For $\mathrm{He}$ and $\mathrm{Ne}$, the air injection flux into the ocean is balanced by the diffusive gas exchange flux out of the ocean. For Ar, Kr, and Xe, there is a diffusive gas exchange flux out of the ocean in the summer months due to thermal warming. This flux is independent of the air injection - the separation between air injection and diffusive gas exchange for these heavier gases allows us to constrain both air injection and diffusive gas exchange. In the winter, however, even the signatures of the heavier gases reflect the interacting processes of air injection and diffusive gas exchange. For Xe, in the winter there is a diffusive gas exchange flux and a significant air injection flux into the ocean, with the winter total air injection flux being larger than the diffusive one. For $\mathrm{Ar}$ and $\mathrm{Kr}$, in the winter, air injection brings in enough gas that the diffusive gas exchange flux drives gas out of the ocean, in spite of the cooling of the water. Also plotted on the figure is an estimate of the size of the flux of gas due to Ekman pumping and of the possible gas flux associated with the heating compensation flux added to our model. For $\mathrm{He}, \mathrm{Ne}$, and Ar these fluxes are both small compared to the gas exchange fluxes, suggesting uncertainties in these fluxes do not add much error to our estimates of gas exchange flux. For $\mathrm{Kr}$ and $\mathrm{Xe}$, however, the potential flux associated with the heating compensation flux added to the model is of similar size to the gas exchange fluxes, suggesting that the lack of including an associated gas flux in the model with the heating compensation term may be problematic.

The magnitude of the fluxes seems reasonable when compared to the monthly changes in gas inventories in the top $160 \mathrm{~m}$ of the water column (Fig. 4-9). The inventories were calculated by fitting a spline to the individual monthly profiles of model or data concentrations of the noble gases and then integrating the spline over the top $160 \mathrm{~m}$ of the water column. The inventory of gases in the model and data agree within $2 \%$ for $\mathrm{He}, 0.5 \%$ for $\mathrm{Ne}$ and $\mathrm{Ar}$, and $1 \%$ for $\mathrm{Kr}$ and $\mathrm{Xe}$. Helium is consisently higher in the data than in the model, perhaps due to air contamination issues or to errors in solubility. The inventories of the other gases do not show systematic offset between the data and the model. Since one of the terms of our cost function (Eq. 4.9) was the concentration of the gases, it is not surprising that the inventories agree well. Nonetheless, it is gratifying that the model and the data are able to so closely match inventories, reinforcing the idea that the discrepencies in saturation anomaly between the model and data are primarily a result of the temperature discrepencies between model and data. 
Figure 4-9: Inventories of the noble gases (in $\mathrm{mol} \mathrm{m}^{-2}$ ) in the upper $160 \mathrm{~m}$ in the base case model (dashed line) and from the data (solid line) were calculated every month by integrating the concentration profiles. The model and data agree within $2 \%$ for He with the model consistently underestimating the inventory. There is not a consistent discrepency for the other gases, and the model and data agree to within $0.5 \%$ for $\mathrm{Ne}$ and $\mathrm{Ar}$, and to within $1 \%$ for $\mathrm{Kr}$ and $\mathrm{Xe}$.
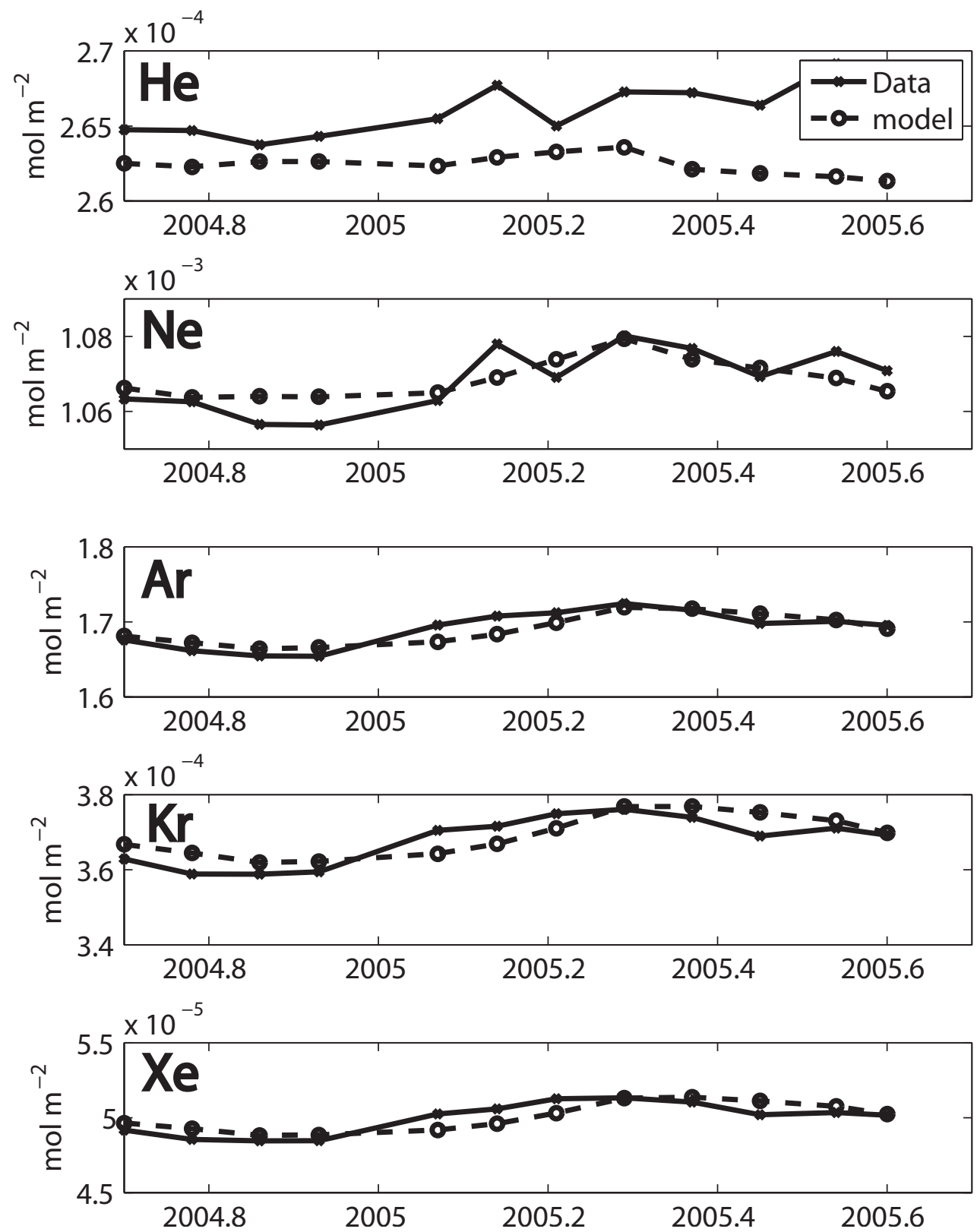
Our model does not include the cool skin effect. In the ocean, a thin layer $(\sim 1 \mathrm{~mm})$ at the surface has a cooler temperature of $\sim 0.2$ to $0.3{ }^{\circ} \mathrm{C}$ due to evaporative and radiative cooling (Fairall et al., 1996; Wick et al., 2005). Since Xe is more soluble at colder temperatures, the effective saturation anomaly of Xe at the surface is $\sim 0.5$ to $0.9 \%$ smaller than calculated in our model. The magnitude of the diffusive gas exchange flux would thus be larger than the model predicts when Xe is undersaturated and smaller than the model predicts when Xe is supersaturated. Future versions of the model should address this cool skin effect. However, recent estimates of the cool skin effect on the gas exchange of $\mathrm{CO}_{2}$ suggest it is less important than previously had been thought (McGillicuddy et al., 2007; Zhang and Cai, 2007), in part because the temperature boundary layer is an order of magnitude thicker than the mass boundary layer.

\subsubsection{Controls on the Parameters}

In order to assess which gases are controlling the values of the parameters, we performed a "knock-out" study where we systematically increased $\sigma_{\Delta}$ to 1000 (from the typical value of 1 ) for each gas in turn (Table 4.9). We thus examined how the parameters changed depending on which gases were included in the cost function. If the parameters did not change much after the exclusion of a given gas, then that gas is not exerting much control on the parameter. The diffusive gas exchange parameter, $\gamma_{G}$ changed by at most $10 \%$ if any one gas was excluded from the cost function. Hence, all the gases suggest similar values for $\gamma_{G}$ and thus our value of 0.79 is robust to choice of gases included. This gives us confidence in at least the consistency of our results.

The total air injection flux changes by up to $25 \%$ if one of the gases is excluded. The largest total air injection flux is predicted when $\mathrm{Ne}$ is excluded. Once again, $\mathrm{He}$ and $\mathrm{Ne}$ are playing off each other to some extent with the He data pointing to a larger air injection flux and the Ne data pointing to a smaller air injection flux. The fraction of complete vs. partial air injection changes dramatically depending on which gases are included in the cost function - from the extreme case of being all complete trapping if $\mathrm{Ne}$ is excluded to to being predominantly partial trapping if $\mathrm{He}$ and $\mathrm{Ne}$ are excluded.

The fraction of complete vs. partial trapping is somewhat difficult to constrain given the quality of our current data and the uncertainties associated with the solubilities of the gases. We conducted a sensitivity study where we ran the model five times, each with approximately the same amount of total air injection but 
Table 4.9: The parameter values calculated using the nonlinear optimization scheme when one or more gases was excluded from the cost function. The base case is included in the first line for reference purposes. The air injection fluxes $F_{M G}$ in mol m${ }^{-2} \mathrm{~s}^{-1}$ of the sum of all the major gases $\left(\mathrm{N}_{2}, \mathrm{O}_{2}\right.$, and Ar) is listed for the complete trapping flux, the partial trapping flux, and total air injection flux for all the cases.

\begin{tabular}{ccccccc}
\hline Gas(es) Excluded & $\gamma_{G}$ & $A_{c}\left(\mathrm{x} 10^{3}\right)$ & $A_{p}\left(\mathrm{x} 10^{-5}\right)$ & Complete $_{M G}$ & Partial $\mathrm{F}_{M G}$ & Total $\mathrm{F}_{M G}$ \\
\hline none & 0.79 & 3.21 & 1.64 & $1.23 \times 10^{-6}$ & $1.39 \times 10^{-7}$ & $1.37 \times 10^{-6}$ \\
$\mathrm{He}$ & 0.78 & 2.56 & 2.75 & $9.80 \times 10^{-7}$ & $2.34 \times 10^{-7}$ & $1.21 \times 10^{-6}$ \\
$\mathrm{Ne}$ & 0.84 & 4.48 & 0 & $1.71 \times 10^{-6}$ & 0 & $1.71 \times 10^{-6}$ \\
$\mathrm{Ar}$ & 0.87 & 3.37 & 2.18 & $1.29 \times 10^{-6}$ & $1.86 \times 10^{-7}$ & $1.48 \times 10^{-6}$ \\
$\mathrm{Kr}$ & 0.76 & 3.11 & 1.53 & $1.19 \times 10^{-6}$ & $1.30 \times 10^{-7}$ & $1.32 \times 10^{-6}$ \\
$\mathrm{Xe}$ & 0.75 & 3.10 & 1.45 & $1.19 \times 10^{-6}$ & $1.23 \times 10^{-7}$ & $1.31 \times 10^{-6}$ \\
$\mathrm{He}$ and Ne & 0.84 & 4.20 & 0.41 & $1.61 \times 10^{-6}$ & $3.52 \times 10^{-8}$ & $1.64 \times 10^{-6}$ \\
$\mathrm{Kr}$ and Xe & 0.71 & 2.94 & 1.36 & $1.13 \times 10^{-6}$ & $1.16 \times 10^{-7}$ & $1.24 \times 10^{-6}$ \\
\hline
\end{tabular}

with a different percentage of complete trapping, ranging from $0 \%$ to $100 \%$ (Fig. 4-10). The ratio of He to Ne saturation anomalies changes with different fractions of complete trapping. However, our He and $\mathrm{Ne}$ data have uncertainties of roughly $1 \%$, preventing us from using $\mathrm{He}$ and $\mathrm{Ne}$ as a tight constraint on the fraction of complete vs. partial trapping. Interestingly, the winter saturation anomaly of $\mathrm{Ar}, \mathrm{Kr}$, and especially $\mathrm{Xe}$ increase as the fraction of partial trapping increases. Since partial trapping is proportional to the solubility of the noble gases and the heavier gases are more soluble, they are much more affected by partial than by complete trapping. At the moment, given the solublity uncertainty in Xe, we cannot use Xe to constrain the fraction of complete vs. partial trapping. However, if the solubility uncertainties in Xe were resolved, then Xe could offer a powerful tool for distinguishing between partial and complete trapping.

\subsubsection{Sensitivity of Parameters}

We examined the robustness of the choice of parameters by running the inverse scheme with various sets of physical parameters, different $\sigma_{\Delta}$, different proportions between the upper ocean saturation anomaly component and the deep concentration component of the cost function. We also explored including or 
Figure 4-10: Sensitivity of the surface saturation anomalies of the five noble gases to changes in the proportion of complete vs. partial trapping. All five runs have similar total amounts of air injection. The model run with the largest fraction of complete trapping (red) has the lowest winter surface saturation anomalies of the heavier noble gases. Xe is the most sensitive gas to the proportion of complete vs. partial trapping. Please note the difference in scale between the $\mathrm{He}$ and $\mathrm{Ne}$ plots and the $\mathrm{Ar}, \mathrm{Kr}$, and $\mathrm{Xe}$ plots.
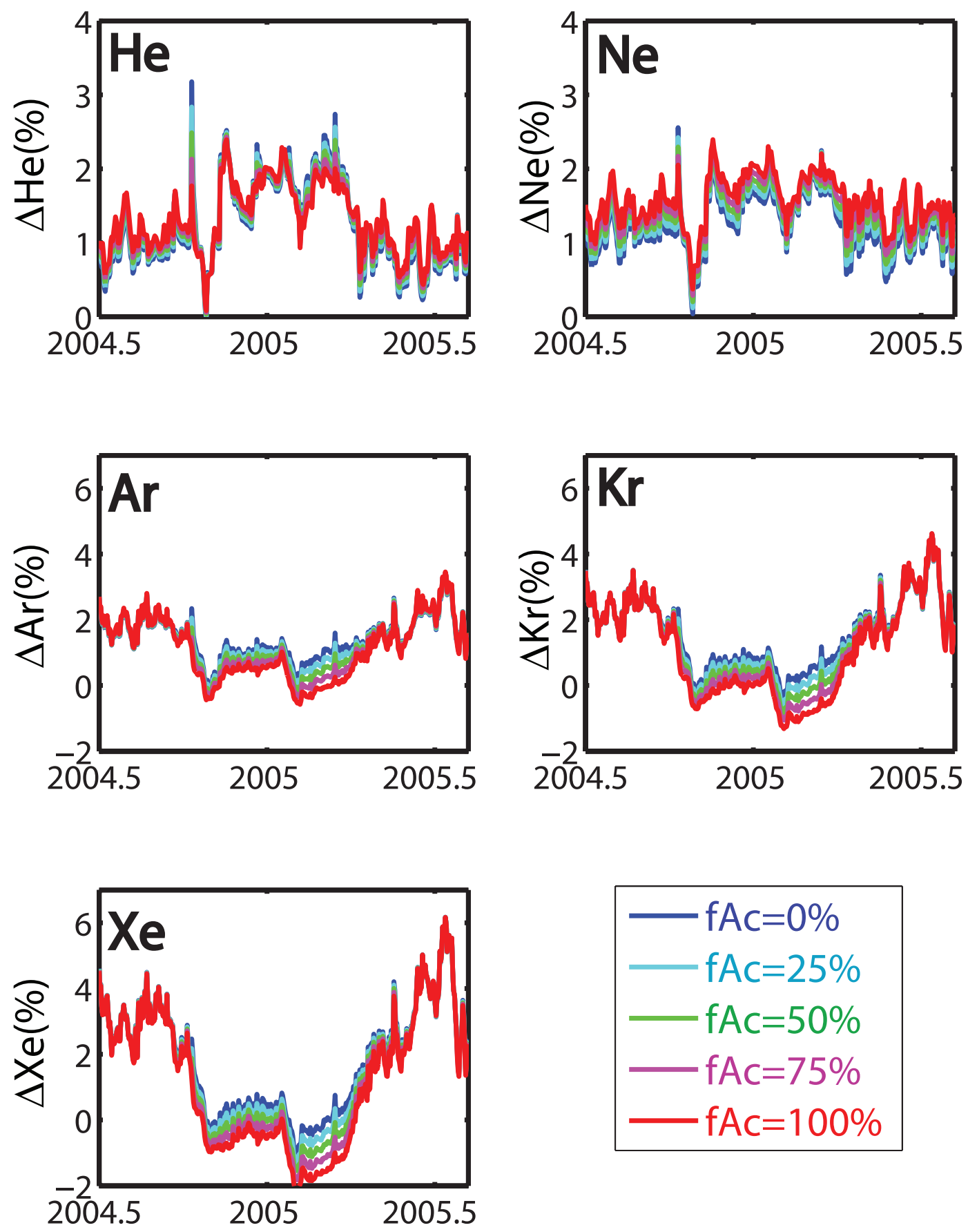
excluding the seasonal amplitude of saturation anomalies and including a constant data/model offset to potentially account for problems with our model.

The magnitude of diffusive gas exchange varies by $\pm 6 \%$ depending on the set of physical parameters used to run the model. The total air injection and the partitioning between complete and partial traping change by approximately $6 \%$ depending on the set of physical parameters used. Since we cannot distinguish between the three sets of physical parameters, this $\pm 6 \%$ range is a source of uncertainty in our air-sea gas exchange parameters.

The gas exchange parameters are sensitive to the choice of $\sigma_{\Delta}$. We include in $\sigma_{\Delta}$ (1) the uncertainty in sampling and analysis, assessed by the deviation between duplicate pairs, (2) the solubility uncertainties in the noble gases and (3) the standard error in our estimates of average mixed layer saturation anomaly (see Table 4.4). The first and third of these contributions are clearly defined. The second contribution, i.e. the uncertainties in the solubility values, is not well known. The uncertainties in the solubility determination of $\mathrm{Ne}$ and $\mathrm{Ar}$ are stated by Hamme and Emerson (2004b) to be $0.3 \%$ and $0.13 \%$ respectively. For the other gases, however, the estimates are less clear. For He, we apply a correction ranging from $0.5 \%$ to $1.5 \%$ to the Weiss (1971) solubility values. We estimate the uncertainty in the corrected He solubility value to be $0.5 \%$ since the estimated uncertainty in uncorrected solubility values is $1 \%$ and the correction should improve the values by at least a factor of 2 . For Kr, Weiss and Kyser (1978) list the uncertainty as $0.4 \%$. We have used an uncertainty of $1 \%$ in most of our calculations since we expect the Weiss and Kyser $\mathrm{Kr}$ values to be no better than the $\mathrm{He}$ or $\mathrm{Ne}$ values. The solubility of Xe is the most uncertain, but exactly how uncertain is not clear. Hamme and Severinghaus (2007) suggest the Xe solubility values may be in error by $2 \%$ based on deviation of an ocean Xe profile from expected values. However, that study does not offer a firm constraint on the uncertainty in Xe solubility since the estimate is simply based on their data not meeting their expectation.

In order to better assess the uncertainty in Xe solubility, and indeed in the solubility of all the gases, we compared the freshwater versions of the "standard" set of solubility relationships used in this study with freshwater solubility values derived by Krause and Benson (1989) (Fig. 4-11). By "standard", we are referring to the solubility relationships used here, e.g. modified Weiss (1971) for He, Hamme and Emerson (2004b) for Ne and Ar, Weiss and Kyser (1978) for Kr and Wood and Caputi (1966) for Xe. The Krause and Benson (1989) measurements (referred to here as KB) were carefully made using pressure measurements 
and have uncertainties of less than 0.1\%. However, the Krause and Benson (1989) measurements were only made in freshwater and thus we cannot use them directly for our study. Nonetheless a comparison between them and the freshwater versions of the standard solubility relations gives us insight on the possible uncertainties in the standard solubility relationships. The deviations of $\mathrm{Ne}$, and $\mathrm{Ar}$ from the $\mathrm{KB}$ values are less than $0.3 \%$, and are on average $0.04 \%$ for $\mathrm{Ne}$ and $0.14 \%$ for Ar. Our "Hamme-corrected" He solubility relationship differs from the $\mathrm{KB}$ values by at most $0.8 \%$ at $18{ }^{\circ} \mathrm{C}$ and on average by $0.3 \%$, suggesting that an error estimate of $0.5 \%$ for $\mathrm{He}$ is a reasonable choice. The standard $\mathrm{Kr}$ solubility values differ from $\mathrm{KB}$ by at most $1.1 \%$, and on average by $0.8 \%$, suggesting our $1 \%$ estimate was reasonable, but perhaps too pessimistic. We conducted an inverse run using a $0.5 \%$ estimated uncertainty in $\mathrm{Kr}$ values. The standard Xe solubility value differs from the $\mathrm{KB}$ values by at most $1.5 \%$ but because the value is $1.5 \%$ higher than the $\mathrm{KB}$ value at low temperatures, and $1.5 \%$ lower than the $\mathrm{KB}$ values at high temperature, the average deviation is only $0.11 \%$. We thus investigated the sensitivity of our results to a choice of $1 \%, 1.5 \%$ and $2 \%$ estimated uncertainty in Xe solubility.

The values of the diffusive gas exchange parameter changed by at most $\pm 4 \%$, the total air injection flux changed by at most $\pm 4 \%$, and the partitioning between partial and complete trapping changed by at most $\pm 2 \%$ depending on the choice of uncertainty in the solubility of $\mathrm{Kr}$ or $\mathrm{Xe}$ (within the range described above). If, however, the uncertainty in solubility values were not included for any of the gases (in other words, if assumed that we knew all the solubilities perfectly), then the results changed substantially for air injection but only by $10 \%$ for diffusive gas exchange: $\gamma_{G}=0.87, A_{C}=2.98 \times 10^{3}$, and $A_{P}=3.6 \times 10^{5}$. Using the parameters from this extreme case is clearly not justified since we know there are substantial uncertainties in the solubilities of the noble gases. Nonetheless, the fact that there is no big difference for diffusive gas exchange is reassuring. The large difference for air injection suggests the need for accurate determination of $\mathrm{Kr}$ and $\mathrm{Xe}$ solubilities.

We also performed one run where we weighted the gas contributions in the cost functions by the variance of the observations. The advantage of this is then each of the gases has a similar contribution to the cost function - otherwise since Xe has the widest range in surface saturation anomalies, changes in Xe have a disproportionate effect on the cost function. The magnitude of diffusive gas exchange did not change and the magnitude of the air injection flux changed by only $1 \%$ if the variances were included. The proportion 
Figure 4-11: The ratios of solubility concentrations used in this study (denoted as $\alpha_{X}$ ) to the Krause and Benson solubility concentrations (denoted $\alpha_{K B}$ ) for freshwater from $18{ }^{\circ} \mathrm{C}$ to $30{ }^{\circ} \mathrm{C}$. The deviation of this ratio from 1 gives a measure of the size of the uncertainty in the solubility values used. However, it does not provide a valid way of "correcting" the solubility values used since Krause and Benson only determined solubility for freshwater and our study is for seawater. Neon and Ar solubility values agree the most closely whereas Xe solubility values are the most different.
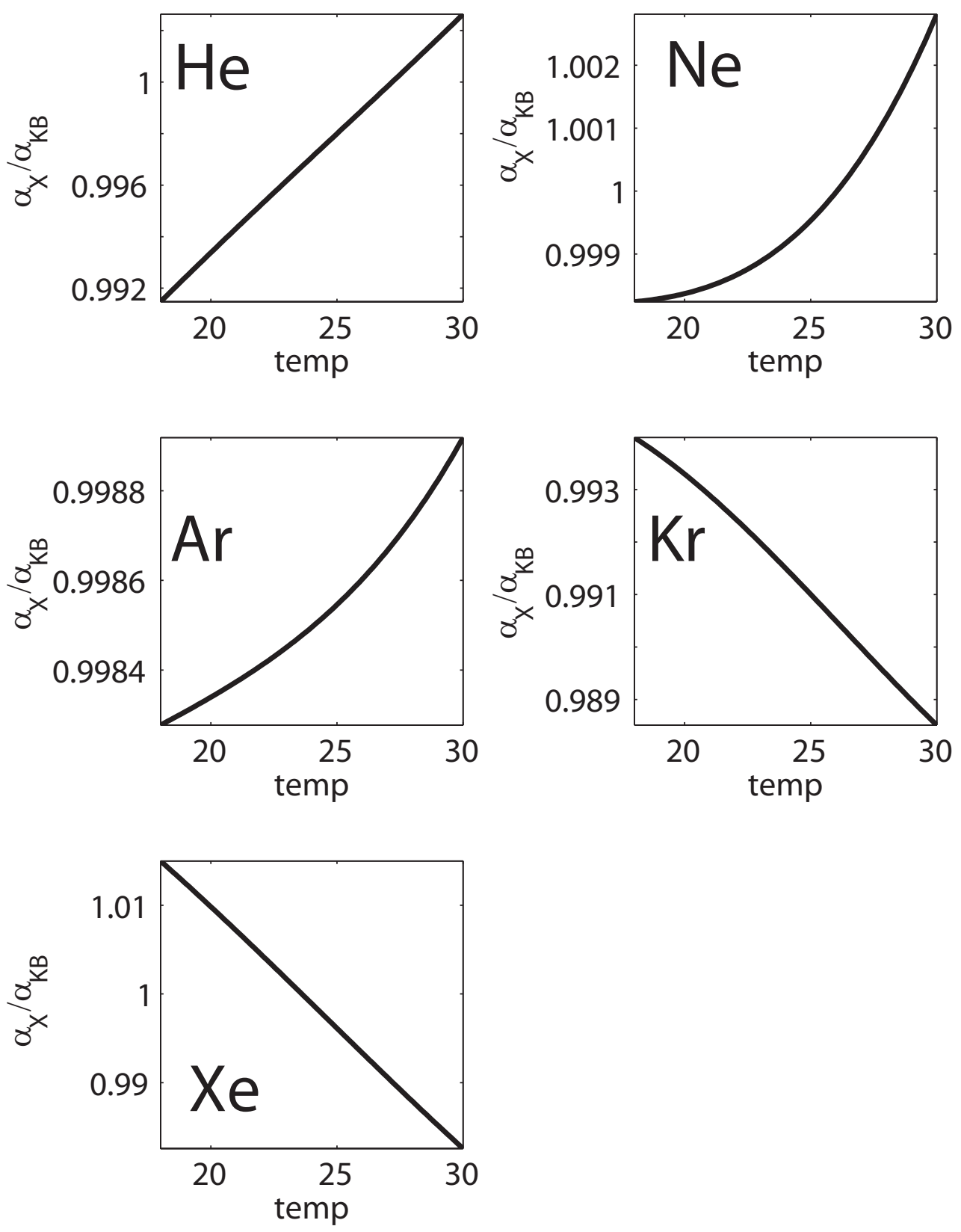
of complete vs. partial trapping was more sensitive and changed by approximately $4 \%$.

Our cost function has two components - a mixed layer saturation anomaly component and a deep concentration component. In the base case, these two components are approximately equal. We explored the sensitivity of the parameters to relative contributions of these components by changing $a_{1}$ and $a_{2}$ so that the surface component was either twice as large or half the size as the deep component. The diffusive gas exchange fluxes and the air injection fluxes change by less than $3 \%$ suggesting the parameters are not sensitive to the balance between the two components.

Our base case uses the actual values of the saturation anomalies in the cost function. A different possible formulation of the cost function includes the seasonal amplitude of the saturation anomalies of the noble gases. The advantage of this approach is that it would mitigate any systematic offsets in our model or in the solubility values. We thus performed runs where we added in a component of the cost function to reflect the deviation in seasonal amplitude of the surface saturation anomalies between the data and the model. We either weighted both the seasonal amplitude and the absolute magnitude of the surface saturation anomalies equally (run \#10) or weighted the seasonal amplitude as only $25 \%$ of the mixed layer component of the cost function and the absolute magnitude as $75 \%$ (run \#11). The values of $\gamma_{G}$ changed by at most $4 \%$, the total air injection flux by $15 \%$, and the partitioning between partial and complete trapping only change by $10 \%$ if seasonal amplitudes are included in the cost function. When the seasonal amplitude was included, the total air injection flux decreased and the fraction due to partial trapping increased.

The biggest changes in our parameters occur if we include a constant offset in the data/model comparison. For example, the model does not directly treat lateral advection which could lead to uncertainties in especially $\mathrm{Kr}$ and $\mathrm{Xe}$. We examined what effect a problem in the model would cause on our choice of parameters by adding a constant offset to our model-data comparison. This offset differed for each gas; since $\mathrm{Xe}$ is more sensitive to thermal changes, it may be more sensitive to problems with the model. We tried two choices of constant offsets: $0.5 \%, 0.75 \%$, and $1 \%$ for $\Delta \mathrm{Ar}, \Delta \mathrm{Kr}$, and $\Delta \mathrm{Xe}$ respectively in run \#12, and $0.25 \%, 0.375 \%$, and $0.5 \%$ respectively for $\Delta \mathrm{Ar}, \Delta \mathrm{Kr}$ and $\Delta \mathrm{Xe}$ in run $\# 13$. We chose the relative sizes of these offsets by comparing the change in solubility value with temperature for the gases. In particular, arguments by Ito and Deutsch (2006) show that the heavier gases are supersaturated in the subsurface as a result of diapcynal mixing. We calculated that if Ar was $0.5 \%$ supersaturated due to diapycnal mixing, then 
$\mathrm{Kr}$ would be $0.75 \%$ and Xe would be $1 \%$ supersaturated. Although we should be accounting for diapycnal mixing in our model through our choice of the vertical diffusivity constant, $K_{z}$, these numbers seemed a reasonable starting point for considering data/model offsets.

The diffusive gas exchange parameter, $\gamma_{G}$ increased by $30 \%$ if we included a constant data-model offset of $0.5 \%, 0.75 \%$, and $1 \%$ for $\Delta \mathrm{Ar}, \Delta \mathrm{Kr}$, and $\Delta \mathrm{Xe}$ respectively. The total air injection flux increased by $6 \%$ for He and decreased by $20 \%$ for Xe. Moreover, the proportion of air injection switched to entirely complete trapping; since the Xe winter saturation anomalies are now "matched" by the model (given a $+1 \%$ model offset), the air injection must be completely trapped in order not to add any more Xe in the winter-time. These large changes suggest that the results are sensitive to model dynamics and thus any errors in model dynamics will propagate into determination of gas exchange parameters. Further work on improving this model or on a more complicated model that includes lateral advection might be worthwhile. Additionally, a longer time-series will help resolve whether the model-data discrepencies occur every year. We have samples from three full years but we have only analyzed one year of samples for this study.

\subsection{Conclusions}

In this study, we use a 14 month time-series of five noble gases to determine parameters for calculating the fluxes of air-sea gas exchange, explicitly separating and quantifying diffusive gas exchange, complete bubble trapping, and partial bubble trapping. The parameters for both air injection and diffusive gas exchange determined here and their associated equations can now be applied to calculate the air-sea flux of any gas of interest. The magnitude of diffusive gas exchange is approximately $20 \%$ lower than predicted by Wanninkhof (1992) and in the same range as more recent estimates by Ho et al. (2006) and Sweeney et al. (2007). Indeed, the magnitude of diffusive gas exchange in the parameterization presented here agrees within $2 \%$ to that of Ho et al. (2006) and is $11 \%$ lower than that of (Sweeney et al., 2007). However, such agreement is deceptive since Ho et al. and Sweeney et al. did not explicitly include air injection in their parameterizations. In tracer release experiments, ${ }^{3} \mathrm{He}$ and $\mathrm{SF}_{6}$ are added to the water, driving a diffusive gas exchange flux out of the mixed layer. Partial bubble trapping should also result in a flux out of the water, since the ${ }^{3} \mathrm{He}$ and $\mathrm{SF}_{6}$ could diffuse into the bubbles and then escape to the atmosphere. Thus the tracer 
release experiment gives the flux due to diffusive gas exchange and partial trapping but does not account for complete trapping. In the case of radiocarbon (Sweeney et al., 2007), the influx of ${ }^{14} \mathrm{C}$ into the water is considered and therefore in these studies diffusive gas exchange and air injection (both partial and complete) are working together. In contrast, the parameterization presented here separately considers each type of flux and therefore contains three separate parts for calculating the flux of diffusive gas exchange, partially trapped bubbles, and completely trapped bubbles.

Relatively little previous work has been done on estimating sizes of air injection fluxes. Additionally, the air injection flux can change dramatically with the wind speed (given the cubic dependence) and thus comparing estimates of air injection at different locations and times is difficult. The $\mathrm{O}_{2}$ bubble flux estimated by Hamme and Emerson (2006) at station ALOHA in Hawaii is $7 \times 10^{-8} \mathrm{~mol} \mathrm{~m}^{-2} \mathrm{~s}^{-1}$, which is more than three orders of magnitude smaller than the $\mathrm{O}_{2}$ bubble flux of $1 \times 10^{-4} \mathrm{~mol} \mathrm{~m}^{-2} \mathrm{~s}^{-1}$ estimated by Zhang et al. (2006) during a winter storm at station PAPA in the Northeast Pacific. Our estimates fit within this wide range. Our "summer" or low wind speed estimate of air injection flux for $\mathrm{O}_{2}$ (calculated at $\mathrm{u}_{10}=7 \mathrm{~m} \mathrm{~s}^{-1}$ ) is approximately $8.6 \times 10^{-8} \mathrm{~mol} \mathrm{~m}^{-2} \mathrm{~s}^{-1}$, comparable to Hamme and Emerson's value. Our "winter" or high wind speed air injection flux for $\mathrm{O}_{2}$ (calculated at $\mathrm{u}_{10}=15 \mathrm{~m} \mathrm{~s}^{-1}$ ) is $2.0 \times 10^{-6} \mathrm{~mol} \mathrm{~m}^{-2} \mathrm{~s}^{-1}$. In very high wind speeds $\left(\mathrm{u}_{10}=25 \mathrm{~m} \mathrm{~s}^{-1}\right)$ comparable to the winter storm wind speed seen by Zhang et al. (2006), we estimate an $\mathrm{O}_{2}$ bubble flux of $1.4 \times 10^{-5} \mathrm{~mol} \mathrm{~m}^{-2} \mathrm{~s}^{-1}$. This is still an order of magnitude below the estimate of Zhang et al. The extrapolation of our results to wind speeds of $25 \mathrm{~m} \mathrm{~s}^{-1}$ may not be justified since our time series data was collected with winds primarily less than $15 \mathrm{~m} \mathrm{~s}^{-1}$ and thus any extrapolation is sensitive to the assumption of a cubic dependence on wind speed.

The partitioning between complete and partial trapping we calculate here is within the range of that predicted by Hamme and Emerson (2006). We found that in the winter, completely and partially trapped bubbles lead to significant contributions in the saturation anomalies of even soluble gases, suggesting that air injection may be more important than previously thought for soluble gases, such as $\mathrm{CO}_{2}$.

We examined the sensitivity of our parameters to a range of conditions and found the results to be consistent to within approximately $\pm 6 \%$ for the magnitude of diffusive gas exchange, and $\pm 15 \%$ for the air injection fluxes. The root mean square wind speed over the time-series was $6.7 \mathrm{~m} \mathrm{~s}^{-1} \pm 6.6 \mathrm{~m} \mathrm{~s}^{-1}$. This suggests that our parameters are best known at $\sim 7 \mathrm{~m} \mathrm{~s}^{-1}$ and the parameterization is valid between 0 and 
$13 \mathrm{~m} \mathrm{~s}^{-1}$ (within one standard deviation of the mean). Additionally, the parameterization is only valid for similarly oligotrophic waters. Coastal waters have surfactants that can depress the gas exchange (Frew et al., 2004).

Additionally, as a cautionary note, however, the consistency of these values does not necessarily ensure their accuracy. Our biggest sources of uncertainty are the possibility of systematic problems with our model and with the solubility functions of the heavier noble gases. It thus would be worthwhile to revisit the same data-set with a more complete model. We tried to account for the uncertainty in the solubilities by the weights in our cost function, but nonetheless, the need for more accurate solubility determinations of the heavier noble gases is critical.

Once we have an improved model and more accurate solubility values, we could examine the effect of different wind products on our parameter choice. The Bermuda Testbed Mooring (Dickey et al., 1998) provides a record of wind speeds measured at a mooring nearby our sample collection. The record has gaps during times when the mooring was not deployed but nonetheless it offers the valuable chance to link direct wind measurements with gas exchange processes. Additionally, we should examine the effect of using the NCEP reanalysis wind product on our parameters. Since NCEP and QuikSCAT have different mean $u_{10}^{2}$, we would have calculated different parameterizations if we used a different wind product. Thus the parameters calculated here should either be applied only to QuikSCAT winds or the appropriate corrections should be made. Since many researchers use NCEP winds, it would be useful to determine a set of parameters that is consistent with NCEP winds.

Additionally, it would be interesting to use our time-series of noble gas data collected here in combination with satellite altimetry and backscatter measurements in order to calibrate air-sea gas exchange flux parameterized as a function of surface roughness as well as wind speed (Woolf, 2005; Glover et al., 2007; Frew et al., submitted, 2007). Some of the variability in existing parameterizations of air-sea gas exchange may be because the diffusive part of the gas transfer rate does not directly depend on wind speed but rather on the frequency spectrum of the divergence field at the surface and thus is a function of surface roughness, surfactant films, sea state, etc. The data set collected here, in combination with satellite records of surface altimetry and mean square slope, could offer the valuable opportunity for constraining a parameterization of air-sea gas exchange based on surface roughness. 


\subsection{Acknowledgments}

This work was supported by the National Science Foundation Chemical Oceanography program (OCE0221247) and by the Department of Defense (graduate fellowship to RHRS). We would like to thank the captain and crew of the $R / V$ Weatherbird II, the $R / V$ Cape Hatteras, and the $R / V$ Oceanus for their assistance in collecting samples. We are grateful to Mike Lomas, Rod Johnson, Megan Roadman, and all other BATS personnel for the use of lab-space, ship-time, frequent help and good cheer. We thank Carolyn Walker, Dempsey Lott, and Kevin Cahill for collecting samples on three of the cruises. We would also like to thank DeDe Toole and Naomi Levine for their generous donation of computing time. 
Chapter 5

Estimates of Biological Production from a Time-Series of Noble Gases, Tritium, and Helium-3 


\begin{abstract}
A fourteen month time-series of ${ }^{3} \mathrm{He}$, tritium, oxygen, and noble gases collected in the Sargasso Sea between July 2004 and August 2005 is used to quantify new production, export production, and net community production. The flux of ${ }^{3} \mathrm{He}$ from the mixed layer is used to estimate new production of $0.9 \pm 0.3 \mathrm{~mol} \mathrm{~N}$ $\mathrm{m}^{-2} \mathrm{y}^{-1}$, which is equivalent to $10 \pm 3 \mathrm{~mol} \mathrm{O}_{2} \mathrm{~m}^{-2} \mathrm{y}^{-1}$. Apparent oxygen utilization rates calculated from tritium/helium dating of thermocline waters lead to estimates of export production of 3.7 to $5.0 \mathrm{~mol} \mathrm{O}_{2} \mathrm{~m}^{-2}$ $\mathrm{y}^{-1}$. Euphotic zone seasonal cycles of $\mathrm{O}_{2}$ and Ar, combined with an upper ocean one-dimensional vertical model, result in estimates of net community production of 3.0 to $5.2 \mathrm{~mol} \mathrm{O}_{2} \mathrm{~m}^{-2} \mathrm{y}^{-1}$. The discrepancy between the new production estimate vs. the estimates of export and net community production may reflect a larger than expected contribution of biological production in the winter or may be a result of uncertainties and differences in the temporal and spatial scales inherent in the methods. The ${ }^{3} \mathrm{He}$ flux gauge and apparent oxygen utilization rates yield a regional scale estimate of new production whereas the euphotic zone $\mathrm{O}_{2}$ and Ar cycles give a local, immediate estimate of net community production.
\end{abstract}




\subsection{Introduction}

A wide range of methods that average over various space and time scales have been applied to determine the amount of carbon exported from the euphotic zone. Upper ocean sediment traps offer the advantage of direct collection of sinking particles but likely have collection biases due to swimmers and hydrodynamic flow across the mouth of the traps (Gardner, 2000). Neutrally buoyant sediment traps likely resolve the hydrodynamic issue (Buesseler et al., 2000; Valdes and Price, 2000; Stanley et al., 2004). However, sediment traps average over time scales of a few days and thus may easily miss or be biased by large episodic

sinking events. Export can be estimated from ${ }^{234} \mathrm{Th}$ disequilibria and Th:C ratios, with such estimates differing from sediment traps by as much as a factor of three to ten (Buesseler, 1991; Buesseler et al., 1994; Murray et al., 1996; Hernes et al., 2001). Radiotracer incubation bottle experiments offer a snapshot of the new production at a particular time and place (Dugdale and Goering, 1967) but again spatial and temporal heterogeneity can make such snapshots difficult to interpret. Additionally, so-called bottle effects cause by confining production to a bottle and thus subsampling the community, disrupting grazing, potential leaching of chemicals from the bottle, etc. can lead to difficulty in interpreting the bottle estimates of production. Episodic events such as eddies (McGillicuddy et al., 1998, 1999; Sweeney et al., 2003; Mourino-Carballido and McGillicuddy, 2006; McGillicuddy et al., 2007) may constitute a large fraction of new production and might be missed by bottle experiments or sediment traps.

Satellite measurements of ocean color, and by extension of net primary production (NPP), are extremely useful because of detailed spatial and temporal coverage (Behrenfeld et al., 2005). However, satellites measure only water characteristics down to one optical depth and they measure ocean color, not NPP directly; estimates of NPP from ocean color via models have errors on the order of a factor of two (Carr et al., 2006). Satellites may not accurately track production at the base of the euphotic zone. Moreover, satellites give a measure of the inventory of chlorophyll. The relationship between the standing stock of chlorophyll and productivity is not necessarily constant. Additionally, net community production (NCP), rather than NPP, is the most climatically relevant parameter (where NCP is defined as NPP minus heterotrophic respiration), and NCP is difficult to estimate from NPP.

Geochemical tracers are ideal tools for studying rates of biological production because they allow for collection of large amounts of data and because they characterize the integrated behavior of systems over 
broad spatial and temporal scales. Tracers thus complement methods such as satellites, which offer detailed coverage but are hard to relate to NCP, and they complement sediment traps and incubation experiments, which provide a snapshot of one particular location at one particular time. A variety of geochemical tracers have been used near Bermuda in the oligotrophic Sargasso Sea to quantify three types of production - net community production, export production, and new production. Export production is defined as the flux of organic matter leaving the euphotic zone. New production is defined as the flux of new nutrients into the euphotic zone, primarily through upwelling or upward mixing of thermocline waters (Dugdale and Goering, 1967). Over sufficiently long temporal and spatial scales, the three types of production should be in balance (Eppley and Peterson, 1979). In this study we use a fourteen month time-series of ${ }^{3} \mathrm{He}$, tritium, $\mathrm{O}_{2}$, and $\mathrm{Ar}$ measured at the Bermuda Atlantic Time-series Study (BATS) site between July 2004 and August 2005 in order to measure new production through the use of the ${ }^{3} \mathrm{He}$ flux gauge technique (Jenkins, 1988b; Jenkins and Doney, 2003), export production from apparent oxygen utilization rates (Riley, 1951; Jenkins, 1977, 1980), and NCP from euphotic zone seasonal cycles of $\mathrm{O}_{2}$ and Ar (Craig and Hayward, 1987; Emerson, 1987; Spitzer and Jenkins, 1989).

The principle behind the ${ }^{3} \mathrm{He}$ flux gauge technique is that nitrate and excess ${ }^{3} \mathrm{He}$ are correlated in the thermocline because as water ages, it gains in nitrate due to continuing remineralization and gains in ${ }^{3} \mathrm{He}$ due to decay of tritium. When this water is mixed into the euphotic zone, it supplies the the nutrients needed for new production and also carries an excess ${ }^{3} \mathrm{He}$ signal. We can measure excess ${ }^{3} \mathrm{He}$ in the mixed layer and use a gas exchange relationship to calculate the air-sea flux of excess ${ }^{3} \mathrm{He}$ to the atmosphere. This excess ${ }^{3} \mathrm{He}$ flux out of the mixed layer must be balanced by a supply of excess ${ }^{3} \mathrm{He}$ from below on annual or longer time scales, and thus gives us a measure of the amount of excess ${ }^{3} \mathrm{He}$ being input into the mixed layer. We then use the correlation between excess ${ }^{3} \mathrm{He}$ and nitrate in order to predict the input of nitrate. Therefore, this calculation predicts new production based on the physically mediated nitrate flux from vertical transport of thermocline waters - it does not take into account nitrate from other sources such as nitrogen fixation or zooplankton migration (Steinberg et al., 2000). Excess ${ }^{3} \mathrm{He}$ must be transported physically and we assume that the physical transport (i.e. advection or turbulent mixing) does not discriminate between excess ${ }^{3} \mathrm{He}$, nitrate, phosphate, or any other constituent of the water. Additionally, it assumes that the water is coming from the thermocline and thus the correlation observed between nitrate and excess ${ }^{3} \mathrm{He}$ for the thermocline 
can be used.

${ }^{3} \mathrm{He}$ and tritium can be used in the aphotic zone to calculate apparent oxygen utilization rates (AOUR). ${ }^{3} \mathrm{He}$ and tritium are used as a "clock" in order to estimate the ventilation age of the water (Jenkins and Clarke, 1976). When the water is at the surface, excess ${ }^{3} \mathrm{He}$ is almost completely lost due to gas exchange. As water is sequestered from the atmosphere and ages, ${ }^{3} \mathrm{He}$ builds up and tritium decreases. In practice, mixing complicates matters and thus we use a simple box model in order to estimate ventilation time scales (Jenkins, 1980). The apparent oxygen utilization (AOU) - the difference between equilibrium $\mathrm{O}_{2}$ and measured $\mathrm{O}_{2}-$ results from $\mathrm{O}_{2}$ being consumed during remineralization of exported matter. By combining the ventilation age of the water in the aphotic zone with the AOU, we can calculate the apparent oxygen utilization rate (AOUR). The vertically integrated AOUR is a measure of export production.

In the euphotic zone, $\mathrm{O}_{2}$ can be used to estimate NCP. In the summer, $\mathrm{O}_{2}$ produced by photosynthesis is trapped between the bottom of the mixed layer and the bottom of the euphotic zone, resulting in a subsurface oxygen maximum. Argon can be used as an abiotic analogue of $\mathrm{O}_{2}$ to distinguish the physical component of this supersaturation from the biological one, and thus to quantify the net community production. Oxygen and Ar, however, have different boundary conditions below the euphotic zone, as there is a sharp gradient in $\mathrm{O}_{2}$ concentration due to remineralization. Thus we use a one-dimensional, vertical, modified Price-WellerPinkel model (Price et al., 1986; Spitzer and Jenkins, 1989; Stanley et al., 2006) and five noble gases in order to characterize the physical component of this supersaturation. We then inversely use the model to determine the biological activity required to produce the observed $\mathrm{O}_{2}$ signals.

This study is unique in that it measures all three types of production - NCP, new production, and export production - using geochemical tracers at the same time in the same place. We begin to make inferences from comparing the types of production, given of course the caveats in our methodology. In section 5.2 of this paper, we describe in detail each method used. In section 5.3, we present the results for each type of production estimated and discuss the limitations of each method. In section 5.4, we synthesize the results and reflect how they relate to the broad questions of nutrient cycling and biological production. 


\subsection{Methods}

\subsubsection{Data Collection}

Helium Isotopes and Noble Gases Helium isotopes were measured on the same samples that were used for noble gas analyses. Please see Chapter 4 for complete details on sample collection, including exact location and time of samples. In brief, samples were collected aboard the $R / V$ Weatherbird II, the $R / V$ Cape Hatteras, or the $R / V$ Oceanus at the Bermuda Atlantic Time-series Study (BATS) site every month between July 2004 and August 2005 at 22 depths in the upper $400 \mathrm{~m}$ of the water column. Additionally, samples were collected down to $4200 \mathrm{~m}$ during November 2004 and April 2005. On all cruises except for the February 2005 cruise, the samples were collected on a "core" BATS cruise. The samples were collected in valved stainless steel sample cylinders ( $90 \mathrm{cc}$ volume) by gravity feeding through tygon tubing from Niskin bottles. The gases were extracted from the samples into aluminosilicate glass bulbs (approximate volume of 25 cc) in the on-shore laboratory at the Bermuda Biological Station. Details of the sampling and extraction procedures are described in Lott and Jenkins (1998).

Samples were extracted as soon as possible - usually within 24 hours of sample collection - because He permeates through the viton o-rings in the cylinder plug valves. Experiments conducted with degassed water suggest that samples are compromised at a rate of $0.46 \%$ of their He disequilbrium per day. Thus for a sample with a $2 \%$ difference in He isotope ratio, a 24 hour delay would lead to a signal reduction of $0.009 \%$. The aluminosilicate bulbs were brought to Woods Hole Oceanographic Institution and stored in ambient conditions for up to two years before analysis. Because the gases have a practically zero permeation rate through aluminosilicate glass, the only effect of this storage time on our measurements is for the decay of tritium in water in the sample bulb to slightly elevate the He isotope ratio by approximately $0.02 \%$. This correction is small because when the samples were first collected, we extracted all the ${ }^{3} \mathrm{He}$ from the samples but only about $5 \%$ of the water and thus only about $5 \%$ of the tritium. This correction is small compared to the measurement uncertainty and is accounted for in our calculations.

The aluminosilicate bulbs are attached to a dual mass spectrometric system and analyzed for He isotopes as well as for $\mathrm{Ne}, \mathrm{Ar}, \mathrm{Kr}$, and Xe. For details of analytical procedures, please see Chapter 2 of this thesis. In short, the noble gases are purified and separated by being sequentially drawn through a two-stage water 
vapor cryotrap, a Pd catalyst, and Ti-Zr-Fe getters onto two cryogenic traps. Neon, Ar, Kr, and Xe sorb onto a stainless steel cryogenic trap and He sorbs onto an activated charcoal cryotrap. The charcoal cryotrap is warmed to $40 \mathrm{~K}$ and $\mathrm{He}$ is selectively released into the helium isotope mass spectrometer (HIMS). The HIMS, an improved system based on the "Clarke design" (Clarke et al., 1976), is a purposely constructed branch tube, statically operated, dual collector magnetic sector helium isotope mass spectrometer, radius of $25.4 \mathrm{~cm}$, equipped with a Faraday cup and a pulse counting SEM. The other noble gases were released sequentially from the stainless steel cryogenic trap into a statically operated quadrupole mass spectrometer (QMS) for measurement by peak height manometry. The QMS is a Hiden quadrupole mass spectrometer (P/N PCI 1000 1.2HAL/3F 1301-9 PIC type 570309) run in static mode, equipped with a pulse counting secondary electron multiplier (SEM). The system, including the processing line, cryotraps, and mass spectrometers, is operated under computer program control to achieve a high degree of reproducibility and for continuous operation.

Standards, consisting of precisely known aliquots of marine air, are used for reference for the He isotopes and for calculation of absolute amount of noble gases in the samples. These standards are processed in the same way as samples. Additionally, the measurements are corrected for non-linearities in the processing line and mass spectrometer. The linearity correction for He isotopes is typically around $0.01 \%$, which is smaller than our measurement uncertainty.

The helium isotope data are presented here as isotope ratio anomalies $\delta^{3} \mathrm{He}$ relative to the atmospheric standard and are defined as

$$
\delta^{3} \mathrm{He}=\left(\frac{R_{\text {smpl }}}{R_{\text {std }}}-1\right) \times 100
$$

where $R_{s m p l}=\left(\frac{{ }^{3} \mathrm{He}}{{ }^{4} \mathrm{He}}\right)_{s m p l}$ is the isotopic ratio in the sample and $R_{s t d}=\left({ }^{3} \mathrm{He}\right)_{s t d}$ is the isotopic ratio in an air standard. Measurement error in the isotope ratio anomaly as determined by reproducibility of air standards is $0.1 \%$ and as determined by reproducbility of sample replicate pairs is $0.14 \%$.

The argon and oxygen data are presented here as saturation anomalies $\Delta$, which are the percent deviation from solubility equilibrium, and defined as

$$
\Delta=\left(\frac{C_{i, w}}{C_{i, e q}}-1\right) \times 100
$$


where $C_{i, w}$ is the concentration of gas $i$ in the water and $C_{i, e q}$ is the concentration of gas $i$ at equilibrium (i.e. the solubility value). Positive (negative) saturation anomalies reflect that the gas is supersaturated (undersaturated), and the magnitude of the saturation anomalies corresponds to the magnitude of the departure from equilibrium. For Ar, we use the solubility value of Hamme and Emerson (2004b) and for $\mathrm{O}_{2}$ we use the solubility value of Weiss (1970).

Oxygen Oxygen concentrations were measured by BATS personnel on samples collected from the same Niskins as the noble gases. Oxygen concentration was determined by automated titration, according to the Carpenter (1965) modification of the traditional Winkler titration. Please see the BATS method manual (Knap et al., 1997) for more details. The precision of duplicates is typically better than $0.5 \mu \mathrm{mol} \mathrm{kg}^{-1}$.

Tritium Tritium samples were collected from Niskin bottles by gravity feeding through tygon tubing into $0.5 \mathrm{~L}$ or $0.95 \mathrm{~L}$ Boston round glass bottles that had previously been filled with Ar. The bottles were filled with seawater to the "shoulder", leaving about $100 \mathrm{cc}$ of Ar "blanket" present in order to minimize exchange with atmospheric water vapor. Samples were collected at the surface, $50 \mathrm{~m}, 100 \mathrm{~m}, 140 \mathrm{~m}, 200 \mathrm{~m}, 250 \mathrm{~m}$, 300 m, and 400 m every month. Additionally in Nov 2004 and April 2005, samples were collected at an additional 22 depths between $500 \mathrm{~m}$ and $4200 \mathrm{~m}$.

The bottles were brought to Woods Hole Oceanographic Institution and within typically two weeks were "degassed" into aluminosilicate glass bulbs (volume of $200 \mathrm{cc}$ or $500 \mathrm{cc}$ ). For degassing, the empty bulbs were connected to a high vacuum manifold, the seawater sample was transferred using negative pressure under an Ar "blanket" from the glass bottle to half-fill each bulb, and the bulb was repeatedly shaken and pumped in order to completely degas the water. After six cycles of shaking and pumping, the bulbs were flame-sealed and stored in the basement to shield from cosmogenically produced ${ }^{3} \mathrm{He}$. Samples from depths shallower than or equal to $400 \mathrm{~m}$ were collected in $0.5 \mathrm{~L}$ bottles and degassed in $200 \mathrm{cc}$ bulbs. Samples from deeper than $400 \mathrm{~m}$ were collected in $0.95 \mathrm{~L}$ bottles and degassed into $500 \mathrm{cc}$ bulbs. For more details on the degassing procedure, see Lott and Jenkins (1998).

After allowing at least six months for ingrowth of ${ }^{3} \mathrm{He}$ from tritium decay, the samples were analyzed for ${ }^{3} \mathrm{He}$ on a mass spectrometer, which though different than the one that measured the noble gas and helium isotope samples, is constructed of a similar design. It is also a purposely constructed branch tube, statically 
operated, dual collector magnetic sector helium isotope mass spectrometer, radius of $25.4 \mathrm{~cm}$, equipped with a Faraday cup and a pulse counting SEM. The concentration of tritium in the sample is determined using the radioactive decay equation from the the storage time and the amount of ${ }^{3} \mathrm{He}$ measured. Tritium results are expressed in Tritium Units (TU), where

$$
\mathrm{TU}=\frac{\text { Tritium atoms }}{\text { Hydrogen atoms }} \times 10^{18}
$$

\subsubsection{He Flux Gauge Calculations}

In order to calculate the flux of nitrate into the mixed layer due to physical transport, we used the ${ }^{3} \mathrm{He}$ flux gauge approach. The $\delta^{3} \mathrm{He}$ of all the samples from within the mixed layer were averaged for each timepoint in order to yield a monthly time-series of surface $\delta^{3} \mathrm{He}$. The uncertainty of ${ }^{3} \mathrm{He}$ at a particular timepoint is estimated from the standard error of the mean of the measurements within the mixed layer. The dynamic solubility equilibrium value of $\delta^{3} \mathrm{He}_{e q}$ for each timepoint was calculated from the solubility value of ${ }^{3} \mathrm{He}$ (Benson and Krause, 1980). Additionally, since in the mixed layer, completely trapped bubbles lower $\delta^{3} \mathrm{He}_{e q}$ (Fuchs et al., 1987) and partially trapped bubbles increase $\delta^{3} \mathrm{He}_{e q}$, we use the one-dimensional upper ocean model described in Chapter 4 of this thesis to calculate the effect on $\delta^{3} \mathrm{He}_{e q}$ from air injection and gas exchange.

The excess of $\delta^{3} \mathrm{He}_{e x}$ over the solubility equilibrium value of $\delta^{3} \mathrm{He}_{e q}$ is then calculated to be

$$
\delta^{3} \mathrm{He}_{e x}=\delta^{3} \mathrm{He}-\delta^{3} \mathrm{He}_{e q}
$$

This excess of ${ }^{3} \mathrm{He}$ results in a net flux of ${ }^{3} \mathrm{He}$ out of the ocean. The flux of ${ }^{3} \mathrm{He}$ is calculated according to the diffusive gas exchange parameterization determined in Chapter 4 of this thesis, which is equivalent to the Wanninkhof (1992) quadratic relationship scaled by a factor of 0.79 . Thus the gas transfer velocity, $k$, is given in $\mathrm{cm} \mathrm{hr}^{-1}$ by

$$
k=0.245 u_{10}^{2}\left(\frac{S c_{H e 3}}{660}\right)^{-\frac{1}{2}}
$$

where $u_{10}$ is the wind speed in $\mathrm{m} \mathrm{s}^{-1}$ measured at $10 \mathrm{~m}$ above the sea surface and $S c_{H e 3}$ is the Schmidt number of ${ }^{3} \mathrm{He}$ (ratio of the kinematic viscosity to the molecular diffusivity of the ${ }^{3} \mathrm{He}$ which was calculated 
by scaling the molecular diffusivity of ${ }^{4} \mathrm{He}$ by the square root of the ratio of their masses $\sqrt{\frac{4}{3}}$ ). The gas transfer velocity was calculated at 12 hour intervals using the QuikSCAT wind record during the two week period preceding and following each mixed layer measurement. These gas transfer velocities were averaged and then multiplied by $\frac{24}{100}$ in order to calculate $k_{\text {mean }}$ in units of $\mathrm{m} \mathrm{d}^{-1}$. We explored the sensitivity of the results to the time period during which the gas transfer velocity was calculated, also using periods of one week, three weeks, and four weeks before and after each measurement.

The flux of ${ }^{3} \mathrm{He}$ out of the water, $F_{\mathrm{He} 3}$ in $\% \mathrm{~m} \mathrm{~d}^{-1}$ is equal to

$$
F_{H e 3}=k_{\text {mean }}\left(\delta^{3} \mathrm{He}-\delta^{3} \mathrm{He}_{e q}\right)=k_{\text {mean }} \cdot \delta^{3} \mathrm{He}_{e x}
$$

The flux calculated with the above equation is too small by approximately $2 \%$ to $3 \%$ because we calculate the flux from $\delta^{3} \mathrm{He}_{e x}$ values directly rather than calculating the flux separately for ${ }^{3} \mathrm{He}$ and ${ }^{4} \mathrm{He}$ atoms. This $2 \%$ to $3 \%$ error is small compared to the total uncertainty in the approach (which is approximately $30 \%$ ).

We next correct this flux for the small amount of ${ }^{3} \mathrm{He}$ producted by in situ decay of tritium within the mixed layer. We then fit a least squares smoothing spline to the monthly, tritium-corrected $F_{H e 3}$ estimates in order to integrate the yearly flux, $\bar{F}_{H e 3}$. This flux should be balanced by an upwelling flux of $\delta^{3} \mathrm{He}_{e x}$, and since $\mathrm{NO}_{3}^{-}$is correlated with $\delta^{3} \mathrm{He}_{e x}$, an upwelling flux of $\mathrm{NO}_{3}^{-}$. The upwelling flux of new $\mathrm{NO}_{3}^{-}$to the euphotic zone, $\bar{F}_{\mathrm{NO} 3}$ is thus estimated to be

$$
\bar{F}_{\mathrm{NO} 3}=s_{\mathrm{NO} 3: \mathrm{He}} \cdot \bar{F}_{\mathrm{He} 3}
$$

where $s_{N O 3: H e}$ is the linear regression between $\mathrm{NO}_{3}^{-}$and $\delta^{3} \mathrm{He}_{e x}$. To calculate $s_{N O 3: H e}$, we use the slope from the least squares linear fit between $\delta^{3} \mathrm{He}_{e x}$ and $\mathrm{NO}_{3}^{-}$from our Bermuda data and from data from the CLIVAR repeat hydrography cruises (WOCE lines A20 and A22). We bin the data within a given density range and then calculate the linear regression. We use the following density ranges: (1) all density surfaces between $26.3 \mathrm{~kg} \mathrm{~m}^{-3}$ and $27 \mathrm{~kg} \mathrm{~m}^{-3}$; (2) density surfaces between $26.3 \mathrm{~kg} \mathrm{~m}^{-3}$ and $26.5 \mathrm{~kg} \mathrm{~m}^{-3}$; and (3) density surfaces between $26.3 \mathrm{~kg} \mathrm{~m}^{-3}$ and $26.6 \mathrm{~kg} \mathrm{~m}^{-3}$. 


\subsubsection{Apparent Oxygen Utilization Rate Calculations}

Export production is calculated from the integrated AOUR profiles. First, we combine tritium and ${ }^{3} \mathrm{He}$ data in order to calculate the ventilation age of the water. Tritium decays to ${ }^{3} \mathrm{He}$ with a half-life of 12.31 years (MacMahon, 2006). At the surface, excess ${ }^{3} \mathrm{He}$ is lost through gas exchange, and thus when water is at the surface, the tritium/helium clock is "zeroed". Once a parcel of water submerges, it is sequestered from the atmosphere, and ${ }^{3} \mathrm{He}$ grows in from tritium decay. Thus, in this simple scheme, the T/He age of the water can be calculated as

$$
\text { T/He age }=\lambda^{-1} \log \left[1+\frac{\left[{ }^{3} H e\right]_{e x}}{\left[{ }^{3} H\right]}\right]
$$

In practice, however, mixing complicates matters considerably. If two water parcels with different tritium and helium concentrations mix, the age of the mixture will not be equal to the weighted average of the ages (Jenkins, 1987). Thus for time-scales of more than a few years, the T/He age is not a valid representation of ventilation age (Jenkins and Clarke, 1976). Here, we reduce the problem by using a simple box model with a constant ventilation rate in order to estimate the ventilation age, $\tau$, from the calculated T/He age (Jenkins, 1980). In future work, we will explore more sophisticated treatments of ventilation age.

The box model calculates the concentration of tritium and excess ${ }^{3} \mathrm{He}$ in the interior given surface concentrations of tritium and ventilation time $\tau$. For the surface concentration of tritium we use a source function obtained by blending the Dreisigacker and Roether source function (from 1950 to 1967) with a function obtained by fitting a smooth curve through observed surface tritium values near Bermuda from 1968 to the 1980s (Doney and Jenkins, 1988) and then to the present. The concentration of tritium in the interior box of the model at a time $t$ is given by

$$
C_{T}(t)=C_{T}(t-1)+\frac{1}{\tau}\left(C s(t)-C_{T}(t-1)\right)-\lambda C_{T}(t-1)
$$

where $C_{T}(t)$ is the concentration of tritium in the interior at given timepoint $t, C s(t)$ is the concentration of tritium in the surface at timepoint $t, \tau$ is the ventilation replacement time in years, and $\lambda$ is the decay constant of tritium. The concentration of tritium is determined by the concentration it was at the last time step (first term) plus the contribution from the surface box modulated by $\tau$ (second term) minus the decay of tritium (third term). 
The concentration of excess ${ }^{3} \mathrm{He}$ in the interior box of the model is given by

$$
H(t)=H(t-1)+\frac{1}{\tau}(0-H(t-1))+\lambda C_{T}(t-1)
$$

where $H(t)$ is the concentration of excess ${ }^{3} \mathrm{He}$ in the interior at a given timepoint $t$ and 0 is the surface concentration of excess ${ }^{3} \mathrm{He}$. We are thus assuming that gas exchange effectively zeros the surface excess ${ }^{3} \mathrm{He}$ concentration.

We run this model with values of $\tau$ ranging from 0 to 500 years. For each run, we start the model at 1950 with steady-state equilibrium pre-anthropogenic tritium and ${ }^{3} \mathrm{He}$ levels consistent with the specific $\tau$ and step forward in time until the model predicts the tritium and ${ }^{3} \mathrm{He}$ concentration for the time at which our data was collected (Figure 5-1). We use the tritium and helium concentrations to calculate the T/He age (according to equation 5.8) of the interior as predicted by the model. We then compare the T/He age calculated directly from the data to that predicted by the model in order to determine the ventilation age $\tau$ of our data point. In practice, we calculate the intersection of the calculated T/He data age with the model's $\mathrm{T} / \mathrm{He}$ age curve and use the $\tau$ which is at the intersection point. In order to estimate the uncertainty of $\tau$, we propagate the measurement uncertainties in the ${ }^{3} \mathrm{He}$ and tritium measurements in order to calculate a range of $\mathrm{T} / \mathrm{He}$ age and then use this range to calculate a range of probable values of $\tau$.

In theory, we could instead have compared the tritium data with the model's tritium or the helium data with the model's helium. If we use the ${ }^{3} \mathrm{He}$ for the data/model comparison, then we predict ventilation ages that are approximately $15 \%$ older than when we use $\mathrm{T} / \mathrm{He}$ age. We cannot in practice use tritium data for the model/data comparison for depths shallower than $900 \mathrm{~m}$, because the tritium data does not intersect with the tritium predicted by our model due to errors in our source function. The source function is for a broad area of the ocean rather than for the specific BATS location. For depths deeper than $900 \mathrm{~m}$, a ventilation age based on tritium is $5 \%$ to $10 \%$ younger than a ventilation age based on $\mathrm{T} / \mathrm{He}$ age. By using the $\mathrm{T} / \mathrm{He}$ age we can take advantage of both tritium and ${ }^{3} \mathrm{He}$ and minimize the effect of errors in our source function.

We then combine this ventilation age estimate with the oxygen data in order to calculate the apparent oxygen utilization rate (AOUR), which is given by

$$
A O U R=\frac{\mathrm{O}_{2 \text { sat }}-\mathrm{O}_{2} \text { meas }}{\tau}
$$


Figure 5-1: Predictions for February 2005 for (a and b) tritium, (c and d) ${ }^{3} \mathrm{He}$, and (e and f) T/He age as a function of replacement time $\tau$ from the box model. Plots (a), (c), and (e) illustrate results with to $\tau \leq$ $300 \mathrm{y}$. The black dashed squares in the plots depict the regions that are shown in detail in plots (b), (d), and (f), which present results for $\tau \leq 30$. The intersection of the T/He age calculated for the data with the $\mathrm{T} / \mathrm{He}$ age calculated from the box model results gives the replacement ventilation time scale for each sample. The results of the model shown here are for February 2005 as an example timepoint in the middle of our time-series. In practice, we calculate $\tau$ for every timepoint of our data.
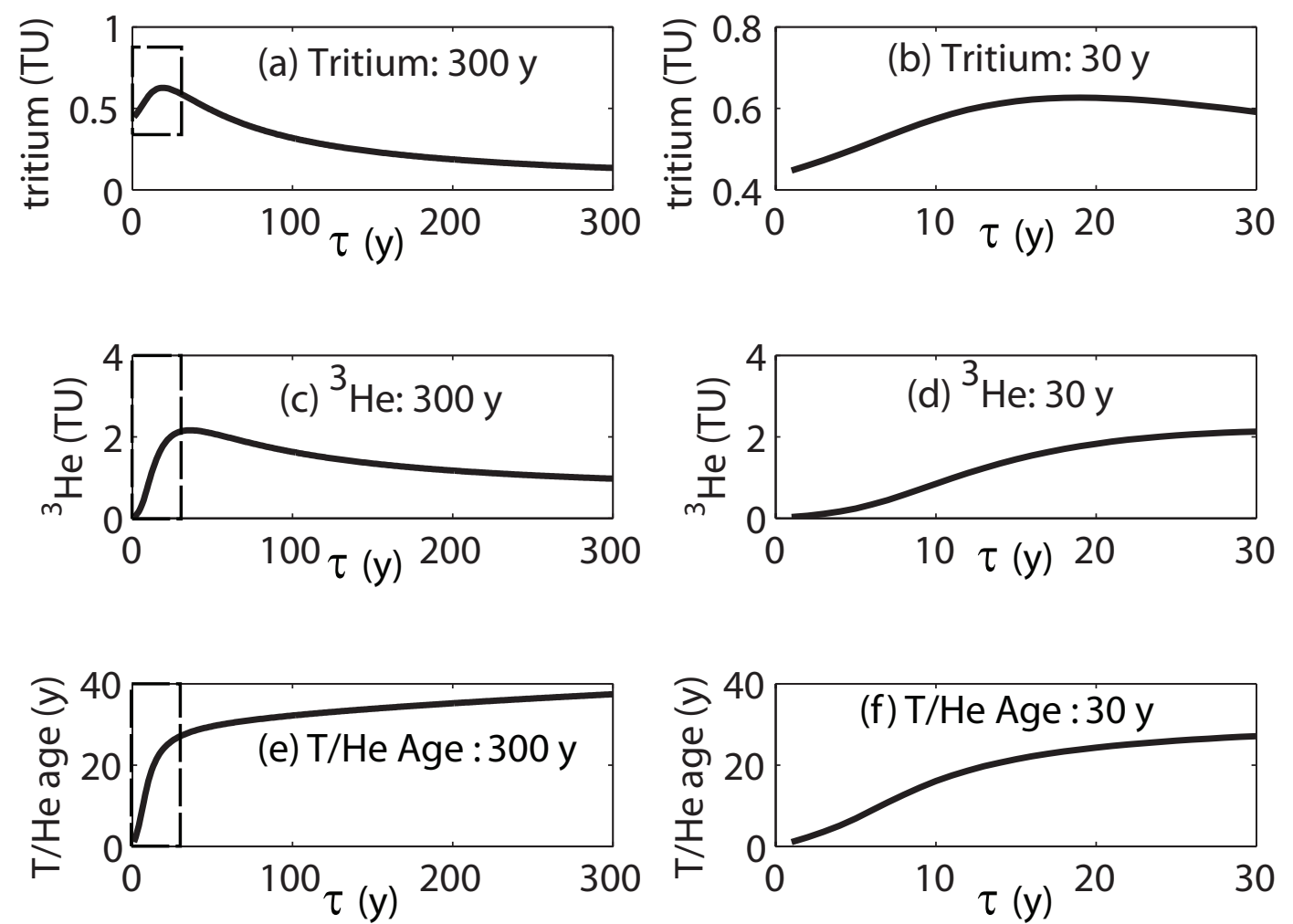
where sat and meas refer to the equilibrium and measured concentrations of $\mathrm{O}_{2}$ respectively. If one assumes that the water was at equilibrium when it was at the surface and that all loss of $\mathrm{O}_{2}$ since then is due to biological consumption, then the AOUR is an estimate of biological consumption and the vertically integrated AOUR is a measure of export production.

\subsubsection{Oxygen and Argon Time-Series Calculations}

Net community production is calculated from euphotic zone seasonal oxygen cycles. The oxygen signal in the euphotic zone is influenced by NCP, gas exchange, thermal forcing, and vertical fluxes of low $\mathrm{O}_{2}$ waters. Here we use the same one dimensional vertical modified Price-Weller-Pinkel (PWP) model that was used in Chapters 3 and 4 of this thesis in order to characterize the thermal forcing, gas exchange fluxes and vertical fluxes. We optimize the model $\mathrm{O}_{2}$ production against depth in order to determine NCP over a seasonal cycle.

The model is described fully in Chapters 3 and 4 of this thesis. In brief, we use a one-dimensional, vertical, modified Price-Weller-Pinkel (PWP) model (Price et al., 1986). The model previously has been extended to include $\mathrm{He}, \mathrm{Ne}, \mathrm{Ar}$, and $\mathrm{O}_{2}$ (Spitzer and Jenkins, 1989) and to include $\mathrm{Kr}$ and $\mathrm{Xe}$ (Stanley et al., 2006). The model is forced with wind stress from the QuikSCAT satellite and with heat fluxes from the National Centers for Environmental Prediction (NCEP) reanalysis (Kistler et al., 2001), interpolated for the BATS site. The surface temperature is restored every 6 hours to a smoothed, interpolated record of the BATS sea surface temperature, with a restoring constant of $75 \mathrm{~W} \mathrm{~m}^{-2} \mathrm{deg} \mathrm{C}-1$. Heat fluxes are added to the model to compensate for the effects of lateral advection and Ekman pumping. Tunable physical parameters include the amount of heat added to the model $(E \mathrm{~km})$, the depth to which it is distributed $(Z)$, and the turbulent vertical diffusivity $K_{z}$ below the mixed layer. These physical parameters work in concert, and ensemble runs were used to choose the three best sets of these physical parameters. The same physical parameters are used here as were used in the base case of Chapter 4 of this thesis. For these studies, the model was spun up from January, 2003 with initial conditions of temperature, salinity, and oxygen profiles from BATS data.

The gas exchange equations used in the model are described in detail in Chapter 3 of this thesis. Therefore here we limit our description to the representation of biological production in the model. Biological production is incorporated through the use of an idealized oxygen production and consumption profile. The 
compensation depth is assumed to be $76 \mathrm{~m}$ for most runs (Najjar and Keeling, 1997; Jin et al., 2007). Above the compensation depth, the oxygen productivity profile, Prod, is represented by a sine curve. A sine was chosen because production should go to low values at the surface due to photo-inhibition and should go smoothly to zero at the compensation depth due to light limitation. Future work will involve exploring the sensitivity of the results to other shapes of productivity profile. The oxygen productivity, Prod is equal to

$$
\operatorname{Prod}=P \cdot \frac{\pi}{2 z_{o}} \cdot \sin \left(\frac{\pi z}{z_{o}}\right) \quad \text { for } \quad z<z_{o}
$$

where $P$ is a tunable model parameter scaling the total amount of production (in $\mathrm{mol}_{2} \mathrm{~m}^{-2} \mathrm{y}^{-1}$ ), $z_{o}$ is the compensation depth $(\mathrm{m})$, the constant $\frac{\pi}{2 z_{o}}$ is used for scaling purposes and is equal to the inverse of the total area under the production curve, and $z$ is depth $(\mathrm{m})$. We examine the sensitivity of our results to the choice of compensation depth. For the base case, we use $z_{o}=76$ and for a sensitivity test, we use $z_{o}=100$.

Below $76 \mathrm{~m}$, oxygen is consumed. We use the profile of AOUR determined in this study as the basis for the shape of $\mathrm{O}_{2}$ consumption in our model (Figure 5-2). We then multiply the consumption profile by $C$, a tunable model parameter controlling the amount of consumption in the model, and by a scaling factor equal to the inverse of the total area under the consumption curve. The AOUR profile shows that consumption persists to $900 \mathrm{~m} . \mathrm{O}_{2}$ in the euphotic zone cannot constrain this deep consumption and thus the value of $C$ is not meant to signify the actual amount of export production in the ocean. Rather, it is only used to provide a reasonable lower boundary condition of $\mathrm{O}_{2}$ so that the model can accurately estimate NCP.

The total oxygen production and consumption, $P_{T O T}$, is then seasonally modulated according to:

$$
P_{T O T}=[1+a \sin (2 \pi(t-t o))] \cdot \operatorname{Prod}
$$

where $a$ is the amplitude of the seasonal modulation, $t$ is time, and to is the seasonal phase. Most runs are performed with $a=1$, and thus with maximum NCP in the summer and zero NCP in the winter (Figure 5-3). We examined the sensitivity of our results to $a=0.75$ and $a=0.5$, which have more production in the winter and thus a less steep seasonal difference. Although primary production peaks earlier in BATS, net community production has been estimated to peak in June (Gruber et al., 1998), and thus our base case run uses $t o=0.2$. We examined the sensitivity of our results to the time of the peak of production by using $t o=0.1$, 
Figure 5-2: The vertical productivity profile used in the model is plotted in arbitrary units. The dashed line indicates division between production and consumption. In the upper $76 \mathrm{~m}$, biological production is modeled as a sinusoidal function, with integrated area equal to tunable model parameter $P$. Below $76 \mathrm{~m}$, biological consumption is modeled according to the shape of the AOUR profile determined as part of this work. The integrated area of the consumption is equal to tunable model parameter $C$. Because we are using euphotic zone $\mathrm{O}_{2}$ data to constrain production, we cannot constrain $C$ well. Instead the consumption is used solely to provide reasonable boundary conditions for $\mathrm{O}_{2}$ below the euphotic zone.

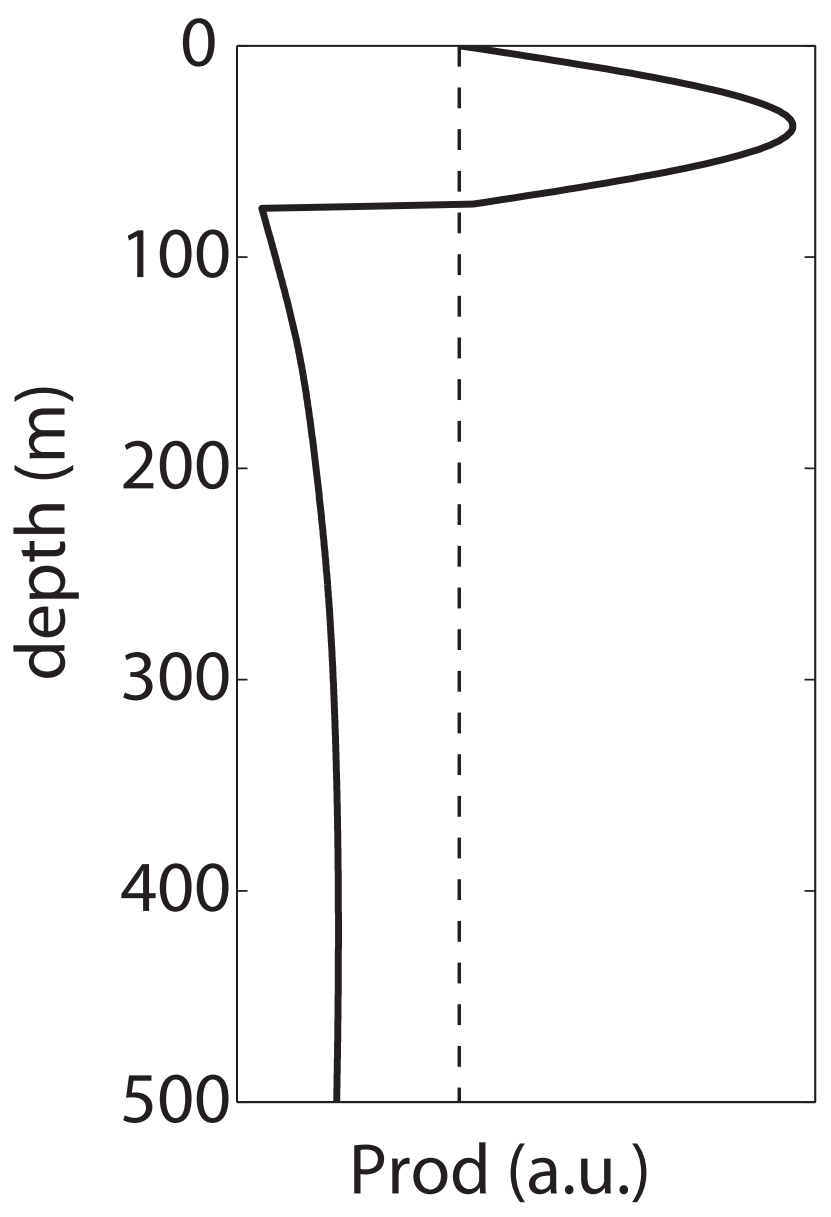


0.2 , and 0.3 , and thus by shifting the peak in production forward and backward by one to two months.

We then use a constrained, non-linear optimization scheme to inversely determine the best values for the parameters $P$ and $C$ and thus to estimate the size of net community production. By using a cost function that compares the difference in $\mathrm{O}_{2}$ and Ar saturation anomalies between the model and the data, we minimize uncertainties associated with thermal forcing and gas exchange representation in the model. The cost function, $C F$, is equal to

$$
C F=\frac{1}{N} \sum_{i=1}^{N}\left[\left(\Delta O_{2}-\Delta A r\right)_{\text {model }}-\left(\Delta O_{2}-\Delta A r\right)_{\text {data }}\right]
$$

where $i$ is a counter for the number of measurements, and $N$ is the total number of measurements included in the cost function. For our base run, we do a point to point comparison of saturation anomalies in the upper $160 \mathrm{~m}$ of the ocean. We examine the sensitivity of our results to the "cut-off" depth of measurements that we include in the cost function by also using cut-off depths of $100 \mathrm{~m}, 120 \mathrm{~m}, 140 \mathrm{~m}$, and $200 \mathrm{~m}$.

\subsection{Results and Discussion}

Three types of biological production are estimated in this study using three different techniques. We first present results and discuss the uncertainties in the new production estimates from the ${ }^{3} \mathrm{He}$ flux gauge method. Next, we present results and discuss the export production estimate from AOUR. Finally, we present results and discuss the net community production estimate from the euphotic zone seasonal cycles of $\mathrm{O}_{2}$ and $\mathrm{Ar}$.

\subsubsection{He Flux Gauge}

The $\delta^{3} \mathrm{He}$ data, gas transfer velocity $k$, and $F_{H e 3}$ estimates are shown in Figure 5-4. The error bars on the data reflect the standard error of the mean of the multiple measurements within the mixed layer. The error is smaller in the winter when there is a deeper mixed layer and thus more measurements within the mixed layer. For all but 3 months, $\delta^{3} \mathrm{He}_{e x}$ is significantly above the dynamic solubility equilibrium value, resulting in a flux of excess ${ }^{3} \mathrm{He}$ out of the water (Figure 5-4a). The gas transfer velocity is larger in the winter due to the stronger winds that occur then (Figure 5-4b). The total flux of excess ${ }^{3} \mathrm{He}$ out of the water peaks in the winter as well (Figure 5-4c). In the winter, deeper mixed layers "mine" the thermocline for excess ${ }^{3} \mathrm{He}$ 
Figure 5-3: Productivity in the model is seasonally modulated according to a sinusoidal function. The base case uses a function with maximum net community production in June and zero production in the winter. We explored the sensitivity of the results to changing the amplitude $a$ of production so that there is a shallower seasonal difference (dashed gray lines) and to shifting the production maximum forward (black dashed line) and backwards (not shown) in time.

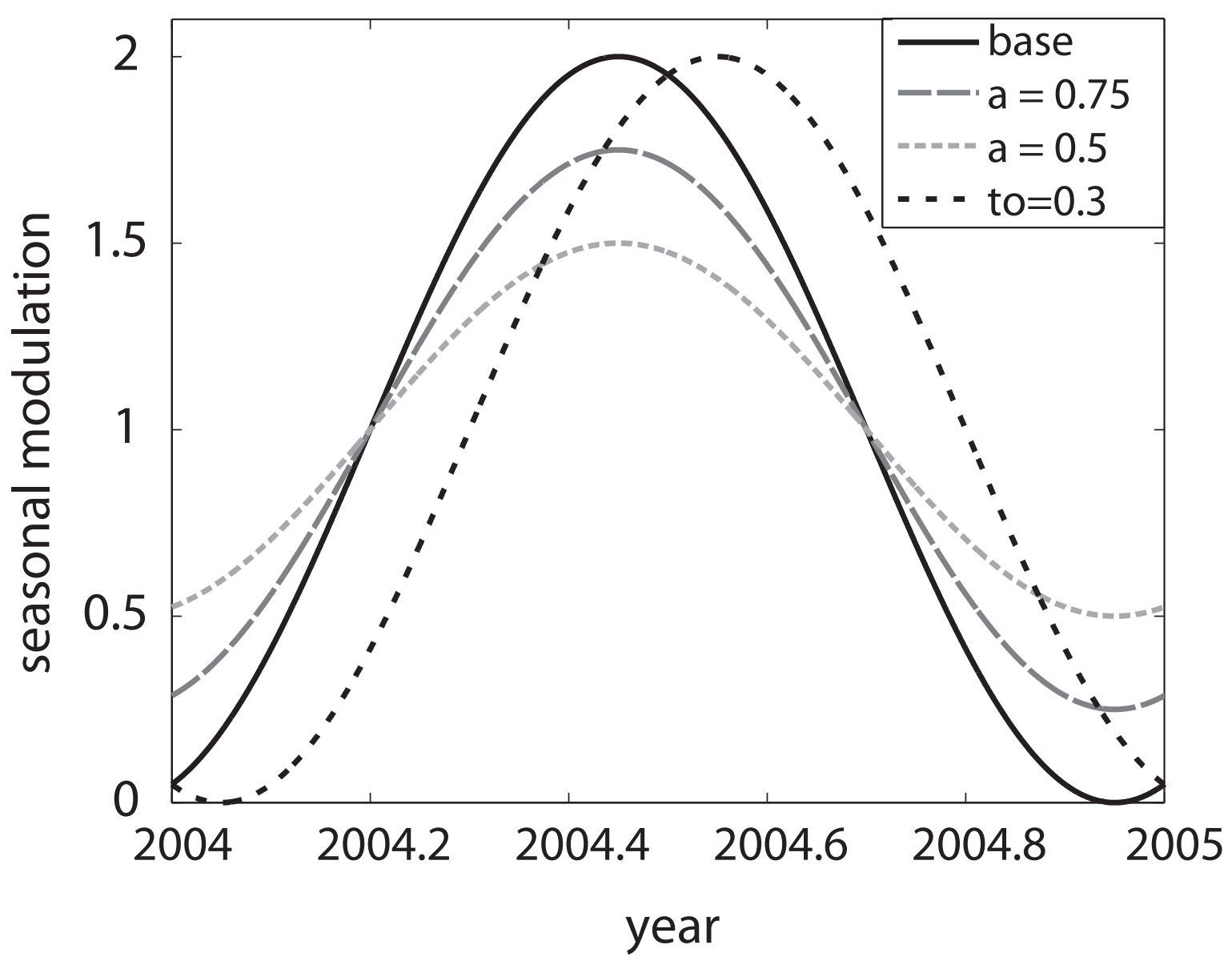


and presumably for nutrients as well. Since a flux into the mixed layer must be balanced by a flux out of the mixed layer, this increased influx of excess ${ }^{3} \mathrm{He}$ results in a larger outflux of excess ${ }^{3} \mathrm{He}$ through air-sea gas exchange.

We fit the excess ${ }^{3} \mathrm{He}$ flux estimates, $F_{H e 3}$, with a least squares smoothing spline. The time average of the spline for 12 months (Sept 2004 to Aug 2005) is a measure of the average flux of excess ${ }^{3} \mathrm{He}$ out of the mixed layer and is equal to $1.37 \pm 0.16 \% \mathrm{~m} \mathrm{~d}^{-1}$. We next correct this flux for the excess ${ }^{3} \mathrm{He}$ in the mixed layer produced by in situ decay of tritium. The mean concentration of tritium in the upper $300 \mathrm{~m}$ (maximum winter mixed layer depth) of the water column at BATS during the one year time-series is $0.73 \pm 0.06 \mathrm{TU}$ (average of 146 samples). The number of ${ }^{3} \mathrm{He}$ atoms produced by this tritium is given by $N \lambda \Delta T$ where $N$ is the number of tritium atoms, $\lambda$ is the decay constant for tritium and is equal to $0.05527^{-1}$, and $\Delta T$ is the change in time over which ${ }^{3} \mathrm{He}$ is being produced. The contribution of tritium decay to the flux of excess ${ }^{3} \mathrm{He}$ is only $0.15 \% \mathrm{~m} \mathrm{day}^{-1}$, or $10 \%$ of our gas exchange flux. Thus the corrected annual average $\bar{F}_{\mathrm{He} 3}$ is $1.22 \pm 0.16 \% \mathrm{~m} \mathrm{day}^{-1}$.

The correlation of $\mathrm{NO}_{3}^{-}$with $\delta^{3} \mathrm{He}_{e x}$ was calculated on a number of different density surfaces in the thermocline, using data from BATS as well as data from the two CLIVAR repeat lines collected in 2003 in the subtropical gyre (Table 5.1). As water ages, it increases in $\delta^{3} \mathrm{He}_{e x}$ due to increased ingrowth of ${ }^{3} \mathrm{He}$ from tritium decay and it increases in $\mathrm{NO}_{3}^{-}$due to increased remineralization. Figure 5-5 shows the correlation for all density surfaces $\sigma_{\theta}<27.0 \mathrm{~kg} \mathrm{~m}^{-3}$ for only Bermuda and for all the subtropical gyre data. The relationships are not significantly different, reinforcing the suitability of this approach. In surface water, however, ${ }^{3} \mathrm{He}_{e x}$ is decoupled from $\mathrm{NO}_{3}^{-}$because ${ }^{3} \mathrm{He}$ is lost in the mixed layer through gas exchange and $\mathrm{NO}_{3}^{-}$is lost in the euphotic zone by biological consumption. Thus we used $\sigma_{\theta}=26.3 \mathrm{~kg} \mathrm{~m}^{-3}$ (about $200 \mathrm{~m}$ ) as the shallowest depth for the regressions. The average slope as determined from the different subsets of data is $1.98 \pm 0.2 \mu \mathrm{mol} \mathrm{N} \mathrm{kg}^{-1} \%^{-1}$ and we use this as our value of $s_{\mathrm{NO}} \mathrm{He}$.

Combining the regression coefficient and the excess ${ }^{3} \mathrm{He}$ flux yields an estimate of $0.90 \pm 0.3 \mathrm{~mol} \mathrm{~N} \mathrm{~m}{ }^{3}$ $\mathrm{y}^{-1}$. The estimated uncertainty includes contributions from measurement error, uncertainty in gas transfer velocity parameterization, uncertainty in solubility equilibrium values, and uncertainty in the $\mathrm{NO}_{3}^{-}: \delta^{3} \mathrm{He}_{e x}$ regression. To compare this with the other estimates of biological production determined here (Table 5.2), we use the revised Redfield ratios of Anderson and Sarmiento (1994) to convert this to an oxygen flux of 9.6 
Figure 5-4: (a) The isotopic ratio anomaly of ${ }^{3} \mathrm{He}$ in the mixed layer (o). Error bars reflect the standard error of the mean of multiple measurements within the mixed layer. The dynamic solubility equilibrium for ${ }^{3} \mathrm{He}$ (-) is calculated using an upper ocean model that includes bubble dynamics. (b) The average gas transfer velocity for ${ }^{3} \mathrm{He}$ calculated during \pm 2 weeks of the ${ }^{3} \mathrm{He}$ measurement. Error bars reflect the $10 \%$ estimated uncertainty in the gas exchange parameterization. (c) The excess ${ }^{3} \mathrm{He}$ flux out of the mixed layer. Error bars reflect the combined error of the measurement uncertainty and the gas transfer velocity uncertainty. Note the flux peaks in the winter when upwelling "mines" the deep water, bringing up ${ }^{3} \mathrm{He}$. The spline that is fit to the data is used to calculate the annual integrated flux.
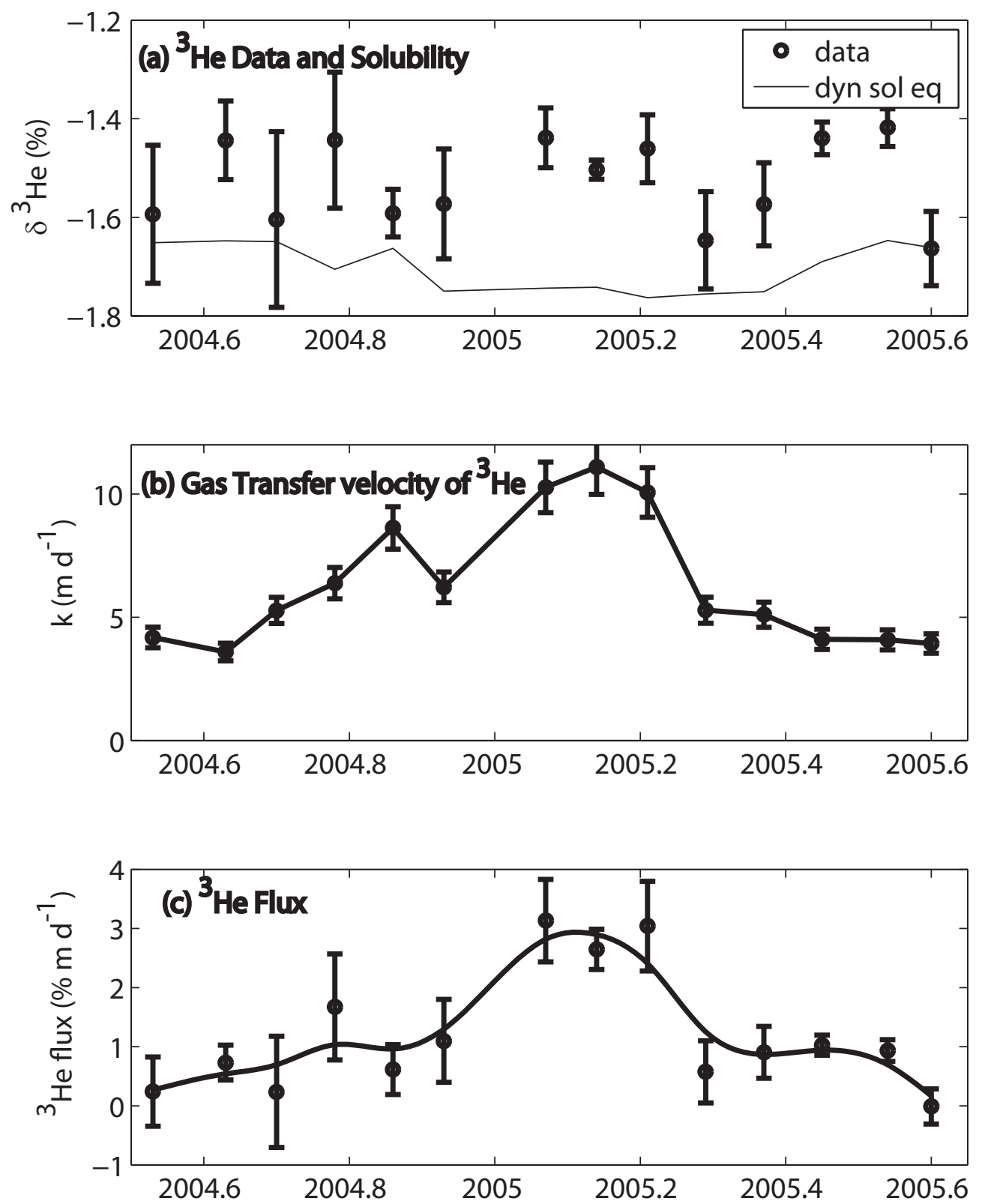
Table 5.1: Regression between $\delta^{3} \mathrm{He}_{e x}$ and $\mathrm{NO}_{3}^{-}$calculated using different subsets of data and different density surfaces (min. density $<\sigma_{\theta}<\max$. density). Slope and intercept are calculated from a linear least squares fit to a plot of the $\mathrm{NO}_{3}^{-}$vs $\delta^{3} \mathrm{He}_{e x}$ data. Bda refers to data collected at the BATS site as part of this study. RH2 and RH3 refer to data collected in 2003 between $20^{\circ} \mathrm{N}$ and $38^{\circ} \mathrm{N}$ as part of the CLIVAR repeat hydrography program along WOCE lines A20 and A22. All slopes are expressed in units of $\mu \mathrm{mol} \mathrm{N} \mathrm{kg}-1$ $\%^{-1}$ and intercepts are in units of $\mu \mathrm{mol} \mathrm{N} \mathrm{kg}^{-1}$.

\begin{tabular}{ccccc}
\hline Data Set & min. density & max.density & Slope & Intercept \\
\hline Bda & 26.30 & 27.00 & $1.98 \pm 0.05$ & $1.39 \pm 0.1$ \\
Bda & 26.30 & 26.60 & $1.94 \pm 0.1$ & $1.45 \pm 0.2$ \\
Bda & 26.30 & 26.50 & $2.08 \pm 0.2$ & $1.32 \pm 0.2$ \\
RH2 & 26.30 & 27.00 & $1.78 \pm 0.08$ & $1.55 \pm 0.3$ \\
RH2 & 26.30 & 26.60 & $1.98 \pm 0.3$ & $0.91 \pm 0.6$ \\
RH2 & 26.30 & 26.50 & $2.49 \pm 0.4$ & $-0.11 \pm 0.7$ \\
RH3 & 26.30 & 27.00 & $1.99 \pm 0.1$ & $0.86 \pm 0.5$ \\
RH3 & 26.30 & 26.60 & $2.03 \pm 0.1$ & $0.66 \pm 0.3$ \\
RH3 & 26.30 & 26.50 & $1.97 \pm 0.12$ & $0.76 \pm 0.4$ \\
All & 26.30 & 27.00 & $1.90 \pm 0.04$ & $1.33 \pm 0.2$ \\
All & 26.30 & 26.60 & $1.80 \pm 0.07$ & $1.42 \pm 0.1$ \\
All & 26.30 & 26.50 & $1.77 \pm 0.1$ & $1.44 \pm 0.2$ \\
& & & & 1.08 \\
& & average & 1.98 & 0.5 \\
& & std & 0.2 & \\
\hline & & & & \\
\hline
\end{tabular}


Figure 5-5: The relationship between $\delta^{3} \mathrm{He}_{e x}$ and nitrate in the thermocline in (a)Bermuda (BATS site) in 2004-2005 and (b) the subtropical gyre in 2003. The red points show data from isopycnal surfaces shallower than $26.3 \mathrm{~kg} \mathrm{~m}^{-3}$. In this region, $\delta^{3} \mathrm{He}_{e x}$ and nitrate are decoupled because nitrate is consumed by biological consumption in the euphotic zone whereas $\delta^{3} \mathrm{He}_{e x}$ is lost through gas exchange in the mixed layer. The blue circles show data from isopycnal surfaces between $26.3 \mathrm{~kg} \mathrm{~m}^{-3}$ and $26.5 \mathrm{~kg} \mathrm{~m}^{-3}$ and the green circles show data from isopycnal surfaces between $26.5 \mathrm{~kg} \mathrm{~m}^{-3}$ and $27 \mathrm{~kg} \mathrm{~m}^{-3}$. The black line is the linear regression for all samples from isopycnal surfaces $26.3 \mathrm{~kg} \mathrm{~m}^{-3}$ to $27 \mathrm{~kg} \mathrm{~m}^{-3}$, and has a slope as marked in units of $\mu \mathrm{mol} \mathrm{N} \mathrm{kg}{ }^{-1} \%^{-1}$. In the text, we also present the regressions for the separate isopycnal surfaces. Data from the subtropical gyre is courtesy of William Jenkins (personal communication).

(a) Bermuda

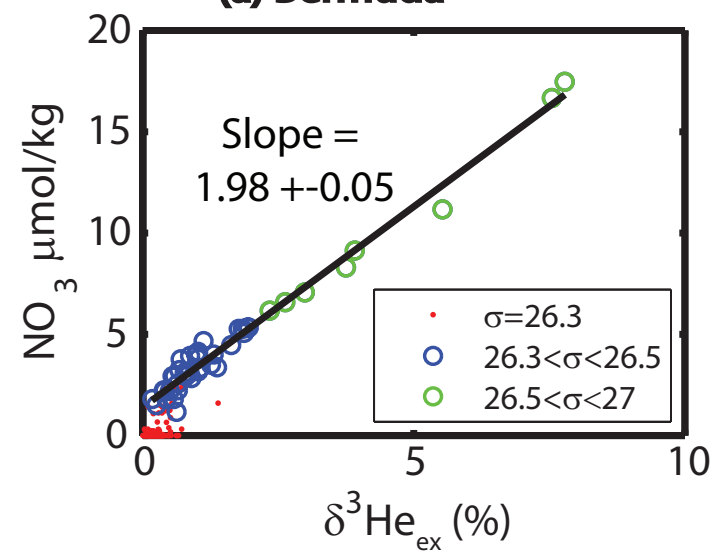

(b) Subtropical Gyre

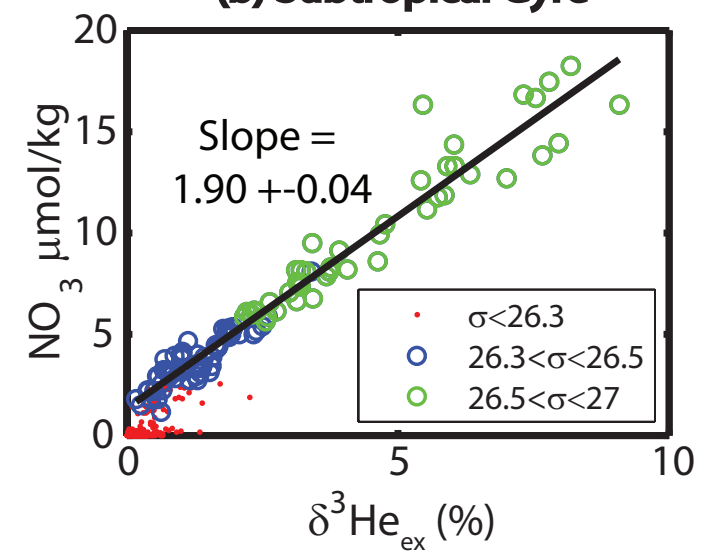


Table 5.2: Estimates of biological production from this study. Fluxes have all been converted into $\mathrm{O}_{2}$ currency using the revised Redfield ratios of Anderson and Sarmiento (1994).

\begin{tabular}{ccc}
\hline Type of Production & Method & Flux $\left(\mathrm{O}_{2} \mathrm{~mol} \mathrm{~m}^{2} \mathrm{y}^{-1}\right)$ \\
\hline New Production & He Flux Gauge & $9.6 \pm 3$ \\
Export Production & AOUR & 3.7 to 5.0 \\
Net Community Production & O2 and Ar time-series & 3.0 to 5.2 \\
\hline
\end{tabular}

$\pm 3 \mathrm{~mol} \mathrm{O}_{2} \mathrm{~m}^{-2} \mathrm{y}^{-1}$ and a carbon flux of $6.6 \pm 2 \mathrm{~mol} \mathrm{C} \mathrm{m}^{-2} \mathrm{y}^{-1}$. The conversion into $\mathrm{O}_{2}$ or $\mathrm{C}$ "currency" might be underestimating the actual production due to the preferential remineralization of $\mathrm{N}$ and $\mathrm{P}$ over $\mathrm{C}$ (Anderson and Pondaven, 2003) and the non-Redfield C/N production of organic matter (Sambrotto et al., 1993) that has been observed at BATS.

The flux of $\mathrm{NO}_{3}^{-}$calculated from this method reflects an estimate of the annual new production as delivered by the physical input of new $\mathrm{NO}_{3}^{-}$to the euphotic zone. It does not take into account nitrogen fixation. Nitrogen fixation, however, is estimated to be only a small fraction of the new production at BATS (Orcutt et al., 2001; Hansell et al., 2004; Knapp et al., 2005) and thus this omission does not contribute to much error in the estimate of new production. Instead, the largest source of uncertainty in the ${ }^{3} \mathrm{He}$ flux gauge estimate of new production stems from the interpretation of the air-sea ${ }^{3} \mathrm{He}$ flux and the ${ }^{3} \mathrm{He}$ and $\mathrm{NO}_{3}^{-}$ regression.

This approach relies on regressing excess ${ }^{3} \mathrm{He}$ with $\mathrm{NO}_{3}^{-}$in the thermocline in order to calculate the $\mathrm{NO}_{3}^{-}$flux into the euphotic zone. By integrating the $\delta^{3} H e_{e x}$ profile, we can calculate that the annual integrated gas exchange flux of excess ${ }^{3} \mathrm{He}$ is equal to the excess ${ }^{3} \mathrm{He}$ inventory in the upper $500 \mathrm{~m}$ of the water column, and hence using a regression in the thermocline seems appropriate. However, this regression is not straightforward, given that there is a non-zero intercept (Table 5.1). This non-zero intercept may be the result of a steeper relationship in the upper waters because $\mathrm{NO}_{3}^{-}$is being remineralized in the shallow aphotic zone whereas ${ }^{3} \mathrm{He}$ is not. It may also be a result of winter deep mixed layers mining ${ }^{3} \mathrm{He}$ and through gas exchange fluxing it out at a time when biological uptake of $\mathrm{NO}_{3}^{-}$is small.

The implications of this non-zero intercept is that the amount of $\mathrm{NO}_{3}^{-}$brought up with the ${ }^{3} \mathrm{He}$ varies 
depending upon which depth the water is coming from. We cannot determine which depth the ${ }^{3} \mathrm{He}$ is coming from and thus we do not know which $\mathrm{NO}_{3}^{-}: \delta^{3} \mathrm{He}_{e x}$ ratio to use. Thus we are using as a conservative estimate the slope of the relationship, but this may be an underestimate of the actual $\mathrm{NO}_{3}^{-}$brought up, especially if the $\mathrm{NO}_{3}^{-}$comes from the shallow waters of the upper thermocline where remineralization is largest. In some sense, this method is defining new production as production that is a result of nitrate coming from far away rather than from the seasonal cycles of $\mathrm{NO}_{3}^{-}$remineralized in the upper thermocline. One might expect, then, that our estimate of new production from this method would be on the low side of production estimates; surprisingly, it is significantly larger than the estimates of production from AOUR and euphotic zone oxygen cycles (Table 5.2).

Another issue is that the correlation between excess ${ }^{3} \mathrm{He}$ and $\mathrm{NO}_{3}^{-}$in the thermocline is changing with time since the ${ }^{3} \mathrm{He}$ distribution is evolving while the $\mathrm{NO}_{3}^{-}$distribution is essentially constant (Fig. 5-6). A decade ago, the slope between ${ }^{3} \mathrm{He}$ and nitrate was $\sim 0.95 \mu \mathrm{mol} \mathrm{N} \mathrm{kg}{ }^{-1} \%{ }^{-1}$ rather than the value of 1.98 $\mu$ mol $\mathrm{N} \mathrm{kg}^{-1} \%^{-1}$ used here. The water in the main thermocline that is being accessed for the ${ }^{3} \mathrm{He}$ flux may be several years old and thus perhaps the slope from several years ago should be used instead of the correlation calculated from today's values. Since this older water has a shallower slope, the ${ }^{3} \mathrm{He}$ flux gauge estimate may be an overestimate of the production because it uses the steeper, modern slope.

Additionally, the approach may overestimate new production if some of the water that is transported into the euphotic zone at BATS has been transported from the North where production is higher. Between the base of the euphotic zone and base of the mixed layer, $\mathrm{NO}_{3}^{-}$can be removed from the water without removal of ${ }^{3} \mathrm{He}$. Such water, therefore, would have a less steep $\mathrm{NO}_{3}^{-}:{ }^{3} \mathrm{He}$ correlation than the one we use here, leading to our estimate being an overprediction of new production. The ${ }^{3} \mathrm{He}$ flux gauge technique may therefore yield an estimate of production on the regional spatial scale, rather than on a local spatial scale.

Another uncertainty in this method comes from the estimate of the dynamic solubility equilibrium value $\delta^{3} \mathrm{He}_{e q} . \delta^{3} \mathrm{He}_{e q}$ is a function of temperature and gas dynamics (Fuchs et al., 1987). The solubility equilibrium given only temperature considerations, $\delta^{3} \mathrm{He}_{s o l}$, can be calculated from the relationship of Benson and Krause (1980). To determine the contribution from gas dynamics to $\delta^{3} \mathrm{He}_{e q}$, we added nontritiugenic ${ }^{3} \mathrm{He}$ to the one dimensional vertical mixed layer model described in Chapter 4 of this thesis. We used the parameterizations of air injection (including both complete and partial trapping) and diffusive gas exchange 
Figure 5-6: The correlation between excess ${ }^{3} \mathrm{He}$ and $\mathrm{NO}_{3}^{-}$has changed with time. In 1985-1988 (data marked by $\mathrm{x}$ ), the slope for Bermuda data on the density horizons between $\sigma_{\theta}=26.3 \mathrm{~kg} \mathrm{~m}^{-3}$ and $\sigma_{\theta}=27.0$ $\mathrm{kg} \mathrm{m}^{-3}$ was equal to $0.95 \pm 0.04 \mu \mathrm{mol} \mathrm{N} \mathrm{kg}^{-1} \%^{-1}$ and in 2005 (data marked by o) the slope is equal to $1.98 \pm 0.05 \mu \mathrm{mol} \mathrm{N} \mathrm{kg}{ }^{-1} \%^{-1}$. Only the regression line (dashed) is drawn for data from the 1993 from WOCE line A20 between latitude $20^{\circ} \mathrm{N}$ and $38^{\circ} \mathrm{N}$ which has a slope equal to $1.17 \pm 0.06 \mu \mathrm{mol} \mathrm{N} \mathrm{kg}^{-1}$ $\%^{-1}$. Data from 1985-1988 is from Jenkins and Doney (2003).

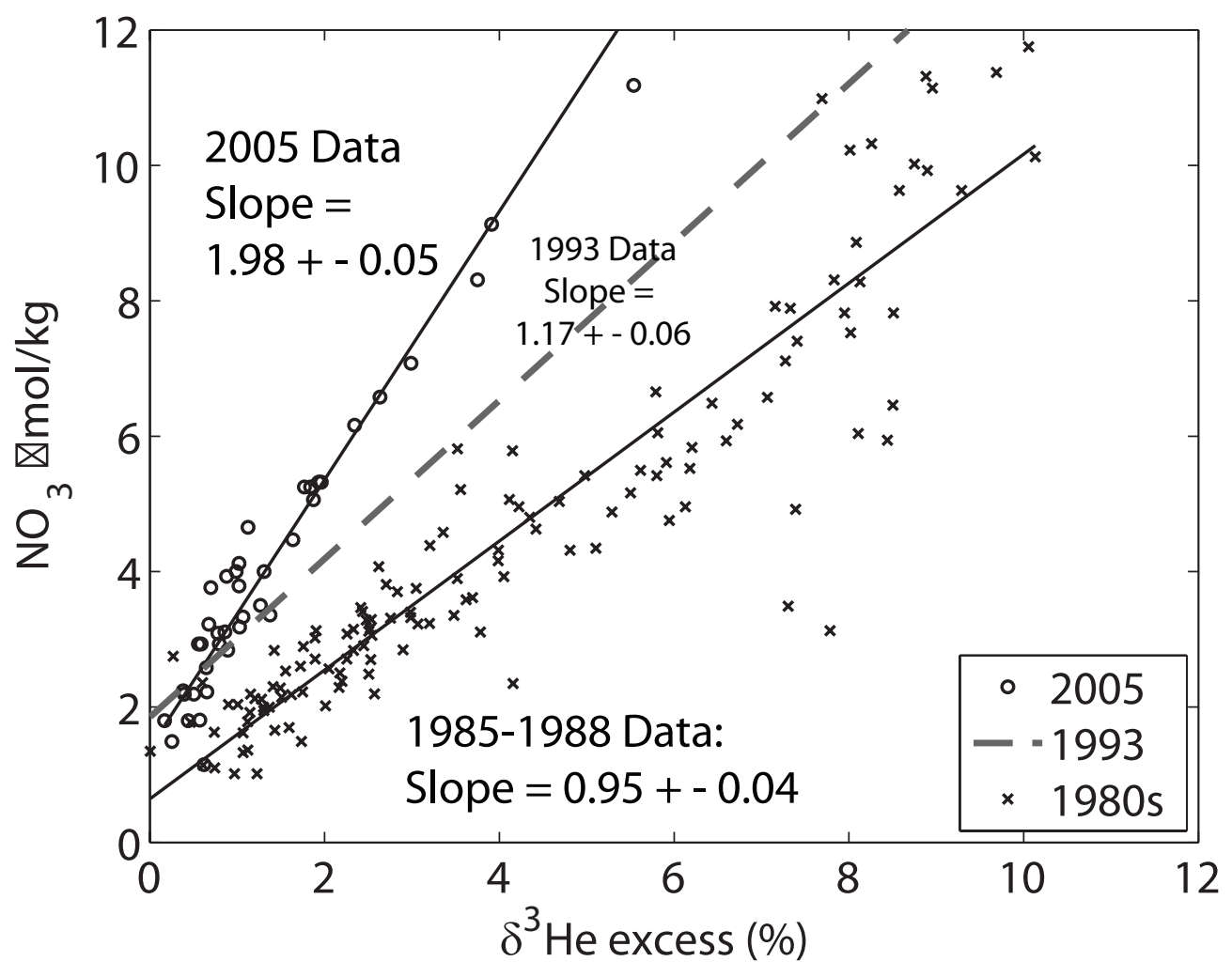


as determined in Chapter 4 by the time-series of noble gases in order to determine $\delta^{3} \mathrm{He}_{e q}$.

In addition, we performed a sensitivity study to see how much $\delta^{3} \mathrm{He}_{e q}$ would change under different air-sea gas exchange parameters (Figure 5-7). In Chapter 4 we explain how depending on the exact form of cost function, we determined a range of air injection and diffusive gas exchange magnitudes. Here we use the parameters from the base case, a set of parameters that yields low air injection fluxes and where the flux has a relatively small contribution from complete trapping and a set of parameters that yields larger air injection fluxes with a larger contribution from complete trapping. For these three cases, $\delta^{3} \mathrm{He}_{e q}$ varies by on average $0.04 \%$. An uncertainty of $0.04 \%$ in $\delta^{3} \mathrm{He}_{e q}$ leads to uncertainties of order $20 \%$ in the new production estimate.

We also examined the effect on $\delta^{3} \mathrm{He}_{e x}$ of having an air injection flux that was entirely due to partially trapped bubbles or one that was entirely due to completely trapped bubbles using the same diffusive gas exchange and total air injection. Neither of these latter two situations would occur in the ocean but they are useful as end-members in order to investigate the total possible range in $\delta^{3} \mathrm{He}_{e x}$. If the bubble flux is all due to complete trapping, then $\delta^{3} \mathrm{He}_{e x}$ decreases by on average $0.04 \%$. A complete bubble flux injects air of atmospheric ratios. In steady-state, the flux in must be balanced by the flux out. Since the gas transfer velocity of ${ }^{3} \mathrm{He}$ is larger than of ${ }^{4} \mathrm{He}$ (because of the larger diffusivity of ${ }^{3} \mathrm{He}$ ), the equilibrium concentration must be lower so that the total gas flux out is the same. If the bubble flux is all due to partial trapping, then $\delta^{3} \mathrm{He}_{e x}$ increases by on average $0.08 \%$. ${ }^{3} \mathrm{He}$ and ${ }^{4} \mathrm{He}$ have almost the same solubility but ${ }^{3} \mathrm{He}$ has a higher diffusivity by a factor of $\sim \sqrt{\frac{4}{3}}$. Diffusive gas exchange is proportional to $D^{\frac{1}{2}}$ whereas air injection flux due to partially trapped bubbles is proportional to $D^{\frac{2}{3}}$. The more diffusive gas, ${ }^{3} \mathrm{He}$ therefore escapes the bubbles more easily and hence the ratio of ${ }^{3} \mathrm{He} /{ }^{4} \mathrm{He}$ in the water is higher, resulting in $\delta^{3} \mathrm{He}_{e q}$ being greater than $\delta^{3} \mathrm{He}_{\text {sol }}$.

The estimate of diffusive gas transfer velocity has uncertainties from two sources. One is from the parameterization used. Here we use the gas exchange parameterization developed in Chapter 4 of this thesis which has an uncertainty of order $10 \%$. The second source is due to the window of wind speeds used to calculate the gas transfer velocity. We performed the above calculations using a \pm two week window around the time of sampling since approximately two weeks is the expected gas exchange time-scale for the mixed layer. We investigated the effect on the results of using a shorter or longer window. If a one week window is 
Figure 5-7: Air injection affects the dynamic solubility equilibrium $\delta^{3} \mathrm{He}_{e q}$ and thus knowledge of the amount of air injection and the partitioning between completely and partially trapped bubbles is necessary. The three solid lines show the $\delta^{3} \mathrm{He}_{e q}$ predicted by our model given the "base case" air injection parameters as determined in Chapter 4 of this thesis. The low air injection and high air injection curves are calculated using the parameters determined in Chapter 4 that gave the smallest and largest air injection fluxes, with small and large fractions of complete trapping respectively, and thus represent the "extreme cases" of reasonable values. The high air injection and the base case give almost the same dynamic solubility equilibrium values. The small air injection case predicts values within $0.04 \%$ of the others. The green dashed line is the equilbrium value based only on temperature. The "end-member" cases of all complete or all partial trapping are shown by the other dashed lines.

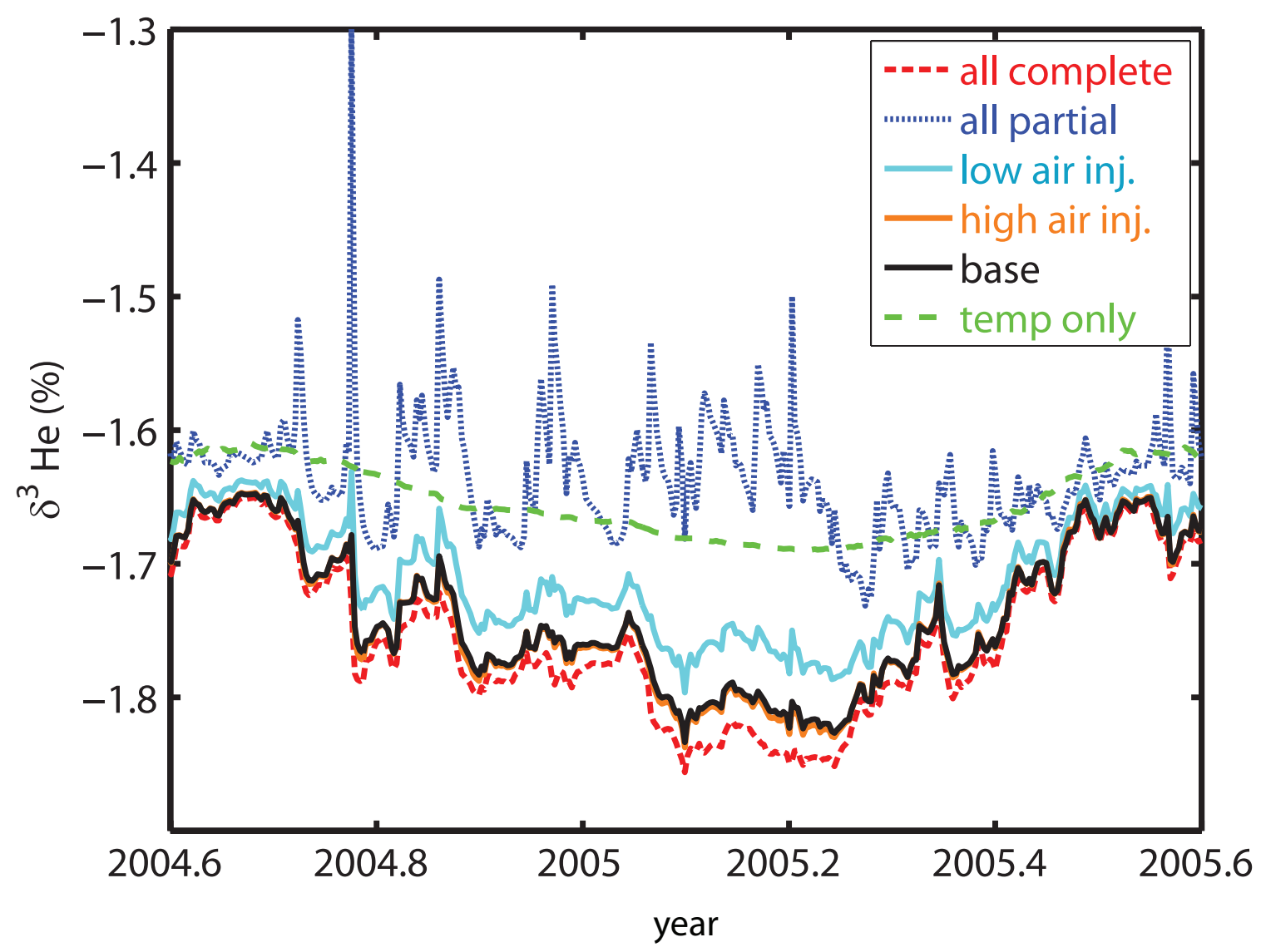


used, then the average gas transfer velocity decreases by $7 \%$, perhaps because of a fair weather bias in the sample collection. If a 3 or 4 week window is used, the average gas transfer velocity decreases by $1 \%$ or $2 \%$ respectively. Thus the results are not very sensitive to the choice of window used and hence we estimate the uncertainty due to gas exchange to be $10 \%$.

The $\mathrm{NO}_{3}^{-}$flux calculated from this time series is $\sim 10 \%$ larger, but not significantly different, than that calculated by Jenkins and Doney (2003). This work differs from that of Jenkins and Doney in several ways. First, their work was based on a five year time-series and thus is less sensitive to interannual variations. Although at the moment we have only used an effectively one year time-series, we have samples from another two years. Second, the data they used was from the 1980s when there was more tritium and ${ }^{3} \mathrm{He}$ in the water column. This is not a serious problem for our study since though ${ }^{3} \mathrm{He}$ has decreased over time, the measurement techniques have improved. Third, they used a gas exchange parameterization with a cubic dependence on wind speed (Wanninkhof and McGillis, 1999) whereas we use a gas exchange parameterization with a quadratic dependence on wind speed. They state that using the gas exchange parameterization of Wanninkhof (1992) yielded slightly higher but significantly similar results. Our diffusive gas exchange parameterization is $20 \%$ lower than that of Wanninkhof (1992) and thus if the only difference were due to gas exchange parameterization, our flux should be lower than that of Jenkins and Doney, not higher. Finally, they calculated the solubility equilibrium value from a gas exchange model which used different parameterizations for air injection and thus they used a $\delta^{3} \mathrm{He}_{e q}$ that was elevated beyond that predicted solely from temperature by $0.06 \%$, whereas we used a $\delta^{3} \mathrm{He}_{e q}$ that was depressed from that predicted solely from temperature by $-0.05 \%$. Since we used a smaller $\delta^{3} \mathrm{He}_{e q}$, we calculated a larger flux of $\delta^{3} \mathrm{He}_{e x}$ and a larger $\mathrm{NO}_{3}^{-}$flux. In the Jenkins and Doney study, $\delta^{3} \mathrm{He}$ was larger (because the time-series was from the 1980s) and thus their results are much less sensitive to $\delta^{3} \mathrm{He}_{e q}$. In spite of the significant differences between the two studies, the results are remarkably concordant.

\subsubsection{Apparent Oxygen Utilization Rates}

The tritium and excess ${ }^{3} \mathrm{He}$ data that are used to calculate the ventilation ages are presented in Figure 5-8. Three full years of tritium data are shown whereas only one year of excess ${ }^{3} \mathrm{He}$ has been measured so far. The tritium data has been decay corrected to 2005. Tritium is highest in the subtropical mode water of the 
main thermocline. In some profiles, there is a second maximum at 1200 to $1600 \mathrm{~m}$. A maximum at about this density of 27.7 has been observed previously (Jenkins, 1980) and has been attributed to a northerly source. Tritium then decreases dramatically below approximately $2400 \mathrm{~m}$.

Excess ${ }^{3} \mathrm{He}$ is at a maximum between 900 and $2000 \mathrm{~m}$ - this is in the region where there is still measurable tritium but is below the main thermocline where ${ }^{3} \mathrm{He}$ can be lost upwards due to mixing and then gas exchange. Below $2000 \mathrm{~m}$, there is significant excess ${ }^{3} \mathrm{He}$ even though the tritium concentration is very low. This water, which has high silica as well as high ${ }^{3} \mathrm{He}$, is probably upper North Atlantic Deep Water that has been influenced by Circumpolar Water (Jenkins and Clarke, 1976). In the Pacific, hydrothermal vents inject primordial ${ }^{3} \mathrm{He}$ into the deep water. This water then flows into the Southern ocean and becomes Circumpolar Intermediate Water, still with a high ${ }^{3} \mathrm{He}$. Some of this Circumpolar Water spins off into the Atlantic, bringing its ${ }^{3} \mathrm{He}$ signature with it. Thus the ${ }^{3} \mathrm{He}$ in the deep water may be from primordial ${ }^{3} \mathrm{He}$ in the Pacific. We can estimate the ${ }^{3} \mathrm{He}$ contribution from such water: We use ${ }^{3} \mathrm{He}$ and Si data from WOCE line SR01 (Drake Passage) to calculate that the regression between Si and excess ${ }^{3} \mathrm{He}$ is $0.031 \mathrm{TU} \mathrm{kg} \mu \mathrm{mol}^{-1}$ $\mathrm{Si}$. We then combine the Si concentrations measured in our deep samples with the regression to calculate that the ${ }^{3} \mathrm{He}$ contribution from the Antarctic in the deep waters is 0.5 to $1.8 \mathrm{TU}$. The remainder of the ${ }^{3} \mathrm{He}$ signal in the deep waters ( 0.5 to $1 \mathrm{TU}$ ) may come from the volcanic activity in the North Mid-Atlantic Ridge along the Gibbs Fracture Zone (Jenkins and Clarke, 1976) or may come from old water in which the natural (pre-bomb) tritium has already completely decayed.

Profiles of ventilation time $\tau$ from the two deep profiles are presented in Figure 5-9. Qualitatively, as depth increases, water ages and thus $\tau$ increases. In the profiles, there is a maximum at approximately $1100 \mathrm{~m}$ in November and at $900 \mathrm{~m}$ in April. These depths correspond to density surfaces of $27.66 \mathrm{~kg}$ $\mathrm{m}^{-3}$ and $27.32 \mathrm{~kg} \mathrm{~m}^{-3}$ respectively. A similar maximum at about $900 \mathrm{~m}$ was observed by Jenkins (1980). This maximum is probably the result of low tritium, high ${ }^{3} \mathrm{He}$ Antarctic Intermediate Water, as $\mathrm{SiO}_{2}$ is at a maximum as well. Our simple box ventilation model breaks down below the main thermocline and therefore we can only use $\tau$ estimates at depths shallower than $\sim 800 \mathrm{~m}$.

The T/He age is also plotted on Figure 5-9 for reference. In the surface, the T/He age agrees well with $\tau$. Between 300 and $800 \mathrm{~m}$, the $\mathrm{T} / \mathrm{He}$ age is an overestimate of the ventilation age. Since the change in decay-corrected tritium concentration is decreasing with time, mixing favors old waters, resulting in the 
Figure 5-8: A time-series of (a) tritium and (b) excess ${ }^{3} \mathrm{He}$ (reported in $\mathrm{TU}$ for ease in comparison with tritium). Only one year of the ${ }^{3} \mathrm{He}$ time-series has been measured, whereas the tritium time-series is for 3 years. Tritium data has been decay-corrected to 2005, a midpoint in the time-series and thus is reported in

$\mathrm{TU}_{05}$. Sample locations are marked by dots. Contours are drawn every $0.1 \mathrm{TU}_{05}$ for tritium and every 0.2 TU for ${ }^{3} \mathrm{He}$.
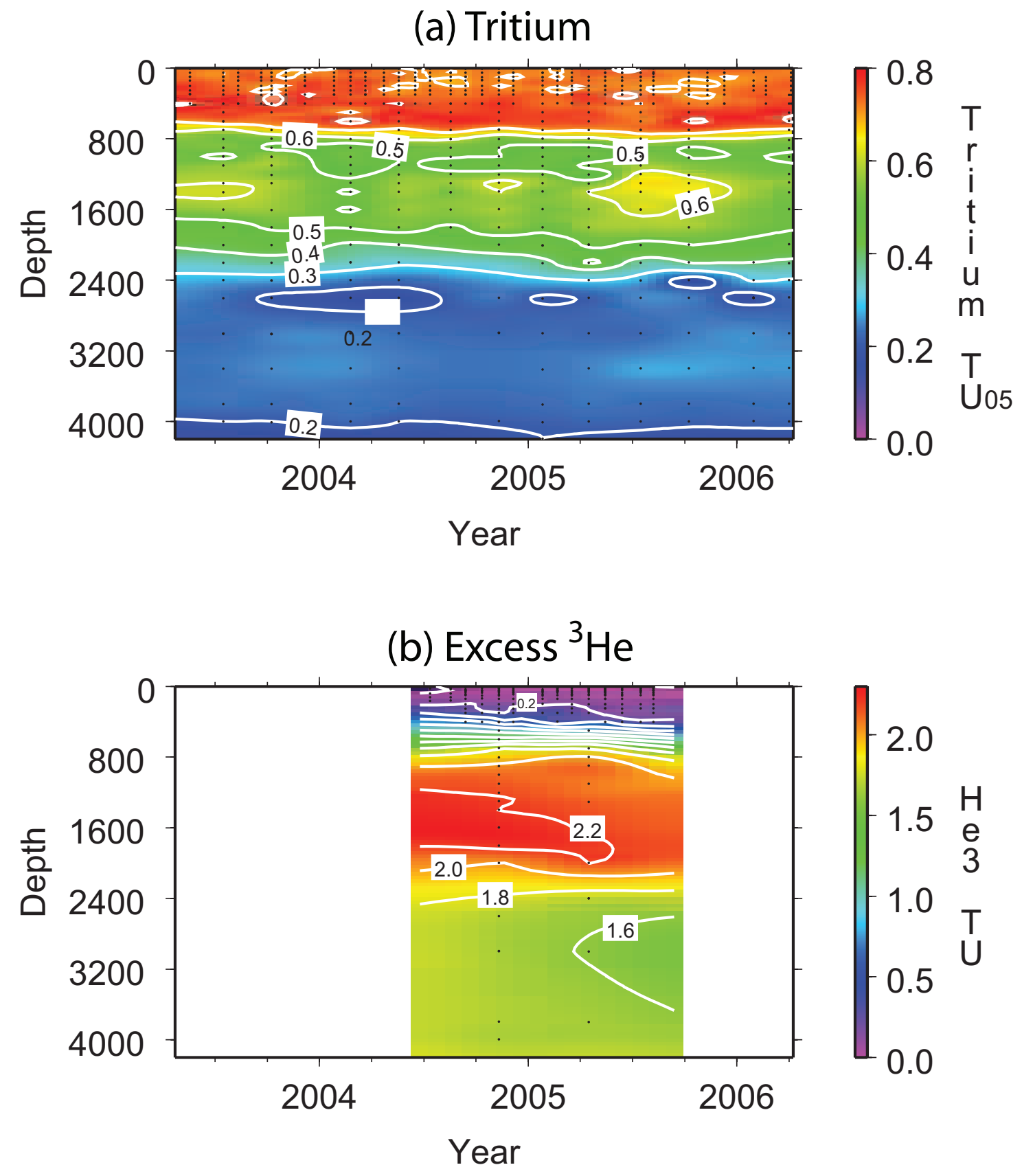
Figure 5-9: Profiles of $\tau$ in (a) the upper $2200 \mathrm{~m}$ and (b) the upper $800 \mathrm{~m}$ of the water column for November (red) and April (blue). Also plotted is T/He age for comparison. The peak in $\tau$ at about 900 or $1100 \mathrm{~m}$ depending upon the month is a sign of lateral advection of a different water mass, suggesting that using the box model is no longer appropriate at those depths.

(a) upper $2200 \mathrm{~m}$

(b) upper $800 \mathrm{~m}$
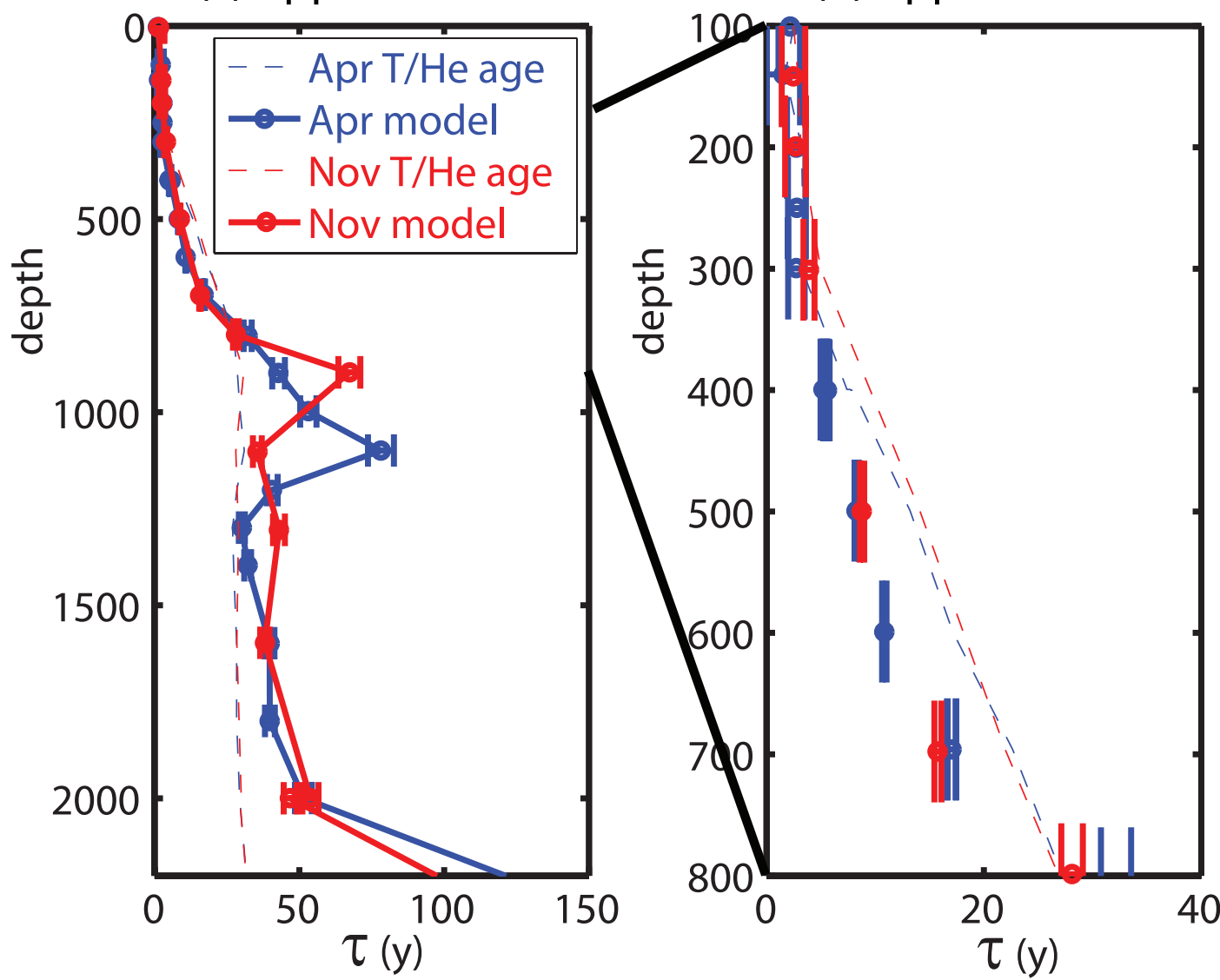
$\mathrm{T} / \mathrm{He}$ age being too high. Below $800 \mathrm{~m}$, the T/He age is smaller than $\tau$, but as described above, we cannot use our box model at these depths and thus the comparison is irrelevant.

We next used $\tau$ to calculate the AOUR (Figure 5-10) for the two deep profiles and for our monthly data at depths between 140 and $400 \mathrm{~m}$. The monthly data shows significant scatter. At these shallower depths, $\tau$ has a steep slope with respect to the T/He age. Hence measurement uncertainties of ${ }^{3} \mathrm{He}$ and tritium lead to large uncertainties in $\tau$ and thus large uncertainties in AOUR. Additionally, there is seasonal and interannual variability in AOU in the shallower depths (Jenkins and Goldman, 1985). Luckily, however, we have fourteen measurements at each of these shallower depths and by averaging all these measurements can compensate for the larger uncertainty. Although we show AOUR estimates for depths down to 2200, for reasons mentioned above, our approach is not valid below approximately $800 \mathrm{~m}$ and thus these deep AOUR estimates cannot be used. Thus the AOUR integral is a lower bound on the export flux.

We are most confident in the AOUR estimates between $200 \mathrm{~m}$ and $800 \mathrm{~m}$. At these depths our approach should give robust value for $\tau$ and we should be well enough below $\mathrm{O}_{2}$ production in the euphotic zone for it not to unduly influence our results. We fit a spline to the data between 200 and $800 \mathrm{~m}$, and by integrating the spline, we calculated the vertically integrated AOUR between 200 and $800 \mathrm{~m}$ to be equal to $3.73 \pm 0.19$ mol O $\mathrm{m}^{-2} \mathrm{y}^{-1}$.

Remineralization is also occurring above $200 \mathrm{~m}$ and below $800 \mathrm{~m}$. At $140 \mathrm{~m}$, there is enough ${ }^{3} \mathrm{He}$ that we can still calculate $\tau$. However, there is increased uncertainty because we are getting uncomfortably close to the $\mathrm{O}_{2}$ compensation depth, and thus $\mathrm{O}_{2}$ may be diffusing downward, confounding our estimates. Therefore our method may be underestimating the total export. Nonetheless, we can still calculate an integrated AOUR for the water column between 140 and $200 \mathrm{~m}$. In order to estimate the contribution of the remineralization in the water column above 140 (below $800 \mathrm{~m}$ ), we "extended" the AOUR estimates by fitting the upper (lower) few data points with a quadratic function. We then integrate the area under these quadratic extensions to get a rough idea of the remineralization in these regions. These numbers are useful in so much as they give us an idea of the contribution of the regions of the water column that are not well constrained by our approach. The estimates of the integrated AOUR, or export production, for each region of the water column are given in Table 5.3. The total export production integrated between $75 \mathrm{~m}$ and $1000 \mathrm{~m}$ is $5.0 \mathrm{~mol} \mathrm{O} 2 \mathrm{~m}-2 \mathrm{y}-1$. From deep sediment traps, we know that there is non-zero export below $1000 \mathrm{~m}$ (Conte et al., 2001) and thus this 
Figure 5-10: Profiles of AOUR in (a) the upper $2200 \mathrm{~m}$ and (b) the upper $800 \mathrm{~m}$ of the water column for all the data (gray circles) and averages at each depth horizon (black circles). For each data point, the error bars reflect the propagated error from the ventilation age estimate. For the depth averages, the error bars reflect the standard error of the mean of all AOUR determinations made at that depth. A spline fit of the data from 140 to $800 \mathrm{~m}$ (black line) was used to determine the integrated AOUR. Below $800 \mathrm{~m}$, the model ventilation age estimates are not reasonable. We show the AOUR estimates below $800 \mathrm{~m}$ but we do not use the data below $800 \mathrm{~m}$ in our integration. Rather, we extend the AOUR curve by fitting a quadratic to the 600 to 800 $\mathrm{m}$ data. Similarly, near the surface our AOUR calculation breaks down and thus we extend the curve upward by fitting a quadratic curve to the 140 to $300 \mathrm{~m}$ data (dashed line).

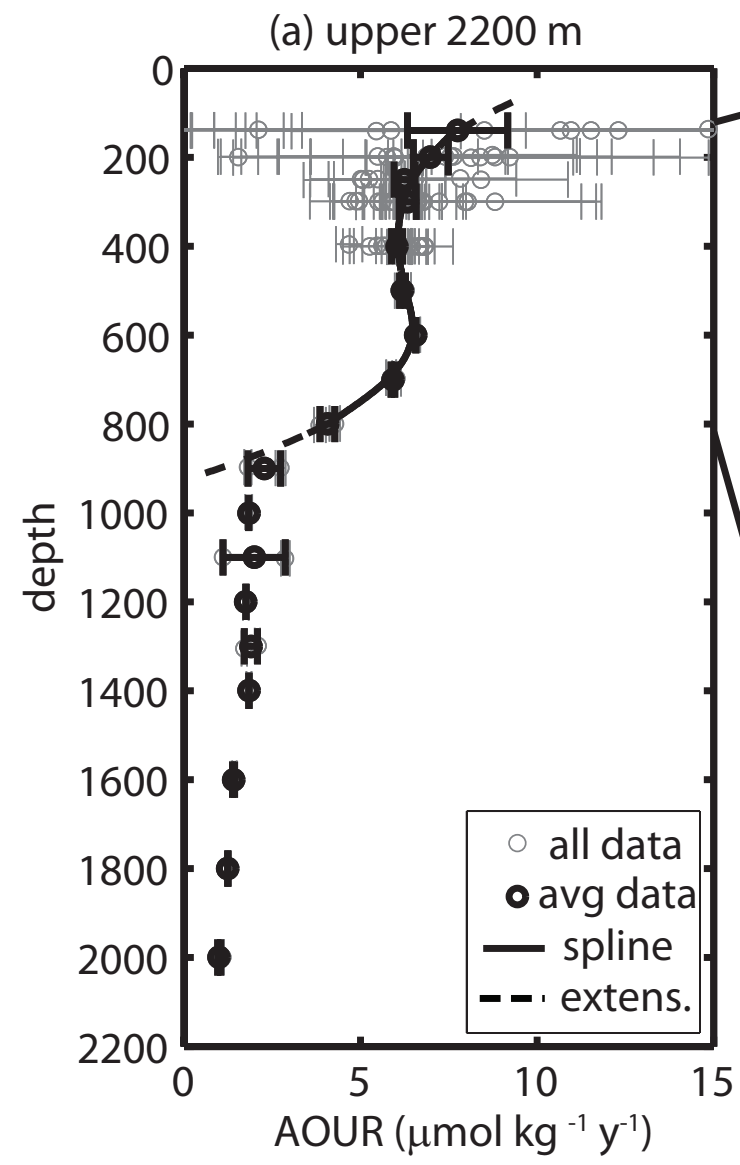

(b) upper $800 \mathrm{~m}$

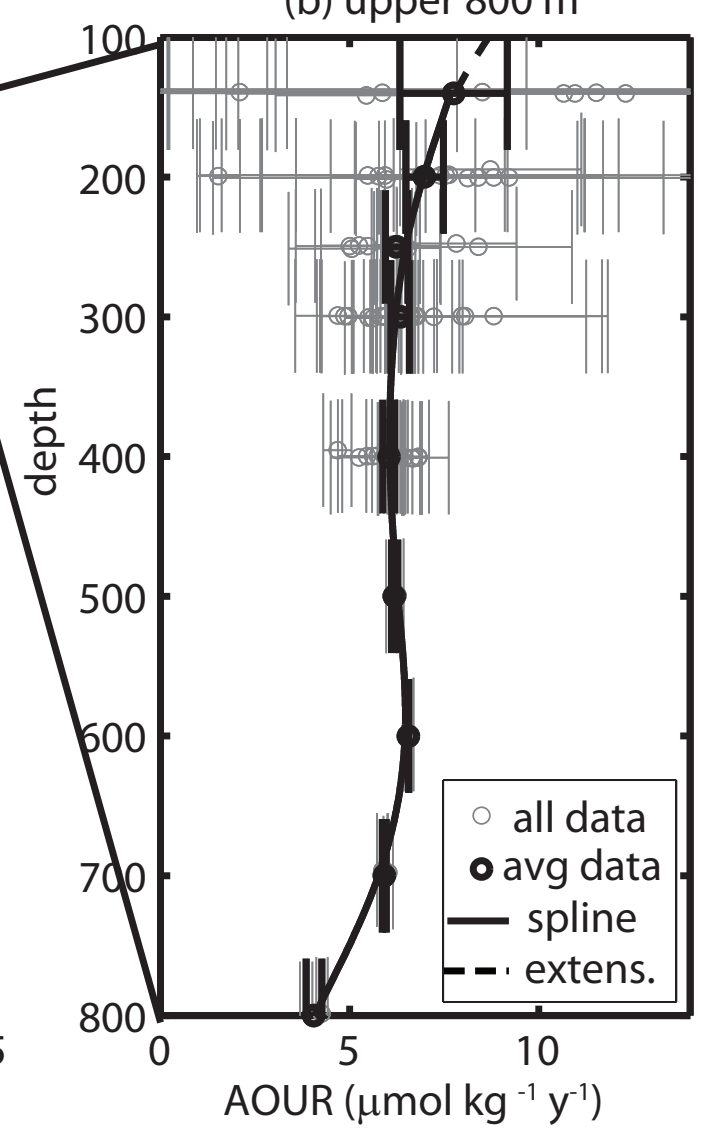


Table 5.3: Estimates of export production in different depth regions of the water column. The prediction for the depth region $200 \mathrm{~m}$ to $800 \mathrm{~m}$ is the most robust prediction. The other regions are shown in order to assess their approximate contribution. The notes explain the limitations of the estimates for the other regions.

\begin{tabular}{ccc}
\hline Depth Range & $\begin{array}{c}\text { Export } \\
\left(\mathrm{mol} \mathrm{O}_{2} \mathrm{~m}^{-2} \mathrm{y}^{-1}\right)\end{array}$ & Notes \\
\hline 75 to $140 \mathrm{~m}$ & 0.57 & $\begin{array}{r}\text { estimated by extending quadratic fit to the } 140 \text { to } 300 \mathrm{~m} \text { data } \\
\text { region is uncomfortably close to } \mathrm{O}_{2} \text { maximum }\end{array}$ \\
$200 \mathrm{~m}$ to $800 \mathrm{~m}$ & 3.73 & calculated from spline fit to AOUR data, most robust region \\
$800 \mathrm{~m}$ to $1000 \mathrm{~m}$ & 0.28 & estimated by extending quadratic fit to the 500 to $800 \mathrm{~m}$ data \\
& total: 5.0 & \\
\hline
\end{tabular}

AOUR estimate of export production is a lower bound. The region between 200 and $800 \mathrm{~m}$ contributes to approximately $75 \%$ of the total export flux.

The AOUR profile determined in this study is surprisingly uniform with depth over the region between 250 and $600 \mathrm{~m}$. Near the surface, AOUR is expected to be higher because organic matter is more abundant and probably more labile. Deeper in the water column, the combination of a smaller amount of probably more refractory organic matter should lead to lower AOUR. Data from sediment traps suggests that remineralization decreases with depth following a power law (Martin et al., 1987). Traditional sediment trap data in the upper ocean, however, have limitations due to artifacts from hydrodynamic biases and swimmers (Gardner, 2000). Nonetheless, recent experiments with neutrally buoyant sediment traps (which avoid many of the problems associated with traditional traps) also show a decrease in remineralization with depth that follows a power law dependence (Buesseler et al., 2007), quite unlike the relatively constant dependence on depth that we see here.

Previous estimates of AOUR in the North Atlantic subtropical gyre show a range of behavior. Some estimates suggest AOUR decreases more steeply with depth than what we found here (Sarmiento et al., 1990) while other estimates show that AOUR below approximately $300 \mathrm{~m}$ is relatively constant (Riley, 1951). Using Radium-228, Sarmiento et al. (1990) calculated an AOUR profile that decreases uniformly 
from about $16 \mu \mathrm{mol} \mathrm{kg}^{-1} \mathrm{y}^{-1}$ near the surface to $0 \mu \mathrm{mol} \mathrm{kg}^{-1} \mathrm{y}^{-1}$ at a depth of $700 \mathrm{~m}-$ both the decrease and the magnitude of Sarmiento et al's AOUR estimates are larger than the ones we calculate here. Using a similar method to the one described here, Jenkins (1980) calculated an AOUR profile that decreases much more sharply between $140 \mathrm{~m}$ and $400 \mathrm{~m}$ than does the profile we present here. However, between 400 and $800 \mathrm{~m}$, the Jenkins (1980) profile and ours have a similar slope. Similarly, the AOUR profile of Riley (1951) drops more sharply between $200 \mathrm{~m}$ and $300 \mathrm{~m}$ than our profile does, but at depths greater than $300 \mathrm{~m}$ both profiles are relatively constant. We have samples from 12 deep profiles over 3 years which we have not yet analyzed for ${ }^{3} \mathrm{He}$. It will be interesting to see if these samples show a relatively uniform remineralization profile with depth or if they show a sharper decrease.

The magnitude of the vertically integrated AOUR here is in the middle of the range of other vertically integrated AOUR estimates in the region. Our value of 3.8 to $5 \mathrm{~mol} \mathrm{O}_{2} \mathrm{~m}^{-2} \mathrm{y}^{-1}$ (depending on the limits of the vertical integration) is larger than estimates by Riley (1951) and smaller than estimates by Jenkins $(1980,1987)$ and Sarmiento et al. (1990). Although our vertically integrated estimate is smaller than that of Jenkins (1980), the difference is driven by the much larger AOUR estimates of Jenkins in the top $300 \mathrm{~m}$. Below $300 \mathrm{~m}$, our AOUR estimates are larger than that of Jenkins because our $\tau$ are smaller and AOU is approximately the same. Jenkins used a similar box model as described here to calculate ventilation ages, but he used tritium or excess ${ }^{3} \mathrm{He}$ to calculate $\tau$ rather than using the T/He age as we did.

Several issues exist regarding the estimation of export production through AOUR calculations. One uncertainty, as described above, is that this calculation is most robust between 200 and $800 \mathrm{~m}$ and thus we are forced to extrapolate to get an estimate of export production above $200 \mathrm{~m}$. From the "extensions" of our AOUR profile, as described above, we found that export production at water depths shallower than $200 \mathrm{~m}$ may be $30 \%$ of the total water column export. Export production above $200 \mathrm{~m}$ may be a significant portion of the total and thus a more robust way of calculating export production in this region would be desirable. Export production below $800 \mathrm{~m}$ probably accounts for less than $10 \%$ of the total export and thus the weakness of our approach at these depths is probably less important.

Other issues arise from the basic premise on which these calculations rate - that the difference between oxygen concentration measured and the saturation oxygen concentration divided by the ventilation time is a valid estimate of the amount of organic matter remineralized. Oxygen at the surface is probably not 
exactly in equilibrium. Even though the water at BATS is not the source water for the deeper water column, $\mathrm{O}_{2}$ measurement at BATS may give us an idea of the deviation from equilibrium. Measurements of $\mathrm{O}_{2}$ in the mixed layer at BATS show that $\mathrm{O}_{2}$ ranges from being undersaturated by approximately $1 \%$ to $2 \%$ in the winter to being supersaturated by $2 \%$ to $3 \%$ in the summer. Stommel (1979) argued that a "demon" selectively pumps late winter water into the main thermocline. Modeling experiments confirmed Stommel's hypothesis (Williams et al., 1995), finding that the subduction period lasts about one month after the end of winter. In the late winter/early spring at BATS, the $\mathrm{O}_{2}$ saturation ranges from $99 \%$ to $101.5 \%$ depending upon the year. If we calculate the integrated AOUR assuming surface water is at $99 \%$ of saturation value, then we determine the integrated AOUR from 200 to $800 \mathrm{~m}$ to be $3.6 \mathrm{~mol} \mathrm{O}_{2} \mathrm{~m}^{2} \mathrm{y}^{-1}$, a decrease in $6 \%$ over our initial calculation. If we calculate the integrated AOUR assuming surface water is at $101.5 \%$ of saturation value, then we determine the integrated AOUR from 200 to $800 \mathrm{~m}$ to be $4.1 \mathrm{~mol} \mathrm{o}_{2} \mathrm{~m}^{2} \mathrm{y}^{-1}$, an increase of $10 \%$. Thus uncertainty in the initial saturation value of $\mathrm{O}_{2}$ leads to an uncertainty of order $10 \%$ in our calculations.

This method uses a total $\mathrm{O}_{2}$ decrease divided by the ventilation age to calculate the export production. But the question arises of when and where exactly was the $\mathrm{O}_{2}$ consumed by remineralization? The $\mathrm{O}_{2}$ probably was not consumed linearly with time. This approach yields no information on whether the $\mathrm{O}_{2}$ was consumed immediately after the water parcel was subducted or if it was consumed the day before we collected the sample. Rather it gives the average AOUR consumed along the path of the water parcel and this may bias the estimates toward shallow conditions.

A final question is the suitability of our box model calculation of ventilation age. In the most basic sense, the concept of "a" ventilation age is flawed since in actuality, there is no single age for the transit time of water between the surface and the interior. Water mixes and thus there is a distribution of ages rather than a single ventilation age. Additionally, different tracers favor different parts of this distribution and hence different tracers yield different ages (Waugh et al., 2003). Nonetheless, by using a box model to calculate tritium, we are taking into account mixing and thus calculating a more accurate age than had we just used the tritium-helium age. Additionally, by using both tritium and helium, we achieve some independence from uncertainties in initial conditions. In the future we will explore a more sophisticated treatment of ventilation age. 
Finally, the box model rests on the assumption that the system is very diffusive. Several lines of evidence support this theory. First, a "pipe" model can be used to estimate that a Peclet number of 1 (i.e. diffusive system) gives the best fit to scatter plots of tritium and ${ }^{3} \mathrm{He}$ (Jenkins, 1988a). Additionally, by using multiple age tracers, Waugh et al. (2003) has shown that the mean age $\Delta$ and the width $\Gamma$ of the transit time distribution are on the same order and thus again that the Peclet number is on order 1 . Thus the use of our box model is appropriate.

\subsubsection{Oxygen and Argon Time-Series}

The data and base case saturation anomaly differences between $\mathrm{O}_{2}$ and Ar are presented in Figure 5-11. The largest differences between the saturation anomalies of $\mathrm{O}_{2}$ and Ar occur in the summer in the region between base of the euphotic zone and base of the mixed layer (Fig. 5-11a). In this region, $\mathrm{O}_{2}$ builds up because of production but is not lost to the atmosphere by gas exchange flux. The model reflects the basic structure of the data's saturation anomaly pattern (Fig. 5-11b), but the $\Delta \mathrm{O}_{2}-\Delta$ Ar maxima in the optimized model are smaller than those in the data. Additionally the maxima persist deeper in the water column for the data than for the model. The negative $\mathrm{O}_{2}$ saturation anomalies between 100 and $200 \mathrm{~m}-\mathrm{a}$ sign of $\mathrm{O}_{2}$ consumption - are stronger in the data than the model, suggesting the model may need more consumption between 100 and $200 \mathrm{~m}$.

The NCP estimates from the inverse modeling range from 3.0 to $5.2 \mathrm{~mol} \mathrm{O}_{2} \mathrm{~m}^{-2} \mathrm{y}^{-1}$, with our base case predicting a value of $3.52 \mathrm{~mol} \mathrm{O}_{2} \mathrm{~m}^{-2}$ (Table 5.4). The model has a difficult time constraining production in the winter time (see discussion below) and thus we also report the "summer" production - production during the six month period between Apr 1 and Sept 30. For this period, the production estimates range from 1.96 to $2.41 \mathrm{~mol} \mathrm{O}_{2}$, with the base case predicting a value of $2.13 \mathrm{~mol} \mathrm{O}_{2} \mathrm{~m}^{-2}$. This summer prediction is much more tightly constrained than the annual production.

The magnitude of the consumption magnitude $C$ estimated for the model ranges from 6 to $11 \mathrm{~mol} \mathrm{O}_{2}$ $\mathrm{m}^{-2} \mathrm{y}^{-1}$ integrated over the $1000 \mathrm{~m}$ water column included in the model, considerably larger than the AOUR estimates of consumption. This consumption is also much larger than the production. However, a model that is using data in the upper $160 \mathrm{~m}$ cannot constrain consumption over $1000 \mathrm{~m}$ of water column. Instead, the model is only constraining consumption in the upper 200 to $300 \mathrm{~m}$ since it is only consumption there 
Figure 5-11: The difference in saturation anomaly of $\mathrm{O}_{2}$ and $\mathrm{Ar}$ is a measure of net community production. Contour plots of $\Delta \mathrm{O}_{2}-\Delta \mathrm{Ar}$ for both the (a) data and (b) model show maxima in the summertime between the base of the mixed layer and the base of the euphotic zone. The thin white line demarcates the mixed layer and the circles mark locations of samples. Contour lines are drawn at $2 \%$ intervals.
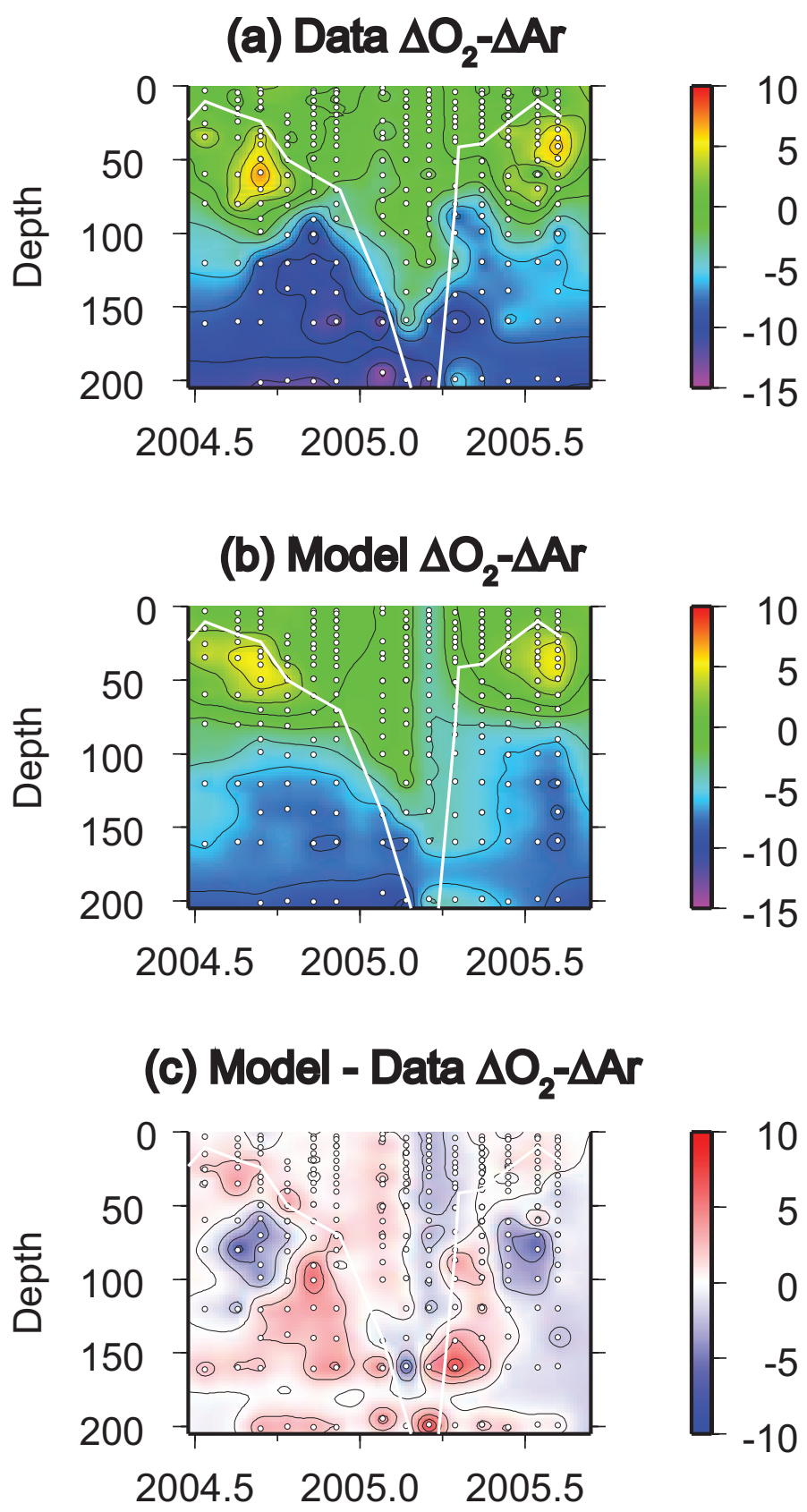
Table 5.4: Net community production as estimated from the inverse fit to the euphotic zone seasonal cycles of $\mathrm{O}_{2}$ and Ar. The annual production is listed as well as the production between Apr 1 and Sept. 30. The model constrains this "summer" production more tightly than the full annual cycle. The base case uses a compensation depth of $75 \mathrm{~m}$, includes samples with depths up to $160 \mathrm{~m}$ in the cost function, and uses a seasonal modulation of production with amplitude of 1 . We examined the sensitivity of our results to other choices, as listed here, with the notes describing the conditions of each run.

\begin{tabular}{cccl}
\hline$\#$ & $\begin{array}{c}\text { Annual Prod } \\
\left(\mathrm{mol} \mathrm{O}_{2} \mathrm{~m}^{2} \mathrm{y}^{-1}\right)\end{array}$ & $\begin{array}{c}\text { Apr to Sept } \\
\text { Prod }\left(\mathrm{mol} \mathrm{O}_{2}\right)\end{array}$ & Notes \\
\hline
\end{tabular}

Base Case
1.
3.52
2.13

\section{Different Physical Parameters}

$\begin{array}{llll}\text { 2. } & 3.23 & 1.96 & \mathrm{Kz}=4 \times 10^{-5} \mathrm{~m}^{2} \mathrm{~s}^{-1}, E k m=16 \mathrm{~W} \mathrm{~m} \mathrm{~W}^{-2} \\ \text { 3. } & 3.75 & 2.27 & \mathrm{Kz}=6 \times 10^{-5} \mathrm{~m}^{2} \mathrm{~s}^{-1}, \mathrm{Ekm}=20 \mathrm{~W} \mathrm{~m}^{-2}\end{array}$

Different Gas Exchange Parameters
4.
3.52
2.14
5.
3.51
2.13

Different Depths Included in Cost Function

$\begin{array}{lll}6 . & 3.02 & 1.83 \\ 7 . & 3.24 & 1.96 \\ 8 . & 3.37 & 2.05 \\ 9 . & 3.66 & 2.22\end{array}$

Different Representation of Consumption
10.
3.09
1.88
$11 . \quad 3.53$
2.14
12.
3.30
2.00
compensation depth $=100 \mathrm{~m}$ set consumption below $300 \mathrm{~m}$ to zero force consumption in upper $300 \mathrm{~m}$ to equal production

Different Seasonal Modulation

$\begin{array}{lll}13 . & 5.23 & 2.20 \\ 14 . & 4.01 & 2.07 \\ 15 . & 3.49 & 2.29 \\ 16 . & 3.91 & 2.27 \\ 17 . & 4.36 & 2.41\end{array}$

depth to which points included in cost function $=100 \mathrm{~m}$ depth to which points included in cost function $=120 \mathrm{~m}$ depth to which points included in cost function $=140 \mathrm{~m}$ depth to which points included in cost function $=200 \mathrm{~m}$

to $=0$ : production peaks $\sim$ two months earlier to $=0.1:$ production peaks $\sim$ one month earlier to $=0.3$ : production peak $\sim$ one month later seasonal amplitude of production $a=0.75$ seasonal amplitude of production $a=0.5$ 
that affects the $\mathrm{O}_{2}$ signal that is being used in the cost function. The integrated consumption in the upper $300 \mathrm{~m}$ of the model is $3.3 \mathrm{~mol} \mathrm{O}_{2} \mathrm{~m}^{-2} \mathrm{y}^{-1}$ for the base case, roughly in balance with the $\mathrm{O}_{2}$ production. We examined the sensitivity of our results to how we modeled consumption. The NCP estimates did not change significantly when we either set the consumption below $300 \mathrm{~m}$ to equal zero or when we forced consumption in the upper $300 \mathrm{~m}$ to exactly balance the production.

We examined the sensitivity of the results to the choice of physical parameters and to the choice of gas exchange parameters, in both cases using sets of parameters that were found to be appropriate in Chapter 4 of this thesis. Production increases if the vertical diffusivity increases. With a larger $K_{z}$, there is a larger flux of low oxygenated water into the euphotic zone that has the effect of "canceling" out the $\mathrm{O}_{2}$ produced by biological production. Thus more production is needed to maintain the same $\Delta \mathrm{O}_{2}-\Delta$ Ar signal. The results are not sensitive to the choice of gas exchange parameters used. Since we are looking at the difference in saturation anomalies between $\mathrm{Ar}$ and $\mathrm{O}_{2}$, the effects of gas exchange are minimized.

The production estimates vary by up to $15 \%$ if different depths are used as the cut-off depth for the cost function. When a shallower depth is used, the production decreases. In the late fall/early winter mixed layer, the model predicts $\Delta \mathrm{O}_{2}-\Delta \mathrm{Ar}$ to be larger than the data (Figure 5-12). When the cut-off depth decreases, these mixed layer points are relatively more important and thus the total production decreases in order to achieve a better match to the data.

The production estimates are sensitive to the choice of seasonal modulation of production. The dependence of NCP on season is not well known. Seasonal cycles of DIC and $\delta^{13} \mathrm{C}$ have been used to infer that although primary production peaks in the bloom season in early spring, NCP may peak later (Gruber et al., 1998). The relative proportion between summer and winter NCP is not known. In the winter, deeper mixed layers increase availability of nutrients but limit light. We thus examined the sensitivity of our results to the seasonal modulation. We examined the effect of a temporal shift (by changing to) and the effect of shallowing the gradient between summer and winter production (by changing $a$ ). When the peak in production is shifted earlier by one month, the annual estimated production increases by $15 \%$. If it is shifted earlier by two months, then the annual estimated production increases by $40 \%$ but the amount of production between April and September increases by only $3 \%$. If the peak is shifted later by one month, then the annual estimated production estimate changes by only $1 \%$. 
Figure 5-12: The difference in saturation anomalies of $\mathrm{O}_{2}$ and $\mathrm{Ar}$ in the mixed layer. The model overestimates $\Delta \mathrm{O}_{2}-\Delta \mathrm{Ar}$ in the late fall/early winter and then underestimates $\Delta \mathrm{O}_{2}-\Delta \mathrm{Ar}$ when the mixed layer depth sharply increases in February 2005. The deep mixed layer brings up water that is undersaturated in $\mathrm{O}_{2}$.

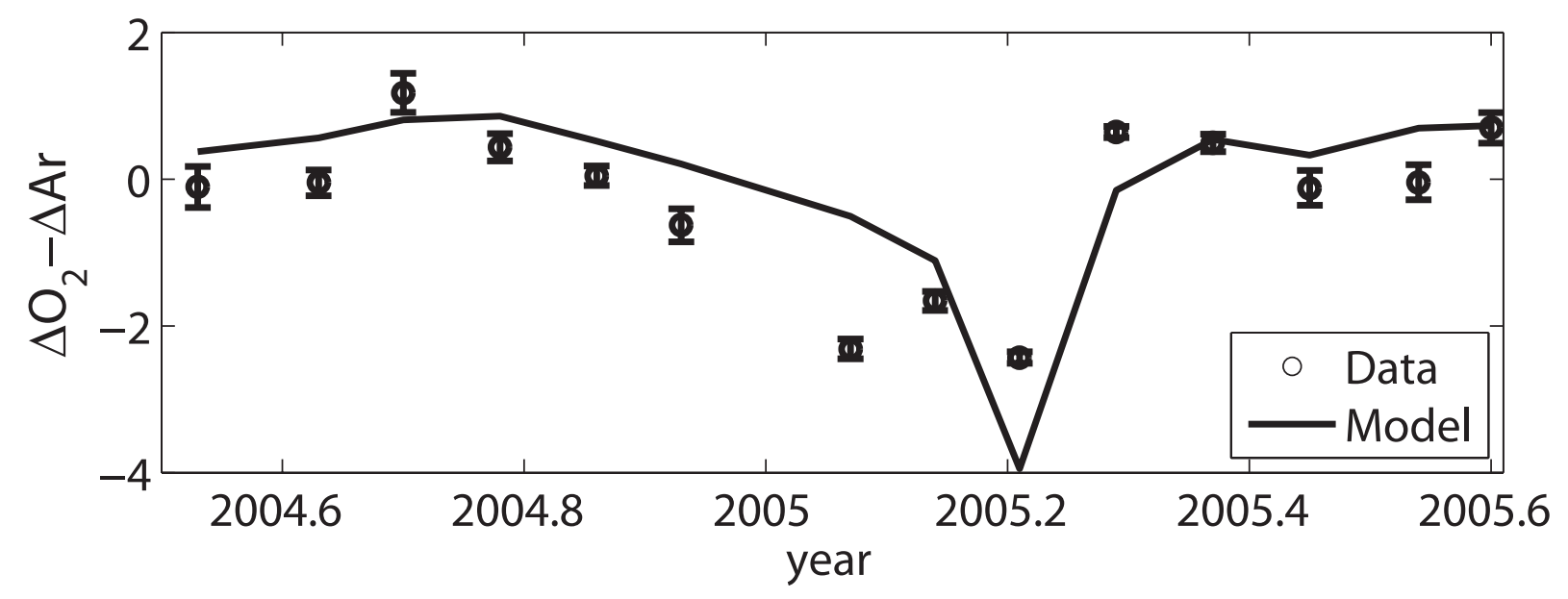

The results are also sensitive to the amplitude of seasonal production. If the seasonal amplitude of production is decreased to 0.5 , then there is a larger fraction of production in the winter (see Figure 53 ), and the annual production increases by almost $30 \%$. However, the "summer" production (production between Apr 1 and Sept 30) changes by only 13\%, suggesting the model is better at constraining summer production than annual production. In the winter, there is a larger gas exchange flux and a deep mixed layer depth (deeper than the maximum in production). Thus $\mathrm{O}_{2}$ due to winter production is rapidly outgassed and mixed down into the consumption zone. The error structure (deviation between $\Delta \mathrm{O}_{2}-\Delta \mathrm{Ar}$ model and data) is remarkably similar for the model runs with different seasonal amplitudes. When the seasonal amplitude decreases, the optimal model runs have a similar amount of vertically integrated production in the summer but a larger amount in the winter (Figure 5-13a). The $\Delta \mathrm{O}_{2}-\Delta \mathrm{Ar}$ model values at $35 \mathrm{~m}$ are remarkably similar in spite of the difference in winter production (Figure 5-13b). Thus our method does not constrain winter production well.

In summary, this technique relies on (1) $\Delta \mathrm{O}_{2}-\Delta$ Ar being present (not "washed" away by deep mixing or gas exchange) and (2) $\Delta \mathrm{O}_{2}-\Delta$ Ar being a measure of production. As shown above, in the winter, (1) fails due to deep mixed layers and increased gas exchange. We use a model in order to characterize any verti- 
Figure 5-13: A sensitivity study of the model's response to the amplitude of the seasonal modulation of production. (a) A steeper amplitude, $a$, leads to a larger difference between summer and winter production. (b) However, very little difference is seen in the difference in saturation anomalies between $\mathrm{O}_{2}$ and Ar, even at $35 \mathrm{~m}$ where this signal is strongest. This may be because the largest difference in production occurs in late fall/winter when (c) the total gas exchange flux is large and (d) the mixed layer is deeper than $35 \mathrm{~m}$. The dashed line in $(d)$ indicates a mixed layer depth of $35 \mathrm{~m}$.
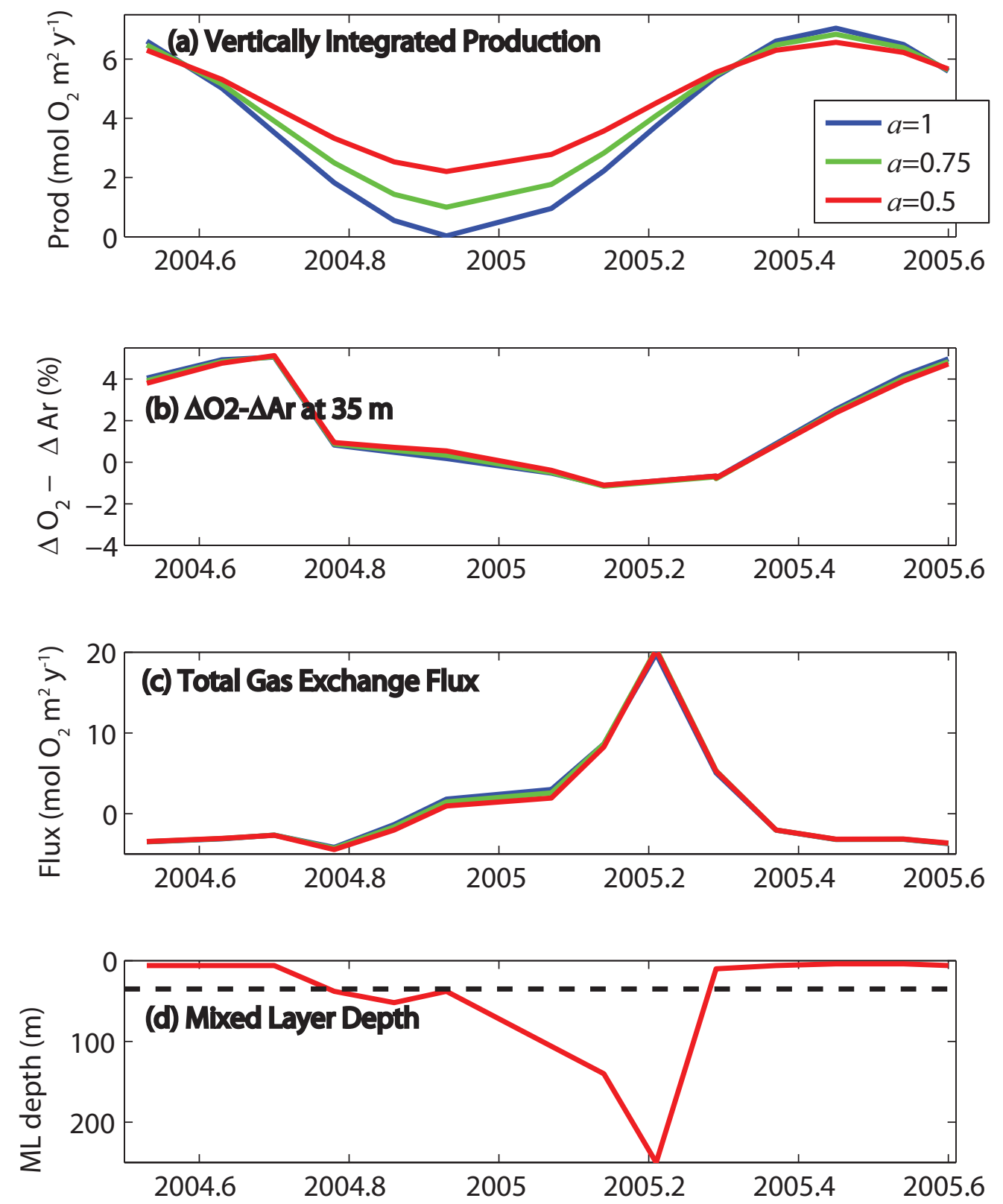
cal transport of low oxygenated waters which would confound (2). However, uncertainties in the model's dynamics (such as the turbulent diffusivity) will lead to uncertainty in the NCP estimate. Additionally, our model ignores lateral transport, which may be different for $\mathrm{O}_{2}$ and for Ar.

Future work should include examining the sensitivity of NCP estimates to the vertical shape of the production profile. In this study, production was assumed to be inhibited near the surface. However, the vertical profile of NCP is not well known and thus production may not be decreasing in the mixed layer as sharply as we assumed. If a profile of NCP was used that was uniform with respect to depth, then the estimate of NCP would probably increase.

The estimate of NCP determined here (3.0 to $5.2 \mathrm{~mol} \mathrm{O}_{2} \mathrm{~m}^{-2} \mathrm{y}^{-1}$ ) is within the range of NCP previously estimated for the Sargasso Sea using seasonal $\mathrm{O}_{2}$ cycles. Using an $\mathrm{O}_{2}$ record from the 1960 to 1980, Jenkins and Goldman (1985) estimated production to be approximately $5 \mathrm{O}_{2} \mathrm{~m}^{-2} \mathrm{y}^{-1}$. Musgrave et al. (1988) reexamined the same $\mathrm{O}_{2}$ data from 1961 to 1970 using a one-dimensional upper ocean model and found a production of 3 to $4 \mathrm{~mol} \mathrm{O}_{2} \mathrm{~m}^{-2} \mathrm{y}^{-1}$. Spitzer and Jenkins (1989) used a time-series of $\mathrm{Ar}$ and $\mathrm{O}_{2}$ for the year 1985 and estimated a production of $4.3 \pm 1.7 \mathrm{O}_{2} \mathrm{~m}^{-2} \mathrm{y}^{-1}$.

In this study, we use the same model as Spitzer and Jenkins with the following differences: (1) Spitzer and Jenkins used climatological winds and heat fluxes whereas we use QuikSCAT winds and NCEP reanalysis heat fluxes; (2) Spitzer and Jenkins used a three-step linear parameterization of equilibrium gas exchange (Liss and Merlivat, 1986), and a formulation of air injection where partial bubble trapping depended only on the diffusivity of the gas whereas we use a quadratic gas formulation of gas exchange and the gas exchange parameterizations determined in Chapter 4 of this thesis; (3) Spitzer and Jenkins forced production to balance consumption at every time step whereas we solve independently for production and consumption and (4) Spitzer and Jenkins used different vertical profiles of production (for example a compensation depth of $100 \mathrm{~m}$ and an exponentially decreasing consumption) and a seasonal modulation that peaked in spring; (5) Spitzer and Jenkins used linear inversion (SVD) whereas we use a non linear optimization scheme. Our sensitivity studies, however, show that these differences should not significantly change the estimate of production and thus the similarity between our estimates and those of Spitzer and Jenkins suggest there may not be a significant long-term change (of more than $1 \mathrm{~mol} \mathrm{O}_{2} \mathrm{~m}^{-2} \mathrm{y}^{-1}$ ) in NCP in the Sargasso Sea over the past twenty years. 


\subsection{Synthesis}

Over sufficiently long temporal and spatial scales, new production, export production, and net community production should balance (Eppley and Peterson, 1979). It is instructive to examine the relationship between the three types of production as estimated in this study (Table 5.2). To do so, however, we must understand the temporal and spatial scales of the methods used here. Although the new production estimate from the helium flux gauge is based on one year of helium fluxes, the actual time-scale of new production it applies to is much longer, on the order of years, and it applies to a regional spatial scale. The AOUR export production calculation integrates over years to decades and again is regional in spatial scale. We do not know exactly when or at what depth the export took place but only that oxygen was consumed sometime between when the water left the surface and when the water was "dated" years to decades later in the thermocline. In contrast, the estimate of $\mathrm{NCP}$ is a local estimate that only reflects production during one year, and is most accurate for a period of six months when the mixed layer is shallow and the gas exchange flux is small.

The NCP agrees remarkably well with the export production estimate, in spite of the difference in temporal and spatial scales of the two methods. In contrast, the new production estimate from the ${ }^{3} \mathrm{He}$ flux gauge is twice as large as the other estimates. It is hard to believe that new production is actually double export production and NCP. If so much new production were occurring, then there should be some sign of it - both immediately (in NCP) and afterward (in export production). The new production estimate was calculated for nitrate and then the revised Redfield ratios of Anderson and Sarmiento (1994) were used to convert it into $\mathrm{O}_{2}$ "currency" for comparison with the other methods. Thus any uncertainties in this ratio could lead to disagreement between methods. However, the expected sense of this uncertainty works in the opposite direction; at BATS, nitrate may be preferentially remineralized (Ono et al., 2001; Anderson and Pondaven, 2003) and thus exported matter has a high $\mathrm{C} / \mathrm{N}$ ratio. $\mathrm{C}$ should be inextricably linked with $\mathrm{O}$ since most of the oxygen is consumed when converting organic matter to $\mathrm{CO}_{2}$ and thus a high $\mathrm{C} / \mathrm{N}$ ratio results in an even larger discrepancy between the ${ }^{3} \mathrm{He}$ flux gauge estimate of new production and the estimates of export production and NCP.

A large uncertainty in the ${ }^{3} \mathrm{He}$ flux gauge calculation stems from the regression slope of ${ }^{3} \mathrm{He}$ and $\mathrm{NO}_{3}^{-}$. As mentioned above, this slope is changing with time and thus the ${ }^{3} \mathrm{He}$ flux gauge may be overestimating production. If the slope from a decade ago is used instead of today's slope, then the ${ }^{3} \mathrm{He}$ flux gauge new 
production estimate would become statistically equivalent to the export and NCP estimates from this study. The flux gauge may overestimate production for a second reason - it is possible that the water that is transported into the euphotic zone at BATS has already circled round the gyre and has been "trapped" between the mixed layer and base of the euphotic zone. Therefore, there may have been upstream removal of $\mathrm{NO}_{3}^{-}$without complete loss of ${ }^{3} \mathrm{He}$ in the more productive regions to the north. Thus, the ${ }^{3} \mathrm{He}$ flux gauge is a regional-scale estimate of new production, rather than a local one.

The new production estimate from this study is statistically equivalent to that calculated by Jenkins and Doney (2003) and by Jenkins (1988b), although it is on the high side of both estimates (Table 5.5). Those estimates were on the high side, but again statistically equivalent, to estimates of export production and NCP made through a variety of geochemical tracer techniques. The export production and NCP measured here are within the range, albeit slightly on the low side, of other estimates of export production and NCP measured in the Sargasso Sea using geochemical tracers. At first glance, such agreement between different types of production and different methods is heartening. On closer examination, however, it reveals a paradox.

The ${ }^{3} \mathrm{He}$ flux gauge technique describes the transport of water from the upper thermocline to the euphotic zone. This water brings ${ }^{3} \mathrm{He}$, nitrate, and phosphate as described above but also must bring an $\mathrm{O}_{2}$ debt and a DIC surplus, since the remineralization processes that create the nitrate and phosphate, also create DIC and consume $\mathrm{O}_{2}$. If the remineralization quotient approximately equals the photosynthetic quotient, as is believed (Laws, 1991), then if new production and NCP were equal, the $\mathrm{O}_{2}$ debt carried by the water should equal the $\mathrm{O}_{2}$ produced in the euphotic zone and thus no $\mathrm{O}_{2}$ maximum would be apparent. Similarly, the surplus of DIC would "cancel" the DIC consumed in the euphotic zone, and no euphotic zone DIC minimum would be measured. In short, the current understanding of remineralization and physical transport of nutrients implies that there should not be any euphotic zone $\mathrm{O}_{2}$ maximum, and yet such an oxygen maximum is observed. In fact, if new production is really larger than NCP as this study implies, then the problem is even worse - the new production should bring up such a deficit of $\mathrm{O}_{2}$ as to not only completely obliterate the euphotic zone oxygen cycles but as to even turn them negative. However, there are significant difference in C:N ratio (Ono et al., 2001) at BATS and thus perhaps the euphotic zone $\mathrm{O}_{2}$ signal stems from the excess $\mathrm{O}_{2}$ caused by export of high $\mathrm{C}: \mathrm{N}$ material in extreme oligotrophy. If the water being transported into the mixed layer has an $\mathrm{O}_{2}$ debt associated with typical C:N ratios (because it comes from elsewhere 
Table 5.5: Estimates of biological production from past research in the Sargasso Sea. Fluxes have all been converted into $\mathrm{O}_{2}$ currency using the revised Redfield ratios of Anderson and Sarmiento (1994).

\begin{tabular}{cccc}
\hline Type of Production & Method & $\begin{array}{c}\text { Flux } \\
\left(\mathrm{O}_{2} \mathrm{~mol} \mathrm{~m}^{2} \mathrm{y}^{-1}\right)\end{array}$ & Reference \\
\hline \multirow{2}{*}{ New Production } & He Flux Gauge & $9.6 \pm 3$ & this study \\
& He Flux Gauge & $6.0 \pm 1.7$ & $\begin{array}{c}\text { Jenkins (1988b) } \\
\text { He Flux Gauge }\end{array}$ \\
& $8.9 \pm 2.3$ & Jenkins and Doney (2003) \\
Export Production & AOUR: Thermocline invasion & 3.7 to 5.0 & this study \\
& AOUR: Thermocline invasion & $5.1 \pm 1$ & Jenkins (1980) \\
& Seasonal AOUR amplitude & $4.5 \pm 1$ & Jenkins and Goldman (1985) \\
Net Community Production & & & \\
& O2 and Ar time-series & 3.0 to 5.2 & this study \\
& Oxygen and Ar time-series & $5.4 \pm 1.5$ & Spitzer and Jenkins (1989) \\
& DIC time series & $5.3 \pm 1.4$ & Michaels et al. (1994) \\
& ${ }^{13} \mathrm{C}$ time series (mixed layer estimate only) & $4.6 \pm 1.5$ & Gruber et al. (1998) \\
\hline
\end{tabular}


in the gyre) but biological production produces high $\mathrm{C}: \mathrm{N}$ organic matter, than an excess of $\mathrm{O}_{2}$ would be observed.

One must remember, however, the temporal differences between the processes. The ${ }^{3} \mathrm{He}$ flux gauge showed the largest fluxes in the wintertime when the mixed layer deepens and mines the thermocline. In the winter, both model and data show negative $\mathrm{O}_{2}$ saturation anomalies as the $\mathrm{O}_{2}$ debt is brought up as well. There may be production that is partially compensating for some of this $\mathrm{O}_{2}$ debt. In the late winter/early spring, $\mathrm{O}_{2}$ in the model is close to saturation levels. This may be because $\mathrm{O}_{2}$ produced from new production is balancing the $\mathrm{O}_{2}$ debt brought up from below. This winter production may not be seen by our other techniques. We have shown that the euphotic zone $\mathrm{O}_{2}$ cycles do not constrain the production in the winter. It is harder to explain why the AOUR estimates might be missing this potentially large production signal. If the initial $\mathrm{O}_{2}$ is actually undersaturated, then the AOUR method could also be underestimating the winter production, but calculations done above indicate this should be a small effect. The AOUR estimate does not constrain remineralization near the surface well, and thus may not capture any remineralization that is occurring just below the winter mixed layer. Thus it is possible that there may be large productivity in the winter that is not being caught by the other techniques and that could partially explain some of the "paradox".

Other possibilities for resolving the paradox fail on close examination. For example, one might argue that the paradox could be resolved if the upwelled water lost its oxygen debt via air-sea gas exchange before nitrate was used. If that were the case, then the nutrients could fuel new production and yet the oxygen maxima would not be erased. However, the time scales are in exactly the wrong direction - air-sea gas exchange for oxygen takes several weeks whereas nutrients can be utilized in the oligotrophic ocean in days. Alternately, one could contend that the paradox could be resolved if the photosynthetic quotient was different from the remineralization quotient by more than a factor of two. However, such a difference over long time scales causes mass balance problems (Laws, 1991). Another explanation would be that nitrogen fixation accounts for the same amount of new production as is fueled by upwelled nutrients $(0.5$ to $1 \mathrm{~mol} \mathrm{~N}$ $\left.\mathrm{m}^{-2} \mathrm{y}^{-1}\right)$. However, current estimates of nitrogen fixation in the Sargasso sea are much lower, ranging from so low as to be nondetectable in $\mathrm{N}$ isotopic studies (Knapp et al., 2005) to $0.015 \mathrm{~mol} \mathrm{~N} \mathrm{~m}^{-2} \mathrm{y}^{-1}$ (Orcutt et al., 2001) to $0.072 \mathrm{~mol} \mathrm{~N} \mathrm{~m}^{-2} \mathrm{y}^{-1}$ (Gruber and Sarmiento, 1997). 


\subsection{Conclusions}

We have used geochemical tracer techniques to estimate the amount of new production, export production, and NCP from a fourteen month time series of ${ }^{3} \mathrm{He}, \mathrm{O}_{2}$, Ar, and tritium collected at the BATS site between July 2004 and August 2005. New production is estimated to be $0.9 \pm 0.3 \mathrm{~mol} \mathrm{~N} \mathrm{~m}^{-2} \mathrm{y}^{-1}$ or $9.6 \pm 3 \mathrm{~mol} \mathrm{O}_{2}$ $\mathrm{m}^{-2} \mathrm{y}^{-1}$. Export production and NCP are significantly smaller, with the former estimated to be 3.7 to 5.0 mol $\mathrm{O}_{2} \mathrm{~m}^{-2} \mathrm{y}^{-1}$ and the latter to be 3.0 to $5.2 \mathrm{~mol} \mathrm{O}_{2} \mathrm{~m}^{-2} \mathrm{y}^{-1}$. The export production and NCP production estimates agree well with past estimates of production from geochemical tracers from the Sargasso Sea. The new production estimate is on the high side but statistically equivalent to an earlier estimate by Jenkins and Doney (2003), which is also based on the ${ }^{3} \mathrm{He}$ flux gauge technique.

The differences in new, net community, and export production may be a result of fundamental processes in the ocean or may be a result of uncertainties with our techniques. The largest source of uncertainty in the new production estimate is from the correlation between $\delta^{3} \mathrm{He}_{e x}$ and nitrate. The largest source of uncertainty in the export production estimate is the oxygen deficit resulting from remineralization occurring above $200 \mathrm{~m}$. The water above $200 \mathrm{~m}$ is uncomfortably close to the $\mathrm{O}_{2}$ compensation depth and thus any production occurring there is not easily assessed. Additionally the concept of ventilation ages from our box model is difficult to apply to this young water. The largest source of uncertainty in the NCP estimate is from sizable NCP which could be occurring in the winter but not being recorded in $\Delta \mathrm{O}_{2}-\Delta$ Ar due to deep mixed layers and large gas exchange fluxes.

This study is unique in that it measures three types of production all at the same location at the same time. Samples from another two years of the time-series will be analyzed and will allow us to see if the differences between production estimates persist or if they are due to interannual variability. Eddies for example can have a dramatic effect on biological production (McGillicuddy et al., 2007). Additionally, more samples will allow us to see whether AOUR remains surprisingly constant with depth.

Future work on the ${ }^{3} \mathrm{He}$ flux gauge technique should include relating the $\delta^{3} \mathrm{He}_{e x}$ to phosphate rather than nitrate. The Sargasso sea may be phosphate limited (Wu et al., 2000) and thus using phosphate could result in a better estimate of new production. For the AOUR studies, in the future we could use a more sophisticated treatment of $\tau$. The concept of tracer distribution age (Waugh et al., 2003) could be applied to the estimated $\tau$ in order to calculate a distribution of ventilation ages and thus a distribution of AOUR 
estimates. Additionally, a two-dimensional mixing/advection model could lead to more realistic estimates of ventilation age. Future work should also attempt to use the $\Delta \mathrm{O}_{2}-\Delta \mathrm{Ar}$ seasonal cycles in the upper ocean to constrain export between $200 \mathrm{~m}$ and the surface, blending the seasonal $\mathrm{O}_{2}$-Ar cycles and the AOUR techniques. For all three tracer subsystems, future work should continue to examine the assumptions behind the approaches and should attempt to reconcile the differences in their conclusions. 


\subsection{Acknowledgments}

This work was supported by the National Science Foundation Chemical Oceanography program (OCE0221247) and by the Department of Defense (graduate fellowship to RHRS). We thank the captain and crew of the $R / V$ Weatherbird II, the $R / V$ Cape Hatteras, and the $R / V$ Oceanus for their assistance in collecting samples. We are very grateful to Megan Roadman, Debra Lomas, and other BATS personnel for collecting and running the oxygen and nutrient samples. We thank Mike Lomas, Rod Johnson, and all other BATS personnel for the use of lab-space, ship-time, and assistance. Special thanks go to Carolyn Walker, Dempsey Lott, and Kevin Cahill for collecting samples on three of the cruises and to DeDe Toole and Naomi Levine for their donation of computing time. 
Chapter 6

Conclusions and Future Directions 
Noble gases are ideal oceanographic tracers because they are chemically and biologically inert. Additionally, since the five noble gases have different solubilities and diffusivities, a time-series of all five stable noble gases, as presented in this thesis, can be used to separate and quantify physical and biological processes in the ocean. Time-series of He, Ne, and Ar (Spitzer and Jenkins, 1989) and of Ne, Ar, and $\mathrm{N}_{2}$ (Hamme and Emerson, 2006) have been measured before. This thesis presents the first time-series of $\mathrm{Kr}$ and $\mathrm{Xe}$ in the ocean. These heavier gases are particularly useful because of their strong solubility dependence on temperature, providing a useful contrast to the lighter gases. Using the time-series of all five noble gases, we developed a parameterization of air-sea gas exchange that constrains diffusive gas exchange to $\pm 6 \%$ and air injection to $\pm 15 \%$ for wind speeds between 0 and $13 \mathrm{~m} \mathrm{~s}^{-1}$. Additionally, by combining the time-series of noble gases with helium isotopes, tritium, $\mathrm{O}_{2}$ and $\mathrm{NO}_{3}^{-}$measurements, we quantified new, net community, and export production and examined the relationships between these three types of production.

The first challenge of this thesis was developing a method for precisely measuring the noble gases in seawater samples, particularly for the heavier noble gases which have rarely been measured. We built a processing line which uses automated cryogenic trapping and two mass spectrometers in order to separate and analyze the noble gases. First, water vapor, methane, and active gases are removed from the samples by a dual stage cryogenic trap, a Pd catalyst, and two Ti-Zr-Fe alloy getters. Second, a stainless steel cryogenic trap sorbs and then sequentially releases $\mathrm{Ne}, \mathrm{Ar}, \mathrm{Kr}$ and $\mathrm{Xe}$ while an activated charcoal cryogenic trap sorbs and then releases He. Helium isotopes are measured on a purposely constructed branch-tube magnetic sector mass spectrometer equipped with a secondary electron multiplier. Neon, argon, krypton, and xenon are measured statically on a Hiden quadrupole mass spectrometer with a pulse-counting secondary electron multiplier. Although in principle separating the noble gases by releasing the gases at different temperatures from the cryogenic traps should be straightforward, in practice the cryotraps need to warmed and cooled many times throughout the analysis in order to completely separate the gases. Furthermore, the presence of $\mathrm{Ar}, \mathrm{Xe}$ and $\mathrm{CH}_{4}$ quantitatively influences the trapping and release of $\mathrm{Kr}$. These matrix effects needed to be minimized and assessed. A more inert surface on the stainless steel cryogenic trap might reduce some of these problems. The reproducibility of our method for gas standards is better than $0.1 \%$ for He and Ar, better than $0.15 \%$ for $\mathrm{Ne}$ and $\mathrm{Kr}$ and better than $0.2 \%$ for Xe. For water samples, the reproducibility is worse, probably reflecting variability introduced during sample collection and initial extraction of the gases. The 
most likely source of this variability is from bubbles entrained during sample collection. The reproducibility for water samples as assessed from duplicate pairs of samples is $\sim 1 \%$ for $\mathrm{He}$ and $\mathrm{Ne}, \sim 0.3 \%$ for $\mathrm{Ar}$ and $\mathrm{Kr}$, and $\sim 0.2 \%$ for Xe. Xenon is the gas which is least affected by bubbles and it is only gas where the reproducibility of samples is equal to the reproducibility of gas standards.

In order to quantitatively use the noble gas measurements, we employ a one-dimensional model to capture the physical dynamics of the water column at the Bermuda Atlantic Time-series Study (BATS) site. We thus extended a modified Price-Weller-Pinker model (Price et al., 1986; Spitzer and Jenkins, 1989) to the heavier noble gases and made the model more "realistic" by including NCEP reanalysis forcing and QuikSCAT winds. We performed a sensitivity study to see how well the noble gases could constrain air-sea gas exchange parameters. Using linear inverse techniques (singular value decomposition) we determine that a time-series of all five noble gases at BATS could constrain diffusive air-sea gas exchange to order $10 \%$ and air injection to $14 \%$. However, this sensitivity study assumes smaller measurement uncertainty than we were actually able to achieve, assuming a $0.1 \%$ measurement uncertainty for $\mathrm{He}$ and $\mathrm{Ne}$.

A fourteen month time-series of all five noble gases measured between July 2004 and August 2005 at 22 depths in the upper $400 \mathrm{~m}$ of the Sargasso Sea each month is presented as part of this thesis. As expected, the saturation anomalies of the noble gases follow seasonal patterns, with the the saturation anomalies of the heavier gases being at a maximum in the seasonal thermocline in the summer. In the summer, the water warms, the heavier gases are less soluble at warmer temperatures, resulting in a supersaturation and a flux of the gas out of the water column. More surprisingly, the heavier gases are also slightly supersaturated in the winter. This may reflect errors in the solubility functions of the noble gases or could be a result of air injection.

We use a non linear, constrained optimization technique to calculate air-sea gas exchange parameters from the noble gas time-series. Based on error analysis, uncertainties in the observations, uncertainties in the solubility functions, and the range of physical parameterizations consistent with the model and data, the diffusive gas exchange rate was constrained to $\pm 6 \%$ and the air injection rate to $\pm 15 \%$. We determine the diffusive gas exchange rate to be $20 \%$ smaller than the most commonly used relationship proposed by Wanninkhof (1992). Additionally, we find that air injection is more important than previously has been thought, even for the more soluble gases such as $\mathrm{Kr}$ and Xe. Most air-sea gas exchange parameterizations 
do not include air injection explicitly and thus they may be missing a significant fraction of the air-sea gas exchange flux. The parameterizations developed here can be used with any gas in other oligotrophic regions of the ocean with moderate wind speeds $\left(0<u_{10}<13 \mathrm{~m} \mathrm{~s}^{-1}\right)$. The parameterizations may not be applicable in highly productive regions, since surfactants could lead to a dampening of the diffusive gas exchange, or in regions with high wind speeds $\left(\mathrm{u}_{10}>15 \mathrm{~m} \mathrm{~s}^{-1}\right)$ outside the range encountered in this study. Future work should include combining the data set collected here with satellite altimetry and backscatter measurements in order to calibrate air-sea gas exchange flux parameterized as a function of surface roughness as well as wind speed (Woolf, 2005; Glover et al., 2007; Frew et al., submitted, 2007).

Biological production was estimated from the same time-series of noble gases, as well as from helium isotopes, tritium, $\mathrm{O}_{2}$, and $\mathrm{NO}_{3}^{-}$measurements. The improved gas exchange parameters calculated in this thesis were used in order to calculate the shift in equilibrium value of $\delta^{3} \mathrm{He}$ due to air injection and to model the diffusive gas exchange and air injection fluxes of $\mathrm{O}_{2}$ from the euphotic zone. The ${ }^{3} \mathrm{He}$ flux gauge technique (Jenkins, 1988b; Jenkins and Doney, 2003) was used to calculate that new production at BATS is equal to $0.90 \pm 0.3 \mathrm{~mol} \mathrm{~N} \mathrm{~m}^{-2} \mathrm{y}^{-1}$, which is equivalent to $9.6 \pm 3 \mathrm{~mol} \mathrm{O}_{2} \mathrm{~m}^{-2} \mathrm{y}^{-1}$. This result is slightly higher than, but consistent with, earlier results. Export production was calculated from apparent oxygen utilization rates to be 3.7 to $5.0 \mathrm{~mol} \mathrm{O}_{2} \mathrm{~m}^{-2} \mathrm{y}^{-1}$, with the largest source of error stemming from remineralization in the depths shallower than $200 \mathrm{~m}$ or greater than $800 \mathrm{~m}$, both of which are not well constrained by this technique. Net community production, as inferred from $\mathrm{O}_{2}$ and $\mathrm{Ar}$ seasonal cycles in the euphotic zone coupled to model simulations, is equal to of 3.0 to $5.2 \mathrm{~mol} \mathrm{O}_{2} \mathrm{~m}^{-2} \mathrm{y}^{-1}$, with the largest uncertainty stemming from the magnitude of winter-time production. Since the ${ }^{3} \mathrm{He}$ flux gauge is an estimate of how much nitrate is brought into the mixed layer through physical supply of upwelling water, it also gives an estimate of the $\mathrm{O}_{2}$ debt that would accompany such water. This large $\mathrm{O}_{2}$ debt contradicts the observed $\mathrm{O}_{2}$ maxima in the euphotic zone and several possible explanations are considered that could reflect on this paradox.

The spatial scales of these estimates range from regional scale for new production to local scale for net community production. Additionally, the export production estimate integrates over the longest period of time (years to decades), whereas the other estimates may be influenced by interannual variability. The net community production and export production estimates agree well with each other, in spite of this difference 
in temporal and spatial scales. In contrast the new production estimate is almost double the size of the other estimates. This discrepancy may reflect a significant degree of winter-time production that is not caught by the other estimates, the difference in temporal/spatial scales between three tracer methods, or uncertainties with the ${ }^{3} \mathrm{He}$ flux gauge technique. The largest uncertainty in the ${ }^{3} \mathrm{He}$ flux gauge technique is in the correlation between nitrate and ${ }^{3} \mathrm{He}$ since this correlation changes with time. Additionally, ${ }^{3} \mathrm{He}$ and $\mathrm{NO}_{3}^{-}$may have been decoupled in the more productive waters to the North, where high productivity between the base of the mixed layer and base of the euphotic zone could remove $\mathrm{NO}_{3}^{-}$while leaving ${ }^{3} \mathrm{He}$, resulting in the flux gauge yielding an overestimate of local production. Modeling studies could potentially assess the contribution of such lateral transport to the ${ }^{3} \mathrm{He}$ flux gauge estimate of new production.

The above conclusions are based on a fourteen month time-series that resolves the seasonal cycle but does not address interannual variability. Production stimulated by eddies may be a significant fraction of production at BATS (McGillicuddy et al., 2007). Two months in our time-series have been affected by eddies (as seen from temperature plots and satellite measurements). Samples from a full three year time-series have been collected. It will be interesting to measure the samples from the other years of the time-series and to see if the discrepancy between the production estimates persists. Additionally, the thermal and wind speed history differs from year to year and noble gas data from the other years will therefore give us a more robust parameterization of air-sea gas exchange.

Most of this thesis has been concerned with measurements in the upper few hundred meters of the ocean. Noble gases, however, may be valuable tracers in deep waters as well. Modeling studies suggest that noble gas concentrations in deep water may be diagnostic of diapycnal mixing (Henning et al., 2006; Ito and Deutsch, 2006) and gas dynamics during water mass formation (Hamme and Severinghaus, 2007). I have collected samples down to $4200 \mathrm{~m}$ on 12 occasions (once every 3 months) during the time-series. Only two such profiles have been analyzed so far. These profiles allow a glimpse of gas signatures in deep waters. Figure 6-1 presents the deep data from the November, 2004 profile of the noble gases. Helium and $\mathrm{Ne}$ increase slightly with depth, as does $\mathrm{SiO}_{2}$. Perhaps $\mathrm{He}$ and $\mathrm{Ne}$ are higher in waters from Antarctica because of higher winds and increased air injection there. Another possibility, however, is that the deeper samples have more bubbles entrained during sampling collection. When the deep samples were collected, water condensed on the outside of the tubing because of the low temperature of the samples, and thus it was 
harder to see whether bubbles were being entrained. Investigation of more deep profiles should lead to clues on whether this increased $\mathrm{He}$ and $\mathrm{Ne}$ signal is real.

The saturation anomaly of Ar reveals a "bulge" between $300 \mathrm{~m}$ and $1500 \mathrm{~m}$ that may be a result of diapycnal mixing and that qualitatively agrees remarkably well with the modeling results of Henning et al. (2006) for theoretical Ar distributions in the thermocline. Xenon is near equilibrium in the thermocline. The saturation anomaly of Xe decreases between 700 and $1600 \mathrm{~m}$, and then remains approximately constant for the remainder of the profile. Krypton has a similar saturation anomaly to Xe until about $700 \mathrm{~m}$, and then at greater depths is usually slightly more undersaturated than Xe. The gases may be undersaturated in deep water because of disequilibrium in the source regions (Hamme and Severinghaus, 2007). If the water in the source region cools rapidly, the heavier gases may not be able to equilibrate with the atmosphere before being subducted. However, one would then expect Xe to be more undersaturated than $\mathrm{Kr}$, since $\mathrm{Xe}$ has a stronger temperature dependence on solubility and a slower gas exchange time scale. The solubilities of the heavier noble gases, and in particular of Xe, are not well known, so perhaps the true Xe saturation anomaly is indeed more negative than $\mathrm{Kr}$. The other possibility is since diapycnal mixing would increase the saturation anomalies of the heavier gases whereas disequilibria during water mass formation would decrease the saturation anomalies, depending on the exact conditions and the temperature dependence on solubility, each gas balances these two effects in different ways. In any case, this profile is just one glance at deep water gas concentrations. Future work should include analyzing all 12 deep profiles that were obtained in this program and making quantitative arguments about the implications of the gas signatures.

In order to make full use of the deep profiles of $\mathrm{Xe}$ - and indeed to make better use of $\mathrm{Xe}$ in the shallow work as well - accurate determinations of the solubility of $\mathrm{Kr}$ and $\mathrm{Xe}$ in seawater are needed. The solubilities of $\mathrm{He}, \mathrm{Ne}, \mathrm{Ar}$, and $\mathrm{Kr}$ in seawater were determined by Weiss in the 1970s (Weiss, 1970, 1971; Weiss and Kyser, 1978) and the solubility of Xe was determined by Wood and Caputi (1966). Recent redeterminations of the solubilities of $\mathrm{Ne}$ and Ar by Hamme and Emerson (2004b) reveal up to 1\% differences from the Weiss solubilities. The solubilities of $\mathrm{Kr}$ and $\mathrm{Xe}$ have not yet been redetermined. Several lines of evidence suggest that the solubility of Xe may not be accurate. As described above, the deep profile shows Xe with deep saturations between $\mathrm{Ar}$ and $\mathrm{Kr}$, whereas one would expect the saturation anomalies of the gases to follow the order of Ar, then $\mathrm{Kr}$, and then Xe. One of the largest uncertainties in our determination of air-sea gas 
Figure 6-1: Saturation anomalies in deep water may yield information on diapycnal and ventilation characteristics. Saturation anomalies for (a) $\mathrm{He}, \mathrm{Ne}$ (b) $\mathrm{Ar}, \mathrm{Kr}$ and $\mathrm{Xe}$ in November, 2004. Dashed lines indicated a $0 \%$ saturation anomaly, i.e. the solubility equilibrium point. (c) For reference, profiles of temperature and nutrients on samples measured from the same CTD cast are shown as well. The circles represent data and the line represents a least squares smoothing spline that has been fit to the data.
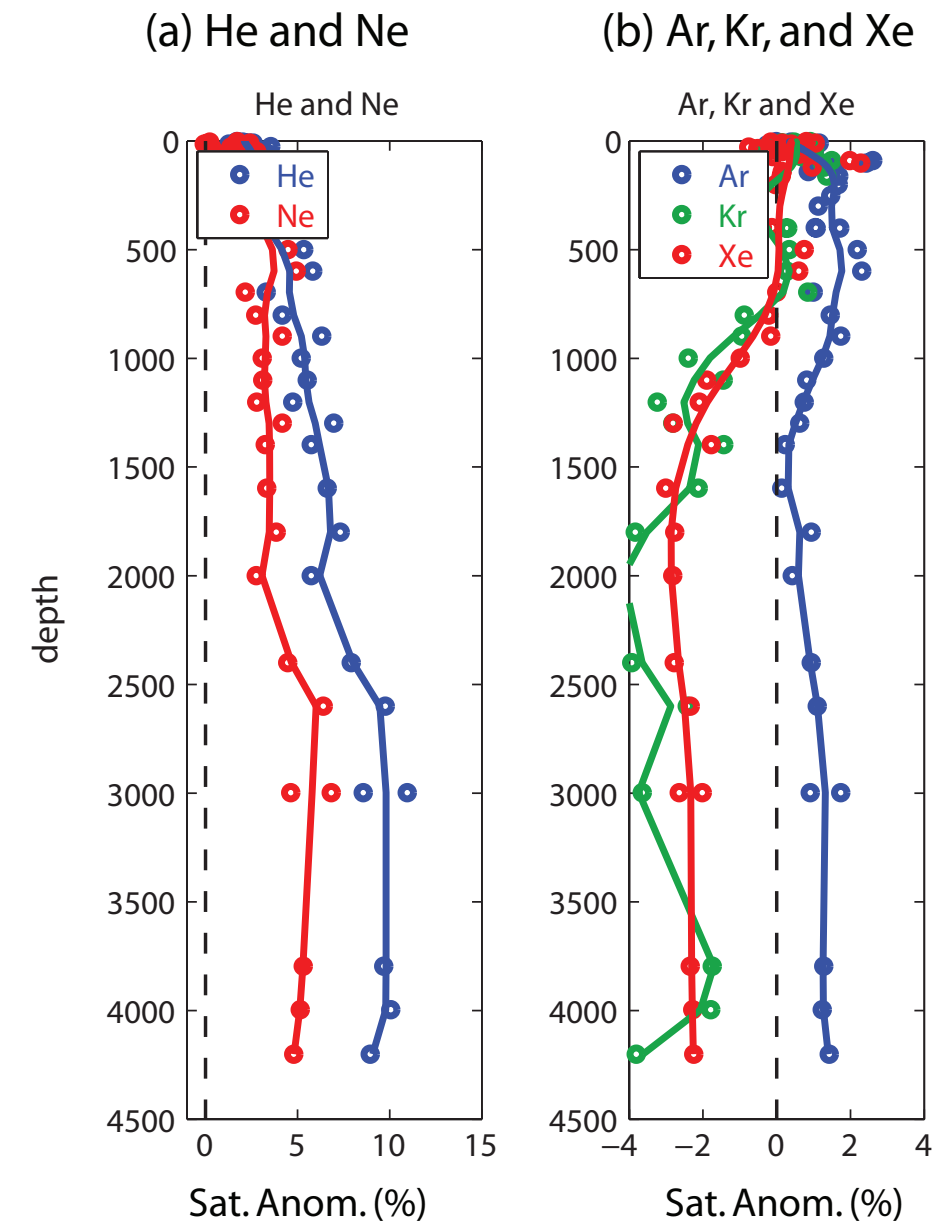

(c) Temp and Nutrients

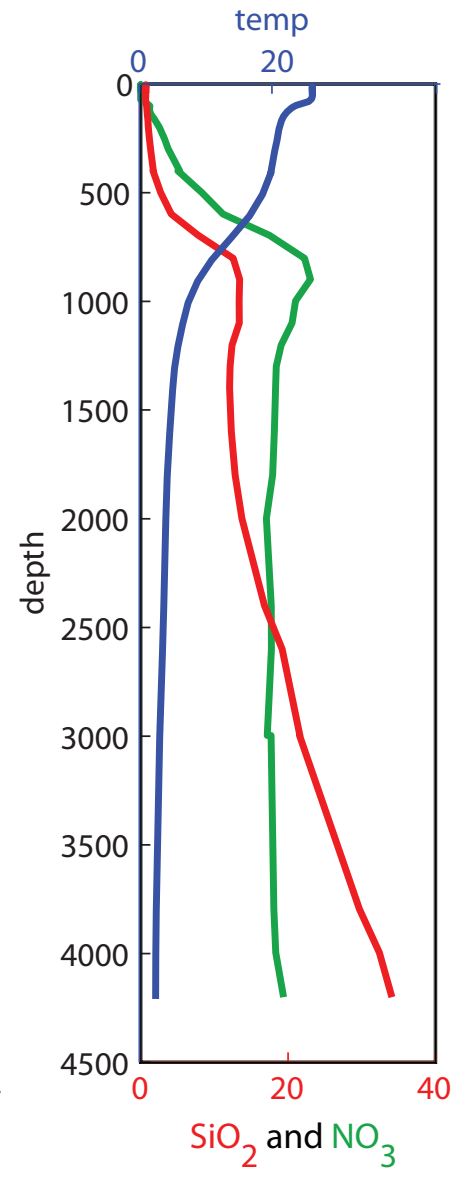


exchange parameters is the uncertainty in the Xe solubility. Now that it is clear that noble gases can be accurately measured in the ocean, the community needs accurate solubilities of all the noble gases in order to gain the most quantitative information from these tracers.

In summary, in this thesis a time-series of five noble gases is used to develop a parameterization of air-sea gas exchange that constrains diffusive gas exchange to $\pm 6 \%$ and air injection to $\pm 15 \%$. This parameterization can be used to calculate the total air-sea flux of any gas of interest in oligtrophic waters with wind speeds between 0 and $13 \mathrm{~m} \mathrm{~s}^{-1}$. Additionally, new, net community, and export production are quantified. The discrepency between the production estimates raises questions about exactly what these tracer techniques are measuring, about the timing of biological production and about nutrient cycling. 


\section{Bibliography}

W. Aeschbach-Hertig, F. Peeters, U. Beyerle, and R. Kipfer. Palaeotemperature reconstruction from noble gases in ground water taking into account equilibration with entrapped air. Nature, 405(6790):1040-1044, 2000 .

L.A. Anderson and J.L. Sarmiento. Redfield ratios of remineralization determined by nutrient data-analysis. Global Biogeochemical Cycles, 8(1):65-80, 1994.

T. R. Anderson and P. Pondaven. Non-redfield carbon and nitrogen cycling in the Sargasso Sea: pelagic imbalances and export flux. Deep-Sea Research Part I-Oceanographic Research Papers, 50(5):573-591, 2003.

W. E. Asher and R. Wanninkhof. The effect of bubble-mediated gas transfer on purposeful dual-gaseous tracer experiments. Journal of Geophysical Research-Oceans, 103(C5):10555-10560, 1998.

M. J. Behrenfeld, E. Boss, D. A. Siegel, and D. M. Shea. Carbon-based ocean productivity and phytoplankton physiology from space. Global Biogeochemical Cycles, 19(1), 2005.

M. L. Bender, D. T. Ho, M. B. Hendricks, R. Mika, M. O. Battle, P. P. Tans, T. J. Conway, B. Sturtevant, and N. Cassar. Atmospheric $\mathrm{O}_{2} / \mathrm{N}_{2}$ changes, 1993-2002: Implications for the partitioning of fossil fuel co2 sequestration. Global Biogeochemical Cycles, 19(4), 2005.

B.B. Benson and D. Jr. Krause. Isotopic fractionation of helium during solution: a probe for the liquid state. Journal of Solution Chemistry, 9:895-909, 1980.

U. Beyerle, W. Aeschbach-Hertig, M. Hofer, D. M. Imboden, H. Baur, and R. Kipfer. Infiltration of river water to a shallow aquifer investigated with ${ }^{3} \mathrm{H} /{ }^{3} \mathrm{He}$, noble gases and CFCs. Journal of Hydrology, 220 (3-4):169-185, 1999.

U. Beyerle, W. Aeschbach-Hertig, D. M. Imboden, H. Baur, T. Graf, and R. Kipfer. A mass spectrometric system for the analysis of noble gases and tritium from water samples. Environmental Science \& Technology, 34(10):2042-2050, 2000.

R.H. Bieri, M. Koide, and E.D. Goldberg. Geophysical implications of excess helium found in pacific waters. Journal of Geophysical Research, 72:2497-2511, 1967.

P.R. Bierman. Using in-situ produced cosmogenic ostopes to estimate rates of landscape evolution - a review from the geomorphic perspective. Journal of geophysical research - solid earth, 99:13885-13896, 1994.

W.S. Broecker and T.H. Peng. Gas-exchange rates between air and sea. Tellus, 26(1-2):21-35, 1974. 
K. O. Buesseler, C. H. Lamborg, P. W. Boyd, P. J. Lam, T. W. Trull, R. R. Bidigare, J. K. B. Bishop, K. L. Casciotti, F. Dehairs, M. Elskens, M. Honda, D. M. Karl, D. A. Siegel, M. W. Silver, D. K. Steinberg, J. Valdes, B. Van Mooy, and S. Wilson. Revisiting carbon flux through the ocean's twilight zone. Science, 316:567-570, 2007.

K.O. Buesseler. Do upper-ocean sediment traps provide an accurate record of particle flux? Nature, 353: 420-423, 1991.

K.O. Buesseler, A.F. Michaels, D.A Siegel, and A.H Knap. A three dimensional time-dependent approach to calibrating sediment trap fluxes. Global Biogeochemical Cycles, 12:297-310, 1994.

K.O. Buesseler, D.K Steinberg, A.F. Michaels, R.J. Johnson, J.E. Andrews, J.R. Valdes, and J.F. Price. A comparison of the quantity and quality of material caught in a neutrally buoyant versus a surface-tethered sediment trap. Deep-Sea Research I, 47:277-294, 2000.

J.H. Carpenter. The Chesapeake Bay Insitute technique for the Winkler oxygen method. Limnology and Oceanography, 10:141-143, 1965.

M. E. Carr, M. A. M. Friedrichs, M. Schmeltz, M. N. Aita, D. Antoine, K. R. Arrigo, I. Asanuma, O. Aumont, R. Barber, M. Behrenfeld, R. Bidigare, E. T. Buitenhuis, J. Campbell, A. Ciotti, H. Dierssen, M. Dowell, J. Dunne, W. Esaias, B. Gentili, W. Gregg, S. Groom, N. Hoepffner, J. Ishizaka, T. Kameda, C. Le Quere, S. Lohrenz, J. Marra, F. Melin, K. Moore, A. Morel, T. E. Reddy, J. Ryan, M. Scardi, T. Smyth, K. Turpie, G. Tilstone, K. Waters, and Y. Yamanaka. A comparison of global estimates of marine primary production from ocean color. Deep-Sea Research Part Ii-Topical Studies in Oceanography, 53(5-7):741-770, 2006.

R. J. Charlson, J. E. Lovelock, M. O. Andreae, and S. G. Warren. Oceanic phytoplankton, atmospheric sulfur, cloud albedo and climate. Nature, 326:665-661, 1987.

W. B. Clarke, W. J. Jenkins, and Z. Top. Determination of tritium by spectrometric measurement of ${ }^{3} \mathrm{He}$. International Journal of Applied Radiation and Isotopes, 27:515-522, 1976.

M.H. Conte, N. Ralph, and E.H. Ross. Seasonal and interannual variaibility in deep ocean particle fluxes at the Oceanic Flux Program (OFP)/BermudaAtlantic Time Series (BATS) site in the Western Sargasso Sea near Bermuda. Deep Sea Research II, 48:1471-1505, 2001.

P. M. Cox, R. A. Betts, C. D. Jones, S. A. Spall, and I. J. Totterdell. Acceleration of global warming due to carbon-cycle feedbacks in a coupled climate model. Nature, 408:1840187, 2000.

H. Craig and T. Hayward. Oxygen supersaturation in the ocean: biological versus physical contributions. Science, 235:199-202, 1987.

H. Craig and R. C. Wiens. Gravitational enrichment of Kr-84/Ar-36 ratios in polar ice caps: A measure of firn thickness and accumulation temperature. Science, 271(5256):1708-1710, 1996.

T. Dickey, D. Frye, H. Jannasch, E. Boyle, D. Manov, D. Sigurdson, J. McNeil, M. Stramska, A. Michaels, N. Nelson, D. Siegel, G. Chang, J. Wu, and A. Knap. Initial results from the Bermuda Testbed Mooring program. Deep-Sea Research Part I-Oceanographic Research Papers, 45(4-5):771-794, 1998. 
S.C. Doney. A synoptic atmospheric surface forcing data set and physical upper ocean model for the U.S. JGOFS Bermuda Atlantic Time-series Study Site. Journal of Geophysical Research, 101(C10):2561525634, 1996.

S.C. Doney and W.J. Jenkins. The effect of boundary conditions on tracer estimates of thermocline ventilation rates. Journal of Marine Research, 46:947-965, 1988.

R.C. Dugdale and J.J. Goering. Uptake of new and regenerated forms of nitrogen in primary productivity. Limnology and Oceanography, 12:196-206, 1967.

S. Emerson. Seasonal oxygen cycles and biological new production in surface waters of the Sub-Arctic Pacific ocean. Journal of Geophysical Research-Oceans, 92(C6):6535-6544, 1987.

S. Emerson, P. D. Quay, C. Stump, D. Wilbur, and R. Schudlich. Chemical tracers of productivity and respiration in the subtropical Pacific ocean. Journal of Geophysical Research-Oceans, 100(C8):1587315887, 1995.

R.W Eppley and B.J. Peterson. Particulate organic matter flux and planktonic new production in the deep ocean. Nature, 282(677-680):677-680, 1979.

L. S. Escandon, S. Ordonez, A. Vega, and F. V. Diez. Oxidation of methane over palladium catalysts: effect of the support. Chemosphere, 58(1):9-17, 2005.

C. W. Fairall, E. F Bradley, J. S. Godfrey, G. A. Wick, J. B. Edson, and G. S. Young. Cool-skin and warmlayer effects on sea surface temperature. Journal of Geophysical Research-Oceans, 101:1295-1308., 1996.

S. Fangohr and D. K. Woolf. Application of new parameterizations of gas transfer velocity and their impact on regional and global marine $\mathrm{CO}_{2}$ budgets. Journal of Marine Systems, 66:195-203, 2007.

K. A. Farley and E. Neroda. Noble gases in the earth's mantle. Annual Review of Earth and Planetary Sciences, 26:189-218, 1998.

C.B. Field, M.J. Behrenfeld, J.T. Randerston, and P. Falkowski. Primary production of the biosphere: Integrating terrestrial and oceanic components. Science, 281(5374):237-240, 1998.

J. Fluckiger, A. Dallenbach, T. Blunier, B. Stauffer, T. F. Stocker, D. Raynaud, and J.-M. Barnola. Variations in atmospheric $\mathrm{N}_{2} \mathrm{O}$ concentration during abrupt climatic changes. Science, 284:227-230, 1999.

N. M. Frew, E. J. Bock, U. Schimpf, T. Hara, H. Haussecker, J. B. Edson, W. R. McGillis, R. K. Nelson, S. P. McKenna, B. M. Uz, and B. Jahne. Air-sea gas transfer: Its dependence on wind stress, small-scale roughness, and surface films. Journal of Geophysical Research-Oceans, 109(C8), 2004.

N. M. Frew, D. M. Glover, E. J. Bock, and S. J. McCue. A new approach to estimation of global air-sea gas transfer velocty fields using dual-frequency altimeter backscatter. Journal of Geophysical Research, submitted, 2007.

G. Fuchs, W. Roether, and P. Schlosser. Excess 3He in the ocean surface layer. Journal of Geophysical Research, 92:6559-6568, 1987. 
W.D. Gardner. Sediment trap technology and sampling in surface waters. In R.B Hanson, H.W. Ducklow, and J.G. Field, editors, The Changing Ocean Carbon Cycle: midterm synthesis of the Joint Global Ocean Flux Study, pages 240-281. Cambridge University Press, 2000.

D. M. Glover, N. M. Frew, and S. J. McCue. Air-sea gas transfer velocity estimates from the Jason-1 and TOPEX altimeters: Prospects for a long-term global time series. Journal of Marine Systems, 66:173-181, 2007.

A. Graham, D. K. Woolf, and A. J. Hall. Aeration due to breaking waves. Part I: Bubble populations. Journal of Physical Oceanography, 34(5):989-1007, 2004.

N. Gruber and J.L. Sarmiento. Global patterns of marine nitrogen fixation and denitrification. Global Biogeochemical Cycles, 11(2):235-266, 1997.

N. Gruber, C.D. Keeling, and T.F. Stocker. Carbon-13 constraints on the seasonal inorganic carbon budget at the BATS site in the northwestern Sargasso Sea. Deep-Sea Research Part I, 45(4-5):673-717, 1998.

R. C. Hamme and S. R. Emerson. Measurement of dissolved neon by isotope dilution using a quadrupole mass spectrometer. Marine Chemistry, 91(1-4):53-64, 2004a.

R.C. Hamme and S. Emerson. The solubility of neon, nitrogen and argon in distilled water and seawater. Deep Sea Research I, 51(11):1517-1528, 2004b.

R.C. Hamme and S.R. Emerson. Constraining bubble dynamics and mixing with dissolved gases: Implications for productivity measurements by oxygen mass balance. Journal of Marine Research, 64:73-95, 2006.

R.C. Hamme and J.P Severinghaus. Dissolved inert gases as tracers of the imbalance between cooling and gas exchange during deep-water formation. Deep Sea Research, Part I, 54:939-950, 2007.

D. A. Hansell, N. R. Bates, and D. B. Olson. Excess nitrate and nitrogen fixation in the north atlantic ocean. Marine Chemistry, 84(3-4):243-265, 2004.

C. C. Henning, D. Archer, and I. Fung. Argon as a tracer of cross-isopycnal mixing in the thermocline. Journal of Physical Oceanography, 36(11):2090-2105, 2006.

P.J. Hernes, M.L. Peterson, J.W. Murray, S.G Wakeham, C. Lee, and J.I. Hedges. Particulate carbon and nitrogen fluxes and compositions in the central equatorial pacific. Deep-Sea Research I, 48:1999-2023, 2001.

H. Hiyagon, M. Ozima, B. Marty, S. Zashu, and H. Sakai. Noble-gases in submarine glasses from midoceanic ridges and Loihi seamount: Constraints on the early history of the earth. Geochimica Et Cosmochimica Acta, 56(3):1301-1316, 1992.

D. T. Ho, C. S. Law, M. J. Smith, P. Schlosser, M. Harvey, and P. Hill. Measurements of air-sea gas exchange at high wind speeds in the Southern Ocean: Implications for global parameterizations. Geophysical Research Letters, 33, 2006.

J.H. Hoffman and A.O. Nier. Atmospheric helium isotopic ratio. Geophysical Research Letters, 20:121-123, 1993. 
M. Honda, I. McDougall, D. B. Patterson, A. Doulgeris, and D. A. Clague. Possible solar noble-gas component in Hawaiian basalts. Nature, 349(6305):149-151, 1991.

E. M. Hood. Characterization of air-sea gas exchange processes and dissolved gas/ice interactions using noble gases. Ph.D. Thesis MIT/WHOI, WHOI 98-01, 1998.

T. Ito and C. Deutsch. Understanding the saturation state of argon in the thermocline: The role of air-sea gas exchange and diapycnal mixing. Global Biogeochemical Cycles, 20:doi:10.1029.2005GB002655, 2006.

B. Jähne, G. Heinz, and W. Dietrich. Measurement of the diffusion coefficients of sparingly soluble gases in water. Journal of Geophysical Research, 92(C10):10767-10776, 1987.

A. Janby, W. Clark, E. Noordally, S. Grimses, and S. Tahir. Noble metal catalysts for methane removal. Chemosphere, 52:1041-1046, 2003.

W. J. Jenkins. Tritium-helium dating in Sargasso Sea - measurement of oxygen utilization rates. Science, 196(4287):291-292, 1977. DA760 SCIENCE.

W. J. Jenkins. Tritium and He-3 in the Sargasso Sea. Journal of Marine Research, 38(3):533-569, 1980.

W. J. Jenkins. The use of anthropogenic tritium and He-3 to study sub-tropical gyre ventilation and circulation. Philosophical Transactions of the Royal Society of London Series a-Mathematical Physical and Engineering Sciences, 325:43-61, 1988a.

W. J. Jenkins. Nitrate flux into the euphotic zone near Bermuda. Nature, 331(6156):521-523, 1988b.

W. J. Jenkins and W. B. Clarke. The distribution of ${ }^{3} \mathrm{He}$ in the western Atlantic Ocean. Deep-Sea Research, 23:481, 1976.

W. J. Jenkins and S.C. Doney. The subtropical nutrient spiral. Global Biogeochemical Cycles, 17(4):1110, doi:10.1029/2003GB002085, 2003.

W. J. Jenkins and J. C. Goldman. Seasonal oxygen cycling and primary production in the Sargasso Sea. Journal of Marine Research, 43(2):465-491, 1985.

W.J. Jenkins. 3h and 3He in the Beta Triangle: Observations of gyre ventilation and oxygen utilization rates. Journal of Physical Oceanography, 17:763-783, 1987.

X. Jin, R.G. Najjar, F. Louanchi, and S.C Doney. A modeling study of the seasonal oxygen budget of the global ocean. Journal of Geophysical Research-Oceansm, 112:doi:10.1029/2006JC003731, 2007.

R. F. Keeling. On the role of large bubbles in air-sea gas-exchange and supersaturation in the ocean. Journal of Marine Research, 51(2):237-271, 1993.

R. F. Keeling, S. C. Piper, and M. Heimann. Global and hemispheric $\mathrm{CO}_{2}$ sinks deduced from changes in atmospheric $\mathrm{O}_{2}$ concentration. Nature, 381(6579):218-221, 1996.

R.F. Keeling, R. G. Najjar, M.L. Bender, and P.P. Tans. What atmospheric oxygen measurements tell us about the global carbon cycle. Global Biogeochemical Cycles, 7(1):37-67, 1993. 
R. Kistler, E. Kalnay, W. Collins, S. Saha, G. White, J. Woollen, M. Chelliah, W. Ebisuzaki, M. Kanamitsu, V. Kousky, H. van den Dool, R. Jenne, and M. Fiorino. The NCEP-NCAR 50-year reanalysis: Monthly means CD-ROM and documentation. Bulletin of the American Meteorological Society, 82(2):247-267, 2001.

A.J. Knap, A.F. Michaels, D.K. Steinberg, and et al. BATS methods manual, Version 4. US JGOFS Planning Office, Woods Hole, MA, 1997.

A. N. Knapp, D. M. Sigman, and F. Lipschultz. $\mathrm{N}$ isotopic composition of dissolved organic nitrogen and nitrate at the Bermuda Atlantic Time-series Study site. Global Biogeochemical Cycles, 19(1), 2005.

D. Krause and B.B Benson. The solubility and isotopic fractionation of gases in dilute aqueous-solution: 2a. Solubilities of the noble gases. Journal of Solution Chemistry, 18:823-872, 1989.

J. T. Kulongoski and D. R. Hilton. A quadrupole-based mass spectrometric system for the determination of noble gas abundances in fluids. Geochemistry Geophysics Geosystems, 3, 2002.

D. Lal. Cosmic-ray labeling of erosion surfaces - in situ nuclide production-rates and erosion models. Earth and Planetary Science Letters, 104:424-439, 1991.

E. A. Laws. Photosynthetic quotients, new production and net community production in the open ocean. Deep-Sea Research Part a-Oceanographic Research Papers, 38(1):143-167, 1991.

C. Le Quéré, C. Rodenbeck, E. T. Buitenhuis, T. J. Conway, R. Langenfelds, A. Gomez, C. Labuschagne, M. Ramonet, T. Nakazawa, N. Metzl, N. Gillett, and M. Heimann. Saturation of the Southern Ocean $\mathrm{CO}_{2}$ sink due to recent climate change. Science, 316:1735-1738, 2007.

V.G. Levich. Physiochemical Hydrodynamics. Prentice-Hall, Neward, N.J., 1962.

P.S. Liss and L. Merlivat. Air-sea gas exchange rates: Introduction and synthesis. In P. Buart-Menard, editor, The Role of Air-Sea Gas Exchange in Geochemical Cycling, pages 113-127. D. Reidel, Dordrecht, Holland, 1986.

D.E. Lott. Improvements in noble gas separation methodology: a nude cryogenic trap. Geochemistry, Geophysics, Geosystems, 2:10.129/2001GC000202, 2001.

D.E. Lott and W. J. Jenkins. Advances in analysis and shipboard processing of tritium and helium samples. International WOCE Newsletter, 30:27-30, 1998.

D.E. Lott. and W.J. Jenkins. An automated cryogenic charcoal trap system for helium isotope mass spectrometry. Review of Scientific Instruments, 55:1982-1988, 1984.

M. Lyubovsky and L. Pfefferle. Methane combustion over the alpha -alumina supported Pd catalyst: Activity of the mixed Pd/PdO state. Applied Catalysis A, 173:107-119, 1998.

D. MacMahon. Half-life evaluations for H-3, Sr-90, and Y-90. Applied Radiation and Isotopes, 64:14171419, 2006.

A. H. Manning and D. K. Solomon. Using noble gases to investigate mountain-front recharge. Journal of Hydrology, 275(3-4):194-207, 2003. 
J.H. Martin, G.A. Knauer, D.M. Karl, and W.W. Broenkow. VERTEX: carbon cycling in the northeast pacific. Deep-Sea Research, 34:267-285., 1987.

D. J. McGillicuddy, A. R. Robinson, D. A. Siegel, H. W. Jannasch, R. Johnson, T. Dickeys, J. McNeil, A. F. Michaels, and A. H. Knap. Influence of mesoscale eddies on new production in the Sargasso Sea. Nature, 394:263-268, 1998.

D. J. McGillicuddy, L. A. Anderson, N. R. Bates, T. Bibby, K. O. Buesseler, C. A. Carlson, C. S. Davis, C. Ewart, P. G. Falkowski, S. A. Goldthwait, D. A. Hansell, W. J. Jenkins, R. Johnson, V. K. Kosnyrev, J. R. Ledwell, Q. P. Li, D. A. Siegel, and D. K.j Steinbery. Eddy/wind interactions stimulate extraordinary mid-ocean plankton blooms. Science, 316:1021-1026, 2007.

D.J. McGillicuddy, R. Johnson, D. A. Siegel, A. F. Michael, N. R. Bates, and A. H. Knap. Mesoscale variations of biogeochemical properties in the Sargasso Sea. Journal of Geophysical Research-Oceans, 104:13381-13394, 1999.

G. A. McKinley, M. J. Follows, J. Marshall, and S. M. Fan. Interannual variability of air-sea $\mathrm{O}_{2}$ fluxes and the determination of $\mathrm{CO}_{2}$ sinks using atmospheric $\mathrm{O}_{2} / \mathrm{N}_{2}$. Geophysical Research Letters, 30(3), 2003.

L. Memery and L. Merlivat. Modelling the gas flux through bubbles at the air-water interface. Tellus, 37B: 272-285, 1985.

A.F. Michaels and A.H. Knap. Overview of the U.S. JGOFS Bermuda Atlantic Time-series Study and the Hydrostation S program. Deep-Sea Research II, 43:157-198, 1996.

A.F. Michaels, N.R Bates, K.O. Buesseler, C.A. Carlson, and A.H. Knap. Carbon system imbalances in the Sargasso Sea. Nature, 372:537-540, 1994.

E.C. Monahan and T. Torgersen. Enhancement of air-sea gas exchange by oceanic whitecapping. In AirWater Mass Transfer, Second International Symposium on Gas Transfer at Water Surfaces, pages 608617. 1990.

B. Mourino-Carballido and D. J. McGillicuddy. Mesoscale variability in the metabolic balance of the Sargasso Sea. Limnology and Oceanography, 51:2675-2689, 2006.

J.W. Murray, J. Young, J. Newton, J. Dunne, R. Chapin, and B. Paul. Export flux of particulate organic carbon from the central equatorial pacific determined using a combined drifting trap- 234Th approach. Deep-Sea Research II, 43:1093-1132, 1996.

D. L. Musgrave, J. Chou, and W. J. Jenkins. Application of a model of upper-ocean physics for studying seasonal cycles of oxygen. Journal of Geophysical Research-Oceans, 93:15679-15700, 1988.

R. G. Najjar and R. F. Keeling. Analysis of the mean annual cycle of the dissolved oxygen anomaly in the world ocean. Journal of Marine Research, 55:117-151, 1997.

P.D. Nightingale, G. Malin, C.S. Law, A. Watson, P.S. Liss, M.I. Liddicoat, J. Boutin, and R. UpstillGoddard. In situ evaluation of air-sea gas exchange parameterizations using novel conservative and volatile tracers. Global Biogeochemical Cycles, 14(1):373-387, 2000. 
S. Ono, A. Ennyu, R. G. Najjar, and N. R. Bates. Shallow remineralization in the Sargasso Sea estimated from seasonal variations in oxygen, dissolved inorganic carbon and nitrate. Deep-Sea Research, 48: 1567-1582, 2001.

K.M. Orcutt, F. Lipschultz, Gunderson K., Arimoto R., A.F. Michaels, A.H Knap, and J.R. Gallon. A seasonal study of the significance of $\mathrm{N}_{2}$ fixation by Trichodesmium spp. at the Bermuda Atlantic Timeseries Study (BATS) site. Deep Sea Research II, 48:1583-1608, 2001.

T.H. Peng, W.S. Broecker, G.G. Mathieu, Y.H. Li, and A.E. Bainbridge. Radon evasion rates in the Atlantic and Pacific oceans as determined during the GEOSECS program. Journal of Geophysical Research, 84: 2471-2486, 1979.

J. R. Petit, J. Jouzel, D. Raynaud, N. I. Barkov, J. M. Barnola, I. Basile, M. Bender, J. Chappellaz, M. Davis, G. Delaygue, M. Delmotte, V. M. Kotlyakov, M. Legrand, V. Y. Lipenkov, C. Lorius, L. Pepin, C. Ritz, E. Saltzman, and M. Stievenard. Climate and atmospheric history of the past 420,000 years from the Vostok ice core, Antarctica. Nature, 399(6735):429-436, 1999.

J. C. Poole, G. W. McNeill, S. R. Langman, and F. Dennis. Analysis of noble gases in water using a quadrupole mass spectrometer in static mode. Applied Geochemistry, 12(6):707-714, 1997.

J.F. Price, R.A. Weller, and R. Pinkel. Diurnal cycling: observations and models of the upper ocean response to diurnal heating, cooling, and wind mixing. Journal of Geophysical Research-Oceans, 91(C7):84118427, 1986.

G.A. Riley. Oxygen, phosphate and nitrate in the Atlantic Ocean. Bulletin of the Bingham Oceanographic Collection, 13:1-126, 1951.

R. Sambrotto, G. Savidge, C. Robinson, P. Boyd, T. Takahashi, D. M. Karl, C. Langdon, D. Chipman, J. Marra, and L. Codispoti. Elevated consumption of carbon relative to nitrogen in the surface ocean. Nature, 363:248-250, 1993.

Y. Sano and N. Takahata. Measurement of noble gas solubility in seawater using a quadrupole mass spectrometer. Journal of Oceanography, 61(3):465-473, 2005.

J.L. Sarmiento, G. Thiele, R.M. Key, and W.S. Moore. Oxygen and nitrate new production and remineralization in the North-Atlantic subtropical gyre. Journal of Geophysical Research-Oceans, 95:18303-18315, 1990 .

J.M. Schafer, S. Ivy-Ochs, R. Wieler, J. Leya, H. Baur, G.H. Denton, and C. Schluchter. Cosmogenic noble gas studies in the oldest landscape on earth: surface exposure ages of the dry valleys, antarctica. Earth and Planetary Science Letters, 167:215-226, 1999.

E. Schroeder and H. Stommel. How representative is the series of PANULIRUS stations of monthly mean conditions off Bermuda? Progress in Oceanography, 5:31-40, 1969.

J. P. Severinghaus, A. Grachev, and M. Battle. Thermal fractionation of air in polar firn by seasonal temperature gradients. Geochemistry Geophysics Geosystems, 2:art. no.-2000GC000146, 2001.

J. P. Severinghaus, A. Grachev, B. Luz, and N. Caillon. A method for precise measurement of argon 40/36 and krypton/argon ratios in trapped air in polar ice with applications to past firn thickness and abrupt 
climate change in Greenland and at Siple Dome, Antarctica. Geochimica Et Cosmochimica Acta, 67(3): 325-343, 2003.

U. Siegenthaler and J. L. Sarmiento. Atmospheric carbon-dioxide and the ocean. Nature, 365:119-125, 1993.

R. Spahni, J. Chappelax, T. F. Stocker, L. Loulergue, G. Hausammann, K. Kawamura, J. Fluckiger, J. Schwander, D. Raynaud, V. Masson-Delmotte, and J. Jouzel. Atmospheric methane and nitrous oxide of the late pleistocene from antarctic ice cores. Science, 310:1317-1321, 2005.

W. S. Spitzer. Rates of vertical mixing, gas exchange and net production: Estimates from seasonal gas cycles in the upper ocean near Bermuda. Ph.D. Thesis MIT/WHOI, WHOI-89-30, 1989.

W. S. Spitzer and W. J. Jenkins. Rates of vertical mixing, gas-exchange and new production - estimates from seasonal gas cycles in the upper ocean near Bermuda. Journal of Marine Research, 47:169-196, 1989.

R. H. R. Stanley, K. O. Buesseler, S. J. Manganini, D. K. Steinberg, and J. R. Valdes. A comparison of major and minor elemental fluxes collected in neutrally buoyant and surface-tethered sediment traps. Deep-Sea Research Part I-Oceanographic Research Papers, 51(10):1387-1395, 2004.

R. H. R. Stanley, W. J. Jenkins, and S. C. Doney. Quantifying seasonal air-sea gas exchange processes using noble gas time-series: A design experiment. Journal of Marine Research, 64(2):267-295, 2006.

D.K. Steinberg, C.A. Carlson, N.R. Bates, S.A. Goldthwait, L.P. Madin, and A.F. Michaels. Zooplankton vertical migration and the active transport of dissolved organic and inorganic carbon in the Sargasso Sea. Deep-Sea Research I, 47:137-158, 2000.

H. Stommel. Determination of water mass properties of water pumped down from the Ekman layer to the geostrophic flow below. Proceedings of the National Academy of Science, U.S.A., 36:3051-3055, 1979.

M. Stute, C. Sonntag, J. Deak, and P. Schlosser. Helium in deep circulating groundwater in the great hungarian plain: Flow dynamics and crustal and mantle helium fluxes. Geochimica Et Cosmochimica Acta, 56(5):2051-2067, 1992.

M. Stute, J. F. Clark, P. Schlosser, W. S. Broecker, and G. Bonani. A 30,000-yr continental paleotemperature record derived from noble-gases dissolved in groundwater from the San Juan basin, New Mexico. Quaternary Research, 43(2):209-220, 1995.

C. Sweeney, E. Gloor, A. R. Jacobson, R. M. Key, G. McKinley, J. L. Sarmineto, and R. Wanninkhof. Constraining global air-sea gas exchange for $\mathrm{CO}_{2}$ with recent bomb ${ }^{14} \mathrm{C}$ measurements. Global Biogeochemical Cycles, 21:do1:10.1029/2006GB002784, 2007.

E. N. Sweeney, D. J. McGillicuddy, and K. O Buesseler. Biogeochemical impacts due to mesoscale eddy activity in the Sargasso Sea as measured at the Bermuda Atlantic Time-series Study (BATS). Deep-Sea Research Part Ii-Topical Studies in Oceanography, 50:3017-3039., 2003.

T. Takahashi, R. A. Feely, R. F. Weiss, R. H. Wanninkhof, D. W. Chipman, S. C. Sutherland, and T. T. Takahashi. Global air-sea flux of $\mathrm{CO}_{2}$ : An estimate based on measurements of sea-air $\mathrm{pCO}_{2}$ difference. Proceedings of the National Academy of Sciences of the United States of America, 94:8292-8299, 1997. 
J.R. Valdes and J.F. Price. A neutrally buoyant, upper ocean sediment trap. Journal of Atmospheric and Oceanographic Technology, 17:62-68, 2000.

R. Wanninkhof. Relationship between wind speed and gas exchange over the ocean. Journal of Geophysical Research, 97(C5):7373-7382, 1992.

R. Wanninkhof and W.R. McGillis. A cubic relationship between air-sea $\mathrm{CO}_{2}$ exchange and wind speed. Geophysical Research Letters, 26(13):1889-1892, 1999.

A.J. Watson, R.C. Upstill-Goddard, and P.S. Liss. Air-sea gas exchange in rough and stormy seas measured by a dual-tracer technique. Nature, 349:145-147, 1991.

D. W. Waugh, T. M. Hall, and T. W. N. Haine. Relationships among tracer ages. Journal of Geophysical Research-Oceans, 108(C5):art. no.-3138, 2003.

R.F. Weiss. The solubility of nitrogen, oxygen and argon in water and seawater. Deep-Sea Research, 17: 721-735, 1970.

R.F. Weiss. Solubility of helium and neon in water and seawater. Journal of Chemical Engineering Data, 16:235-241, 1971.

R.F. Weiss and T.K. Kyser. Solubility of krypton in water and seawater. Journal of Chemical Engineering Data, 23(1):69-72, 1978.

G. A. Wick, J. C. Ohlmann, C. W. Fairall, and A. T. Jessup. Improved oceanic cool-skin corrections using a refined solar penetration model. Journal of Physical Oceanography, 35:1986-1996, 2005.

R. G. Williams and M. J. Follows. The Ekman transfer of nutrients and maintenance of new production over the north atlantic. Deep-Sea Research Part I-Oceanographic Research Papers, 45(2-3):461-489, 1998.

R.W. Williams, M.A. Spall, and J.C. Marshall. Does Stommel's mixed layer "demon" work? Journal of Physical Oceanography, 25:3089-31-2, 1995.

D. Wood and R. Caputi. Solubilities of $\mathrm{Kr}$ and Xe in fresh and sea water. Technical Report Technical Report USNRDL-TR-988, U.S. Naval Radiological Defense Laboratory, 1966.

D. K. Woolf. Bubbles and the air-sea transfer velocity of gases. Atmosphere-Ocean, 31(4):517-540, 1993.

D. K. Woolf and S. A. Thorpe. Bubbles and the air-sea exchange of gases in near-saturation conditions. Journal of Marine Research, 49(3):435-466, 1991.

D. K. Woolf, I. S. Leiferb, P.D. Nightingale, T. S. Rheed, P. Bowyere, G. Caluuiez, G. de Leeuw, S. E. Larsen, M. Luddicat, J. Baker, and M. O. Andrae. Modelling of bubble-mediated gas transfer: Fundamental principles and a laboratory test. Journal of Marine Systemsk, 66:71-91, 2007.

D.K Woolf. Parameterization of gas transfer velocities and sea-state-dependent wave breaking. Tellus Series B-Chemical and Physical Meteorology, 57:87-94, 2005.

L.V. Worthington. On the North Atlantic circulation, volume 6 of The Johns Hopkins Oceanographic Studies. Johns Hopkins University Press, Baltimore, 1976. 
J. Wu, W. Sunda, E. A. Boyle, and D. Karl. Phosphate depletion in the Western North Atlantic ocean. Science, 289:759-762, 2000.

W.Q. Zhang, W. Perrie, and S. Vagle. Impacts of winter storms on air-sea gas exchange. Geophysical Research Letters, 33, 2006.

X. Zhang and W. J. Cai. On some biases of estimating the global distribution of air-sea $\mathrm{CO}_{2}$ flux by bulk parameterizations. Geophysical Research Letters, 34:doi:10.1029/2006GL027337, 2007.

Z. Zhou, C. J. Ballentine, R. Kipfer, M. Schoell, and S. Thibodeaux. Noble gas tracing of groundwater/coalbed methane interaction in the San Juan Basin, USA. Geochimica Et Cosmochimica Acta, 69(23): 5413-5428, 2005. 